\title{
The Structural Origins of Intense Circular Dichroism in a Waggling Helicene Nanoribbon
}

Nathaniel J. Schuster, ${ }^{* 1}$ Leo A. Joyce, ${ }^{3}$ Daniel W. Paley, ${ }^{2}$ Fay Ng, ${ }^{1}$ Michael L. Steigerwald, ${ }^{* 1}$ and Colin Nuckolls*1

1Department of Chemistry and 2Columbia Nano Initiative, Columbia University, New York, New York 10027, United States

${ }^{3}$ Department of Process Research and Development, Merck and Co., Inc., Rahway, New Jersey 07065, United States

Email addresses of the corresponding authors: njs2154@columbia.edu, mls2064@columbia.edu, and cn37@columbia.edu

\section{Table of Contents}

I. Figures Referenced in the Article S2

$\begin{array}{ll}\text { II. General Experimental Information } & \text { S8 }\end{array}$

III. Synthetic Procedures and Characterization Data S12

IV. ${ }^{1} \mathrm{H}-\mathrm{NMR}$ and ${ }^{13} \mathrm{C}-\mathrm{NMR}$ Spectra $\quad \mathbf{S 1 8}$

V. DFT Calculations: Structures and Thermochemistry S23

a. $\mathrm{hPDl}_{2} \quad \mathrm{~S} 23$

b. $\mathrm{WH}[6] \quad \mathrm{S} 26$

c. $\mathrm{WH}[6][6] \quad \mathrm{S} 46$

VI. TDDFT-Calculated Spectra and Excited State Data S66

a. $\mathrm{WH}[6] \quad \mathrm{S66}$

b. $\mathrm{WH}[6][6] \quad \mathrm{S} 75$

VII. Beer-Lambert Plots of WH[6] and WH[6][6] S84

VIII. Single-Crystal X-Ray Diffraction Data of WH[6] S85

IX. ECD Spectra of NPDH, NP3H, WH[6], and WH[6][6] S87

$\begin{array}{lll}X . & \text { References } & \mathbf{S 8 8}\end{array}$ 


\section{Figures Referenced in the Article}
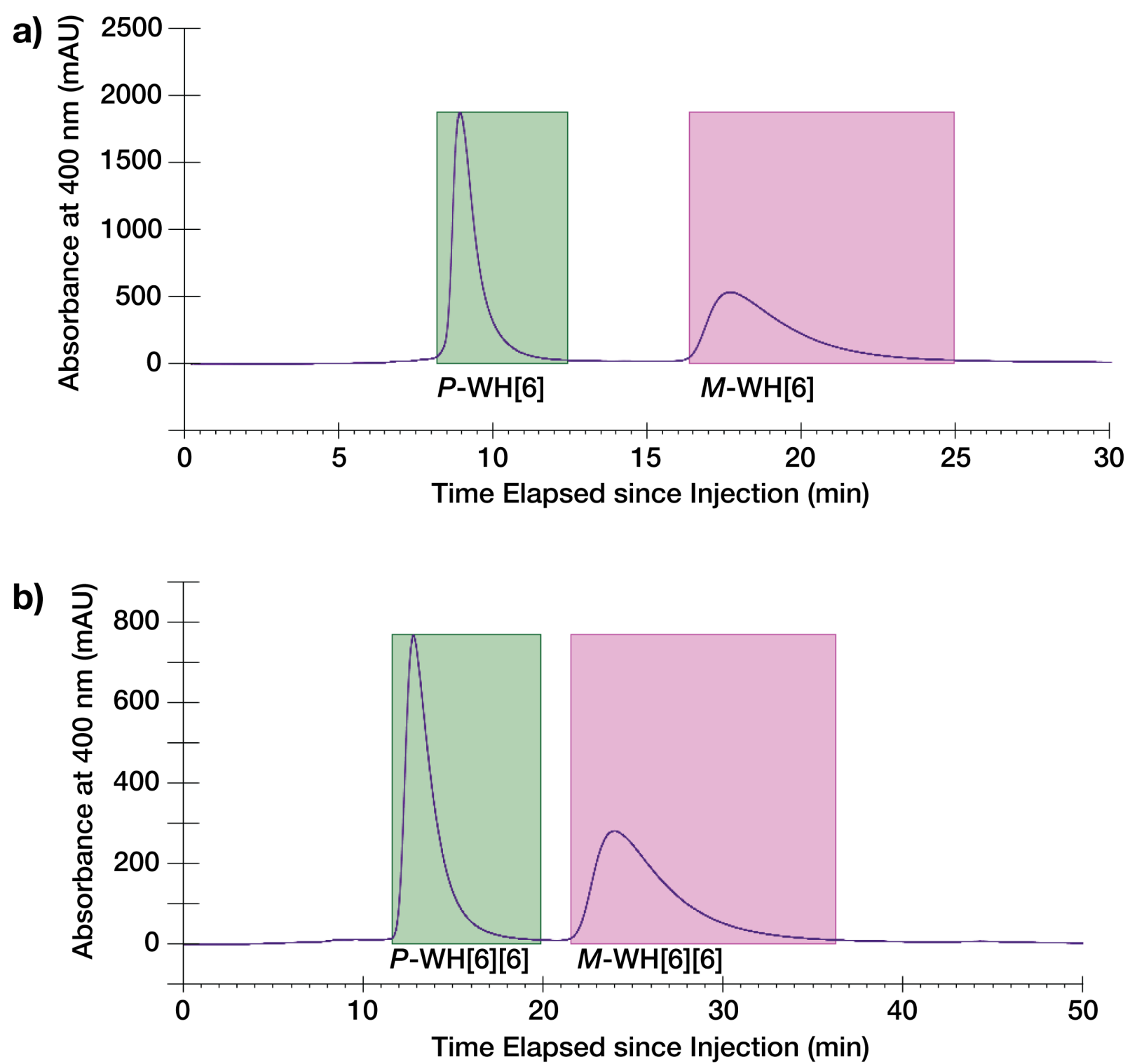

Figure S1. Resolution of the enantiomers of WH[6] (a) and WH[6][6] (b) by chiral preparative HPLC. The global chirality of the enantiomers (i.e., $M$ or $P$ ) was assigned by comparing the ECD spectra of these resolved species to the TDDFT-predicted ECD spectra of $\mathbf{W H}[6]$ and $\mathbf{W H}[6][6]$. The enantiomers of WH[6] were resolved from $25 \mathrm{mg}$ of racemic mixture dissolved in $10 \mathrm{~mL}$ of $20 \% \mathrm{DCM} /$ hexanes. This solution was injected in $\leq 4 \mathrm{~mL}$ aliquots onto a CHIRALPAK ${ }^{\circledR}$ IA column $(21 \mathrm{~mm}$ I.D. $\times 250 \mathrm{~mm}, 5 \mu \mathrm{m})$, with $32 \% \mathrm{DCM} /$ hexanes eluent flowing at $20 \mathrm{~mL} \mathrm{~min}^{-1}$ at room temperature. The enantiomers of WH[6][6] were resolved from $10 \mathrm{mg}$ of racemic mixture dissolved in $10 \mathrm{~mL}$ of $30 \% \mathrm{DCM} / \mathrm{hexanes}$. This solution was injected in $\leq 5 \mathrm{~mL}$ aliquots onto a CHIRALPAK ${ }^{\circledR}$ IA column $(21 \mathrm{~mm}$ I.D. $\times 250 \mathrm{~mm}, 5 \mu \mathrm{m})$, with $30 \%$ $\mathrm{DCM} /$ hexanes eluent flowing at $20 \mathrm{~mL} \mathrm{~min}^{-1}$ at room temperature. Each shaded box designates the fraction of each peak that was collected. 

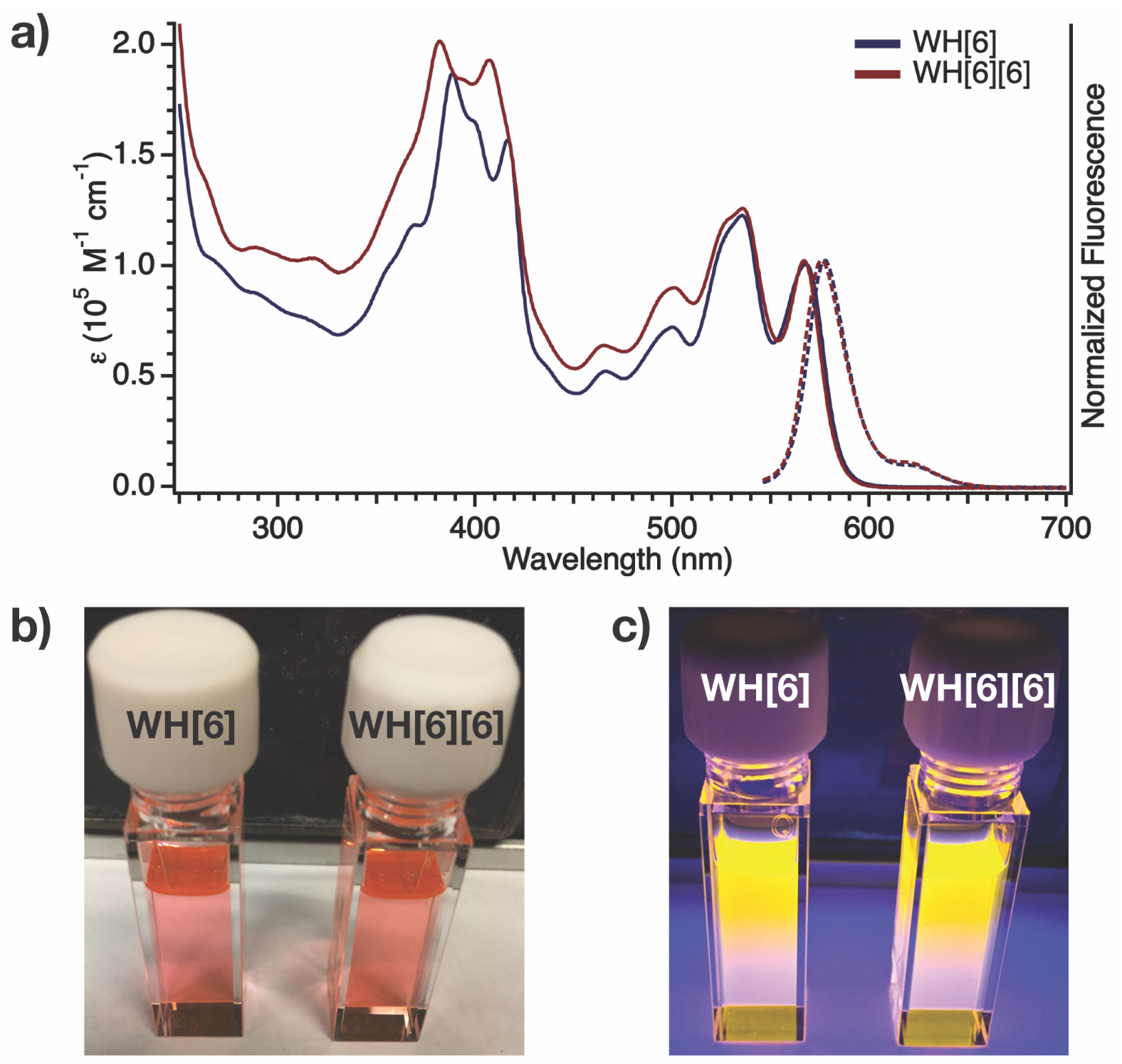

Figure S2. (a) UV-visible absorbance and fluorescence spectra of racemic WH[6] $\left(10^{-6} \mathrm{M}, \lambda_{\mathrm{ex}}=536 \mathrm{~nm}\right)$ and racemic $W H[6][6] ~\left(10^{-6} \mathrm{M}, \lambda_{\mathrm{ex}}=536 \mathrm{~nm}\right)$ in dichloromethane $(1 \mathrm{~cm}$ path length) at room temperature. (b) Solutions of WH[6] and WH[6][6] in dichloromethane (10-6 M) under ambient light, and (c) the same solutions when irradiated with long-wave UV light $(\sim 365 \mathrm{~nm})$ in the dark. 

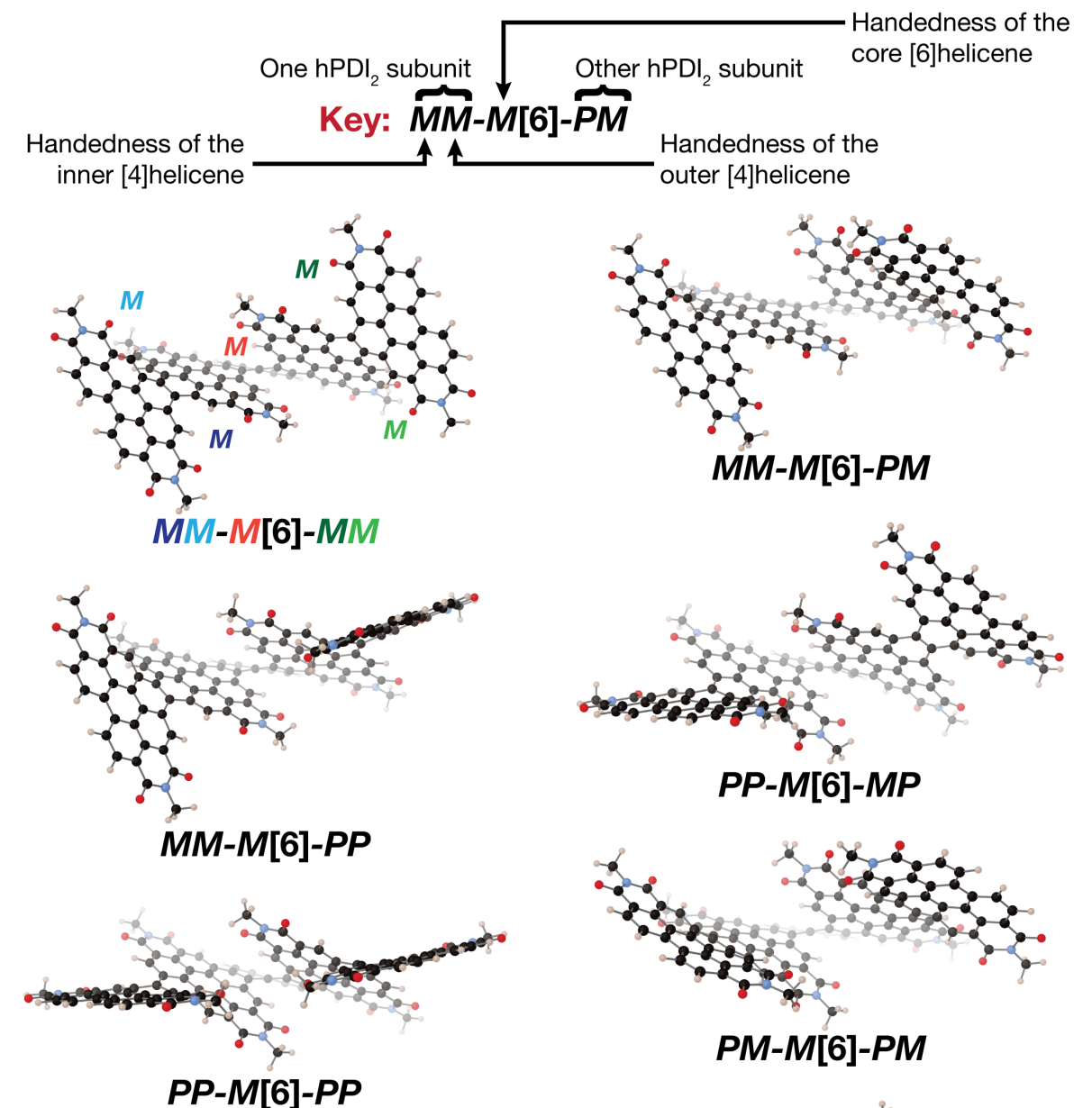

PM-M[6]-PM
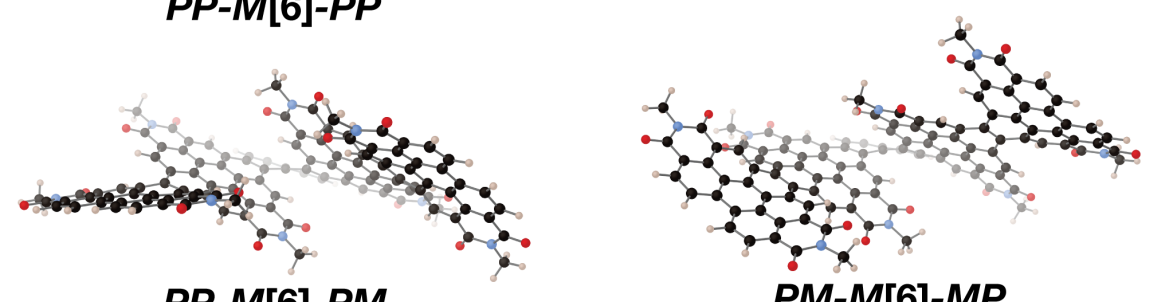

PM-M[6]-MP

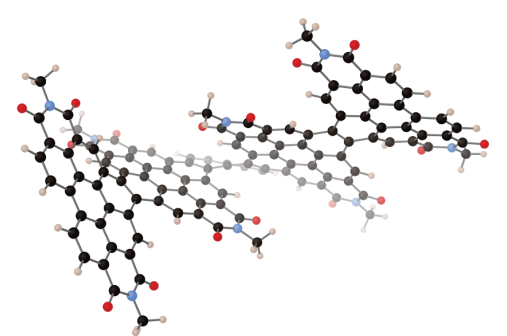

MM-M[6]-MP

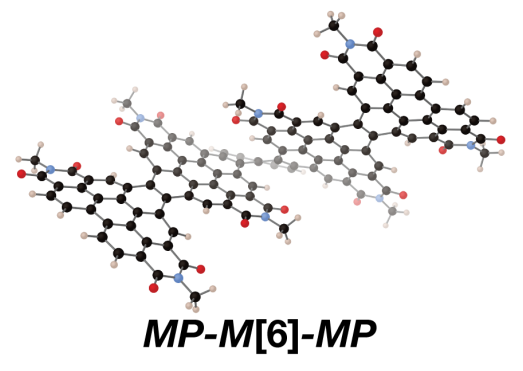

Figure S3. The ten left-handed conformational isomers of WH[6] that arise by altering the handedness of the [4]helicene subunits (i.e., $\pi$-conformations). Each isomer was optimized in the ground state and in the gas phase at the B3LYP/6-31G** level of DFT. Methyl groups substitute for the $\mathrm{CH}\left(\mathrm{C}_{5} \mathrm{H}_{11}\right)_{2}$ tails to simplify the calculations. The isomer nomenclature is described at the top of the page. For the name of a given isomer, exchanging one $\mathrm{hPDI}_{2}$ subunit for the other does not change the identity of the isomer (e.g., MM-M[6]-PP = PP-M[6]-MM); thus, two names may be used to identify the same isomer. 

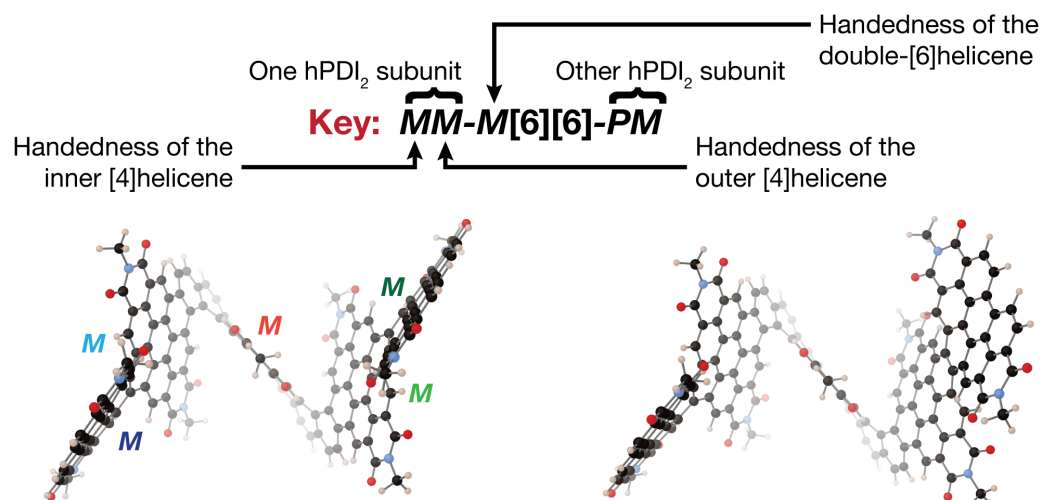

MM-M[6][6]-MM
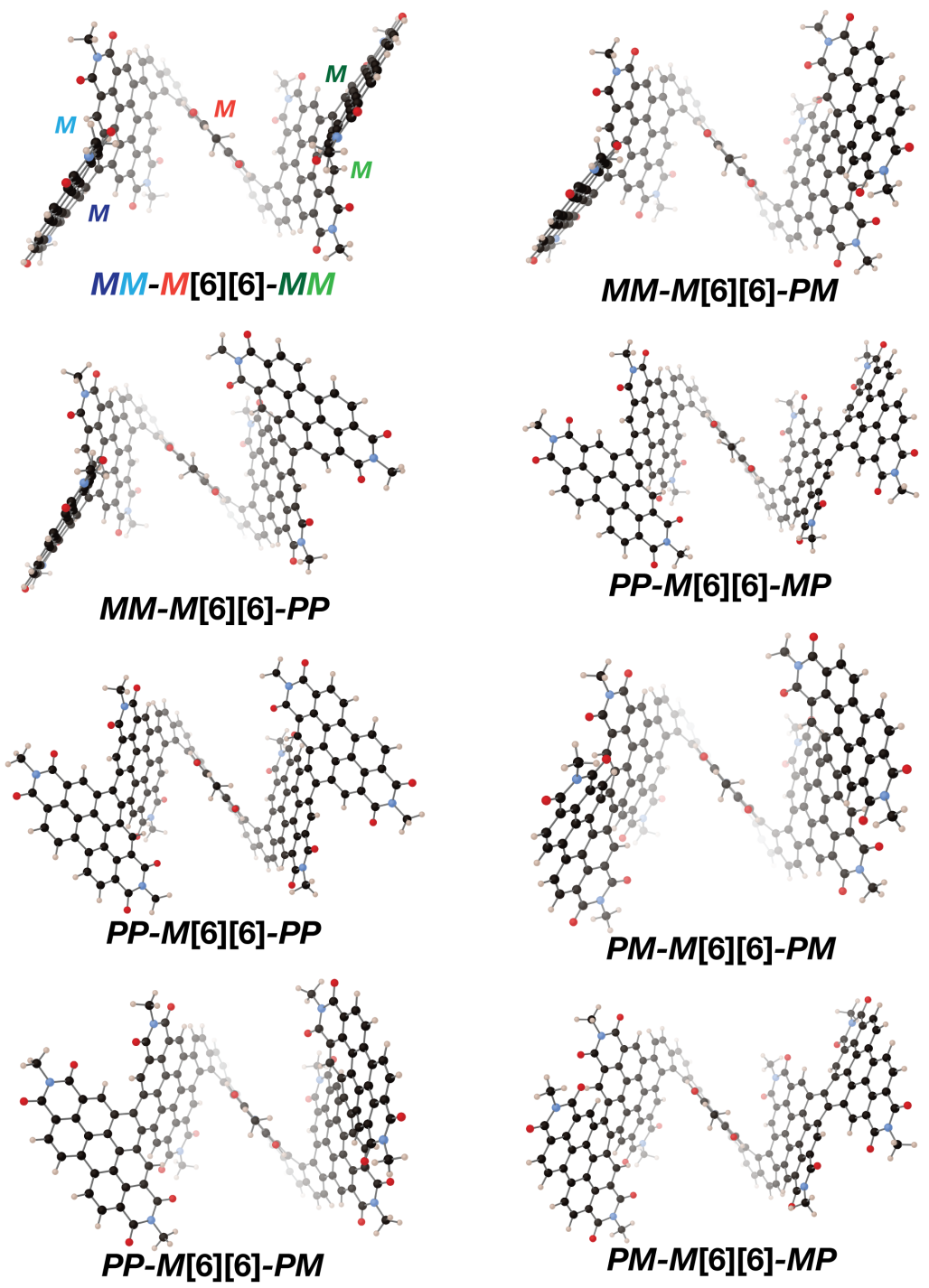

$P M-M[6][6]-P M$

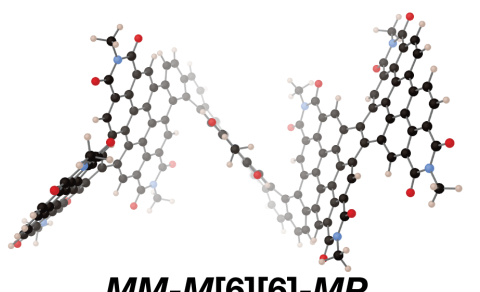

$M M-M[6][6]-M P$

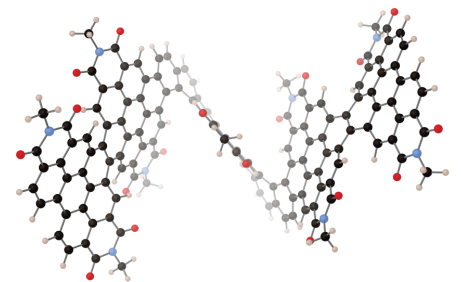

$P M-M[6][6]-M P$

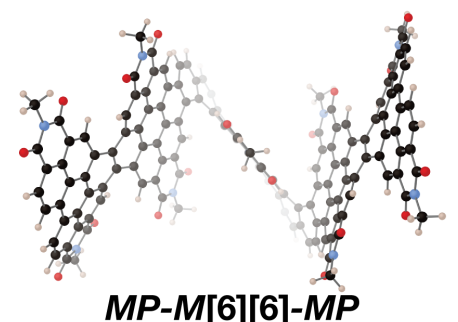

Figure S4. The ten left-handed conformational isomers of WH[6][6] that arise by altering the handedness of the [4]helicene subunits (i.e., $\pi$-conformations). Each isomer was optimized in the ground state and in the gas phase at the B3LYP/6-31G** level of DFT. Methyl groups substitute for the $\mathrm{CH}\left(\mathrm{C}_{5} \mathrm{H}_{11}\right)_{2}$ tails to simplify the calculations. The isomer nomenclature is described at the top of the page. For the name of a given isomer, exchanging one $\mathrm{hPDI}_{2}$ subunit for the other does not change the identity of the isomer (e.g., MM-M[6][6]-PP = PP-M[6][6]-MM); thus, two names may be used to identify the same isomer. 

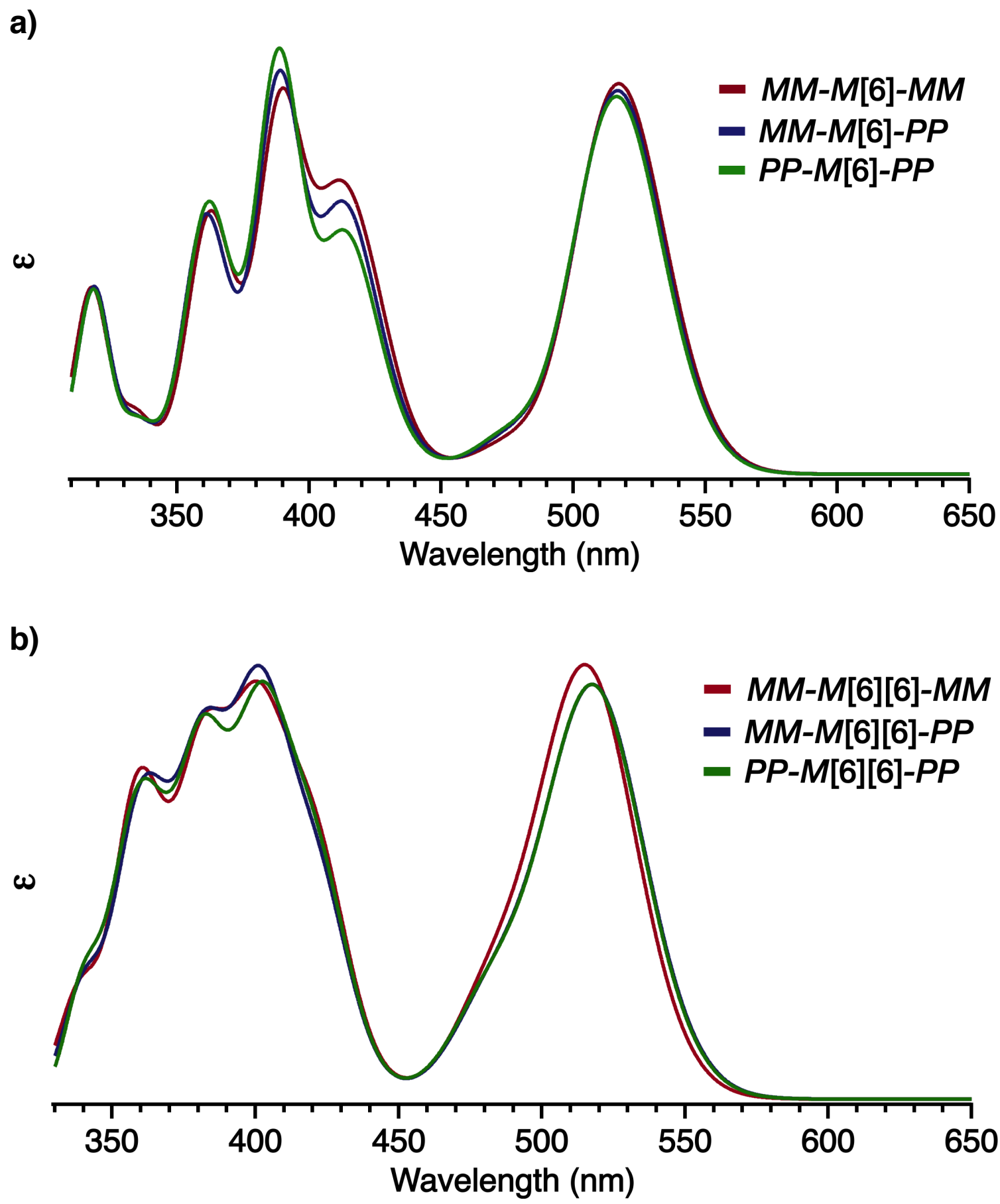

Figure S5. TDDFT-predicted (CAM-B3LYP/6-31++G**) UV-visible absorbance spectra of the three dominant left-handed conformations of WH[6] (a) and WH[6][6] (b). The absorbance intensities have not been normalized; instead, the relative molar absorptivities calculated for these conformations are depicted. Details concerning the TDDFT calculations and the plotting of these spectra can be found in Sections II and VI. 
a)

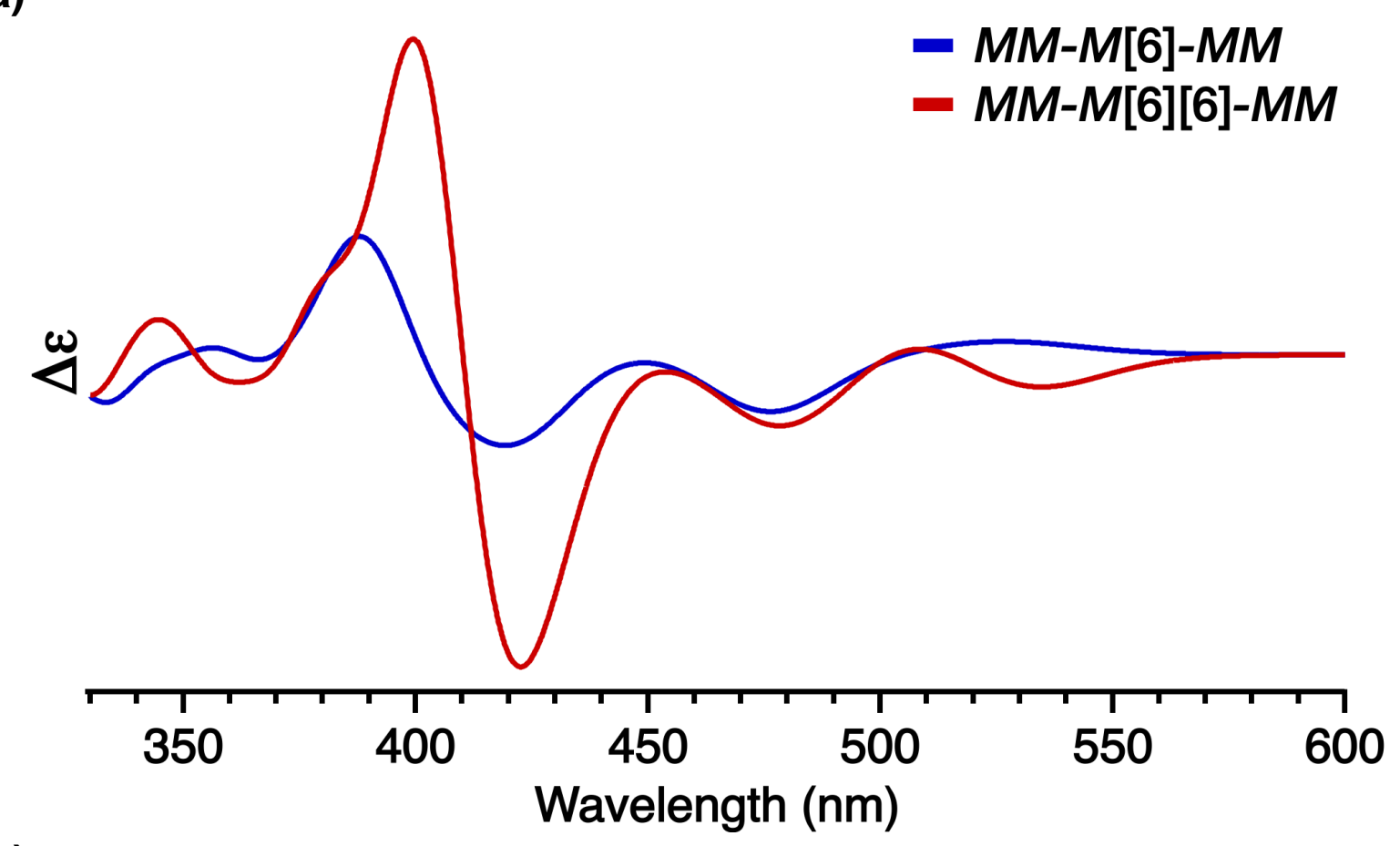

b)

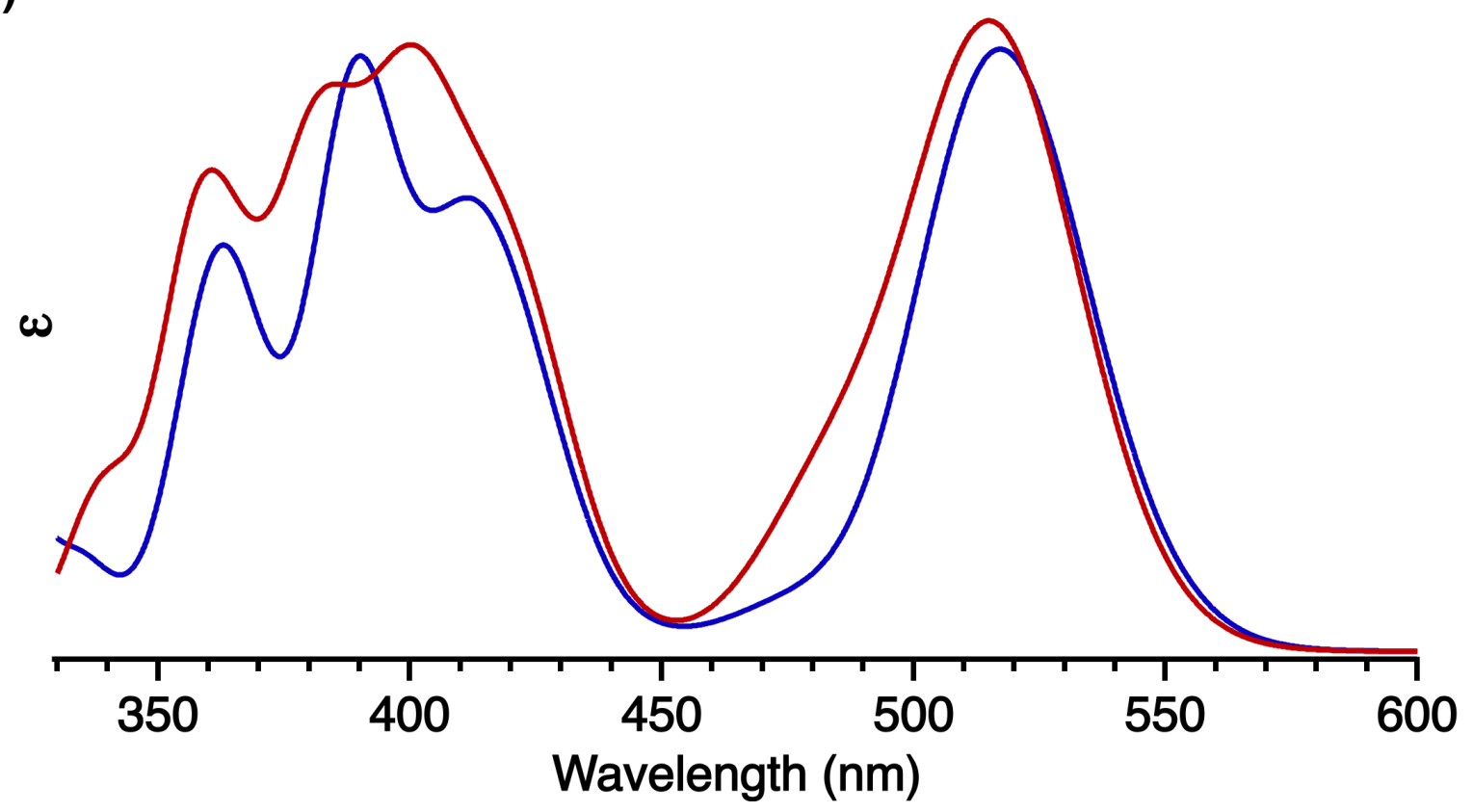

Figure S6. TDDFT-predicted (CAM-B3LYP/6-31++ $\mathrm{G}^{* *}$ ) ECD spectra (a) and UV-visible absorbance spectra (b) of $M M-M[6]-M M$ and $M$ M-M[6][6]-MM. The intensities of their ECD and UV-visible absorbances have not been normalized; instead, the relative molar ECD and molar absorptivities predicted for these species are depicted. Notably, the calculations anticipate a large increase in $|\Delta \varepsilon|$ from MM-M6]-MM to $M$ M-M6][6]-MM, but only minor differences in $\varepsilon$. These disparities in $|\Delta \varepsilon|$ and similarities in $\varepsilon$ are exhibited experimentally by $\mathbf{W H}[6]$ and $\mathbf{W H}[6][6]$. Details concerning the TDDFT calculations and the plotting of the predicted spectra can be found in Sections II and VI. 


\section{General Experimental Information}

Synthesis: All reactions were conducted in the designated oven-dried glassware. Schlenk flasks were evacuated and backfilled with argon three times prior to use. Anhydrous tetrahydrofuran was obtained from a Glass Contour solvent system consisting of a Schlenk manifold with purification columns packed with activated alumina and supported copper catalyst. The tetrahydrofuran was dispensed from a Pure-Pac ${ }^{\mathrm{TM}}$ container purchased from Sigma-Aldrich. ReagentPlus ${ }^{\circledR}$ chlorobenzene was used as purchased from Sigma-Aldrich. The 2,7-naphthalenediboronic acid bis(pinacol) ester was prepared using a procedure developed by Schuster et al. ${ }^{1}$ The bromo-hPDI 1 was prepared using a procedure developed by Zhong et al.2 The 1,6-dibromo-7-methoxyperylene-3,4,9,10-tetracarboxylicdiimide was prepared using a procedure developed by Khokhlov et al. ${ }^{3}$ [1, $1^{\prime}$ bis(diphenylphosphino)ferrocene]dichloropalladium(II) was used as purchased from Sigma-Aldrich. ReagentPlus ${ }^{\circledR}$ potassium carbonate was used as purchased from Sigma-Aldrich. All remaining reagents were purchased from Sigma-Aldrich and used without additional purification, unless noted otherwise. SATCO Hi-Pro Spiral 55 W Bright White (3700 lumens) compact fluorescent lamps (CFLs) were used in the preparation of 3 . EverWatt $150 \mathrm{~W}$ light-emitting diode (LED) corn light bulbs (21892 lumens, $4000 \mathrm{~K}$ ) adapted with Mansa Lighting E39/E40-to-E26 bases were used in the preparation of WH[6] and WH[6][6]. Additional details concerning the photoreaction setup are provided in Figure S7 in Section III.

Purification: Automated chromatography was performed using a Teledyne Isco Combiflash $\circledast$ Rf200 and RediSep ${ }^{\circledR}$ normal-phase silica flash columns. Certified ACS hexanes, dichloromethane, and ethyl acetate from Fisher Scientific were used as eluents. Preparative thin-layer chromatography (TLC) employed Silicycle SiliaPlate ${ }^{\mathrm{TM}}$ Glass Backed TLC silica gel plates, $60 \AA, 20 \times 20 \mathrm{~cm}, 2000 \mu \mathrm{m}$ thickness, F-254 indicator.

Chiral Resolution: Racemic WH[6] and WH[6][6] were resolved into their enantiomers by preparative HPLC using a Waters Prep150 LC System equipped with a UV-vis detector, an automated fraction collector, and a CHIRALPAK ${ }^{\circledR}$ IA - amylose tris(3,5-dimethylphenylcarbamate) immobilized on $5 \mu \mathrm{m}$ silica gel - column $\left(21 \mathrm{~mm}\right.$ I.D. $\times 250 \mathrm{~mm}$ ). Optima ${ }^{\text {TM }}$ dichloromethane from Fisher Scientific and HPLC-grade hexanes from Millipore-Sigma were used as eluents. Additional resolution parameters are provided in the caption of Figure S1 in Section I.

UV-Visible Absorbance: The absorbance spectra in Figures $3 \mathrm{~b}$ and $\mathrm{S} 2$ were recorded by a Jasco V750 spectrophotometer. The absorbances of solutions of WH[6] and WH[6][6] in dichloromethane adhere strictly to the Beer-Lambert relation for concentrations of $10^{-6} M$ and a path length of $1 \mathrm{~cm}$ (Figure S42 in Section VII). Each solution was measured in a sealed quartz cuvette.

Fluorescence: The fluorescence spectra in Figure $\mathrm{S} 2$ were recorded at room temperature using a Jasco FP-8300 spectrofluorometer. The solution of WH[6] in dichloromethane $\left(10^{-6} \mathrm{M}\right)$ was photoexcited at $390 \mathrm{~nm}, 417 \mathrm{~nm}, 536 \mathrm{~nm}$, and $568 \mathrm{~nm}$; in all cases, the same emission profile was observed $\left(\lambda_{\max }=578 \mathrm{~nm}\right)$. The solution of $\mathbf{W H}[6][6]$ in dichloromethane $\left(10^{-6} \mathrm{M}\right)$ was photoexcited at $382 \mathrm{~nm}, 407 \mathrm{~nm}, 536 \mathrm{~nm}$, and $567 \mathrm{~nm}$; in all cases, the same emission profile was observed $\left(\lambda_{\max }=\right.$ $576 \mathrm{~nm})$. 
Electronic Circular Dichroism: The ECD spectra in Figure 3a were recorded using a Chirascan V100 spectropolarimeter from Applied Photophysics. A 10-mm path-length high-precision cell made of Quartz SUPRASIL® from Hellma Analytics was used in the collection of the spectra.

NMR Spectroscopy: ${ }^{1 \mathrm{H}-N M R}$ spectra were recorded on a Bruker $400 \mathrm{MHz}$ or $500 \mathrm{MHz}$ spectrometer. ${ }^{13} \mathrm{C}-\mathrm{NMR}$ were recorded on a Bruker $126 \mathrm{MHz}$ spectrometer with complete proton decoupling. Chemical shifts for protons are reported in parts per million (ppm) downfield from tetramethylsilane (TMS) and were referenced to residual protium in the NMR solvent $\left(\mathrm{C}_{2} \mathrm{H}_{2} \mathrm{Cl}_{4}, \delta 6.00\right) .{ }^{4}$ Chemical shifts for carbon are reported in ppm downfield from TMS and were referenced to the carbon resonances of the NMR solvent $\left(\mathrm{C}_{2} \mathrm{D}_{2} \mathrm{Cl}_{4}, \delta\right.$ 73.78). ${ }^{4}{ }^{1} \mathrm{H}-\mathrm{NMR}$ data are represented as follows: chemical shift, multiplicity $(\mathrm{s}=$ singlet, $\mathrm{bs}=$ broad singlet, $\mathrm{d}=$ doublet, $\mathrm{bd}=$ broad doublet, $\mathrm{m}=$ multiplet, and $\mathrm{bm}=$ broad multiplet), coupling constants in $\mathrm{Hz}$, and integration. Most ${ }^{1 \mathrm{H}}$ - and ${ }^{13} \mathrm{C}-\mathrm{NMR}$ spectra were recorded at elevated temperatures (noted in $\mathrm{K}$ ) to enhance peak resolution, particularly in the aromatic region. Peaks corresponding to the numerous aromatic carbon atoms in the reported compounds often overlap, thereby reducing the number of observed peaks relative to the number of anticipated peaks. The NMR spectra can be found in Section IV. The spectra were recorded at the temperatures noted in Section III and in the captions of the figures in Section IV.

The alkyl tails of $\mathbf{W H}[6]$ and $\mathbf{W H}[6][6]$ obscure the spectroscopic fine details that are needed for a conformational analysis by NMR spectroscopy. The chiral-to-meso inversion of $\mathbf{h P D I}_{\mathbf{2}}\left(\Delta \mathrm{G}_{298 \mathrm{~K}}=10.9\right.$ $\mathrm{kcal} / \mathrm{mol}$; see Figure 4 in the main text) occurs on the microsecond timescale at room temperature, whereas the rotation of the $\mathrm{CH}\left(\mathrm{C}_{5} \mathrm{H}_{11}\right)_{2}$ tail about the $\mathrm{N}-\mathrm{C}$ bond of $\mathrm{PDI}$ requires, at least, milliseconds. ${ }^{5}$ The resultant rotamers of $\mathbf{h P D I}_{2}$ have unique proton resonances, which results in substantial signal broadening in the NMR spectra at room temperature. ${ }^{2}$ The same broadening for $\mathbf{W H}[6]$ and $\mathbf{W H}[6][6]$ was observed at $300 \mathrm{~K}$ (Figures S10 and S12 in Section IV), whose reduced symmetry relative to $\mathbf{h P D I}_{2}$ proliferates the number of rotamers; thus, the relative quantities of the dominant conformations of $\mathbf{W H}[6]$ and $\mathbf{W H}[6][6]$ cannot be readily assessed by NMR.

High-Resolution Mass Spectrometry (HRMS): HRMS was conducted using a Waters XEVO G2XS instrument equipped with a UPC2 2 SFC inlet, electrospray (ESI) and atmospheric pressure chemical (APCI) ionization, and a QToF mass spectrometer. For large compounds, HRMS was performed using a Bruker ultrafleXtreme MALDI-TOF/TOF with a frequency-tripled Nd:YAG laser (355 nm), linear and reflector modes, and Precursor lon Selector and LIFT technologies for MS/MS analysis.

Single-Crystal X-Ray Structure Determination: Data for WH[6] were collected on an Agilent SuperNova diffractometer using mirror-monochromated $\mathrm{Cu} K \alpha$ radiation. A 1,2,4-trichlorobenzene solution of racemic WH[6] ( 1 mg in $\sim 2 \mathrm{~mL})$ was diluted with acetonitrile by vapor diffusion to afford fragile red blades (Figure S43 in Section VIII). The crystals lost their solvent rapidly when removed from the mother liquor, so it was necessary to continuously cool the microscope slide with dry ice before mounting the crystal. The crystal was mounted on a glass fiber using Fomblin oil. Data were collected at $100 \mathrm{~K}$. Data collection, integration and scaling (ABSPACK) were performed in CrysAlisPro. 6 Structure solution was performed by intrinsic phasing in ShelXT.7 Subsequent refinement was performed by full-matrix least-squares on $\mathrm{F}^{2}$ in ShelXL. ${ }^{8}$ Olex2 ${ }^{9}$ was used for viewing and to prepare CIF files. PLATON10 was used to model disordered solvent by the SQUEEZE11 method. The thermal ellipsoid plot of WH[6] (Figure S43) was prepared in CrystalMaker. ${ }^{12}$ 
The aromatic core of $\mathbf{W H}[6]$ was identified readily in the initial solution obtained from ShelXT, but the refinement was not stable. Thus, we measured all 1,2- and 1,3- distances in a DFT structure of the molecule and supplied those distances as restraints for the SCXRD refinement. The geometry was additionally stabilized by restraining small parts (halves of the PDI subunits) to be approximately flat. When a reasonable model of the core had been obtained, it was possible to locate the undecyl side chains, although we note that their structures are certainly disordered and the quality of the data did not permit us to identify multiple independent positions. Finally, all $\mathrm{H}$ atoms were placed in calculated positions and refined with riding isotropic ADPs and coordinates. Non- $\mathrm{H}$ atoms were refined with anisotropic ADPs stabilized by global RIGU and SIMU restraints. Disordered solvent was modeled as a diffuse contribution to the overall scattering using SQUEEZE.

The crystallographic data for WH[6] are given in Section VIII.

Density Functional Theory and Time-Dependent Density Functional Theory Calculations: The DFT geometric optimizations, transition-state search, and thermochemical calculations were executed by Jaguar, version 8.3, Schrodinger, Inc., New York, NY, 2014. ${ }^{13}$ The geometries of the ground-state $\pi$-conformations of $\mathbf{h P D I}_{2}, \mathbf{W H}[6]$, and $\mathbf{W H}[6][6]$ were optimized without symmetry restrictions in the gas phase using the B3LYP functional and the $6-31 G^{* *}$ basis set. Subsequent thermochemical singlepoint calculations (B3LYP/6-31G ${ }^{* *}$ ) using the optimized ground-state geometries of $\mathbf{h P D I} \mathbf{2}_{2}$ and $\mathbf{W H}$ [6] in the gas phase provided the Gibbs free energies at $298.15 \mathrm{~K}$ and $1 \mathrm{~atm}$. The helical-to-meso transition-state geometry of $\mathbf{h P D I} \mathbf{2}_{2}$ was identified using a QST search at the B3LYP/6-31G** level of theory. The subsequent thermochemical single-point calculation (B3LYP/6-31G**) using this transition-state geometry in the gas phase returned a single imaginary frequency. The corresponding vibration inverts the flattened [4] helicene within $\mathbf{h P D I} \mathbf{2}_{\mathbf{2}}$ by sliding the two cove hydrogen atoms past one another. This thermochemical calculation also provided the Gibbs free energy of the transition state of $\mathbf{h P D I}_{2}$ at $298.15 \mathrm{~K}$ and $1 \mathrm{~atm}$. The Cartesian coordinates and thermochemical properties of the calculated structures are provided in Section V.

The $\mathrm{CH}\left(\mathrm{C}_{5} \mathrm{H}_{11}\right)_{2}$ imide tails were substituted with methyl groups in the DFT models to expedite the calculations. These substitutions should not significantly alter the optimized geometries and concomitant classification of the $\pi$-conformations of WH[6] and WH[6][6] into three energy tiers (see Figure 5 in the main text), as the alkyl tails can readily shift to accommodate the $\pi$-surface. For instance, PP-M[6]-PP of WH[6] orients the alkyl tails of its terminal PDI subunits directly into one another, which might be expected to effectively eliminate its presence in solution; still, $\boldsymbol{P P}-\boldsymbol{M}$ 6]-PP was the sole conformation (along with its enantiomer) to crystallize from a room-temperature solution of WH[6] (see Figure 6 in the main text), and its $\pi$-surface from SCXRD largely aligns with its DFToptimized geometry (see Figure S44 in Section VIII). Thus, truncating the $\mathrm{CH}\left(\mathrm{C}_{5} \mathrm{H}_{11}\right)_{2}$ tails does not invalidate the classification of the ten conformations of WH[6] and WH[6][6] into three energy tiers; however, the alkyl tails shift the Gibbs free energies of the conformations within each tier and, in turn, the relative amounts of these $\pi$-conformations.

The TDDFT excited-state calculations were executed by Gaussian 16, revision A. 03, Gaussian, Inc., Wallingford, CT, 2016. ${ }^{14}$ The ground-state gas-phase optimized geometries (B3LYP/6-31G**) of the three dominant left-handed conformations of WH[6] (i.e., MM-M[6]-MM, MM-M[6]-PP, and PP-M[6]$P P$ ) and WH[6][6] (i.e., MM-M[6][6]-MM, MM-M[6][6]-PP, and PP-M[6][6]-PP) were used in these calculations. The 100 lowest-energy singlet excited states were calculated for each conformation in 
the gas phase at the CAM-B3LYP/6-31++G** level of theory (using the following keywords: TD=full, singlet, Nstates $=100$, and integral=ultrafinegrid). The corresponding UV-visible absorbance and ECD spectra were generated at the purely electronic level by importing the Gaussian output files into SpecDis, version 1.71, Berlin, Germany, 2017.15 The spectra were rendered using Gaussian bandshapes $(\sigma=0.13 \mathrm{eV})$ and dipole length formalism. Larger bandwidths (i.e., $\sigma=>0.13 \mathrm{eV})$ improved (only slightly) the degree of overlap between the predicted and experimental spectra, as quantified by SpecDis; however, larger bandwidths also reduced the resolution (i.e., fewer distinct bands) of smaller features in the ECD spectra. Since several of these features (such as those at wavelengths $>450 \mathrm{~nm}$ ) most distinguish the spectra of different conformations from one another, the smaller bandwidth was retained. A positive wavelength shift of $56 \mathrm{~nm}$ was also applied to the predicted UV-visible absorbance and ECD spectra. This shift aligned the large bisignate bands exhibited by all conformations (from $\sim 380-430 \mathrm{~nm}$ in the shifted ECD spectra) with the corresponding bisignate bands in the experimental ECD spectra of WH[6] and WH[6][6]. The molar absorptivities and molar ECD of the predicted spectra were scaled automatically by SpecDis to fit the experimental spectra. When predicted and experimental spectra are superimposed in a figure, the predicted spectral intensities are scaled and labeled as "Absorbance" or "ECD" (e.g., see Figure 7 in the main text). The scaling factors are reported in the captions of Figures S36-S41 in Section VI. When not superimposed on an experimental spectrum, the predicted spectra are not scaled. These spectra are labeled as " $\varepsilon$ " or " $\Delta \varepsilon$ " (e.g., see Figure S5). Additional information regarding the TDDFT-calculated excited states of WH[6] and WH[6][6] can be found in Section VI. 


\section{Synthetic Procedures and Characterization Data}

Scheme S1. Overview of the synthesis of WH[6] $\left(\mathrm{R}=\mathrm{CH}\left(\mathrm{C}_{5} \mathrm{H}_{11}\right)_{2}\right)$
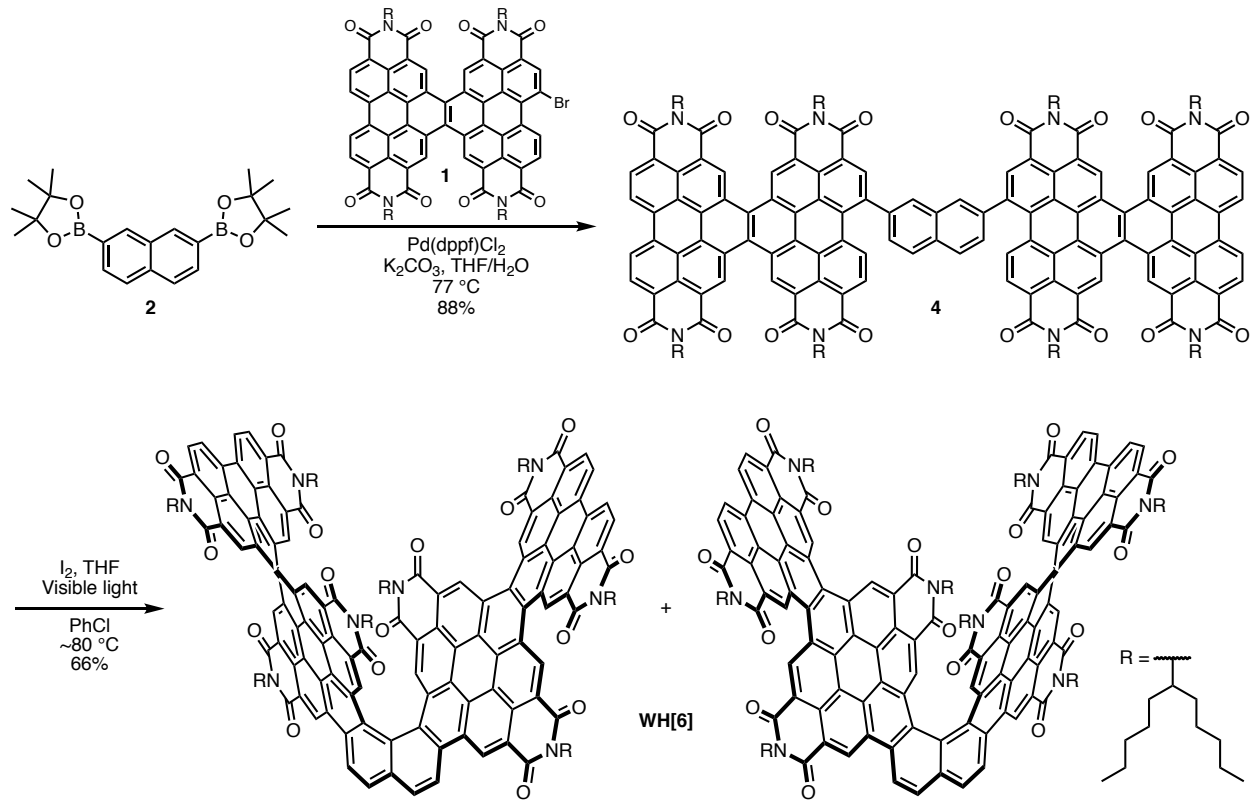

Scheme S2. Overview of the synthesis of WH[6][6] $\left(\mathrm{R}=\mathrm{CH}\left(\mathrm{C}_{5} \mathrm{H}_{11}\right)_{2}\right)$
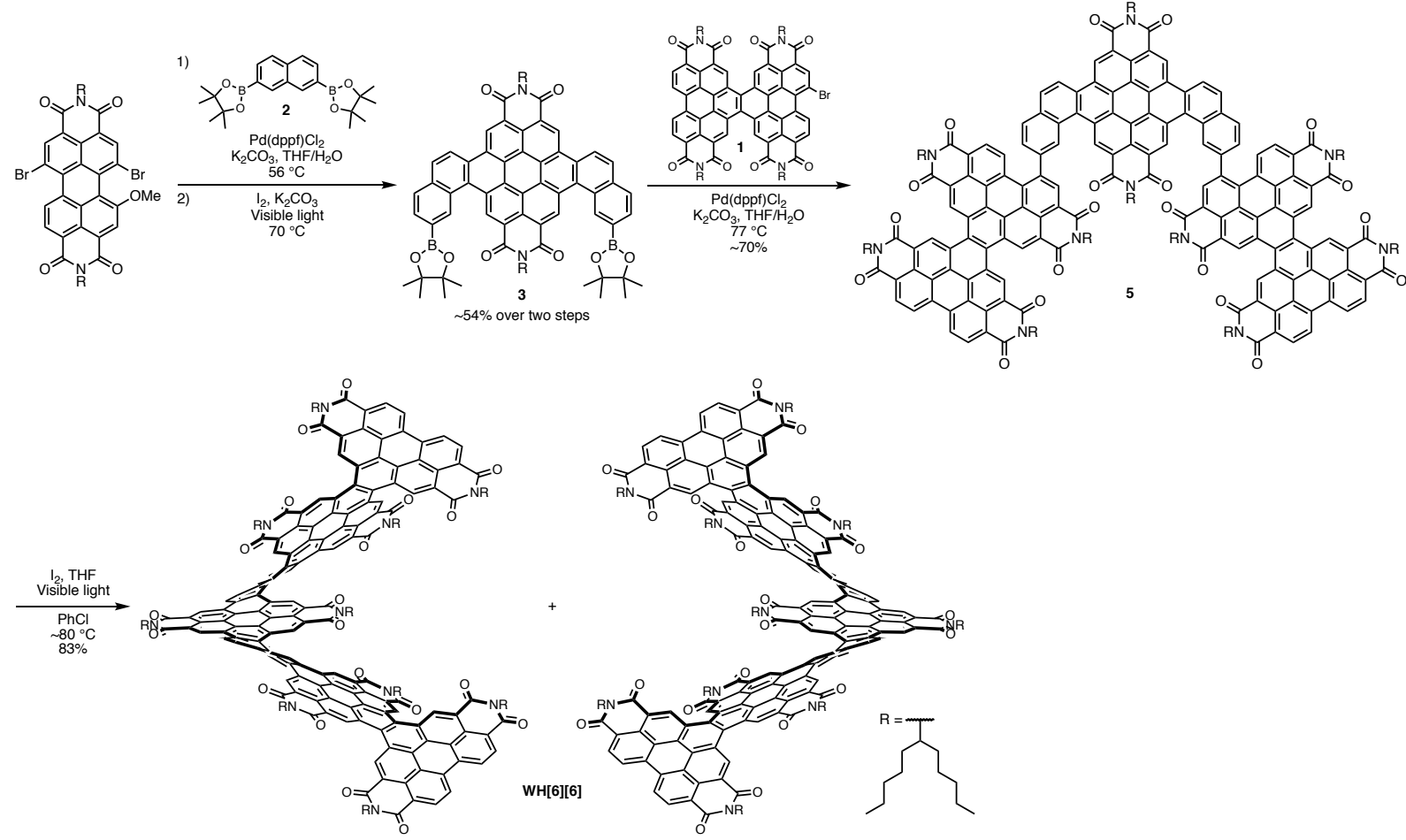


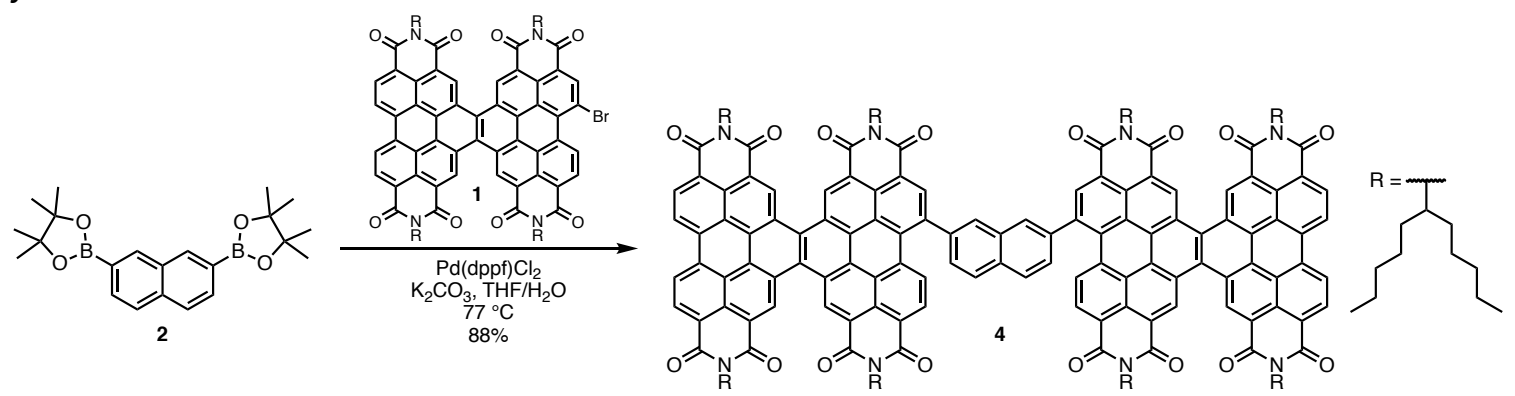

To a 10-mL Schlenk flask equipped with a stir bar and rubber septum, 2 (31.4 mg, 0.0826 mmol, 1 equiv.), 1 (261 mg, $0.174 \mathrm{mmol}, 2.11$ equiv.), potassium carbonate (290. $\mathrm{Mg}, 2.10 \mathrm{mmol}, 25.4$ equiv.), and [1,1'-bis(diphenylphosphino)ferrocene]dichloropalladium (5.4 mg, $0.0074 \mathrm{mmol}, 9.0$ mol\%) were added. In a separate flask, $4.0 \mathrm{~mL}$ of tetrahydrofuran and $1.8 \mathrm{~mL}$ of water were mixed and sparged with argon; $4.0 \mathrm{~mL}$ of this solvent were injected into the Schlenk flask. While stirring, the resultant mixture was sparged with argon for $10 \mathrm{~min}$. While maintaining a positive flow of argon, the septum was exchanged with a greased glass stopcock. The mixture was sealed under argon and stirred at $77^{\circ} \mathrm{C}$ for $14 \mathrm{~h}$. The resultant mixture was diluted with dichloromethane, washed with brine in a separatory funnel, dried over magnesium sulfate, and filtered. The solvents were removed under reduced pressure. Purification by column chromatography on silica gel (gradient of $100 \%$ hexanes to $100 \%$ dichloromethane) yielded 4 as a red powder $(216 \mathrm{mg}, 0.0730 \mathrm{mmol}, 88 \%)$. ${ }^{1} \mathrm{H} \mathrm{NMR}(500 \mathrm{MHz}$, $\left.\mathrm{C}_{2} \mathrm{D}_{2} \mathrm{Cl}_{4}, 353 \mathrm{~K}\right) \delta 10.40(\mathrm{bd}, J=10.3 \mathrm{~Hz}, 4 \mathrm{H}), 10.37(\mathrm{bd}, J=13.2 \mathrm{~Hz}, 4 \mathrm{H}), 9.51(\mathrm{~d}, J=8.3 \mathrm{~Hz}, 4 \mathrm{H})$, 9.32 (bs, 2H), 9.26 (d, J=8.1 Hz, 4H), 8.86 (bs, 2H), 8.72 (bs, 2H), 8.60 (bs, 2H), 8.38 (bs, 2H), 7.93 (bs, $2 \mathrm{H}), 5.37(\mathrm{bm}, 8 \mathrm{H}), 2.41(\mathrm{bm}, 16 \mathrm{H}), 2.07(\mathrm{bm}, 16 \mathrm{H}), 1.6$ to 1.3 (many intersecting alkyl signals; difficult to integrate precisely due to overlap with water signal), $0.93(\mathrm{bm}, 48 \mathrm{H}) .{ }^{13 \mathrm{C} \mathrm{NMR}}(126 \mathrm{MHz}$, $\left.\mathrm{C}_{2} \mathrm{D}_{2} \mathrm{Cl}_{4}, 363 \mathrm{~K}\right) \delta 164.16,141.96,141.78,135.82,135.08,133.88,133.56,133.28,132.73,131.84$, $130.31,130.18,129.94,129.02$, 128.06, 127.68, 127.24, 127.21, 126.81, 126.79, 126.77, 126.45, $126.41,126.35,126.33,126.19,125.98,125.90,125.67,125.65,125.35,124.24,123.89,123.83$, 123.67, 122.88, 122.35, 122.10, 55.21, 55.11, 55.04, 32.41, 31.54, 26.53, 22.27, 13.74. HRMS (MALDI-TOF): calculated $\mathrm{m} / \mathrm{z}$ for $\left[\mathrm{C}_{198} \mathrm{H}_{212} \mathrm{~N}_{8} \mathrm{O}_{16}\right]-$ is 2959.6093 ; found 2959.6075 .

\section{b. Synthesis of $\mathbf{W H}[6]$}

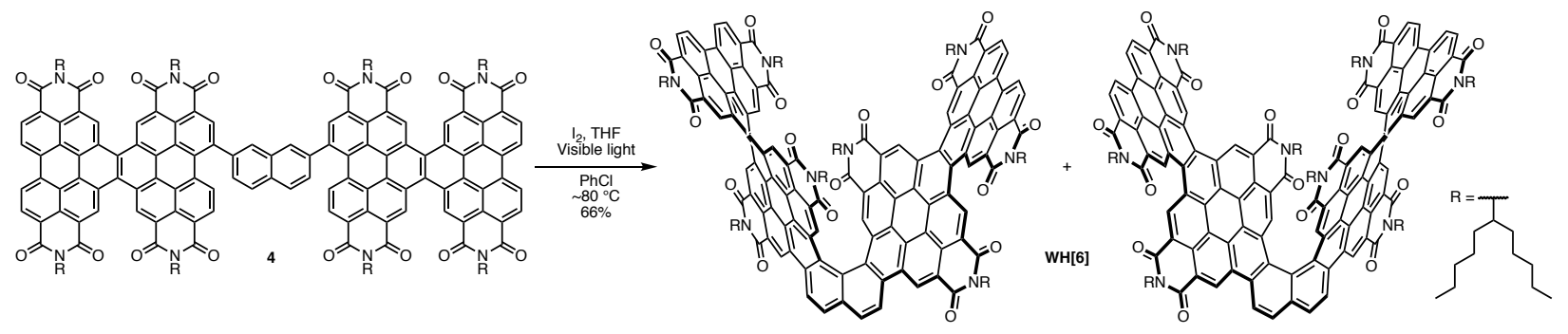

In a $60-\mathrm{mL}$ Ace pressure tube (outer diameter of $25.4 \mathrm{~mm}$ and length of $305 \mathrm{~mm}$ ) equipped with a stir bar, 4 (51.2 mg, $0.0173 \mathrm{mmol}, 1$ equiv.), iodine (21.3 mg, $0.0839 \mathrm{mmol}, 4.85$ equiv.), and tetrahydrofuran (140 mg, $1.9 \mathrm{mmol}, 110$ equiv.) were dissolved in $51 \mathrm{~mL}$ of chlorobenzene. The tube was sealed with a threaded PTFE plug. While stirring vigorously, the solution was irradiated with four 150-W white-light LED corn light bulbs within a vented cabinet (see Figure S7 for the reaction setup) for $48.5 \mathrm{~h}$. Within $1 \mathrm{~h}$ of being turned on, the light bulbs heated the air in the cabinet to a maximum 
temperature of $80^{\circ} \mathrm{C}$. The temperature remained at $\sim 80^{\circ} \mathrm{C}$ for the remainder of the reaction. The tube was cooled to room temperature, and the remaining iodine was quenched by stirring the reaction solution over an excess of solid sodium thiosulfate pentahydrate. This mixture was filtered through Celite, which was extracted with dichloromethane until colorless. The solvent was removed under reduced pressure. The resultant crude material was dissolved in a small volume of dichloromethane and loaded onto two 2000- $\mu \mathrm{m}$ silica-gel preparative-TLC plates. Two developments (7:3 [v/v] mixture of dichloromethane/hexanes, then $4: 1$ [v/v] mixture of dichloromethane/hexanes) provided WH[6] as a bright red solid (33.8 mg, $0.0114 \mathrm{mg}, 66 \%)$. ${ }^{1} \mathrm{H}$ NMR $\left(500 \mathrm{MHz}, \mathrm{C}_{2} \mathrm{D}_{2} \mathrm{Cl}_{4}, 360 \mathrm{~K}\right) \delta 11.17(\mathrm{~s}, 2 \mathrm{H})$, 10.91 (s, 2H), 10.69 (s, 2H), 10.55 (s, 2H), 10.37 (s, 2H), 10.10 (d, J = 9.1 Hz, 2H), 9.64 (s, 2H), 9.53 (d, $J=8.6 \mathrm{~Hz}, 2 \mathrm{H}), 9.51(\mathrm{~d}, J=8.7 \mathrm{~Hz}, 2 \mathrm{H}), 9.30(\mathrm{~d}, J=8.2 \mathrm{~Hz}, 2 \mathrm{H}), 9.23(\mathrm{~d}, J=8.2 \mathrm{~Hz}, 2 \mathrm{H}), 9.10$ (d, $J=8.6 \mathrm{~Hz}, 2 \mathrm{H}), 5.64(\mathrm{~m}, 2 \mathrm{H}), 5.44(\mathrm{~m}, 2 \mathrm{H}), 5.24(\mathrm{~m}, 2 \mathrm{H}), 4.31(\mathrm{bm}, 2 \mathrm{H}), 2.63(\mathrm{bm}, 4 \mathrm{H}), 2.47(\mathrm{bm}$, $4 \mathrm{H}), 2.27(\mathrm{bm}, 8 \mathrm{H}), 2.13(\mathrm{bm}, 4 \mathrm{H}), 1.93(\mathrm{bm}, 4 \mathrm{H}), 1.8$ to -1.0 (many intersecting alkyl signals; difficult to integrate precisely due to overlap with water signal). ${ }^{13} \mathrm{C} \mathrm{NMR}\left(126 \mathrm{MHz}, \mathrm{C}_{2} \mathrm{D}_{2} \mathrm{Cl}_{4}, 363 \mathrm{~K}\right) \delta 164.87$, 164.24, 163.76, 163.04, 133.99, 133.90, 133.81, 133.55, 132.09, 131.47, 130.48, 130.16, 130.04, 129.64, 128.81, 128.24, 127.96, 127.26, 127.14, 126.90, 126.72, 126.61, 126.42, 125.59, 125.47, $125.03,124.99,124.80,124.75,124.54,124.46,124.23,124.16,124.10,123.95,123.68,123.26$, $123.13,123.03,122.78,122.47,122.11,121.87,119.96,55.57,55.17,54.98,53.53,32.77,32.63$, $32.55,32.41,32.37,32.11,31.68,31.57,31.42,31.38,31.11,30.88,30.44,29.44,26.75,26.56$, $26.39,25.34,22.36,22.35,22.32,22.15,22.11,21.51,21.37,13.83,13.76,13.59,12.97$. HRMS (MALDI-TOF): calculated $\mathrm{m} / \mathrm{z}$ for $\left[\mathrm{C}_{198} \mathrm{H}_{208} \mathrm{~N}_{8} \mathrm{O}_{16}\right]-$ is 2955.5780 ; found 2955.6958 .

\section{c. Synthesis of 3}
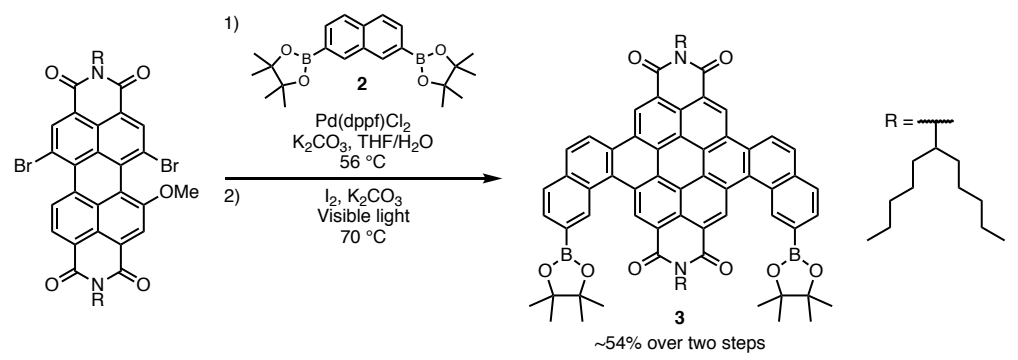

To a 10-mL Schlenk flask equipped with a stir bar and rubber septum, the 1,6-dibromo-7methoxyperylene diimide (128 mg, $0.144 \mathrm{mmol}, 1$ equiv.), 2 (341 mg, $0.897 \mathrm{mmol}, 6.23$ equiv.), [1,1'bis(diphenylphosphino)ferrocene]dichloropalladium (10.6 mg, $0.0145 \mathrm{mmol}, 10.1 \mathrm{~mol} \%$ ), and potassium carbonate (212 mg, $1.53 \mathrm{mmol}, 10.6$ equiv.) were added. The flask was evacuated and backfilled with argon three times. Meanwhile, $10.0 \mathrm{~mL}$ of tetrahydrofuran and $3.4 \mathrm{~mL}$ of water were mixed and sparged with argon; then, $5.0 \mathrm{~mL}$ of this solvent were injected into the Schlenk flask. While stirring, the resultant mixture was sparged with argon for 10 min. Under continuous argon flow, the mixture was stirred at $56^{\circ} \mathrm{C}$ for $18 \mathrm{~h}$.

The mixture was filtered through Celite into a $500-\mathrm{mL}$ single-neck round-bottom flask. The Celite was extracted with dichloromethane until colorless. The solvent was removed under reduced pressure. A large stir bar, iodine (198 $\mathrm{mg}, 0.780 \mathrm{mmol}, 5.42$ equiv.), potassium carbonate (2.0 g, 14 mmol, 97 equiv.), and $127 \mathrm{~mL}$ of chlorobenzene were added to the flask, which was then sealed with a rubber septum. While stirring in subdued light, the mixture was sparged vigorously with nitrogen for $40 \mathrm{~min}$. Under continuous nitrogen flow, the stirring mixture was heated by a pristine oil bath at $70^{\circ} \mathrm{C}$ 
and irradiated with two 55-W white-light CFLs for $16 \mathrm{~h}$. The flask was cooled to room temperature, and the remaining iodine was quenched by stirring the reaction mixture over an excess of solid sodium thiosulfate pentahydrate. This mixture was filtered through Celite, which was extracted with dichloromethane until colorless. The solvent was removed under reduced pressure. Purification by column chromatography on silica gel (gradient of $100 \%$ hexanes to $100 \%$ dichloromethane) yielded a red film, which was washed with acetonitrile to provide 3 as a red-orange powder ( 92.5 mg, 0.0771 mmol, 54\%). ${ }^{1} \mathrm{H} \mathrm{NMR}\left(500 \mathrm{MHz}, \mathrm{C}_{2} \mathrm{D}_{2} \mathrm{Cl}_{4}, 323 \mathrm{~K}\right.$ ) $\delta 11.04$ (bs, $2 \mathrm{H}$ ), 10.77 (bs, 2H), 9.95 (s, 2H), 9.56 $(\mathrm{d}, J=9.1 \mathrm{~Hz}, 2 \mathrm{H}), 8.57(\mathrm{~d}, J=8.9 \mathrm{~Hz}, 2 \mathrm{H}), 8.37(\mathrm{~d}, J=7.9 \mathrm{~Hz}, 2 \mathrm{H}), 8.32(\mathrm{~d}, J=7.8 \mathrm{~Hz}, 2 \mathrm{H}), 5.54$ (bm, 2H), $2.53(\mathrm{bm}, 4 \mathrm{H}), 2.13(\mathrm{bm}, 4 \mathrm{H}), 1.7$ to 1.2 (intersecting alkyl and pinacolato signals; difficult to integrate precisely due to overlap with water signal), $0.89(\mathrm{bm}, 12 \mathrm{H})$. HRMS (ESI+): calculated $\mathrm{m} / \mathrm{z}$ for $\left[\mathrm{C}_{78} \mathrm{H}_{84} \mathrm{~B}_{2} \mathrm{~N}_{2} \mathrm{O}_{8}+\mathrm{Na}\right]+$ is 1221.6329 ; found 1221.6306. ${ }^{1} \mathrm{H}$ NMR also indicated that the isolated powder contained a minor amount of PDI-based contaminant. This contaminant may originate from the presence of the small amount of 1,7-dibromo-6-methoxyperylene diimide - an unresolvable byproduct of the synthesis of the 1,6-dibromo-7-methoxyperylene diimide ${ }^{3}$ - in the starting material. As isolated above, 3 was used in the subsequent reaction (vide infra).

\section{d. Synthesis of 5}

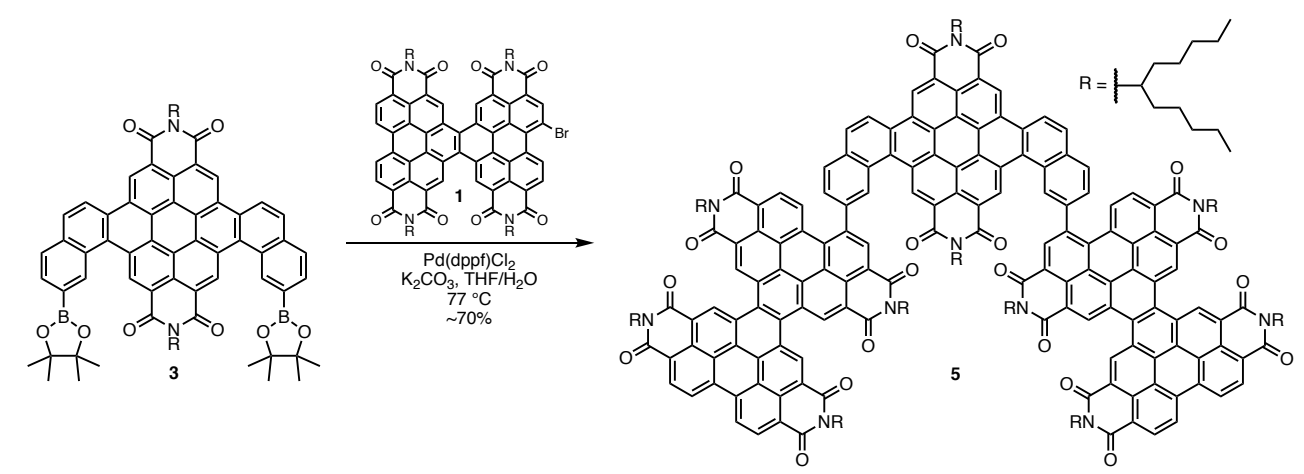

To a 10-mL Schlenk flask equipped with a stir bar and rubber septum, 3 ( $\sim 72.8 \mathrm{mg}, \sim 0.0607$ mmol, 1 equiv.), 1 (187 mg, $0.125 \mathrm{mmol}, 2.06$ equiv.), potassium carbonate (215 mg, $1.56 \mathrm{mmol}, 25.7$ equiv.), and [1,1'-bis(diphenylphosphino)ferrocene]dichloropalladium (4.6 mg, $0.0063 \mathrm{mmol}, 10$. mol\%) were added. The flask was evacuated and backfilled with argon five times. Meanwhile, $7.0 \mathrm{~mL}$ of tetrahydrofuran and $3.0 \mathrm{~mL}$ of water were mixed and sparged with argon; then, $2.5 \mathrm{~mL}$ of this solvent were injected into the Schlenk flask. While stirring, the resultant mixture was sparged with argon for $10 \mathrm{~min}$. While maintaining a positive flow of argon, the septum was exchanged with a greased glass stopcock. The mixture was sealed under argon and stirred at $77^{\circ} \mathrm{C}$ for $16 \mathrm{~h}$. The mixture was filtered through Celite, which was extracted with dichloromethane until colorless. The solvent was removed under reduced pressure. Purification by column chromatography on silica gel (gradient of $100 \%$ hexanes to $100 \%$ of $99: 1$ [v/v] dichloromethane/ethyl acetate) yielded a red film. This film was dissolved in a very small volume of dichloromethane and precipitated with ethyl acetate. The precipitate was collected by filtration and dried in vacuo to provide 5 as a red powder $(\sim 161 \mathrm{mg}$, $\sim 0.0426 \mathrm{mmol}, \sim 70 \%)$. ${ }^{1} \mathrm{H}$ NMR showed numerous overlapping and broadened peaks, which can be attributed to the hindered rotation of the terminal $\pi$-extended $\mathrm{hPDI}_{2}$ subunits, as well as to the hindered rotation of the alkyl chains. Even at elevated temperatures, a well-resolved ${ }^{1} \mathrm{H}-\mathrm{NMR}$ spectrum was not obtained. HRMS (MALDI-TOF): calculated m/z for $\left[\mathrm{C}_{254} \mathrm{H}_{266} \mathrm{~N}_{10} \mathrm{O}_{20}\right]^{-}$is 3778.0176 ; found 3777.9004 . 
HRMS (MALDI-TOF) also detected trace $h \mathrm{PDI}_{2}$ (calculated $\mathrm{m} / \mathrm{z}$ for $\left[\mathrm{C}_{94} \mathrm{H}_{104} \mathrm{~N}_{4} \mathrm{O}_{8}\right]-$ is 1417.7893 ; found 1417.7101), which arises from the hydrodehalogenation of $\mathbf{1}$. As isolated above, $\mathbf{5}$ was used in the subsequent reaction (vide infra).

\section{e. Synthesis of $\mathrm{WH}[6][6]$}

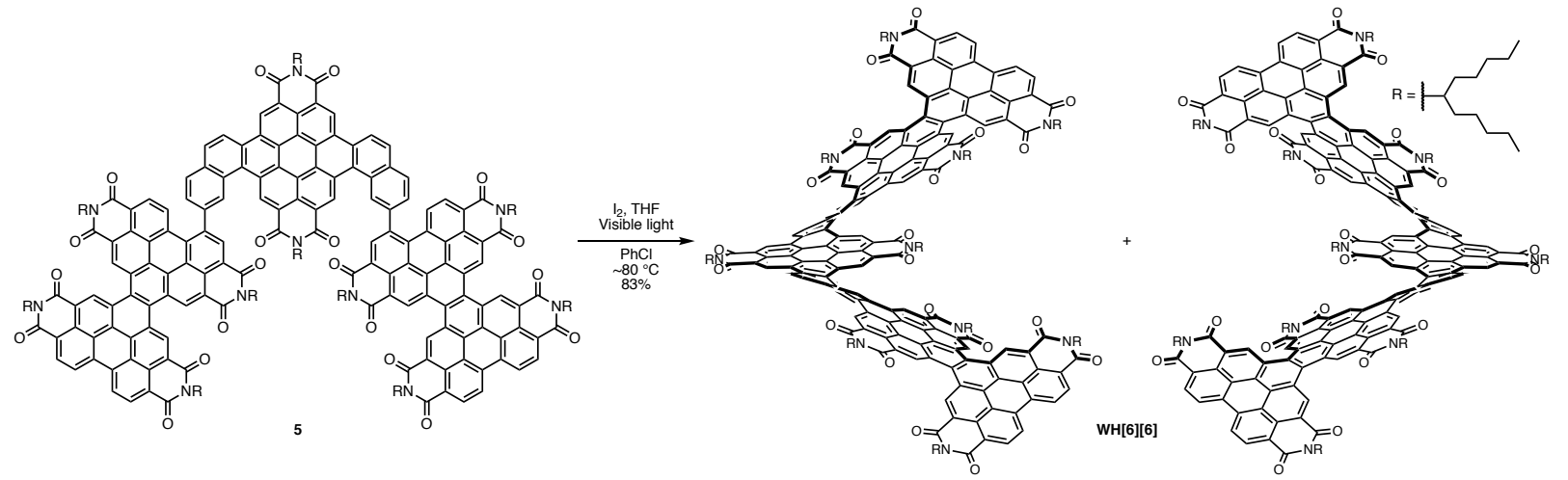

In a 60-mL Ace pressure tube (outer diameter of $25.4 \mathrm{~mm}$ and length of $305 \mathrm{~mm}$ ) equipped with a stir bar, 5 (52.1 mg, $0.0138 \mathrm{mmol}, 1$ equiv.), iodine (14.2 mg, $0.0559 \mathrm{mmol}, 4.05$ equiv.), and tetrahydrofuran (107 mg, $1.48 \mathrm{mmol}, 107$ equiv.) were dissolved in $41 \mathrm{~mL}$ of chlorobenzene. The tube was sealed with a threaded PTFE plug. While stirring vigorously, the solution was irradiated with four $150-W$ white-light LED corn light bulbs within a vented cabinet (see Figure S7 for the reaction setup) for $48 \mathrm{~h}$. Within $1 \mathrm{~h}$ of being turned on, the light bulbs heated the air in the cabinet to a maximum temperature of $80^{\circ} \mathrm{C}$. The temperature remained at $\sim 80^{\circ} \mathrm{C}$ for the remainder of the reaction. The tube was cooled to room temperature, and the remaining iodine was quenched by stirring the reaction solution over an excess of solid sodium thiosulfate pentahydrate. This mixture was filtered through Celite, which was extracted with dichloromethane until colorless. The solvent was removed under reduced pressure. The resultant crude material was dissolved in a small volume of dichloromethane and loaded onto two 2000- $\mu \mathrm{m}$ silica-gel preparative-TLC plates. Development $(4: 1[\mathrm{v} / \mathrm{v}]$ mixture of dichloromethane/hexanes) provided $\mathbf{W H}[6][6]$ as a bright red solid (43.6 mg, $0.0115 \mathrm{mg}, 83 \%) .{ }^{1 \mathrm{H}}$ NMR (400 MHz, $\left.\mathrm{C}_{2} \mathrm{D}_{2} \mathrm{Cl}_{4}, 363 \mathrm{~K}\right) \delta 11.13(\mathrm{~s}, 2 \mathrm{H}), 11.04(\mathrm{~s}, 2 \mathrm{H}), 10.71(\mathrm{~s}, 2 \mathrm{H}), 10.46(\mathrm{~s}, 2 \mathrm{H}), 10.26$ (s, 2H), $10.13(\mathrm{~s}, 2 \mathrm{H}), 10.06(\mathrm{~d}, J=9.0 \mathrm{~Hz}, 2 \mathrm{H}), 9.99(\mathrm{~d}, J=8.9 \mathrm{~Hz}, 2 \mathrm{H}), 9.43(\mathrm{~d}, J=8.4 \mathrm{~Hz}, 2 \mathrm{H}), 9.42$ $(\mathrm{d}, J=8.2 \mathrm{~Hz}, 2 \mathrm{H}), 9.34(\mathrm{~s}, 2 \mathrm{H}), 9.28(\mathrm{~s}, 2 \mathrm{H}), 9.20(\mathrm{~d}, J=8.1 \mathrm{~Hz}, 2 \mathrm{H}), 9.16(\mathrm{~d}, J=8.1 \mathrm{~Hz}, 2 \mathrm{H}), 9.05$ (d, $J=8.4 \mathrm{~Hz}, 2 \mathrm{H}), 9.02(\mathrm{~d}, J=8.4 \mathrm{~Hz}, 2 \mathrm{H}), 5.77(\mathrm{~m}, 1 \mathrm{H}), 5.52(\mathrm{~m}, 2 \mathrm{H}), 5.30(\mathrm{~m}, 2 \mathrm{H}), 5.17(\mathrm{~m}, 2 \mathrm{H})$, $4.10(\mathrm{bm}, 2 \mathrm{H}), 3.16(\mathrm{~m}, 1 \mathrm{H}), 2.9$ to -1.2 (many intersecting alkyl signals; difficult to integrate precisely due to overlap with water signal). ${ }^{13} \mathrm{C}$ NMR (126 MHz, $\left.\mathrm{C}_{2} \mathrm{D}_{2} \mathrm{Cl}_{4}, 363 \mathrm{~K}\right) \delta 165.40,164.91,164.63$, 164.06, 163.70, 163.38, 162.78, 162.15, 133.87, 133.76, 133.68, 133.55, 133.36, 131.83, 131.13, $130.59,130.30,130.01,129.94,129.45,128.68,128.46,128.13,127.84,127.45,127.09,127.02$, $126.93,126.91,126.86,126.66,126.45,126.40,126.38,126.04,125.43,125.28,125.00,124.75$, $124.68,124.55,124.52,124.37,124.10,124.05,124.02,123.99,123.87,123.80,123.56,123.33$, $123.06,122.89,122.80,122.69,122.23,121.68,121.65,121.53,119.45,119.20,55.65,55.41,54.99$, $54.91,53.46,51.77,32.94,32.83,32.63,32.50,32.39,32.32,32.21,32.08,31.85,31.82,31.57$, $31.44,31.40,31.38,31.35,31.12,30.84,30.46,29.74,29.44,29.22,29.12,29.05,26.88,26.61$, $26.42,26.40,26.35,25.23,24.11,23.44,22.44,22.25,22.23,22.19,22.17,22.11,22.09,21.68$, $21.45,20.68,20.06,13.91,13.73,13.71,13.63,13.57,13.56,13.40,13.26,12.07,11.93$. HRMS (MALDI-TOF): calculated $\mathrm{m} / \mathrm{z}$ for $\left[\mathrm{C}_{254} \mathrm{H}_{262} \mathrm{~N}_{10} \mathrm{O}_{20}\right]-$ is 3773.9863 ; found 3774.0637 . 

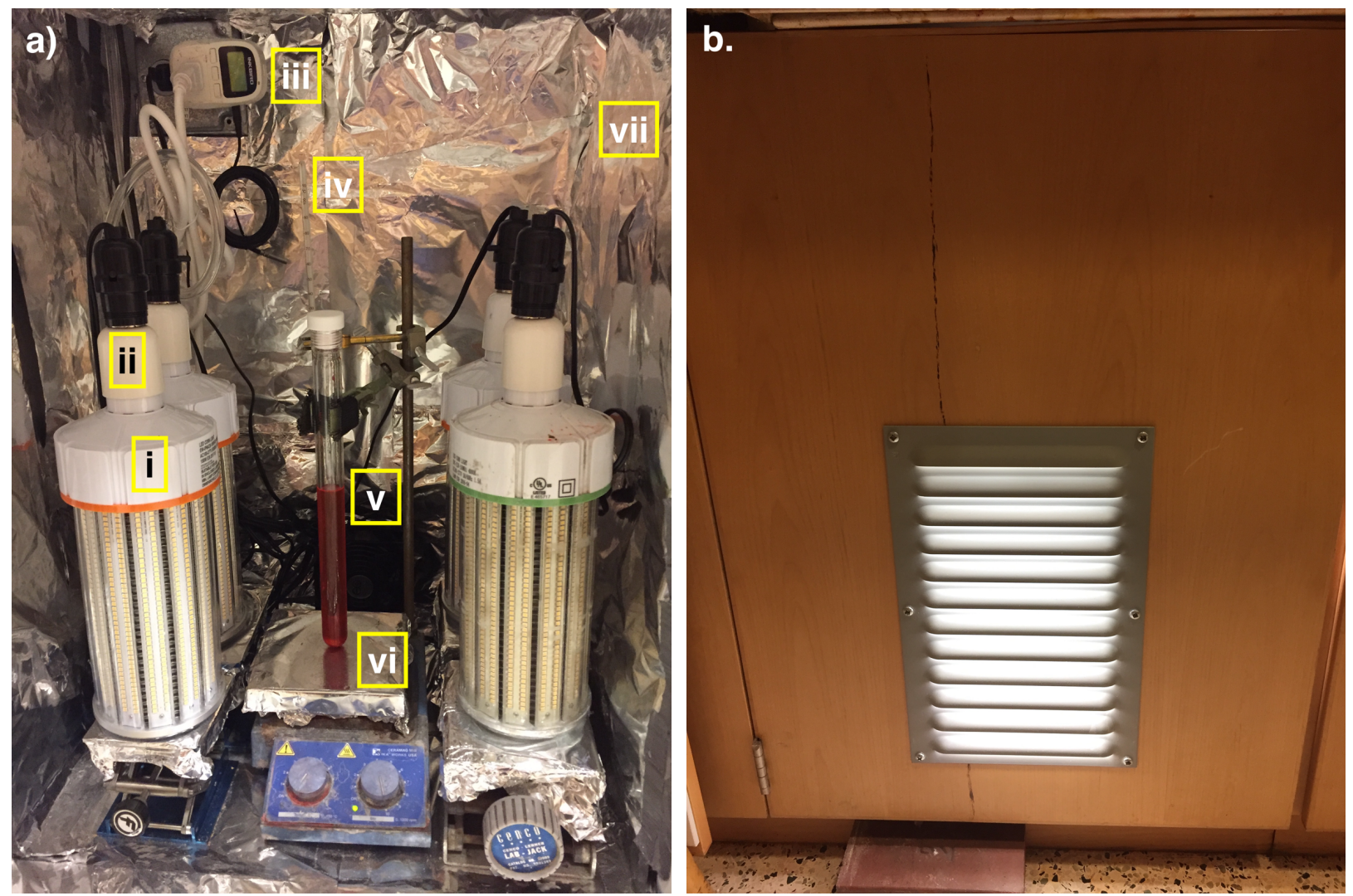

Figure S7. (a) The interior of the photoreaction cabinet (i. EverWatt 150 W LED corn light bulb [21892 lumens, $4000 \mathrm{~K}$; ii. Mansa Lighting E39/E40-to-E26 base; iii. Inkbird C206T $1500 \mathrm{~W}$ heat temperature controller with probe; iv. Thermometer; v. AC Infinity AXIAL 1238 high-speed fan [110 feet ${ }^{3}$ $\mathrm{min}^{-1}$ ] connected to fume hood exhaust port; vi. Stir plate; vii. Aluminum foil). (b) The exterior of the photoreaction cabinet with a reaction in progress. The vent (McMaster-Carr surface mount enclosure louver, steel frame and blades, for 8 inch wide $\times 14$ 1/16 inch high opening) helps to cool the cabinet. 


\section{IV. ${ }^{1} \mathrm{H}-\mathrm{NMR}$ and ${ }^{13} \mathrm{C}-\mathrm{NMR}$ Spectra}

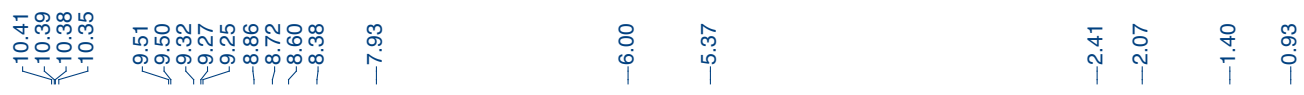
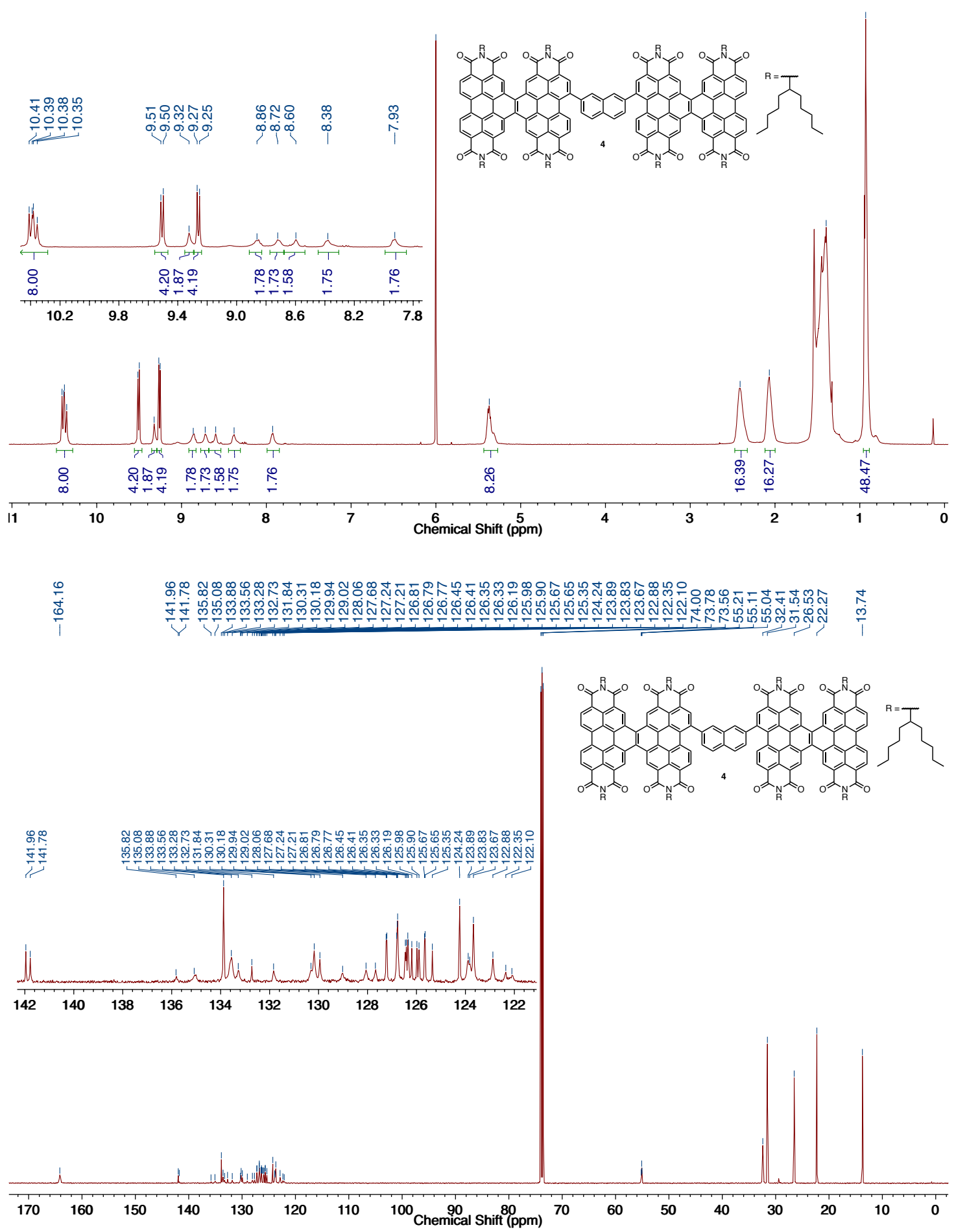

Figure S8. ${ }^{1} \mathrm{H}$ - and ${ }^{13} \mathrm{C}-\mathrm{NMR}$ spectra of 4 in $\mathrm{C}_{2} \mathrm{D}_{2} \mathrm{Cl}_{4}$ at $353 \mathrm{~K}$ and $363 \mathrm{~K}$, respectively. 


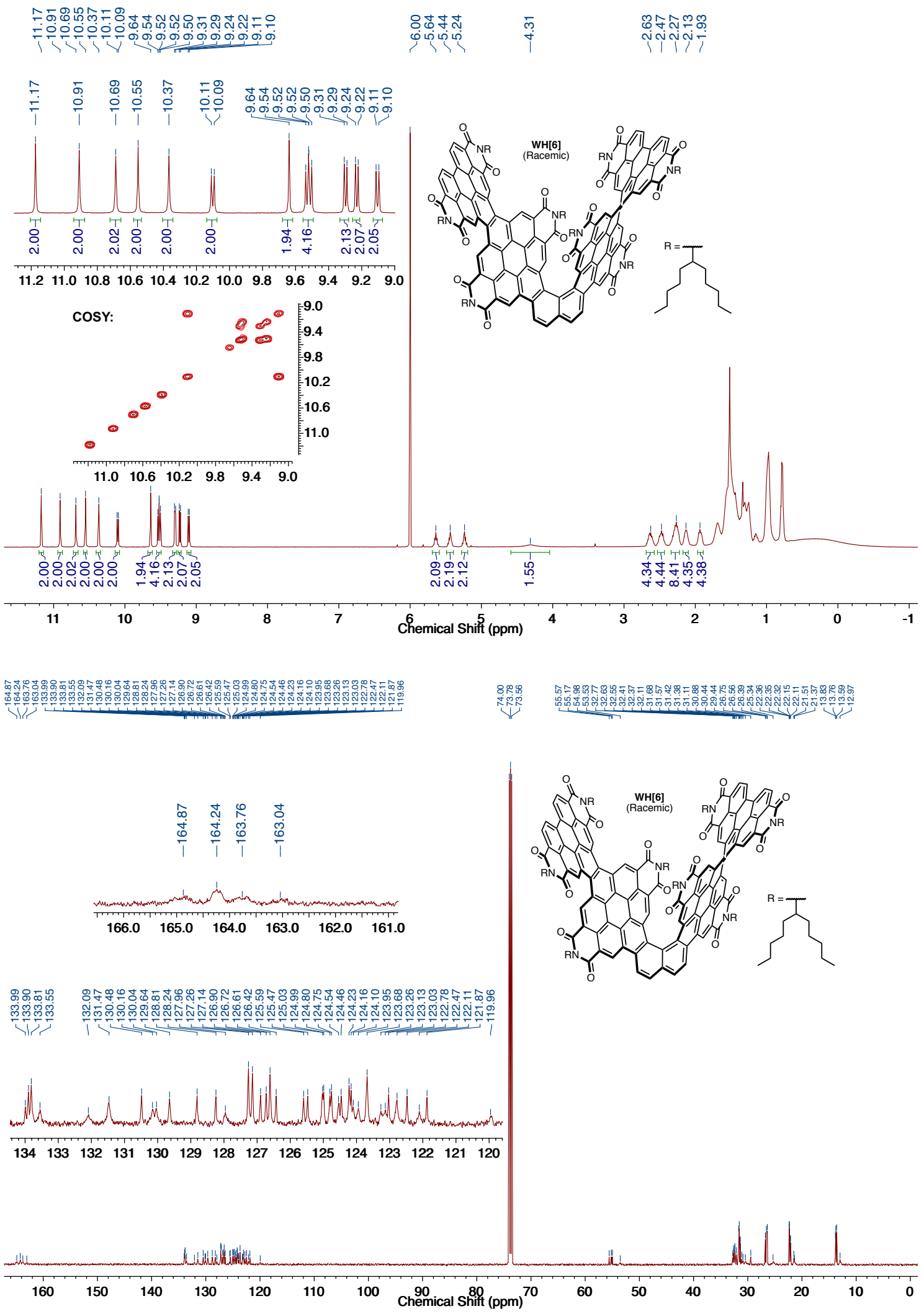

Figure S9. ${ }^{1} \mathrm{H}$ - and ${ }^{13} \mathrm{C}$-NMR spectra of racemic $\mathbf{W H}[6]$ in $\mathrm{C}_{2} \mathrm{D}_{2} \mathrm{Cl}_{4}$ at $360 \mathrm{~K}$ and $363 \mathrm{~K}$, respectively. 


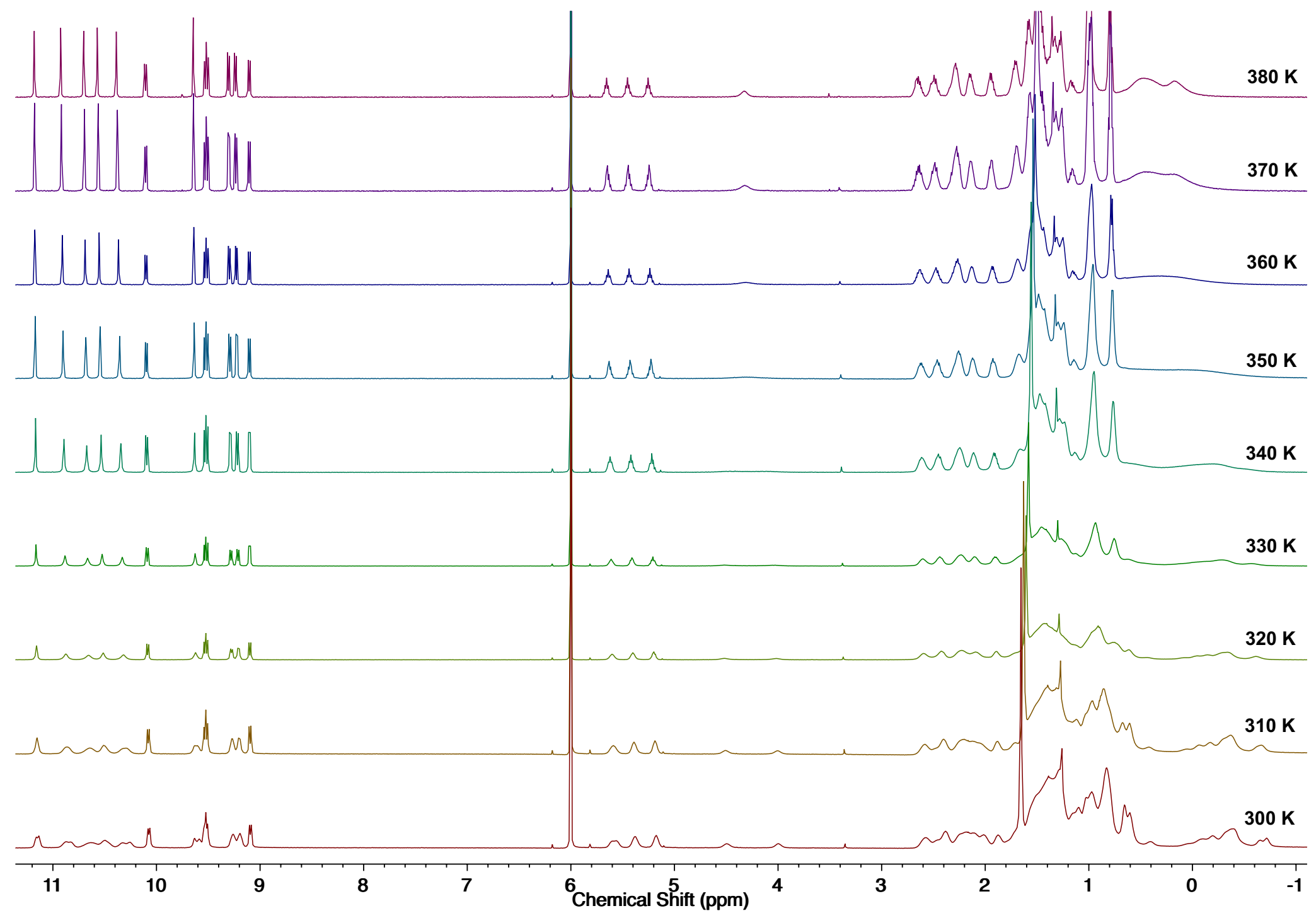

Figure S10. ${ }^{1} \mathrm{H}-\mathrm{NMR}$ spectra of racemic WH[6] in $\mathrm{C}_{2} \mathrm{D}_{2} \mathrm{Cl}_{4}$ from $300-380 \mathrm{~K}$ in increments of $10 \mathrm{~K}$. 


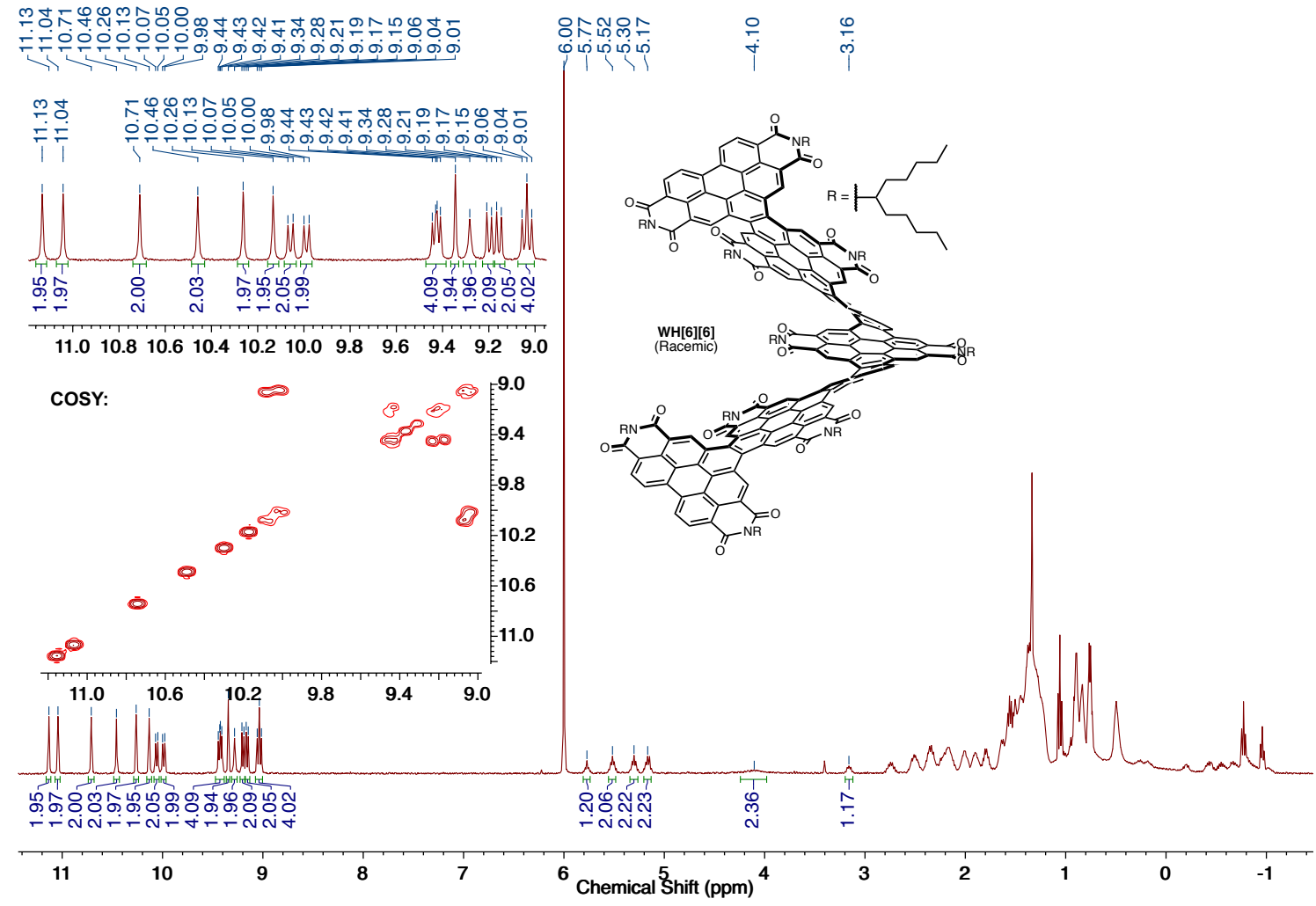

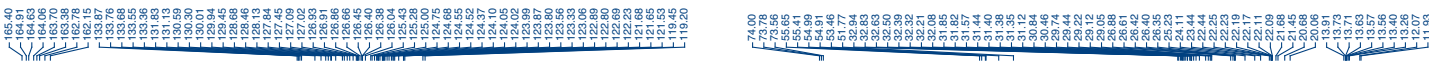

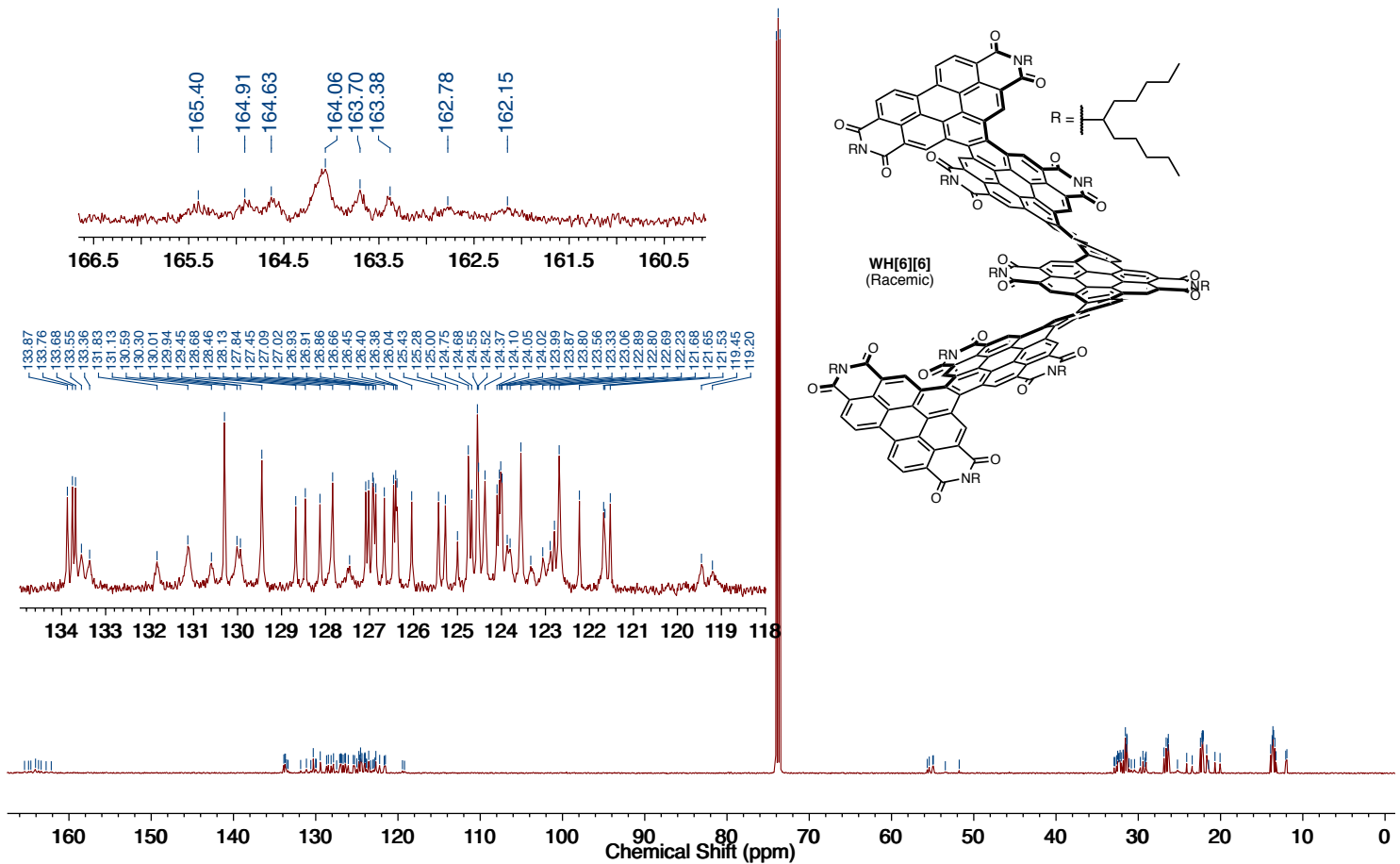

Figure $\mathrm{S} 11 .{ }^{1} \mathrm{H}$ - and ${ }^{13} \mathrm{C}-\mathrm{NMR}$ spectra of racemic $\mathrm{WH}[6][6]$ in $\mathrm{C}_{2} \mathrm{D}_{2} \mathrm{Cl}_{4}$ at $363 \mathrm{~K}$. 


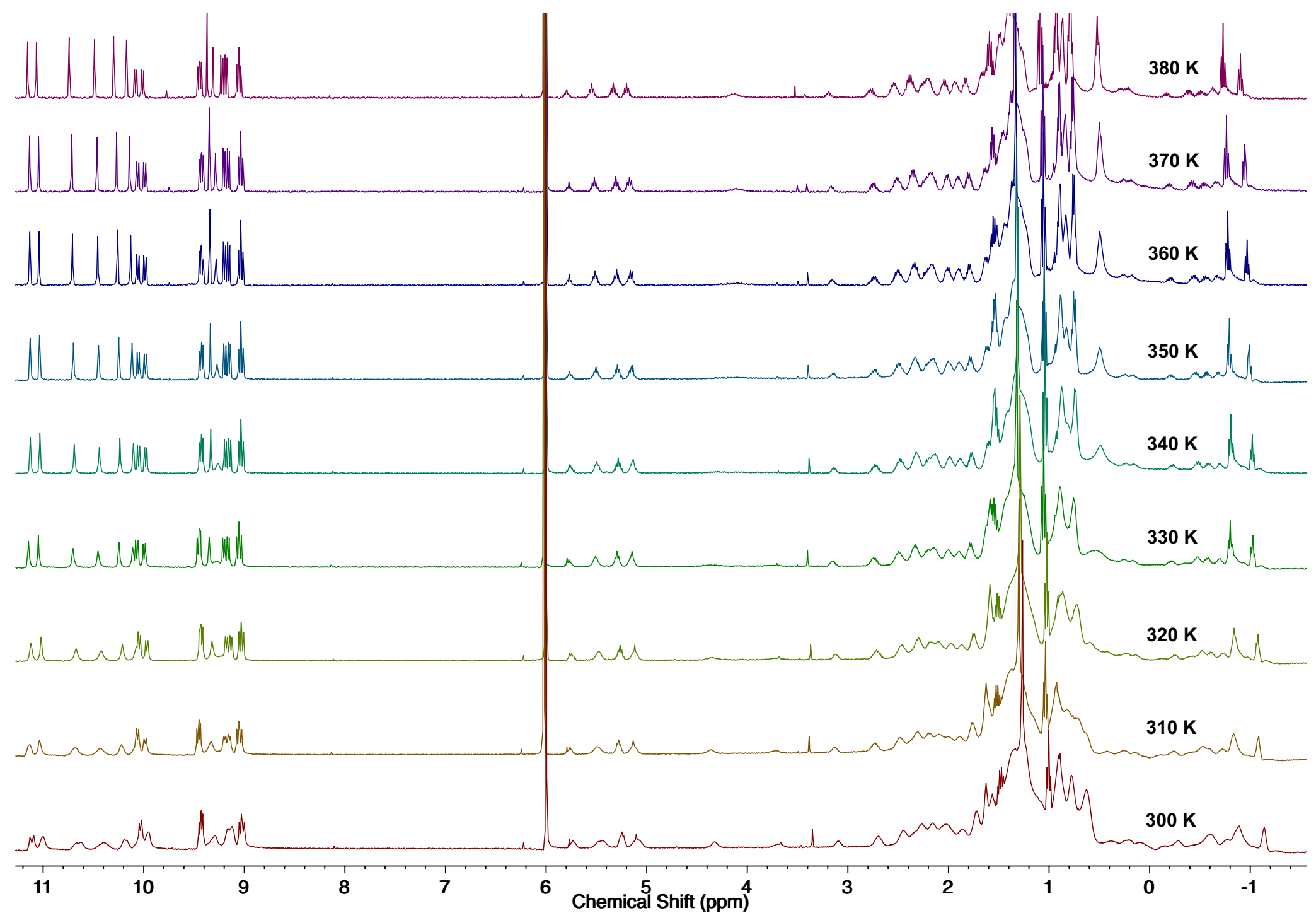

Figure S12. ${ }^{1} \mathrm{H}-\mathrm{NMR}$ spectra of racemic WH[6][6] in $\mathrm{C}_{2} \mathrm{D}_{2} \mathrm{Cl}_{4}$ from $300-380 \mathrm{~K}$ in $10 \mathrm{~K}$ increments. 


\section{DFT Calculations: Structures and Thermochemistry}

\section{a. $\mathrm{hPDI}_{2}$}
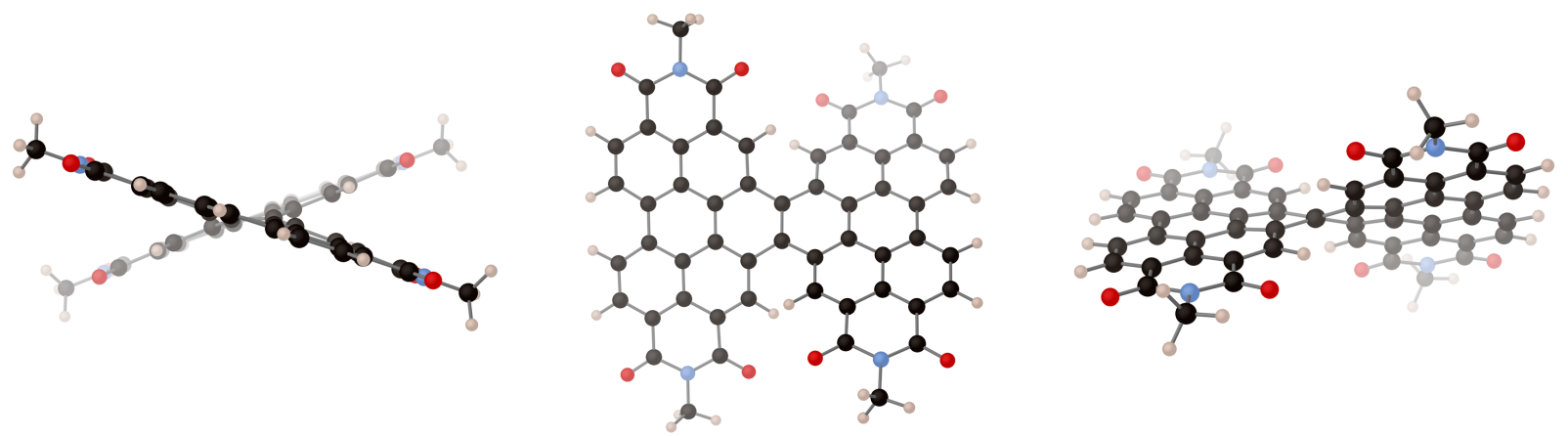

Figure S13. DFT-optimized (B3LYP/6-31G*) structure of chiral-hPDI 2 ( $M$ M $)$ from different perspectives $\left(\Delta \mathrm{G}_{298.15 \mathrm{~K}}=-2892.8018\right.$ hartrees $)$.

Cartesian Coordinates of $\mathbf{M} \boldsymbol{M}-\mathbf{h} \mathbf{P D I}_{\mathbf{2}}$ (from left to right: atom; $\mathrm{x}, \mathrm{y}, \mathrm{z}$ in $\AA$ )

\begin{tabular}{rrrr}
$\mathrm{C}$ & 0.402969 & 2.664167 & -0.126146 \\
$\mathrm{C}$ & 1.591336 & 1.891965 & 0.046453 \\
$\mathrm{C}$ & 2.795341 & 2.538515 & 0.440258 \\
$\mathrm{C}$ & 2.815967 & 3.925786 & 0.676924 \\
$\mathrm{C}$ & 1.652005 & 4.667127 & 0.506862 \\
$\mathrm{C}$ & 0.469733 & 4.045516 & 0.109663 \\
$\mathrm{C}$ & 1.597641 & 0.477356 & -0.169946 \\
$\mathrm{C}$ & 2.807005 & -0.260033 & -0.096043 \\
$\mathrm{C}$ & 3.976734 & 0.417123 & 0.357228 \\
$\mathrm{C}$ & 3.974311 & 1.763450 & 0.624372 \\
$\mathrm{C}$ & -0.825620 & 1.989785 & -0.549889 \\
$\mathrm{C}$ & -0.812840 & 0.571857 & -0.714593 \\
$\mathrm{C}$ & 0.374317 & -0.193284 & -0.484997 \\
$\mathrm{C}$ & -2.024844 & 2.675941 & -0.793632 \\
$\mathrm{C}$ & -3.183076 & 2.011776 & -1.192864 \\
$\mathrm{C}$ & -3.181627 & 0.631602 & -1.357958 \\
$\mathrm{C}$ & -2.002817 & -0.097003 & -1.113072 \\
$\mathrm{C}$ & -1.982772 & -1.508283 & -1.295025 \\
$\mathrm{C}$ & -0.851403 & -2.232314 & -1.012353 \\
$\mathrm{C}$ & 0.343043 & -1.609004 & -0.547453 \\
$\mathrm{C}$ & 5.220962 & 2.402470 & 1.123433 \\
$\mathrm{~N}$ & 5.185137 & 3.789242 & 1.314523 \\
$\mathrm{C}$ & 4.059717 & 4.606655 & 1.117459 \\
$\mathrm{C}$ & -4.416950 & -0.058937 & -1.801993 \\
$\mathrm{~N}$ & -4.333866 & -1.444089 & -2.002851 \\
$\mathrm{C}$ & -3.183190 & -2.223495 & -1.808963 \\
$\mathrm{C}$ & 1.551697 & -2.367029 & -0.286523 \\
$\mathrm{C}$ & 2.792046 & -1.690772 & -0.337425 \\
$\mathrm{O}$ & 4.107825 & 5.813650 & 1.311025 \\
$\mathrm{O}$ & 6.243808 & 1.773332 & 1.354884 \\
$\mathrm{O}$ & -5.469850 & 0.530485 & -2.003016 \\
$\mathrm{O}$ & -3.178352 & -3.423791 & -2.043477 \\
$\mathrm{H}$ & 1.686584 & 5.735020 & 0.692152 \\
$\mathrm{H}$ & -0.413388 & 4.659672 & -0.011940 \\
$\mathrm{H}$ & -2.067693 & 3.751335 & -0.676905 \\
$\mathrm{H}$ & -4.101726 & 2.555358 & -1.383945 \\
& & & \\
\hline
\end{tabular}

$\begin{array}{lrrr}\mathrm{H} & -0.884644 & -3.294478 & -1.211947 \\ \mathrm{C} & 6.432661 & 4.410970 & 1.772557 \\ \mathrm{C} & -5.558766 & -2.104690 & -2.467489 \\ \mathrm{C} & 3.956858 & -3.874756 & -0.501991 \\ \mathrm{C} & 3.984867 & -2.462906 & -0.630676 \\ \mathrm{C} & 5.151924 & -1.865230 & -1.190014 \\ \mathrm{C} & 6.262071 & -2.607215 & -1.506967 \\ \mathrm{C} & 7.433513 & -1.927287 & -2.120609 \\ \mathrm{C} & 6.291425 & -4.009133 & -1.266906 \\ \mathrm{C} & 5.127060 & -4.654564 & -0.766623 \\ \mathrm{C} & 5.146662 & -6.064215 & -0.540703 \\ \mathrm{C} & 6.327108 & -6.765531 & -0.829850 \\ \mathrm{C} & 7.458936 & -6.124770 & -1.330629 \\ \mathrm{C} & 7.451507 & -4.752566 & -1.555167 \\ \mathrm{H} & 6.375513 & -7.835240 & -0.670285 \\ \mathrm{H} & 8.361688 & -6.681419 & -1.556653 \\ \mathrm{C} & 8.662333 & -4.094535 & -2.109020 \\ \mathrm{C} & 2.753957 & -4.526754 & -0.086161 \\ \mathrm{C} & 1.551582 & -3.784033 & 0.022629 \\ \mathrm{C} & 0.410146 & -4.434809 & 0.575472 \\ \mathrm{C} & 0.426772 & -5.766908 & 0.905624 \\ \mathrm{H} & -0.490663 & -3.882447 & 0.805185 \\ \mathrm{C} & -0.787876 & -6.377616 & 1.508024 \\ \mathrm{C} & 2.771160 & -5.929730 & 0.194515 \\ \mathrm{C} & 1.592013 & -6.553830 & 0.687544 \\ \mathrm{C} & 1.583568 & -7.929345 & 0.986336 \\ \mathrm{C} & 2.734136 & -8.682668 & 0.781435 \\ \mathrm{C} & 3.891871 & -8.083911 & 0.288304 \\ \mathrm{C} & 3.945105 & -6.714683 & -0.012572 \\ \mathrm{C} & 0.366927 & -8.584831 & 1.529084 \\ \mathrm{H} & 2.708595 & -9.741212 & 1.015492 \\ \mathrm{H} & 4.766044 & -8.705713 & 0.142332 \\ \mathrm{~N} & 8.566390 & -2.715141 & -2.358381 \\ \mathrm{~N} & -0.742318 & -7.754348 & 1.759393 \\ \mathrm{O} & 9.689190 & -4.714798 & -2.348648 \\ \mathrm{O} & 7.438368 & -0.737842 & -2.405237\end{array}$

$\begin{array}{cccc}\mathrm{O} & 0.327811 & -9.782413 & 1.775974 \\ \mathrm{O} & -1.794008 & -5.734544 & 1.772518 \\ \mathrm{C} & 9.726333 & -2.021566 & -2.929457 \\ \mathrm{C} & -1.960184 & -8.349721 & 2.320406 \\ \mathrm{H} & 4.891576 & -0.122882 & 0.559789 \\ \mathrm{H} & 5.176741 & -0.812646 & -1.436985 \\ \mathrm{H} & 6.259950 & 5.481286 & 1.851205 \\ \mathrm{H} & 6.719595 & 3.996102 & 2.741111 \\ \mathrm{H} & 7.231622 & 4.199701 & 1.059203 \\ \mathrm{H} & -5.346631 & -3.166453 & -2.562987 \\ \mathrm{H} & -6.362759 & -1.934238 & -1.749009 \\ \mathrm{H} & -5.863935 & -1.686554 & -3.429028 \\ \mathrm{H} & 10.530882 & -2.745935 & -3.026868 \\ \mathrm{H} & 9.465769 & -1.603968 & -3.904323 \\ \mathrm{H} & 10.021896 & -1.200455 & -2.273671 \\ \mathrm{H} & -1.781987 & -9.415011 & 2.442934 \\ \mathrm{H} & -2.190326 & -7.885288 & 3.281524 \\ \mathrm{H} & -2.799972 & -8.173939 & 1.645212\end{array}$



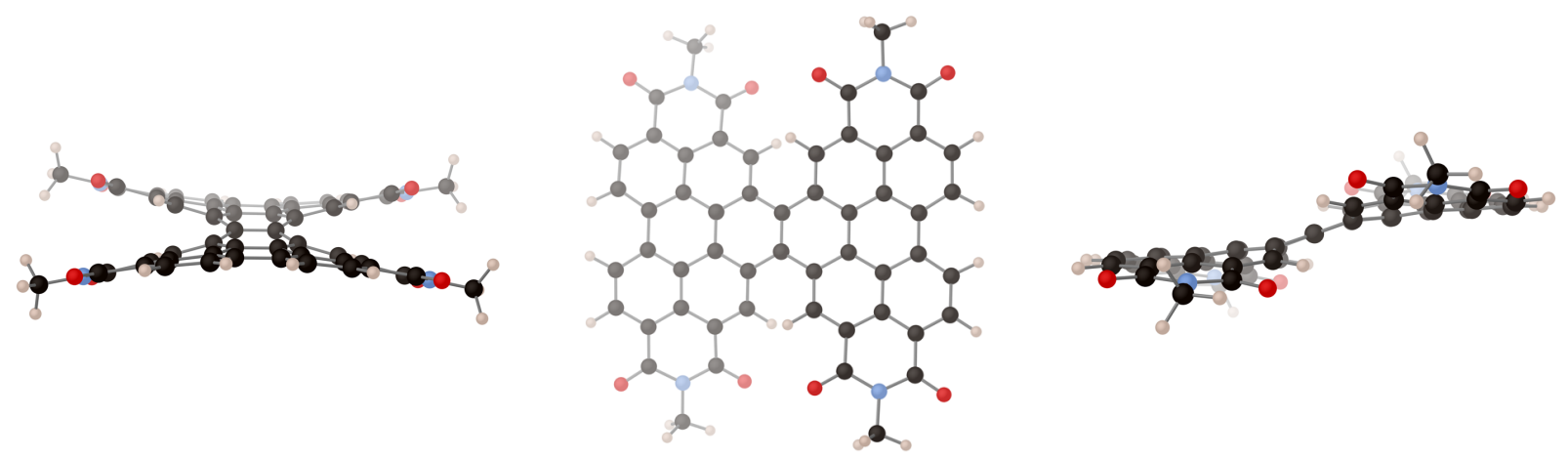

Figure S14. DFT-optimized (B3LYP/6-31G**) structure of meso-hPDI 2 (PM) from different perspectives $\left(\Delta \mathrm{G}_{298.15 \mathrm{~K}}=-2892.7931\right.$ hartrees $)$.

\section{Cartesian Coordinates of $\mathbf{P} \boldsymbol{M}-\mathbf{h} \mathbf{P D I}_{\mathbf{2}}$ (from left to right: atom; x, y, z in $\AA$ )}

$\begin{array}{rrrr}\mathrm{C} & 0.614362 & 2.313866 & -2.440325 \\ \mathrm{C} & 1.721062 & 1.429474 & -2.603966 \\ \mathrm{C} & 2.882613 & 1.886644 & -3.282593 \\ \mathrm{C} & 2.974729 & 3.218348 & -3.725937 \\ \mathrm{C} & 1.922430 & 4.094552 & -3.483521 \\ \mathrm{C} & 0.760428 & 3.644192 & -2.860150 \\ \mathrm{C} & 1.682181 & 0.085868 & -2.110812 \\ \mathrm{C} & 2.816904 & -0.757369 & -2.221796 \\ \mathrm{C} & 3.872307 & -0.320313 & -3.078001 \\ \mathrm{C} & 3.920281 & 0.958767 & -3.573415 \\ \mathrm{C} & -0.641314 & 1.777472 & -1.911714 \\ \mathrm{C} & -0.710376 & 0.390652 & -1.581636 \\ \mathrm{C} & 0.456789 & -0.438728 & -1.594290 \\ \mathrm{C} & -1.806616 & 2.547976 & -1.780512 \\ \mathrm{C} & -3.024557 & 1.981680 & -1.406829 \\ \mathrm{C} & -3.120393 & 0.615046 & -1.168865 \\ \mathrm{C} & -1.965649 & -0.184236 & -1.245615 \\ \mathrm{C} & -2.052452 & -1.591859 & -1.061907 \\ \mathrm{C} & -0.919012 & -2.366187 & -1.061124 \\ \mathrm{C} & 0.383789 & -1.799266 & -1.196123 \\ \mathrm{C} & 5.066629 & 1.366220 & -4.427847 \\ \mathrm{~N} & 5.131915 & 2.718719 & -4.787840 \\ \mathrm{C} & 4.167673 & 3.690392 & -4.472126 \\ \mathrm{C} & -4.437316 & 0.005540 & -0.862856 \\ \mathrm{~N} & -4.480298 & -1.391544 & -0.754861 \\ \mathrm{C} & -3.375994 & -2.249127 & -0.882738 \\ \mathrm{C} & 1.613575 & -2.549983 & -1.001218 \\ \mathrm{C} & 2.832012 & -2.024133 & -1.512019 \\ \mathrm{O} & 4.300742 & 4.856843 & -4.816107 \\ \mathrm{O} & 5.932046 & 0.585654 & -4.797398 \\ \mathrm{O} & -5.458486 & 0.666161 & -0.729727 \\ \mathrm{O} & -3.502306 & -3.464053 & -0.834281 \\ \mathrm{H} & 2.015389 & 5.122246 & -3.816906 \\ \mathrm{H} & -0.052398 & 4.346765 & -2.724882 \\ \mathrm{H} & -1.780389 & 3.609818 & -1.994267 \\ \mathrm{H} & -3.920889 & 2.586831 & -1.325281 \\ \mathrm{C} & -1.060526 & -3.433525 & -0.977464 \\ \mathrm{C} & 6.305706 & 3.123362 & -5.568982 \\ \mathrm{C} & -5.800057 & -1.980370 & -0.502237 \\ \mathrm{C} & 4.002424 & -4.121343 & -0.902733 \\ & 4.064831 & -2.763161 & -1.307019 \\ & 5.821762 & -2.273004 & -1.574977\end{array}$

$\begin{array}{lrrr}\mathrm{C} & 6.433615 & -4.345857 & -1.213376 \\ \mathrm{C} & 5.180271 & -4.935634 & -0.895130 \\ \mathrm{C} & 5.124397 & -6.320865 & -0.559259 \\ \mathrm{C} & 6.303259 & -7.074421 & -0.659200 \\ \mathrm{C} & 7.520168 & -6.493758 & -1.012427 \\ \mathrm{C} & 7.601581 & -5.128217 & -1.262125 \\ \mathrm{H} & 6.288844 & -8.133654 & -0.434756 \\ \mathrm{H} & 8.426436 & -7.086535 & -1.068952 \\ \mathrm{C} & 8.920548 & -4.509218 & -1.547327 \\ \mathrm{C} & 2.775718 & -4.656829 & -0.400672 \\ \mathrm{C} & 1.632308 & -3.822695 & -0.299198 \\ \mathrm{C} & 0.569400 & -4.276604 & 0.539211 \\ \mathrm{C} & 0.521780 & -5.561654 & 1.018351 \\ \mathrm{H} & -0.228966 & -3.614310 & 0.841562 \\ \mathrm{C} & -0.640850 & -5.989056 & 1.840802 \\ \mathrm{C} & 2.742810 & -6.003193 & 0.085990 \\ \mathrm{C} & 1.572392 & -6.477619 & 0.737462 \\ \mathrm{C} & 1.482407 & -7.814657 & 1.164627 \\ \mathrm{C} & 2.548040 & -8.678210 & 0.935969 \\ \mathrm{C} & 3.719632 & -8.210260 & 0.343660 \\ \mathrm{C} & 3.862645 & -6.874611 & -0.060576 \\ \mathrm{C} & 0.275731 & -8.306228 & 1.876041 \\ \mathrm{H} & 2.456840 & -9.710675 & 1.254844 \\ \mathrm{H} & 4.540996 & -8.904613 & 0.217777 \\ \mathrm{~N} & 8.941344 & -3.109625 & -1.667290 \\ \mathrm{~N} & -0.704605 & -7.346979 & 2.180058 \\ \mathrm{O} & 9.942748 & -5.173257 & -1.651336 \\ \mathrm{O} & 7.950031 & -1.059084 & -1.644661 \\ \mathrm{O} & 0.143987 & -9.478098 & 2.201479 \\ \mathrm{O} & -1.521135 & -5.220317 & 2.199805 \\ \mathrm{C} & 10.228033 & -2.443013 & -1.895158 \\ \mathrm{C} & -1.895898 & -7.772029 & 2.923283 \\ \mathrm{H} & 4.661400 & -0.992953 & -3.383860 \\ \mathrm{H} & 5.488577 & -1.111849 & -1.531244 \\ \mathrm{H} & 6.227846 & 4.191665 & -5.754086 \\ \mathrm{H} & 7.215564 & 2.893992 & -5.010683 \\ \mathrm{H} & 6.332502 & 2.570582 & -6.510258 \\ \mathrm{H} & -5.674727 & -3.057975 & -0.435254 \\ \mathrm{H} & -6.208612 & -1.580367 & 0.427806 \\ \mathrm{H} & -6.483106 & -1.723767 & -1.314822 \\ \mathrm{H} & 10.992707 & -3.212633 & -1.961994 \\ \mathrm{H} & 10.183008 & -1.862588 & -2.818877 \\ \mathrm{H} & 10.441105 & -1.759008 & -1.071057 \\ \mathrm{H} & -1.814493 & -8.842234 & 3.095526\end{array}$

H $\quad-1.953225 \quad-7.232909 \quad 3.871079$

H $\quad-2.792159-7.541971 \quad 2.343564$ 

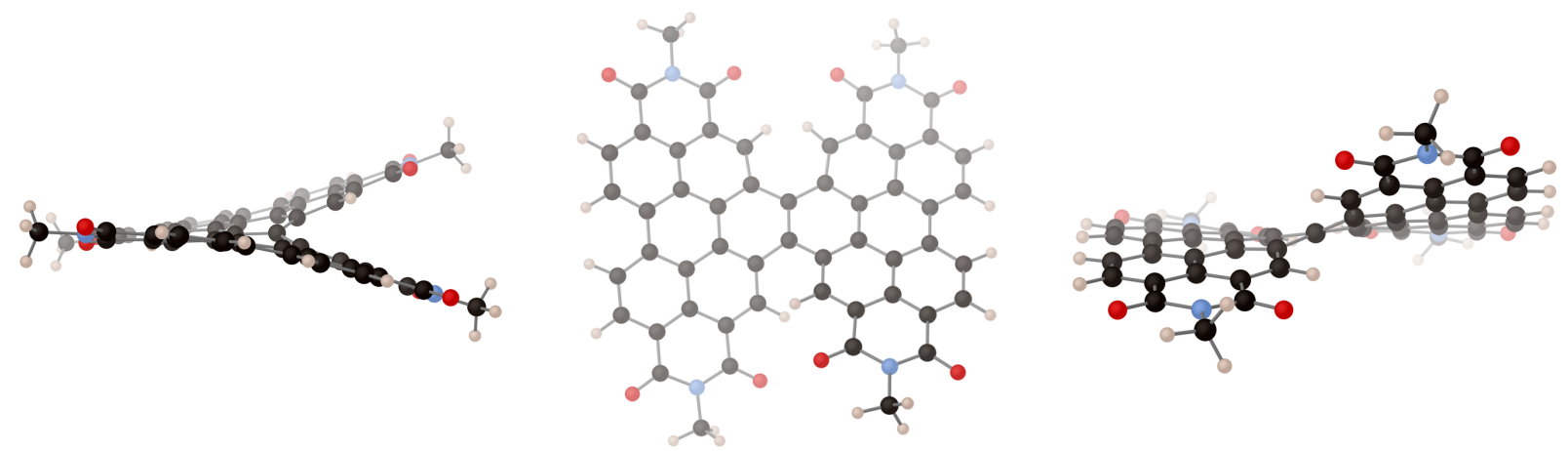

Figure S15. DFT-calculated (B3LYP/6-31G**) helical-to-meso transition-state structure of $\mathbf{h P D I} \mathbf{f r o m}^{*}$ different perspectives $\left(\Delta \mathrm{G}_{298.15 \mathrm{~K}}=-2892.7845\right.$ hartrees $)$.

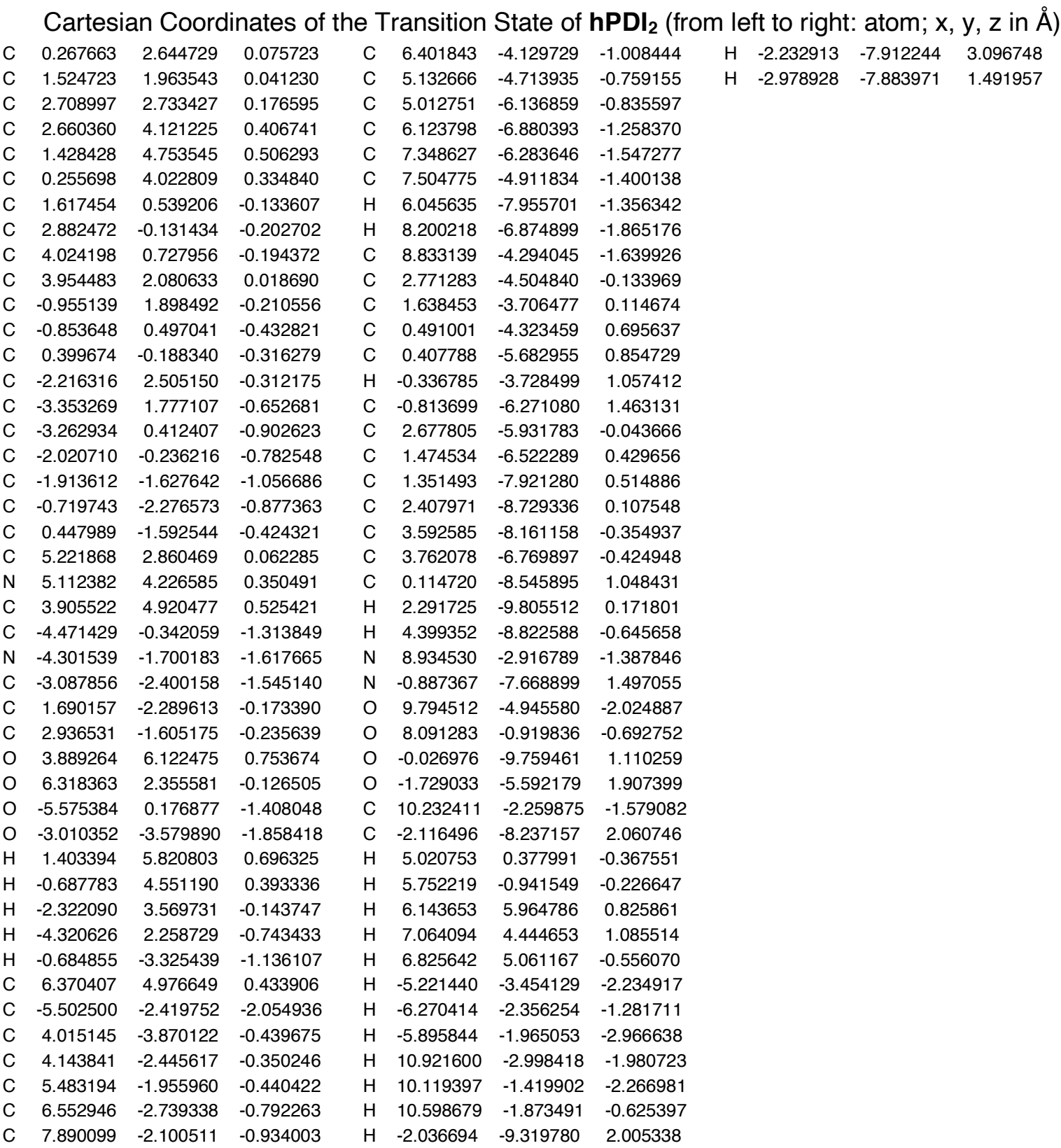


Table S1. Conformational Ensemble of $\mathrm{hPDI}_{2}$ at $298.15 \mathrm{~K}^{\dagger}$

$\begin{array}{ccc}\text { Conformation of } \mathbf{h P D I}_{2} & \Delta \mathrm{G}(\mathrm{kcal} / \mathrm{mol}) & \text { Boltzmann } \\ \text { MM } & 0.0 & \sim 50.0 \\ \boldsymbol{P P} & 0.0 & \sim 50.0 \\ \boldsymbol{P M} & 5.4 & <0.1\end{array}$

${ }^{\dagger}$ As calculated using the Gibbs free energies corresponding to the DFT-optimized structures in Figures S13 and S14.

\section{b. $\mathrm{WH}[6]$}
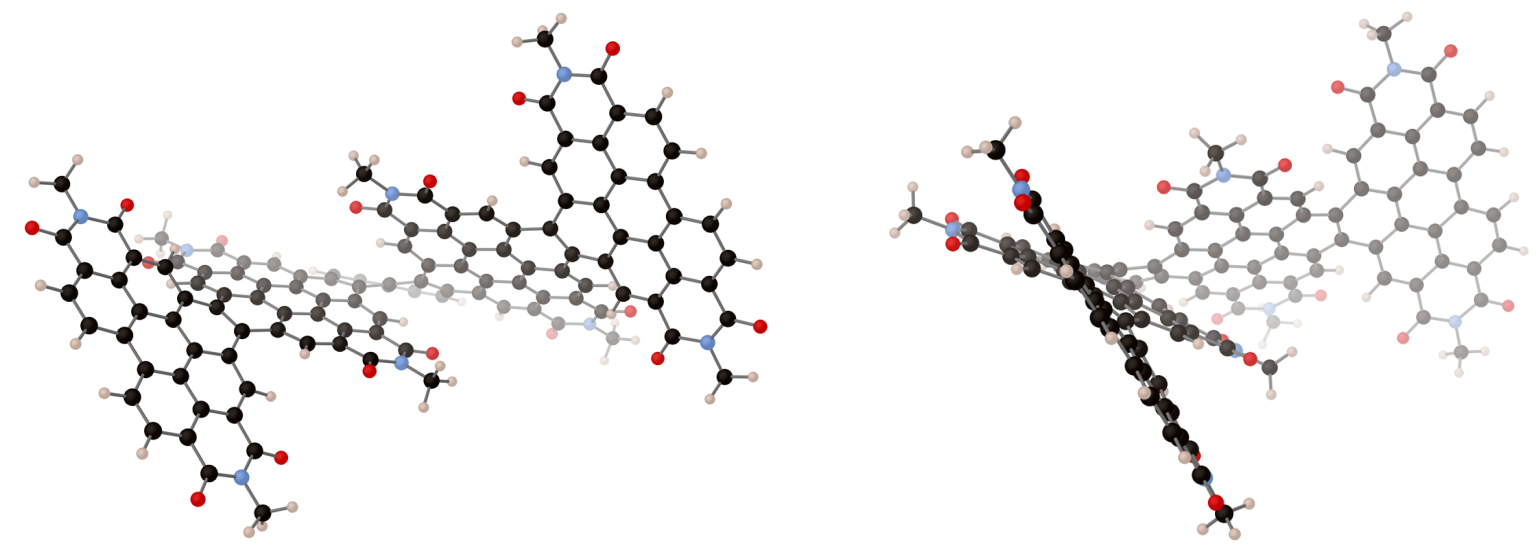

Figure S16. DFT-optimized (B3LYP/6-31G**) structure of $\mathbf{M M - M [ 6 ] - M M}$ from different perspectives $\left(\Delta \mathrm{G}_{298.15 \mathrm{~K}}=-6166.6447\right.$ hartrees $)$.

Cartesian Coordinates of $\mathbf{M M}-\mathbf{M}$ 6]-MM (from left to right: atom; $\mathrm{x}, \mathrm{y}, \mathrm{z}$ in $\AA$ )

\begin{tabular}{|c|c|c|c|c|c|c|c|c|c|c|c|}
\hline C & 0.85840 & 1.21560 & -1.48420 & $\mathrm{H}$ & -7.12000 & 3.56860 & 2.81950 & $\mathrm{H}$ & -7.90430 & 5.53140 & 8.45540 \\
\hline C & -0.40150 & 0.54050 & -1.52480 & $\mathrm{H}$ & -2.69980 & 3.74560 & 4.33700 & $\mathrm{H}$ & -16.75750 & 4.26670 & -3.77880 \\
\hline C & -1.50590 & 1.17680 & -2.13690 & $\mathrm{H}$ & -1.87960 & 2.17530 & 4.62410 & $\mathrm{H}$ & -15.18700 & 4.09030 & -4.61740 \\
\hline C & -1.36960 & 2.49710 & -2.62550 & $\mathrm{H}$ & -1.48370 & 3.12430 & 3.18310 & $\mathrm{H}$ & -15.98650 & 2.65600 & -3.95760 \\
\hline C & -0.14890 & 3.13850 & -2.58490 & $\mathrm{H}$ & -2.88380 & -1.36520 & 1.34730 & & & & \\
\hline C & 1.01560 & 2.49100 & -2.09210 & C & -10.33760 & 1.92130 & 0.82700 & & & & \\
\hline C & -0.55790 & -0.77530 & -0.98820 & C & -9.42970 & 2.38820 & 1.80500 & & & & \\
\hline C & -1.82040 & -1.43400 & -1.03370 & C & -11.93110 & 3.71390 & 1.43970 & & & & \\
\hline C & -2.86950 & -0.78980 & -1.73860 & C & -11.51300 & 2.71110 & 0.53240 & & & & \\
\hline C & -2.73160 & 0.47930 & -2.25690 & C & -12.20130 & 2.59860 & -0.70980 & & & & \\
\hline C & 1.97900 & 0.57880 & -0.86730 & C & -13.30800 & 3.35660 & -0.99000 & & & & \\
\hline C & 1.84490 & -0.75780 & -0.39450 & $\mathrm{H}$ & -11.84000 & 1.94900 & -1.49390 & & & & \\
\hline C & 0.59070 & -1.43580 & -0.45230 & C & -13.81550 & 4.28100 & -0.03670 & & & & \\
\hline C & 3.21930 & 1.26020 & -0.74750 & C & -13.11680 & 4.47310 & 1.18610 & & & & \\
\hline C & 4.34570 & 0.52430 & -0.29470 & C & -13.61500 & 5.40890 & 2.13920 & & & & \\
\hline C & 4.23500 & -0.78230 & 0.12670 & C & -14.78540 & 6.11570 & 1.82410 & & & & \\
\hline C & 2.98070 & -1.43370 & 0.11250 & C & -15.45620 & 5.92910 & 0.61740 & & & & \\
\hline C & 2.86140 & -2.77070 & 0.55590 & C & -14.98010 & 5.01780 & -0.31850 & & & & \\
\hline C & 1.65160 & -3.42060 & 0.48100 & $\mathrm{H}$ & -15.19100 & 6.83250 & 2.52660 & & & & \\
\hline C & 0.49430 & -2.78080 & -0.03170 & $\mathrm{H}$ & -16.35650 & 6.48750 & 0.38550 & & & & \\
\hline C & -3.89630 & 1.11210 & -2.93080 & C & -11.17750 & 3.94880 & 2.62900 & & & & \\
\hline $\mathrm{N}$ & -3.72000 & 2.43980 & -3.35370 & C & -9.92910 & 3.29850 & 2.81690 & & & & \\
\hline C & -2.54780 & 3.19770 & -3.20130 & C & -9.28910 & 3.43890 & 4.08320 & & & & \\
\hline C & 5.45680 & -1.50320 & 0.57790 & C & -9.80450 & 4.24290 & 5.06800 & & & & \\
\hline $\mathrm{N}$ & 5.26480 & -2.80600 & 1.07000 & $\mathrm{H}$ & -8.41410 & 2.85650 & 4.33440 & & & & \\
\hline C & 4.04570 & -3.49440 & 1.08710 & C & -10.99430 & 4.99120 & 4.84900 & & & & \\
\hline $\mathrm{O}$ & -4.95940 & 0.52640 & -3.09040 & C & -11.69360 & 4.84220 & 3.62050 & & & & \\
\hline $\mathrm{O}$ & -2.52240 & 4.36830 & -3.55290 & C & -12.90100 & 5.57270 & 3.40560 & & & & \\
\hline $\mathrm{O}$ & 3.98910 & -4.64070 & 1.51230 & C & -13.35340 & 6.41930 & 4.42800 & & & & \\
\hline $\mathrm{O}$ & 6.56780 & -0.99500 & 0.53960 & C & -12.66780 & 6.54840 & 5.63360 & & & & \\
\hline C & -4.84860 & 3.12680 & -3.99040 & C & -11.49370 & 5.83770 & 5.85570 & & & & \\
\hline
\end{tabular}




\begin{tabular}{|c|c|c|c|c|c|c|c|}
\hline $\mathrm{C}$ & 6.42690 & -3.54020 & 1.58170 & $\mathrm{H}$ & -14.26690 & 6.98540 & 4.29750 \\
\hline C & -1.96770 & -2.77180 & -0.46620 & $\mathrm{H}$ & -13.03690 & 7.19850 & 6.41910 \\
\hline C & -0.77550 & -3.47280 & -0.13360 & C & 3.32860 & 2.62600 & -1.22760 \\
\hline C & -0.80070 & -4.88700 & 0.02460 & C & 2.33130 & 3.11580 & -2.10280 \\
\hline C & -1.94470 & -5.58400 & -0.20310 & C & 3.86000 & 4.96140 & -2.76030 \\
\hline C & -3.17350 & -4.90780 & -0.40920 & C & 2.68920 & 4.19580 & -3.00140 \\
\hline C & -3.23880 & -3.48350 & -0.36290 & C & 1.99080 & 4.41780 & -4.22330 \\
\hline C & -4.33430 & -5.68140 & -0.66990 & C & 2.35850 & 5.40890 & -5.09620 \\
\hline C & -5.53340 & -5.07500 & -0.87420 & $\mathrm{H}$ & 1.18400 & 3.76840 & -4.53170 \\
\hline C & -5.68780 & -3.68090 & -0.62910 & C & 4.21750 & 6.04240 & -3.62800 \\
\hline C & -4.57030 & -2.89930 & -0.22750 & C & 3.44840 & 6.27280 & -4.80050 \\
\hline C & -7.01000 & -3.09530 & -0.71940 & C & 3.78320 & 7.31760 & -5.68120 \\
\hline C & -7.23020 & -1.79290 & -0.21780 & C & 4.86290 & 8.14290 & -5.38670 \\
\hline C & -6.15940 & -1.08010 & 0.40440 & C & 5.62050 & 7.92990 & -4.23720 \\
\hline C & -4.84390 & -1.62450 & 0.43060 & C & 5.33210 & 6.88650 & -3.34410 \\
\hline C & -8.09270 & -3.79150 & -1.31440 & $\mathrm{H}$ & 5.10310 & 8.94660 & -6.07380 \\
\hline C & -9.34580 & -3.23040 & -1.40420 & $\mathrm{H}$ & 6.45550 & 8.59140 & -4.04630 \\
\hline C & -9.58840 & -1.93900 & -0.88150 & C & 4.68930 & 4.65800 & -1.63900 \\
\hline C & -8.53380 & -1.21900 & -0.27210 & C & 4.43180 & 3.49570 & -0.87020 \\
\hline C & -10.88560 & -1.38140 & -0.91660 & C & 5.20230 & 3.29280 & 0.31240 \\
\hline C & -11.11930 & -0.12690 & -0.39930 & C & 6.23660 & 4.12650 & 0.65380 \\
\hline C & -10.08240 & 0.64350 & 0.18870 & $\mathrm{H}$ & 4.96090 & 2.50260 & 1.00860 \\
\hline C & -8.79780 & 0.05420 & 0.30710 & C & 5.79330 & 5.51190 & -1.32210 \\
\hline C & -10.44860 & -4.00340 & -2.03290 & C & 6.58030 & 5.23250 & -0.17170 \\
\hline $\mathrm{N}$ & -11.71250 & -3.39950 & -2.04790 & C & 7.66890 & 6.05740 & 0.16730 \\
\hline C & -12.02430 & -2.14940 & -1.48510 & C & 7.97980 & 7.14750 & -0.63740 \\
\hline O & -13.17020 & -1.72390 & -1.47840 & C & 7.21990 & 7.42440 & -1.77100 \\
\hline O & -10.29030 & -5.11890 & -2.51090 & C & 6.12490 & 6.63060 & -2.14240 \\
\hline C & -12.79320 & -4.17670 & -2.66390 & $\mathrm{H}$ & 8.82200 & 7.77230 & -0.36160 \\
\hline $\mathrm{H}$ & -0.11790 & 4.16170 & -2.93170 & $\mathrm{H}$ & 7.49690 & 8.28020 & -2.37300 \\
\hline $\mathrm{H}$ & -3.81260 & -1.28760 & -1.90340 & C & 1.61210 & 5.55440 & -6.37100 \\
\hline $\mathrm{H}$ & 5.33900 & 0.94790 & -0.31560 & $\mathrm{~N}$ & 1.97690 & 6.62580 & -7.19570 \\
\hline $\mathrm{H}$ & 1.62480 & -4.43640 & 0.84980 & C & 3.01100 & 7.53870 & -6.92920 \\
\hline $\mathrm{H}$ & -4.54630 & 3.49520 & -4.97270 & C & 6.99250 & 3.86280 & 1.90610 \\
\hline $\mathrm{H}$ & -5.66310 & 2.41250 & -4.08170 & $\mathrm{~N}$ & 8.09180 & 4.69090 & 2.16390 \\
\hline $\mathrm{H}$ & -5.15500 & 3.98190 & -3.38390 & C & 8.48510 & 5.78610 & 1.37650 \\
\hline $\mathrm{H}$ & 7.28100 & -2.86820 & 1.55860 & C & -9.11670 & 4.29930 & 6.38450 \\
\hline $\mathrm{H}$ & 6.22500 & -3.87760 & 2.59990 & $\mathrm{~N}$ & -9.64870 & 5.17700 & 7.33660 \\
\hline $\mathrm{H}$ & 6.61720 & -4.41890 & 0.96100 & C & -10.79650 & 5.96710 & 7.15930 \\
\hline $\mathrm{H}$ & 0.11360 & -5.42650 & 0.22920 & C & -13.96650 & 3.20120 & -2.31100 \\
\hline $\mathrm{H}$ & -1.94270 & -6.67000 & -0.20390 & $\mathrm{~N}$ & -15.14570 & 3.92820 & -2.51540 \\
\hline $\mathrm{H}$ & -4.23670 & -6.76060 & -0.73910 & C & -15.70270 & 4.84000 & -1.60340 \\
\hline $\mathrm{H}$ & -6.39340 & -5.67840 & -1.13020 & $\mathrm{O}$ & -16.72630 & 5.45660 & -1.86430 \\
\hline $\mathrm{H}$ & -7.97250 & -4.77860 & -1.73810 & $\mathrm{O}$ & -13.53280 & 2.46800 & -3.18820 \\
\hline $\mathrm{H}$ & -12.14350 & 0.21510 & -0.40200 & $\mathrm{O}$ & -8.13070 & 3.62610 & 6.64940 \\
\hline $\mathrm{H}$ & -12.47360 & -4.51930 & -3.64870 & $\mathrm{O}$ & -11.20110 & 6.70990 & 8.04270 \\
\hline $\mathrm{H}$ & -13.02370 & -5.05350 & -2.05340 & $\mathrm{O}$ & 0.71430 & 4.79540 & -6.70770 \\
\hline $\mathrm{H}$ & -13.66660 & -3.53390 & -2.73730 & $\mathrm{O}$ & 3.26590 & 8.45520 & -7.69810 \\
\hline C & -6.44810 & 0.16330 & 1.04870 & $\mathrm{O}$ & 6.70080 & 2.96420 & 2.68240 \\
\hline C & -7.76320 & 0.72050 & 1.03100 & $\mathrm{O}$ & 9.44720 & 6.47700 & 1.68330 \\
\hline C & -8.05970 & 1.89680 & 1.77240 & C & 1.20890 & 6.77570 & -8.43600 \\
\hline$C$ & -6.97760 & 2.59700 & 2.36710 & C & 8.85740 & 4.38610 & 3.37710 \\
\hline C & -5.69760 & 2.08210 & 2.36680 & C & -8.94350 & 5.24030 & 8.62060 \\
\hline C & -5.42210 & 0.83950 & 1.74820 & C & -15.81650 & 3.72290 & -3.80340 \\
\hline C & -3.86710 & -0.94340 & 1.20290 & $\mathrm{H}$ & 1.59650 & 7.64380 & -8.96320 \\
\hline C & -4.13520 & 0.25530 & 1.82740 & $\mathrm{H}$ & 1.31360 & 5.87600 & -9.04630 \\
\hline C & -3.05570 & 0.93690 & 2.58690 & $\mathrm{H}$ & 0.15080 & 6.90790 & -8.20090 \\
\hline $\mathrm{N}$ & -3.37070 & 2.18220 & 3.14870 & $\mathrm{H}$ & 9.72470 & 5.04100 & 3.39630 \\
\hline$C$ & -4.61040 & 2.83880 & 3.04400 & $\mathrm{H}$ & 8.23800 & 4.54980 & 4.26220 \\
\hline $\mathrm{O}$ & -4.77770 & 3.95930 & 3.50330 & $\mathrm{H}$ & 9.16020 & 3.33770 & 3.36110 \\
\hline O & -1.93120 & 0.46980 & 2.71310 & $\mathrm{H}$ & -9.45380 & 5.97070 & 9.24330 \\
\hline $\mathrm{C}$ & -2.28700 & 2.85360 & 3.87280 & $\mathrm{H}$ & -8.95180 & 4.25710 & 9.09630 \\
\hline
\end{tabular}



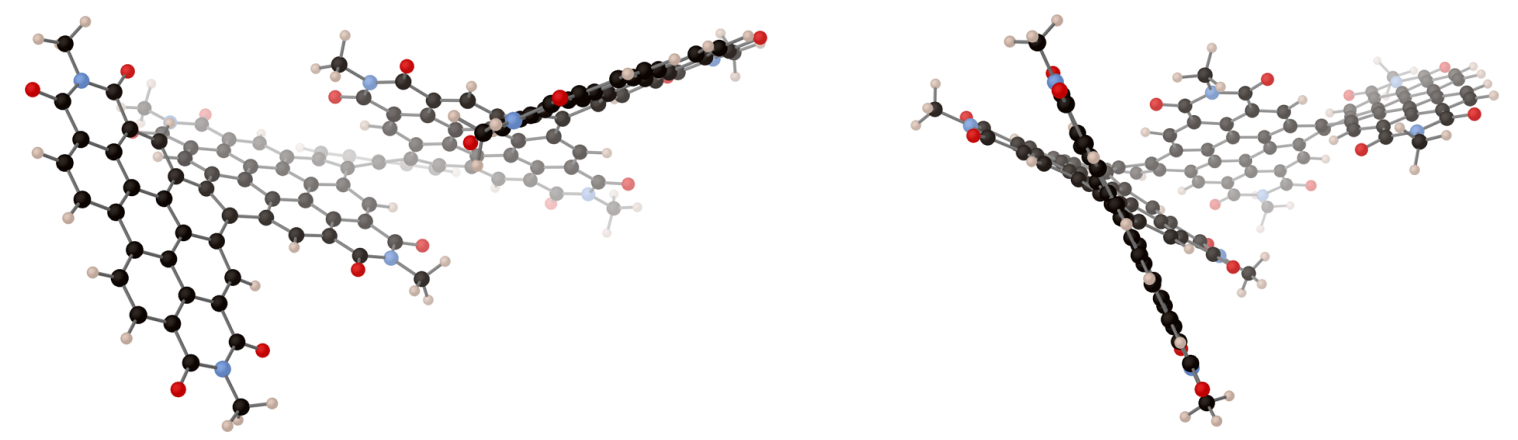

Figure S17. DFT-optimized (B3LYP/6-31G ${ }^{\star *}$ ) structure of $M M-M[6]-P P$ from different perspectives $\left(\Delta \mathrm{G}_{298.15 \mathrm{~K}}=-6166.6490\right.$ hartrees $)$.

Cartesian Coordinates of $\mathbf{M M - M}$ 6]-PP (from left to right: atom; $\mathrm{x}, \mathrm{y}, \mathrm{z}$ in $\AA$ )

\begin{tabular}{|c|c|c|c|c|c|c|c|}
\hline C & 0.78440 & 1.25760 & -1.29380 & C & -10.81310 & 4.69090 & 1.71820 \\
\hline C & -0.46730 & 0.56800 & -1.33160 & C & -9.69520 & 3.92420 & 2.14250 \\
\hline C & -1.57930 & 1.19170 & -1.94190 & C & -9.18600 & 4.16480 & 3.45210 \\
\hline C & -1.43570 & 2.48420 & -2.49860 & C & -9.68800 & 5.15750 & 4.25430 \\
\hline C & -0.23860 & 3.16250 & -2.40740 & $\mathrm{H}$ & -8.42670 & 3.52940 & 3.88400 \\
\hline C & 0.89540 & 2.59320 & -1.77100 & C & -10.73610 & 6.00390 & 3.79860 \\
\hline C & -0.61140 & -0.74650 & -0.78550 & C & -11.31090 & 5.76800 & 2.52030 \\
\hline C & -1.87050 & -1.41240 & -0.81740 & C & -12.37860 & 6.60020 & 2.06800 \\
\hline C & -2.92640 & -0.77980 & -1.52430 & C & -12.82560 & 7.62690 & 2.91380 \\
\hline C & -2.80000 & 0.48410 & -2.05650 & C & -12.26220 & 7.84190 & 4.16940 \\
\hline C & 1.93450 & 0.59400 & -0.76910 & C & -11.22290 & 7.03660 & 4.62150 \\
\hline C & 1.78970 & -0.70050 & -0.19220 & $\mathrm{H}$ & -13.63390 & 8.27450 & 2.59990 \\
\hline C & 0.53570 & -1.38210 & -0.21830 & $\mathrm{H}$ & -12.62430 & 8.63230 & 4.81760 \\
\hline C & 3.21830 & 1.19400 & -0.86880 & C & 3.35350 & 2.47720 & -1.53720 \\
\hline C & 4.30080 & 0.56560 & -0.19900 & C & 2.19190 & 3.25850 & -1.74040 \\
\hline C & 4.15910 & -0.66090 & 0.41310 & C & 3.63100 & 5.22430 & -2.23140 \\
\hline C & 2.91860 & -1.33790 & 0.37470 & C & 2.35490 & 4.68920 & -1.91400 \\
\hline C & 2.79030 & -2.64020 & 0.91110 & C & 1.29920 & 5.60540 & -1.63460 \\
\hline C & 1.59150 & -3.31200 & 0.82700 & C & 1.46490 & 6.96110 & -1.76100 \\
\hline C & 0.44480 & -2.71580 & 0.24200 & $\mathrm{H}$ & 0.35030 & 5.26220 & -1.24770 \\
\hline C & -3.96280 & 1.09150 & -2.75660 & C & 3.80230 & 6.63110 & -2.43530 \\
\hline $\mathrm{N}$ & -3.76520 & 2.37650 & -3.28710 & C & 2.70340 & 7.50320 & -2.20550 \\
\hline C & -2.56910 & 3.10850 & -3.22990 & C & 2.84780 & 8.89220 & -2.37920 \\
\hline C & 5.32710 & -1.26400 & 1.11100 & C & 4.06860 & 9.40910 & -2.79850 \\
\hline $\mathrm{N}$ & 5.14020 & -2.55600 & 1.63300 & C & 5.14890 & 8.56270 & -3.03540 \\
\hline C & 3.95230 & -3.29580 & 1.56640 & C & 5.05270 & 7.17410 & -2.85770 \\
\hline $\mathrm{O}$ & -5.03840 & 0.51600 & -2.86220 & $\mathrm{H}$ & 4.15910 & 10.48170 & -2.92990 \\
\hline 0 & -2.49480 & 4.20630 & -3.76340 & $\mathrm{H}$ & 6.08310 & 9.00470 & -3.35660 \\
\hline 0 & 3.89900 & -4.42180 & 2.04260 & C & 4.75490 & 4.35380 & -2.36240 \\
\hline 0 & 6.39430 & -0.67890 & 1.22460 & C & 4.62640 & 2.98510 & -2.01390 \\
\hline C & -4.87820 & 3.02440 & -3.98850 & C & 5.73170 & 2.12400 & -2.28110 \\
\hline C & 6.26800 & -3.20840 & 2.30710 & C & 6.92290 & 2.60140 & -2.76630 \\
\hline C & -2.00720 & -2.74560 & -0.23600 & $\mathrm{H}$ & 5.64820 & 1.05330 & -2.16240 \\
\hline C & -0.81480 & -3.42770 & 0.12830 & C & 6.00610 & 4.87700 & -2.82300 \\
\hline C & -0.83710 & -4.83970 & 0.31670 & C & 7.10100 & 3.99100 & -3.01660 \\
\hline C & -1.97540 & -5.55230 & 0.09200 & C & 8.33780 & 4.47530 & -3.48340 \\
\hline C & -3.20930 & -4.89190 & -0.14360 & C & 8.49010 & 5.83240 & -3.74880 \\
\hline C & -3.27230 & -3.46650 & -0.12630 & C & 7.42670 & 6.71050 & -3.55180 \\
\hline C & -4.37890 & -5.66050 & -0.39270 & C & 6.17680 & 6.26820 & -3.08990 \\
\hline C & -5.57500 & -5.04970 & -0.62610 & $\mathrm{H}$ & 9.44970 & 6.18590 & -4.10900 \\
\hline C & -5.72040 & -3.64820 & -0.41400 & $\mathrm{H}$ & 7.58500 & 7.75960 & -3.76580 \\
\hline C & -4.59730 & -2.87940 & -0.00520 & C & 0.33820 & 7.86150 & -1.40630 \\
\hline C & -7.01550 & -3.01400 & -0.58090 & $\mathrm{~N}$ & 0.53490 & 9.23090 & -1.62690 \\
\hline C & -7.19610 & -1.67590 & -0.15700 & C & 1.71990 & 9.81610 & -2.10490 \\
\hline C & -6.13190 & -0.98770 & 0.50000 & C & 8.02560 & 1.64420 & -3.03940 \\
\hline C & -4.85020 & -1.58820 & 0.61820 & $\mathrm{~N}$ & 9.24330 & 2.19190 & -3.46350 \\
\hline C & -8.10040 & -3.67830 & -1.20570 & C & 9.48030 & 3.55570 & -3.70760 \\
\hline
\end{tabular}




\begin{tabular}{|c|c|c|c|}
\hline C & -9.30380 & -3.04260 & -1.42140 \\
\hline C & -9.49490 & -1.70390 & -1.00620 \\
\hline C & -8.44580 & -1.01940 & -0.35180 \\
\hline C & -10.73230 & -1.04990 & -1.20670 \\
\hline C & -10.90100 & 0.26320 & -0.82310 \\
\hline C & -9.86130 & 0.99630 & -0.19320 \\
\hline C & -8.65680 & 0.31140 & 0.11130 \\
\hline C & -10.40530 & -3.77630 & -2.09310 \\
\hline $\mathrm{N}$ & -11.61870 & -3.09090 & -2.24680 \\
\hline C & -11.87640 & -1.77680 & -1.81560 \\
\hline $\mathrm{O}$ & -12.98070 & -1.26890 & -1.94670 \\
\hline O & -10.29230 & -4.92750 & -2.49230 \\
\hline C & -12.70280 & -3.83920 & -2.89140 \\
\hline $\mathrm{H}$ & -0.18320 & 4.12080 & -2.90310 \\
\hline $\mathrm{H}$ & -3.86430 & -1.28690 & -1.68690 \\
\hline $\mathrm{H}$ & 5.26170 & 1.05160 & -0.10820 \\
\hline $\mathrm{H}$ & 1.55860 & -4.30550 & 1.25310 \\
\hline $\mathrm{H}$ & -4.61360 & 3.18880 & -5.03540 \\
\hline $\mathrm{H}$ & -5.74340 & 2.37060 & -3.91410 \\
\hline $\mathrm{H}$ & -5.08390 & 3.99430 & -3.53160 \\
\hline $\mathrm{H}$ & 7.11280 & -2.52490 & 2.27760 \\
\hline $\mathrm{H}$ & 5.99770 & -3.44110 & 3.33930 \\
\hline $\mathrm{H}$ & 6.51100 & -4.14320 & 1.79780 \\
\hline $\mathrm{H}$ & 0.08000 & -5.36680 & 0.54360 \\
\hline $\mathrm{H}$ & -1.96210 & -6.63800 & 0.11600 \\
\hline $\mathrm{H}$ & -4.29050 & -6.74190 & -0.43540 \\
\hline $\mathrm{H}$ & -6.43700 & -5.65020 & -0.88480 \\
\hline $\mathrm{H}$ & -8.01380 & -4.69360 & -1.56770 \\
\hline $\mathrm{H}$ & -11.88440 & 0.68930 & -0.96070 \\
\hline $\mathrm{H}$ & -12.37900 & -4.17600 & -3.87800 \\
\hline $\mathrm{H}$ & -12.95470 & -4.71880 & -2.29470 \\
\hline $\mathrm{H}$ & -13.56090 & -3.17680 & -2.97230 \\
\hline C & -6.38600 & 0.30080 & 1.06090 \\
\hline C & -7.64600 & 0.94870 & 0.89140 \\
\hline C & -7.90690 & 2.20430 & 1.50600 \\
\hline C & -6.83000 & 2.84760 & 2.17020 \\
\hline C & -5.60430 & 2.23510 & 2.32570 \\
\hline C & -5.36920 & 0.94410 & 1.79960 \\
\hline C & -3.88350 & -0.93680 & 1.42580 \\
\hline C & -4.12900 & 0.29060 & 1.99890 \\
\hline C & -3.07150 & 0.93680 & 2.81430 \\
\hline$N$ & -3.34710 & 2.22490 & 3.29530 \\
\hline C & -4.53210 & 2.94980 & 3.06590 \\
\hline $\mathrm{O}$ & -4.66830 & 4.09320 & 3.47540 \\
\hline $\mathrm{O}$ & -1.99510 & 0.40590 & 3.05560 \\
\hline C & -2.28430 & 2.85720 & 4.08340 \\
\hline $\mathrm{H}$ & -6.92110 & 3.85590 & 2.54650 \\
\hline $\mathrm{H}$ & -2.64430 & 3.83100 & 4.40610 \\
\hline $\mathrm{H}$ & -2.04330 & 2.22930 & 4.94360 \\
\hline $\mathrm{H}$ & -1.38290 & 2.96590 & 3.47610 \\
\hline $\mathrm{H}$ & -2.92880 & -1.39960 & 1.62990 \\
\hline C & -10.04560 & 2.35710 & 0.28240 \\
\hline C & -9.20730 & 2.83520 & 1.31690 \\
\hline C & -11.44640 & 4.39370 & 0.47390 \\
\hline C & -11.06360 & 3.23780 & -0.25060 \\
\hline C & -11.60790 & 3.05310 & -1.55550 \\
\hline C & -12.55850 & 3.89850 & -2.06770 \\
\hline $\mathrm{H}$ & -11.24120 & 2.27300 & -2.20740 \\
\hline C & -13.04940 & 4.98900 & -1.29630 \\
\hline C & -12.48160 & 5.25000 & -0.01930 \\
\hline C & -12.95870 & 6.35340 & 0.74850 \\
\hline C & -13.98020 & 7.14720 & 0.20590 \\
\hline C & -14.52440 & 6.88830 & -1.04960 \\
\hline C & -14.06520 & 5.81730 & -1.80850 \\
\hline $\mathrm{H}$ & -14.36590 & 7.99010 & 0.76500 \\
\hline $\mathrm{H}$ & -15.31030 & 7.51400 & -1.45800 \\
\hline
\end{tabular}

$\begin{array}{cccc}\mathrm{C} & -9.13500 & 5.32170 & 5.62350 \\ \mathrm{~N} & -9.64360 & 6.38190 & 6.38510 \\ \mathrm{C} & -10.65770 & 7.26430 & 5.97450 \\ \mathrm{C} & -13.06330 & 3.66710 & -3.44500 \\ \mathrm{~N} & -14.09990 & 4.50170 & -3.88270 \\ \mathrm{C} & -14.64750 & 5.56900 & -3.15140 \\ \mathrm{O} & -15.54900 & 6.25880 & -3.60840 \\ \mathrm{O} & -12.62580 & 2.79160 & -4.17860 \\ \mathrm{O} & -8.27330 & 4.58810 & 6.08780 \\ \mathrm{O} & -11.05480 & 8.16180 & 6.70530 \\ \mathrm{O} & -0.72280 & 7.45670 & -0.95170 \\ \mathrm{O} & 1.81170 & 11.02510 & -2.26970 \\ \mathrm{O} & 7.91070 & 0.43470 & -2.89720 \\ \mathrm{O} & 10.57210 & 3.95320 & -4.09150 \\ \mathrm{C} & -0.59820 & 10.10410 & -1.30410 \\ \mathrm{C} & 10.33620 & 1.23890 & -3.68470 \\ \mathrm{C} & -9.06610 & 6.54810 & 7.72310 \\ \mathrm{C} & -14.62440 & 4.22900 & -5.22460 \\ \mathrm{H} & -0.31310 & 11.12330 & -1.55250 \\ \mathrm{H} & -1.47420 & 9.79650 & -1.87840 \\ \mathrm{H} & -0.83930 & 10.02100 & -0.24200 \\ \mathrm{H} & 11.21830 & 1.80580 & -3.97150 \\ \mathrm{H} & 10.51990 & 0.67340 & -2.76900 \\ \mathrm{H} & 10.06120 & 0.53370 & -4.47220 \\ \mathrm{H} & -9.53300 & 7.41650 & 8.18110 \\ \mathrm{H} & -9.25140 & 5.65320 & 8.32130 \\ \mathrm{H} & -7.98610 & 6.68710 & 7.64250 \\ \mathrm{H} & -15.45280 & 4.91030 & -5.40160 \\ \mathrm{H} & -13.83950 & 4.37970 & -5.96920 \\ \mathrm{H} & -14.95650 & 3.19080 & -5.28300 \\ & & & \end{array}$



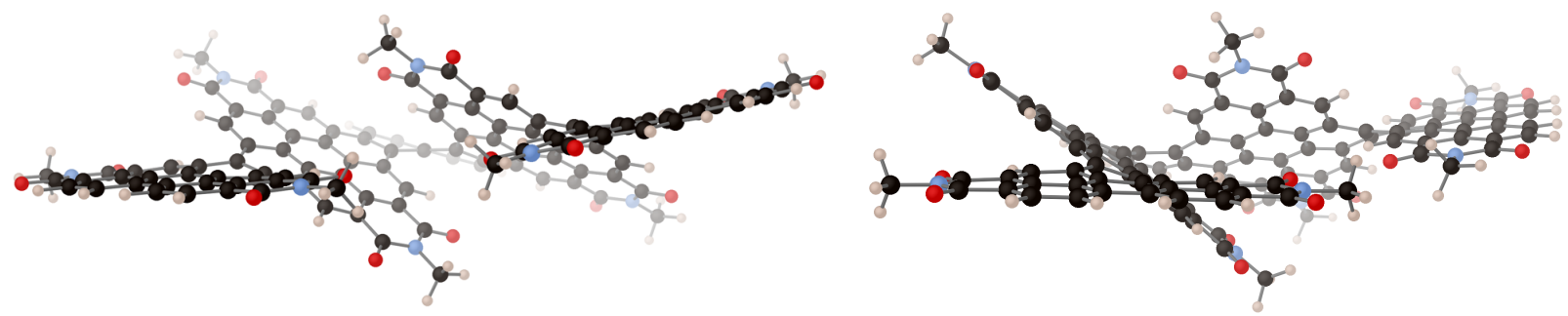

Figure S18. DFT-optimized (B3LYP/6-31G*) structure of PP-M[6]-PP from different perspectives $\left(\Delta \mathrm{G}_{298.15 \mathrm{~K}}=-6166.6475\right.$ hartrees $)$.

Cartesian Coordinates of PP-M[6]-PP (from left to right: atom; x, y, z in $\AA$ )

\begin{tabular}{|c|c|c|c|}
\hline C & 0.97730 & 1.25290 & -1.54500 \\
\hline C & -0.25780 & 0.58640 & -1.80800 \\
\hline C & -1.22160 & 1.21150 & -2.63100 \\
\hline C & -0.93230 & 2.46920 & -3.20730 \\
\hline C & 0.23190 & 3.13560 & -2.89110 \\
\hline C & 1.19200 & 2.57900 & -2.00600 \\
\hline C & -0.53450 & -0.70200 & -1.26020 \\
\hline C & -1.80240 & -1.31240 & -1.45960 \\
\hline C & -2.71460 & -0.67650 & -2.34080 \\
\hline C & -2.43580 & 0.54240 & -2.91840 \\
\hline C & 1.99670 & 0.58170 & -0.80430 \\
\hline C & 1.73210 & -0.71530 & -0.27600 \\
\hline C & 0.48500 & -1.36590 & -0.51160 \\
\hline C & 3.27640 & 1.17780 & -0.64100 \\
\hline C & 4.20650 & 0.52280 & 0.21000 \\
\hline C & 3.94940 & -0.71970 & 0.74860 \\
\hline C & 2.73120 & -1.38140 & 0.47140 \\
\hline C & 2.48930 & -2.68810 & 0.95560 \\
\hline C & 1.30440 & -3.33050 & 0.67270 \\
\hline C & 0.27890 & -2.69360 & -0.06940 \\
\hline C & -3.42670 & 1.14640 & -3.84870 \\
\hline $\mathrm{N}$ & -3.06630 & 2.37460 & -4.42650 \\
\hline C & -1.87090 & 3.07170 & -4.18650 \\
\hline C & 4.96490 & -1.35200 & 1.63330 \\
\hline $\mathrm{N}$ & 4.67540 & -2.65380 & 2.07970 \\
\hline $\mathrm{C}$ & 3.50920 & -3.37460 & 1.78740 \\
\hline $\mathrm{O}$ & -4.49610 & 0.60840 & -4.10520 \\
\hline $\mathrm{O}$ & -1.64320 & 4.12090 & -4.77100 \\
\hline $\mathrm{O}$ & 3.36030 & -4.50880 & 2.22200 \\
\hline $\mathrm{O}$ & 5.99750 & -0.78250 & 1.95510 \\
\hline C & -3.99350 & 3.00230 & -5.37350 \\
\hline C & 5.65710 & -3.33510 & 2.93030 \\
\hline C & -2.08540 & -2.60200 & -0.84460 \\
\hline C & -0.98920 & -3.34600 & -0.33430 \\
\hline C & -1.12650 & -4.74890 & -0.12480 \\
\hline C & -2.28560 & -5.38310 & -0.45730 \\
\hline C & -3.44300 & -4.63960 & -0.81250 \\
\hline C & -3.40530 & -3.21640 & -0.82550 \\
\hline C & -4.63980 & -5.32320 & -1.15230 \\
\hline C & -5.77570 & -4.63410 & -1.45010 \\
\hline C & -5.84530 & -3.22700 & -1.23430 \\
\hline C & -4.68740 & -2.52550 & -0.79880 \\
\hline C & -7.11120 & -2.53570 & -1.39790 \\
\hline C & -7.22690 & -1.18520 & -0.99040 \\
\hline C & -6.11250 & -0.52550 & -0.38590 \\
\hline C & -4.86730 & -1.19400 & -0.23360 \\
\hline C & -8.24550 & -3.16960 & -1.96780 \\
\hline$C$ & -9.44820 & -2.51420 & -2.10590 \\
\hline C & -9.59070 & -1.18010 & -1.65910 \\
\hline C & -8.47760 & -0.50540 & -1.10380 \\
\hline & -10.82570 & -0.50390 & -1.78660 \\
\hline
\end{tabular}

\begin{tabular}{|c|c|c|c|}
\hline C & -7.93670 & 7.21390 & -0.48170 \\
\hline $\mathrm{H}$ & -6.86390 & 5.41370 & -0.57320 \\
\hline C & -9.18360 & 7.86630 & -0.26910 \\
\hline C & -10.34170 & 7.08590 & -0.00000 \\
\hline C & -11.59720 & 7.73430 & 0.20350 \\
\hline C & -11.64030 & 9.13440 & 0.11940 \\
\hline C & -10.50250 & 9.88900 & -0.16030 \\
\hline C & -9.27410 & 9.26790 & -0.35880 \\
\hline $\mathrm{H}$ & -12.57640 & 9.65780 & 0.26640 \\
\hline $\mathrm{H}$ & -10.55330 & 10.96990 & -0.23240 \\
\hline C & 3.53780 & 2.47810 & -1.23900 \\
\hline C & 2.44110 & 3.25200 & -1.68540 \\
\hline C & 3.91110 & 5.24870 & -1.80560 \\
\hline C & 2.60910 & 4.68620 & -1.80450 \\
\hline C & 1.49570 & 5.57630 & -1.79070 \\
\hline C & 1.65420 & 6.93470 & -1.88450 \\
\hline $\mathrm{H}$ & 0.48920 & 5.20990 & -1.64900 \\
\hline C & 4.09380 & 6.66090 & -1.95290 \\
\hline$C$ & 2.95120 & 7.50660 & -2.00600 \\
\hline C & 3.09960 & 8.89900 & -2.14540 \\
\hline C & 4.37450 & 9.44480 & -2.24400 \\
\hline C & 5.50040 & 8.62570 & -2.20030 \\
\hline C & 5.39700 & 7.23400 & -2.05310 \\
\hline $\mathrm{H}$ & 4.46980 & 10.51860 & -2.35380 \\
\hline $\mathrm{H}$ & 6.47380 & 9.09170 & -2.28220 \\
\hline C & 5.05110 & 4.39760 & -1.68560 \\
\hline C & 4.87420 & 3.01700 & -1.41020 \\
\hline C & 6.02710 & 2.17860 & -1.45060 \\
\hline C & 7.28780 & 2.68600 & -1.63620 \\
\hline $\mathrm{H}$ & 5.93950 & 1.10360 & -1.39330 \\
\hline C & 6.36430 & 4.95100 & -1.83020 \\
\hline C & 7.49200 & 4.08560 & -1.78920 \\
\hline C & 8.79450 & 4.59890 & -1.93610 \\
\hline C & 8.97710 & 5.96710 & -2.10920 \\
\hline C & 7.87930 & 6.82450 & -2.14450 \\
\hline C & 6.56380 & 6.35200 & -2.01430 \\
\hline $\mathrm{H}$ & 9.98710 & 6.34630 & -2.21800 \\
\hline $\mathrm{H}$ & 8.06300 & 7.88330 & -2.28100 \\
\hline C & 0.44960 & 7.80380 & -1.85010 \\
\hline $\mathrm{N}$ & 0.66130 & 9.17770 & -2.02560 \\
\hline C & 1.91420 & 9.79380 & -2.17940 \\
\hline C & 8.43950 & 1.74940 & -1.68670 \\
\hline $\mathrm{N}$ & 9.71240 & 2.32370 & -1.79760 \\
\hline C & 9.97510 & 3.69850 & -1.92160 \\
\hline C & -6.73520 & 7.99910 & -0.86190 \\
\hline$N$ & -6.88420 & 9.39160 & -0.91310 \\
\hline C & -8.08000 & 10.09060 & -0.67990 \\
\hline C & -14.80840 & 2.41730 & 1.14220 \\
\hline $\mathrm{N}$ & -16.02150 & 3.06950 & 1.39990 \\
\hline C & -16.22170 & 4.46020 & 1.36010 \\
\hline $\mathrm{O}$ & -17.31800 & 4.95230 & 1.59060 \\
\hline
\end{tabular}




\begin{tabular}{|c|c|c|c|}
\hline C & -10.97220 & 0.77930 & -1.30580 \\
\hline C & -9.90340 & 1.46680 & -0.67430 \\
\hline C & -8.63080 & 0.83460 & -0.64500 \\
\hline C & -10.59700 & -3.21930 & -2.73240 \\
\hline$N$ & -11.78710 & -2.49270 & -2.86590 \\
\hline C & -11.98310 & -1.16420 & -2.44950 \\
\hline $\mathrm{O}$ & -13.05000 & -0.59340 & -2.62270 \\
\hline O & -10.53260 & -4.37580 & -3.12730 \\
\hline C & -12.90510 & -3.19960 & -3.50010 \\
\hline $\mathrm{H}$ & 0.41130 & 4.07320 & -3.39710 \\
\hline $\mathrm{H}$ & -3.64800 & -1.15000 & -2.60710 \\
\hline $\mathrm{H}$ & 5.13360 & 0.99610 & 0.49850 \\
\hline $\mathrm{H}$ & 1.17960 & -4.32630 & 1.07590 \\
\hline $\mathrm{H}$ & -3.51550 & 3.09970 & -6.35060 \\
\hline $\mathrm{H}$ & -4.87610 & 2.37110 & -5.44160 \\
\hline $\mathrm{H}$ & -4.25860 & 4.00130 & -5.02130 \\
\hline $\mathrm{H}$ & 6.50120 & -2.66370 & 3.06600 \\
\hline $\mathrm{H}$ & 5.20460 & -3.58230 & 3.89300 \\
\hline $\mathrm{H}$ & 5.97610 & -4.26410 & 2.45340 \\
\hline $\mathrm{H}$ & -0.27790 & -5.33080 & 0.21000 \\
\hline $\mathrm{H}$ & -2.35560 & -6.46620 & -0.41670 \\
\hline $\mathrm{H}$ & -4.62410 & -6.40840 & -1.19690 \\
\hline $\mathrm{H}$ & -6.65570 & -5.18010 & -1.75930 \\
\hline $\mathrm{H}$ & -8.20260 & -4.18450 & -2.33840 \\
\hline $\mathrm{H}$ & -11.92600 & 1.25740 & -1.47510 \\
\hline $\mathrm{H}$ & -12.61700 & -3.52270 & -4.50260 \\
\hline $\mathrm{H}$ & -13.16020 & -4.08600 & -2.91550 \\
\hline $\mathrm{H}$ & -13.74820 & -2.51490 & -3.54470 \\
\hline C & -6.27550 & 0.81330 & 0.08610 \\
\hline C & -7.50010 & 1.51870 & -0.10500 \\
\hline C & -7.60050 & 2.89310 & 0.23920 \\
\hline C & -6.52010 & 3.48540 & 0.94400 \\
\hline C & -5.36870 & 2.77850 & 1.22490 \\
\hline C & -5.21050 & 1.45060 & 0.76320 \\
\hline C & -3.87490 & -0.56110 & 0.56140 \\
\hline C & -4.02850 & 0.72170 & 1.03750 \\
\hline C & -2.94070 & 1.33890 & 1.83870 \\
\hline$N$ & -3.16240 & 2.64250 & 2.30260 \\
\hline C & -4.31360 & 3.41460 & 2.05870 \\
\hline O & -4.43340 & 4.54110 & 2.51820 \\
\hline O & -1.89110 & 0.76280 & 2.09560 \\
\hline C & -2.08890 & 3.22860 & 3.11130 \\
\hline $\mathrm{H}$ & -6.59680 & 4.48490 & 1.34880 \\
\hline $\mathrm{H}$ & -2.40160 & 4.22790 & 3.40380 \\
\hline $\mathrm{H}$ & -1.90920 & 2.60840 & 3.99220 \\
\hline $\mathrm{H}$ & -1.16650 & 3.27010 & 2.52820 \\
\hline $\mathrm{H}$ & -2.96830 & -1.08060 & 0.83120 \\
\hline C & -10.03060 & 2.83050 & -0.18640 \\
\hline C & -8.85490 & 3.59210 & 0.00450 \\
\hline C & -11.39480 & 4.86280 & 0.22370 \\
\hline C & -11.30700 & 3.45170 & 0.11150 \\
\hline C & -12.46290 & 2.68370 & 0.43880 \\
\hline C & -13.65720 & 3.27570 & 0.76310 \\
\hline $\mathrm{H}$ & -12.41700 & 1.60610 & 0.50690 \\
\hline C & -13.79100 & 4.69240 & 0.75620 \\
\hline C & -12.65020 & 5.49740 & 0.49030 \\
\hline C & -12.77710 & 6.91860 & 0.49720 \\
\hline C & -14.03520 & 7.47360 & 0.77610 \\
\hline C & -15.14410 & 6.67540 & 1.04760 \\
\hline C & -15.03250 & 5.28930 & 1.04390 \\
\hline $\mathrm{H}$ & -14.16290 & 8.54830 & 0.79020 \\
\hline $\mathrm{H}$ & -16.10960 & 7.11630 & 1.27000 \\
\hline C & -10.22390 & 5.66090 & 0.05230 \\
\hline C & -8.95480 & 5.03600 & -0.05030 \\
\hline C & -7.82890 & 5.85230 & -0.36040 \\
\hline
\end{tabular}

$\begin{array}{cccc}\text { O } & -14.73360 & 1.19930 & 1.22700 \\ \text { O } & -5.65530 & 7.48520 & -1.11620 \\ \text { O } & -8.12930 & 11.31130 & -0.75130 \\ \text { O } & -0.68190 & 7.36920 & -1.69150 \\ \text { O } & 2.01570 & 11.00470 & -2.32390 \\ \text { O } & 8.31510 & 0.53420 & -1.62320 \\ \text { O } & 11.11870 & 4.12210 & -2.02150 \\ \text { C } & -0.54080 & 10.01730 & -2.03100 \\ \mathrm{C} & 10.84320 & 1.38980 & -1.79900 \\ \mathrm{C} & -5.68120 & 10.15390 & -1.26210 \\ \mathrm{C} & -17.15630 & 2.20640 & 1.74430 \\ \mathrm{H} & -0.22730 & 11.04470 & -2.19950 \\ \mathrm{H} & -1.21720 & 9.68420 & -2.82070 \\ \mathrm{H} & -1.06090 & 9.92540 & -1.07500 \\ \mathrm{H} & 11.75680 & 1.97510 & -1.86610 \\ \mathrm{H} & 10.83040 & 0.79660 & -0.88240 \\ \mathrm{H} & 10.76070 & 0.70820 & -2.64840 \\ \mathrm{H} & -5.93830 & 11.21000 & -1.23550 \\ \mathrm{H} & -4.88560 & 9.93020 & -0.54870 \\ \mathrm{H} & -5.33680 & 9.86700 & -2.25810 \\ \mathrm{H} & -18.01480 & 2.84620 & 1.93240 \\ \mathrm{H} & -17.36190 & 1.51920 & 0.92080 \\ \mathrm{H} & -16.91400 & 1.61530 & 2.62990 \\ & & & \end{array}$



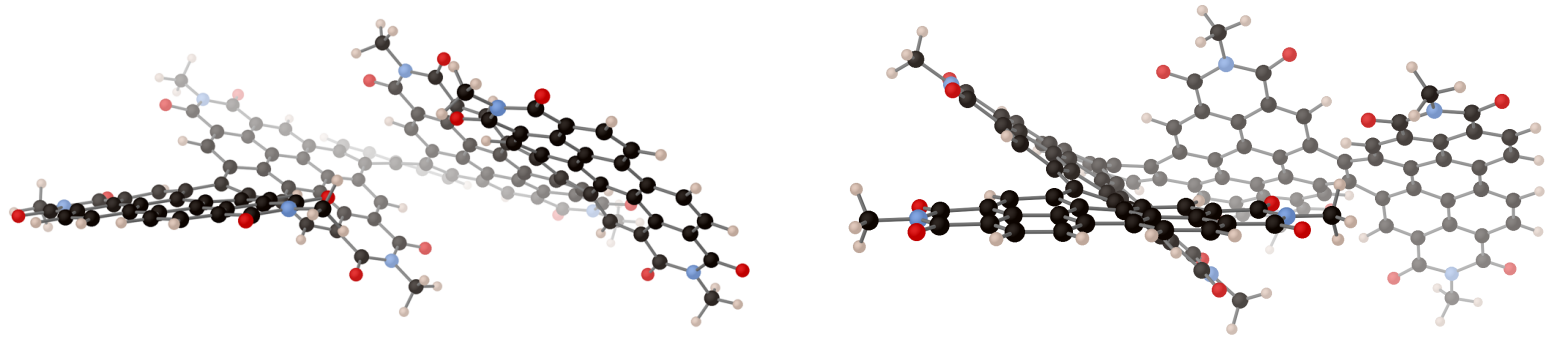

Figure S19. DFT-optimized (B3LYP/6-31G**) structure of $P \boldsymbol{P}-\mathbf{M}[6]-P \boldsymbol{M}$ from different perspectives $\left(\Delta \mathrm{G}_{298.15 \mathrm{~K}}=-6166.6401\right.$ hartrees $)$.

Cartesian Coordinates of PP-M[6]-PM (from left to right: atom; $\mathrm{x}, \mathrm{y}, \mathrm{z}$ in $\AA$ )

\begin{tabular}{|c|c|c|c|c|c|c|c|}
\hline C & 0.92710 & 1.20980 & -1.55180 & C & -7.82870 & 7.14590 & 0.42030 \\
\hline C & -0.30230 & 0.51360 & -1.74460 & $\mathrm{H}$ & -6.70200 & 5.39170 & 0.21440 \\
\hline C & -1.28980 & 1.07540 & -2.58520 & C & -9.08240 & 7.80040 & 0.26380 \\
\hline C & -1.04050 & 2.30730 & -3.23320 & C & -10.15840 & 7.09170 & -0.33440 \\
\hline C & 0.11350 & 3.01330 & -2.97130 & C & -11.37320 & 7.77990 & -0.62950 \\
\hline C & 1.10330 & 2.51370 & -2.08480 & C & -11.50140 & 9.11010 & -0.20290 \\
\hline C & -0.54920 & -0.73980 & -1.10730 & C & -10.45290 & 9.78500 & 0.41930 \\
\hline C & -1.81050 & -1.37990 & -1.24620 & C & -9.23200 & 9.15130 & 0.62730 \\
\hline C & -2.73390 & -0.82630 & -2.17070 & $\mathrm{H}$ & -12.42360 & 9.64930 & -0.38230 \\
\hline C & -2.48810 & 0.36130 & -2.82270 & $\mathrm{H}$ & -10.55730 & 10.82090 & 0.72290 \\
\hline C & 1.97990 & 0.59370 & -0.80850 & C & 3.47330 & 2.48780 & -1.39360 \\
\hline C & 1.74620 & -0.67050 & -0.19270 & C & 2.34470 & 3.22080 & -1.82730 \\
\hline C & 0.49590 & -1.34410 & -0.34290 & C & 3.75050 & 5.24800 & -2.06270 \\
\hline C & 3.25920 & 1.21130 & -0.72780 & C & 2.46740 & 4.65200 & -1.99830 \\
\hline C & 4.23340 & 0.61010 & 0.11330 & C & 1.32970 & 5.50960 & -1.96750 \\
\hline C & 4.00590 & -0.59800 & 0.73880 & C & 1.44760 & 6.86910 & -2.09760 \\
\hline C & 2.77960 & -1.27960 & 0.55710 & $\mathrm{H}$ & 0.33840 & 5.11650 & -1.78880 \\
\hline C & 2.56550 & -2.55340 & 1.13470 & C & 3.88910 & 6.65980 & -2.24830 \\
\hline C & 1.37360 & -3.21760 & 0.94200 & C & 2.72340 & 7.47380 & -2.27380 \\
\hline C & 0.31420 & -2.63990 & 0.19680 & C & 2.82950 & 8.86640 & -2.44130 \\
\hline C & -3.49750 & 0.87890 & -3.78320 & C & 4.08470 & 9.44390 & -2.59600 \\
\hline$N$ & -3.18200 & 2.08700 & -4.42520 & C & 5.23200 & 8.65360 & -2.58510 \\
\hline C & -2.01230 & 2.83660 & -4.22490 & C & 5.17110 & 7.26260 & -2.41270 \\
\hline C & 5.06380 & -1.17300 & 1.61400 & $\mathrm{H}$ & 4.14800 & 10.51890 & -2.72380 \\
\hline $\mathrm{N}$ & 4.79820 & -2.44150 & 2.15990 & $\mathrm{H}$ & 6.19010 & 9.14210 & -2.71220 \\
\hline C & 3.62660 & -3.18420 & 1.96160 & C & 4.91560 & 4.42720 & -1.96610 \\
\hline $\mathrm{O}$ & -4.54770 & 0.29260 & -4.00790 & C & 4.78750 & 3.05080 & -1.64210 \\
\hline $\mathrm{O}$ & -1.82890 & 3.86970 & -4.85260 & C & 5.95760 & 2.23890 & -1.71410 \\
\hline $\mathrm{O}$ & 3.50540 & -4.29150 & 2.46850 & C & 7.19400 & 2.77200 & -1.97960 \\
\hline $\mathrm{O}$ & 6.11050 & -0.58620 & 1.84630 & $\mathrm{H}$ & 5.89900 & 1.16410 & -1.61890 \\
\hline C & -4.13210 & 2.63110 & -5.40060 & C & 6.20530 & 5.00850 & -2.18500 \\
\hline C & 5.82060 & -3.06700 & 3.00560 & C & 7.35420 & 4.17130 & -2.18070 \\
\hline C & -2.06270 & -2.63430 & -0.55280 & C & 8.63320 & 4.71230 & -2.40820 \\
\hline C & -0.94930 & -3.32620 & -0.00790 & C & 8.77200 & 6.07940 & -2.62260 \\
\hline C & -1.06080 & -4.72000 & 0.26630 & C & 7.65340 & 6.90940 & -2.61920 \\
\hline C & -2.20190 & -5.39850 & -0.04500 & C & 6.35960 & 6.40940 & -2.41050 \\
\hline C & -3.37210 & -4.70330 & -0.45280 & $\mathrm{H}$ & 9.76520 & 6.47900 & -2.79480 \\
\hline C & -3.36400 & -3.28410 & -0.53080 & $\mathrm{H}$ & 7.80310 & 7.96840 & -2.79130 \\
\hline C & -4.54800 & -5.42420 & -0.79010 & C & 0.22030 & 7.70500 & -2.04640 \\
\hline C & -5.68380 & -4.77080 & -1.16450 & $\mathrm{~N}$ & 0.39030 & 9.08200 & -2.23850 \\
\hline C & -5.78530 & -3.35500 & -1.03130 & C & 1.62070 & 9.72840 & -2.44160 \\
\hline C & -4.65550 & -2.61940 & -0.58300 & C & 8.36680 & 1.86320 & -2.06900 \\
\hline C & -7.05110 & -2.68660 & -1.28010 & $\mathrm{~N}$ & 9.61440 & 2.46360 & -2.27770 \\
\hline C & -7.17320 & -1.30550 & -0.99120 & C & 9.83450 & 3.84170 & -2.44060 \\
\hline C & -6.11220 & -0.62440 & -0.31960 & C & -6.64290 & 7.91050 & 0.88860 \\
\hline C & -4.88550 & -1.28460 & -0.04590 & $\mathrm{~N}$ & -6.86320 & 9.24250 & 1.26480 \\
\hline C & -8.18310 & -3.36880 & -1.79590 & C & -8.09460 & 9.91540 & 1.19820 \\
\hline C & -9.38180 & -2.72330 & -2.00490 & C & -13.52750 & 3.27790 & -4.40480 \\
\hline C & -9.51070 & -1.34050 & -1.73490 & $\mathrm{~N}$ & -14.68790 & 3.97520 & -4.76510 \\
\hline C & -8.39710 & -0.61930 & -1.24590 & C & -15.09640 & 5.20660 & -4.22430 \\
\hline
\end{tabular}




\begin{tabular}{|c|c|c|c|}
\hline C & -10.75890 & -0.68910 & -1.86380 \\
\hline C & -10.86520 & 0.66580 & -1.62670 \\
\hline C & -9.73690 & 1.45480 & -1.27300 \\
\hline C & -8.53170 & 0.77170 & -0.96390 \\
\hline C & -10.55330 & -3.50020 & -2.48630 \\
\hline$N$ & -11.77290 & -2.81620 & -2.56720 \\
\hline C & -11.97400 & -1.46260 & -2.23990 \\
\hline O & -13.08700 & -0.95900 & -2.27500 \\
\hline O & -10.48920 & -4.68590 & -2.78290 \\
\hline C & -12.92540 & -3.60930 & -3.00890 \\
\hline $\mathrm{H}$ & 0.26340 & 3.93210 & -3.52130 \\
\hline $\mathrm{H}$ & -3.65300 & -1.34090 & -2.41190 \\
\hline $\mathrm{H}$ & 5.17100 & 1.10270 & 0.32690 \\
\hline $\mathrm{H}$ & 1.27300 & -4.18600 & 1.41340 \\
\hline $\mathrm{H}$ & -3.65590 & 2.70210 & -6.38110 \\
\hline $\mathrm{H}$ & -4.98760 & 1.96150 & -5.43960 \\
\hline $\mathrm{H}$ & -4.43910 & 3.63390 & -5.09700 \\
\hline $\mathrm{H}$ & 6.65450 & -2.37430 & 3.08590 \\
\hline $\mathrm{H}$ & 5.40210 & -3.28270 & 3.99080 \\
\hline $\mathrm{H}$ & 6.14530 & -4.00880 & 2.55810 \\
\hline $\mathrm{H}$ & -0.20170 & -5.26780 & 0.63100 \\
\hline $\mathrm{H}$ & -2.24520 & -6.47980 & 0.04760 \\
\hline $\mathrm{H}$ & -4.51350 & -6.50980 & -0.77760 \\
\hline $\mathrm{H}$ & -6.54060 & -5.34770 & -1.48450 \\
\hline $\mathrm{H}$ & -8.15710 & -4.42450 & -2.03030 \\
\hline $\mathrm{H}$ & -11.85550 & 1.09420 & -1.68760 \\
\hline $\mathrm{H}$ & -12.71660 & -4.04360 & -3.98840 \\
\hline $\mathrm{H}$ & -13.10920 & -4.42440 & -2.30530 \\
\hline $\mathrm{H}$ & -13.78600 & -2.94700 & -3.05620 \\
\hline C & -6.34140 & 0.70240 & 0.15510 \\
\hline C & -7.49910 & 1.43800 & -0.23740 \\
\hline C & -7.68320 & 2.78100 & 0.19360 \\
\hline C & -6.83410 & 3.24820 & 1.23360 \\
\hline C & -5.72990 & 2.53100 & 1.64730 \\
\hline C & -5.42100 & 1.28460 & 1.05520 \\
\hline C & -3.98790 & -0.66390 & 0.86250 \\
\hline C & -4.25900 & 0.56510 & 1.42360 \\
\hline C & -3.33480 & 1.12360 & 2.44340 \\
\hline N & -3.71750 & 2.33040 & 3.04470 \\
\hline C & -4.88070 & 3.06510 & 2.74600 \\
\hline O & -5.16540 & 4.08290 & 3.35940 \\
\hline O & -2.29390 & 0.57110 & 2.77530 \\
\hline C & -2.81960 & 2.84060 & 4.08550 \\
\hline $\mathrm{H}$ & -7.04300 & 4.16760 & 1.76160 \\
\hline $\mathrm{H}$ & -3.23040 & 3.77990 & 4.44690 \\
\hline $\mathrm{H}$ & -2.74560 & 2.11630 & 4.89990 \\
\hline $\mathrm{H}$ & -1.82160 & 2.99020 & 3.66900 \\
\hline $\mathrm{H}$ & -3.09160 & -1.17250 & 1.19170 \\
\hline C & -9.76330 & 2.90460 & -1.15440 \\
\hline C & -8.75040 & 3.56790 & -0.41120 \\
\hline C & -10.99800 & 5.03480 & -1.40630 \\
\hline C & -10.80510 & 3.68450 & -1.78960 \\
\hline C & -11.62270 & 3.17660 & -2.84350 \\
\hline C & -12.70550 & 3.87130 & -3.31760 \\
\hline $\mathrm{H}$ & -11.39220 & 2.23840 & -3.32820 \\
\hline C & -13.03410 & 5.14910 & -2.78530 \\
\hline C & -12.14230 & 5.76290 & -1.86360 \\
\hline C & -12.39360 & 7.09710 & -1.42750 \\
\hline C & -13.57790 & 7.71900 & -1.85300 \\
\hline C & -14.46560 & 7.09370 & -2.72740 \\
\hline C & -14.18910 & 5.82270 & -3.22180 \\
\hline $\mathrm{H}$ & -13.80920 & 8.72450 & -1.52290 \\
\hline $\mathrm{H}$ & -15.36730 & 7.59480 & -3.06170 \\
\hline C & -9.99310 & 5.70320 & -0.64290 \\
\hline C & -8.80240 & 5.01410 & -0.28730 \\
\hline C & -7.69500 & 5.81050 & 0.13360 \\
\hline
\end{tabular}

$\begin{array}{cccc}\text { O } & -16.13420 & 5.74650 & -4.58290 \\ \text { O } & -13.23870 & 2.22930 & -4.96420 \\ \text { O } & -5.52370 & 7.42530 & 0.96470 \\ \text { O } & -8.20840 & 11.07560 & 1.57040 \\ \text { O } & -0.89580 & 7.24140 & -1.85980 \\ \text { O } & 1.68600 & 10.93930 & -2.60140 \\ \text { O } & 8.27790 & 0.64830 & -1.96260 \\ \text { O } & 10.95900 & 4.29080 & -2.61350 \\ \mathrm{C} & -0.83380 & 9.88910 & -2.21820 \\ \mathrm{C} & 10.76640 & 1.55730 & -2.32940 \\ \mathrm{C} & -5.69210 & 9.96870 & 1.76540 \\ \mathrm{C} & -15.51870 & 3.34980 & -5.79980 \\ \mathrm{H} & -0.55160 & 10.92460 & -2.39190 \\ \mathrm{H} & -1.51760 & 9.53940 & -2.99450 \\ \mathrm{H} & -1.33130 & 9.78160 & -1.25200 \\ \mathrm{H} & 11.65470 & 2.15890 & -2.50520 \\ \mathrm{H} & 10.85080 & 1.01300 & -1.38630 \\ \mathrm{H} & 10.62770 & 0.83010 & -3.13200 \\ \mathrm{H} & -5.99770 & 10.99200 & 1.96860 \\ \mathrm{H} & -5.32020 & 9.49200 & 2.67530 \\ \mathrm{H} & -4.89810 & 9.94130 & 1.01670 \\ \mathrm{H} & -16.36950 & 4.00140 & -5.98180 \\ \mathrm{H} & -14.93300 & 3.21570 & -6.71160 \\ \mathrm{H} & -15.85280 & 2.36660 & -5.46120 \\ & & & \end{array}$



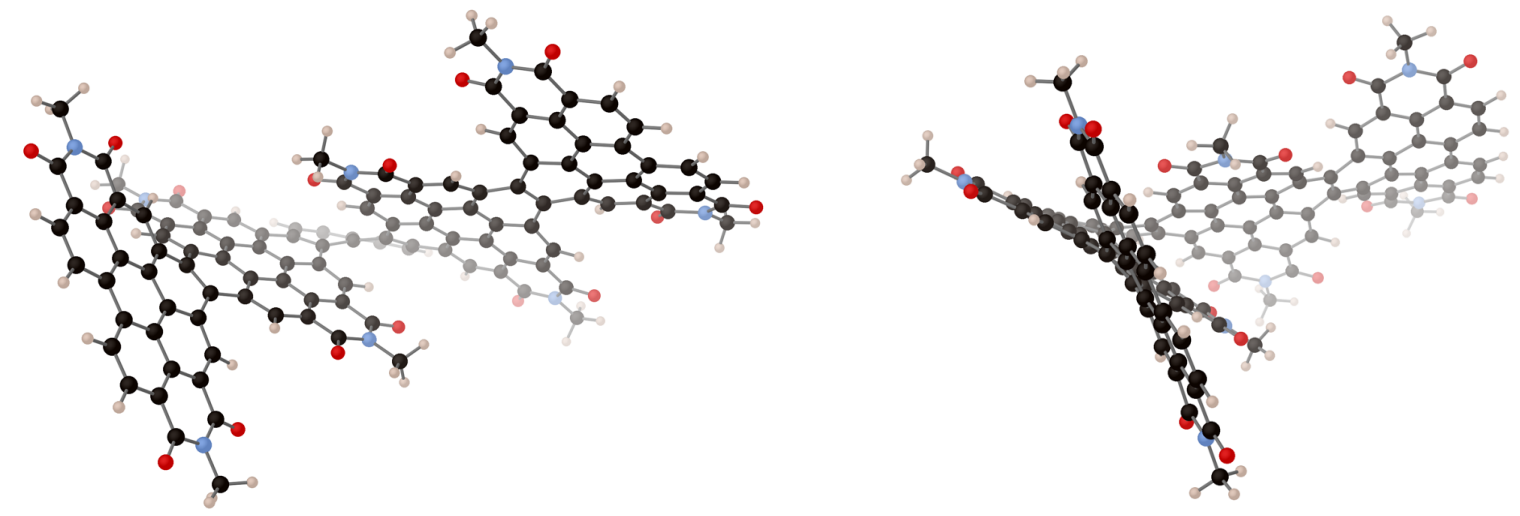

Figure S20. DFT-optimized (B3LYP/6-31G**) structure of $M$ M-M[6]-MP from different perspectives $\left(\Delta \mathrm{G}_{298.15 \mathrm{~K}}=-6166.6393\right.$ hartrees $)$.

Cartesian Coordinates of $\mathbf{M M - M}$ 6]-MP (from left to right: atom; $x, y, z$ in $\AA$ )

\begin{tabular}{|c|c|c|c|}
\hline C & 0.47880 & 1.19600 & -1.71290 \\
\hline C & -0.70990 & 0.41480 & -1.82040 \\
\hline C & -1.81160 & 0.92130 & -2.54870 \\
\hline C & -1.72910 & 2.20440 & -3.13700 \\
\hline C & -0.57350 & 2.94990 & -3.03210 \\
\hline C & 0.57850 & 2.45320 & -2.36640 \\
\hline C & -0.80960 & -0.86420 & -1.19540 \\
\hline C & -2.03140 & -1.58690 & -1.22780 \\
\hline C & -3.08870 & -1.07300 & -2.02240 \\
\hline C & -2.98710 & 0.14110 & -2.66580 \\
\hline C & 1.57820 & 0.69790 & -0.94980 \\
\hline C & 1.52590 & -0.62710 & -0.43390 \\
\hline C & 0.34140 & -1.41130 & -0.54950 \\
\hline C & 2.71030 & 1.51580 & -0.70270 \\
\hline C & 3.85400 & 0.91930 & -0.10880 \\
\hline C & 3.84030 & -0.39110 & 0.32200 \\
\hline C & 2.66800 & -1.17350 & 0.19760 \\
\hline C & 2.63210 & -2.50890 & 0.66380 \\
\hline C & 1.48820 & -3.26620 & 0.53370 \\
\hline C & 0.31330 & -2.73770 & -0.05910 \\
\hline C & -4.13640 & 0.64150 & -3.46330 \\
\hline$N$ & -4.01200 & 1.92780 & -4.00720 \\
\hline C & -2.89290 & 2.76990 & -3.87320 \\
\hline C & 5.07800 & -0.97830 & 0.90280 \\
\hline$N$ & 4.97980 & -2.30260 & 1.36590 \\
\hline C & 3.83450 & -3.10700 & 1.29970 \\
\hline $\mathrm{O}$ & -5.16100 & -0.00500 & -3.63710 \\
\hline $\mathrm{O}$ & -2.88310 & 3.89580 & -4.34920 \\
\hline $\mathrm{O}$ & 3.85540 & -4.24770 & 1.74200 \\
\hline $\mathrm{O}$ & 6.12500 & -0.35240 & 0.98120 \\
\hline C & -5.16400 & 2.41460 & -4.77380 \\
\hline C & 6.16210 & -2.91800 & 1.97850 \\
\hline C & -2.13060 & -2.85510 & -0.51980 \\
\hline C & -0.92370 & -3.49220 & -0.12300 \\
\hline C & -0.93280 & -4.88340 & 0.18350 \\
\hline C & -2.07700 & -5.61050 & 0.04230 \\
\hline C & -3.31950 & -4.97100 & -0.21470 \\
\hline C & -3.38740 & -3.55080 & -0.31040 \\
\hline C & -4.48950 & -5.75790 & -0.38550 \\
\hline C & -5.68810 & -5.16160 & -0.63970 \\
\hline C & -5.83010 & -3.74930 & -0.52130 \\
\hline C & -4.70750 & -2.94320 & -0.19200 \\
\hline C & -7.13910 & -3.14980 & -0.68440 \\
\hline C & -7.36330 & -1.84060 & -0.20410 \\
\hline C & -6.28950 & -1.09510 & 0.36830 \\
\hline
\end{tabular}

\begin{tabular}{|c|c|c|c|}
\hline $\mathrm{H}$ & -15.50740 & 6.56690 & 2.61550 \\
\hline $\mathrm{H}$ & -17.29390 & 5.09670 & 1.77020 \\
\hline & -11.22730 & 4.08490 & 2.49100 \\
\hline C & -9.90970 & 3.55320 & 2.55570 \\
\hline C & -9.02000 & 4.15270 & 3.49780 \\
\hline C & -9.32330 & 5.32450 & 4.14530 \\
\hline & -8.08400 & 3.68470 & 3.76230 \\
\hline C & -10.56910 & 5.97520 & 3.92340 \\
\hline C & -11.55450 & 5.32320 & 3.13370 \\
\hline C & -12.85600 & 5.89980 & 3.02350 \\
\hline C & -13.07660 & 7.15720 & 3.60510 \\
\hline C & -12.09080 & 7.80480 & 4.34720 \\
\hline C & -10.84860 & 7.20890 & 4.53930 \\
\hline $\mathrm{H}$ & -14.04500 & 7.63230 & 3.51470 \\
\hline $\mathrm{H}$ & -12.28300 & 8.76400 & 4.81510 \\
\hline C & 2.70790 & 2.88340 & -1.18930 \\
\hline C & 1.79620 & 3.23480 & -2.21140 \\
\hline C & 3.10410 & 5.29950 & -2.63100 \\
\hline C & 2.12260 & 4.36660 & -3.05300 \\
\hline C & 1.59190 & 4.50190 & -4.36820 \\
\hline C & 1.92530 & 5.56160 & -5.17140 \\
\hline $\mathrm{H}$ & 0.95790 & 3.73820 & -4.79730 \\
\hline C & 3.40330 & 6.45040 & -3.42590 \\
\hline C & 2.79760 & 6.58370 & -4.70490 \\
\hline C & 3.08200 & 7.69610 & -5.51760 \\
\hline C & 3.93870 & 8.68530 & -5.04770 \\
\hline C & 4.52620 & 8.57080 & -3.78980 \\
\hline C & 4.29570 & 7.46200 & -2.96140 \\
\hline $\mathrm{H}$ & 4.13950 & 9.54100 & -5.68290 \\
\hline $\mathrm{H}$ & 5.18430 & 9.36460 & -3.46040 \\
\hline C & 3.77300 & 5.11170 & -1.38420 \\
\hline C & 3.58820 & 3.90540 & -0.66330 \\
\hline C & 4.16060 & 3.80620 & 0.63900 \\
\hline C & 4.94590 & 4.80410 & 1.15870 \\
\hline $\mathrm{H}$ & 3.94280 & 2.96470 & 1.28160 \\
\hline C & 4.63890 & 6.13460 & -0.88370 \\
\hline C & 5.23470 & 5.97180 & 0.39730 \\
\hline C & 6.07320 & 6.97120 & 0.92500 \\
\hline C & 6.34090 & 8.11090 & 0.17380 \\
\hline C & 5.77290 & 8.27290 & -1.08820 \\
\hline C & 4.91210 & 7.31230 & -1.64210 \\
\hline $\mathrm{H}$ & 6.99370 & 8.86740 & 0.59500 \\
\hline $\mathrm{H}$ & 6.00810 & 9.17270 & -1.64190 \\
\hline C & 1.36920 & 5.62070 & -6.54630 \\
\hline$N$ & 1.67940 & 6.75770 & -7.30280 \\
\hline
\end{tabular}




\begin{tabular}{|c|c|c|c|}
\hline C & -4.96250 & -1.61440 & 0.36160 \\
\hline C & -8.18820 & -3.81840 & -1.36510 \\
\hline C & -9.40670 & -3.21050 & -1.57290 \\
\hline C & -9.66620 & -1.93160 & -1.02790 \\
\hline C & -8.66050 & -1.25990 & -0.29570 \\
\hline C & -10.89690 & -1.27670 & -1.26260 \\
\hline C & -11.16960 & -0.06930 & -0.65480 \\
\hline C & -10.26540 & 0.52770 & 0.26600 \\
\hline C & -8.95550 & -0.01570 & 0.33290 \\
\hline C & -10.44200 & -3.89170 & -2.39390 \\
\hline$N$ & -11.61720 & -3.17190 & -2.65040 \\
\hline C & -11.90880 & -1.88190 & -2.17060 \\
\hline O & -12.94190 & -1.30550 & -2.47670 \\
\hline O & -10.29540 & -5.01790 & -2.84890 \\
\hline C & -12.61140 & -3.84440 & -3.49450 \\
\hline $\mathrm{H}$ & -0.59620 & 3.94450 & -3.45280 \\
\hline $\mathrm{H}$ & -4.00410 & -1.63110 & -2.15400 \\
\hline $\mathrm{H}$ & 4.78500 & 1.46280 & -0.01510 \\
\hline $\mathrm{H}$ & 1.52270 & -4.27250 & 0.92780 \\
\hline $\mathrm{H}$ & -4.93820 & 3.42470 & -5.10640 \\
\hline $\mathrm{H}$ & -5.34190 & 1.75980 & -5.62970 \\
\hline $\mathrm{H}$ & -6.05700 & 2.40650 & -4.14560 \\
\hline $\mathrm{H}$ & 6.97310 & -2.19570 & 1.93210 \\
\hline $\mathrm{H}$ & 5.94520 & -3.18450 & 3.01520 \\
\hline $\mathrm{H}$ & 6.42510 & -3.82950 & 1.43810 \\
\hline $\mathrm{H}$ & -0.01140 & -5.38590 & 0.44660 \\
\hline $\mathrm{H}$ & -2.06280 & -6.69070 & 0.15520 \\
\hline $\mathrm{H}$ & -4.40130 & -6.83870 & -0.34700 \\
\hline $\mathrm{H}$ & -6.56140 & -5.77270 & -0.82980 \\
\hline $\mathrm{H}$ & -8.04350 & -4.79770 & -1.80220 \\
\hline $\mathrm{H}$ & -12.10110 & 0.40790 & -0.92050 \\
\hline $\mathrm{H}$ & -12.18540 & -4.05070 & -4.47890 \\
\hline $\mathrm{H}$ & -12.89660 & -4.79470 & -3.03910 \\
\hline $\mathrm{H}$ & -13.47200 & -3.18600 & -3.58250 \\
\hline C & -6.57070 & 0.19760 & 0.91100 \\
\hline C & -7.90200 & 0.71860 & 0.95610 \\
\hline C & -8.16780 & 2.00040 & 1.51750 \\
\hline C & -7.04300 & 2.81970 & 1.80670 \\
\hline C & -5.75540 & 2.32480 & 1.77870 \\
\hline C & -5.50330 & 0.99020 & 1.38870 \\
\hline C & -3.95160 & -0.82910 & 0.97450 \\
\hline C & -4.19680 & 0.44770 & 1.43260 \\
\hline C & -3.06070 & 1.26750 & 1.93250 \\
\hline$N$ & -3.35610 & 2.60500 & 2.24400 \\
\hline C & -4.62300 & 3.20680 & 2.16250 \\
\hline $\mathrm{O}$ & -4.75690 & 4.39450 & 2.41680 \\
\hline O & -1.92440 & 0.82380 & 2.03430 \\
\hline C & -2.26510 & 3.47570 & 2.69430 \\
\hline $\mathrm{H}$ & -7.15230 & 3.86510 & 2.05040 \\
\hline $\mathrm{H}$ & -2.48830 & 3.86110 & 3.69150 \\
\hline $\mathrm{H}$ & -1.35310 & 2.88440 & 2.70850 \\
\hline $\mathrm{H}$ & -2.16510 & 4.32420 & 2.01430 \\
\hline $\mathrm{H}$ & -2.94690 & -1.20570 & 1.08430 \\
\hline C & -10.59620 & 1.66860 & 1.10680 \\
\hline C & -9.55470 & 2.41300 & 1.72370 \\
\hline C & -12.26890 & 3.32570 & 1.88000 \\
\hline C & -11.97730 & 2.04900 & 1.33940 \\
\hline C & -13.08000 & 1.17530 & 1.10240 \\
\hline C & -14.37670 & 1.61860 & 1.13860 \\
\hline $\mathrm{H}$ & -12.93070 & 0.12430 & 0.90450 \\
\hline C & -14.67060 & 2.96960 & 1.46940 \\
\hline C & -13.61520 & 3.81400 & 1.90820 \\
\hline C & -13.92060 & 5.12150 & 2.38570 \\
\hline C & -15.24800 & 5.56720 & 2.29050 \\
\hline C & -16.26900 & 4.74500 & 1.81750 \\
\hline C & -15.99690 & 3.43580 & 1.43430 \\
\hline
\end{tabular}

$\begin{array}{cccc}\mathrm{C} & 2.49270 & 7.82000 & -6.87440 \\ \mathrm{C} & 5.47060 & 4.66320 & 2.54200 \\ \mathrm{~N} & 6.31470 & 5.68110 & 3.00200 \\ \mathrm{C} & 6.66520 & 6.83360 & 2.27920 \\ \mathrm{C} & -8.34180 & 5.90000 & 5.10280 \\ \mathrm{~N} & -8.66750 & 7.14130 & 5.66520 \\ \mathrm{C} & -9.85120 & 7.85940 & 5.42600 \\ \mathrm{C} & -15.47410 & 0.66560 & 0.83240 \\ \mathrm{~N} & -16.76840 & 1.19930 & 0.77010 \\ \mathrm{C} & -17.10910 & 2.53790 & 1.02960 \\ \mathrm{O} & -18.26490 & 2.92970 & 0.94580 \\ \mathrm{O} & -15.28620 & -0.52450 & 0.62410 \\ \mathrm{O} & -7.29300 & 5.34520 & 5.39820 \\ \mathrm{O} & -10.05240 & 8.94900 & 5.94480 \\ \mathrm{O} & 0.66790 & 4.73770 & -7.01870 \\ \mathrm{O} & 2.71190 & 8.78480 & -7.59330 \\ \mathrm{O} & 5.20030 & 3.71290 & 3.26220 \\ \mathrm{O} & 7.40850 & 7.68150 & 2.75280 \\ \mathrm{C} & 1.09470 & 6.81790 & -8.64670 \\ \mathrm{C} & 6.84840 & 5.51160 & 4.35760 \\ \mathrm{C} & -7.67240 & 7.71280 & 6.57860 \\ \mathrm{C} & -17.83530 & 0.26070 & 0.40480 \\ \mathrm{H} & 1.38120 & 7.76820 & -9.09030 \\ \mathrm{H} & 1.46520 & 5.98660 & -9.25070 \\ \mathrm{H} & 0.00840 & 6.73420 & -8.57860 \\ \mathrm{H} & 7.50300 & 6.35470 & 4.56340 \\ \mathrm{H} & 6.02790 & 5.47850 & 5.07780 \\ \mathrm{H} & 7.39780 & 4.57030 & 4.42110 \\ \mathrm{H} & -8.05590 & 8.66720 & 6.93050 \\ \mathrm{H} & -7.50660 & 7.03160 & 7.41590 \\ \mathrm{H} & -6.72420 & 7.84820 & 6.05430 \\ \mathrm{H} & -18.77120 & 0.81370 & 0.38500 \\ \mathrm{H} & -17.62420 & -0.17420 & -0.57440 \\ \mathrm{H} & -17.88240 & -0.54910 & 1.13590\end{array}$



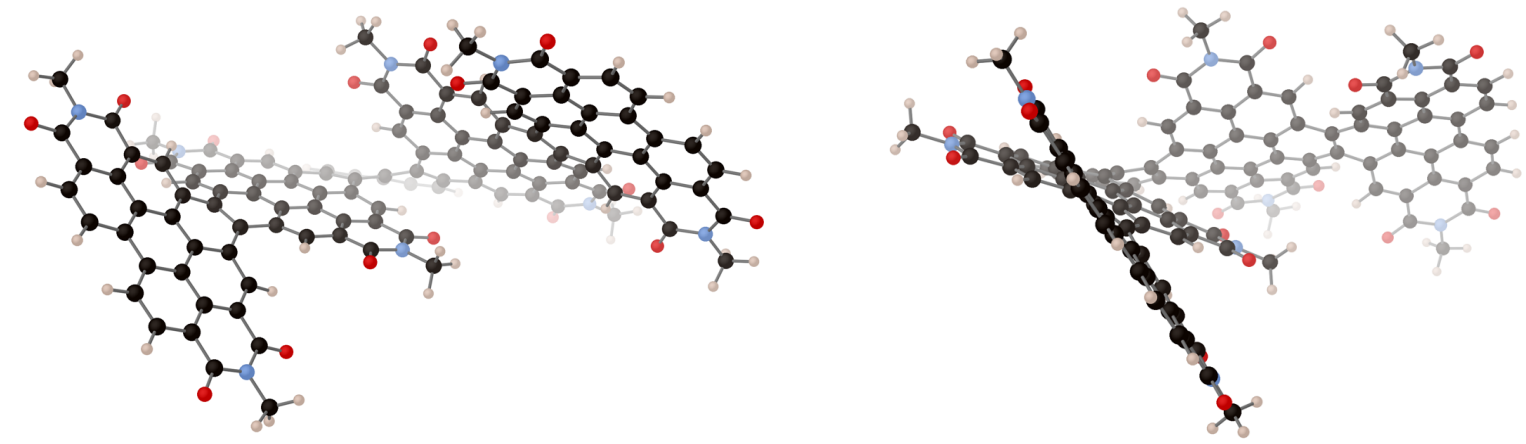

Figure S21. DFT-optimized (B3LYP/6-31G**) structure of $\mathbf{M M - M}$ 6]-PM from different perspectives $\left(\Delta \mathrm{G}_{298.15 \mathrm{~K}}=-6166.6381\right.$ hartrees$)$.

Cartesian Coordinates of $\mathbf{M M - M}$ 6]-PM (from left to right: atom; $x, y, z$ in $\AA$ )

\begin{tabular}{|c|c|c|c|}
\hline C & 0.80810 & 1.12970 & -1.56230 \\
\hline C & -0.45070 & 0.45590 & -1.52830 \\
\hline C & -1.59030 & 1.09840 & -2.06340 \\
\hline C & -1.48060 & 2.41420 & -2.56940 \\
\hline C & -0.25910 & 3.05210 & -2.60900 \\
\hline C & 0.92930 & 2.40600 & -2.17900 \\
\hline C & -0.57320 & -0.86440 & -0.99240 \\
\hline C & -1.83670 & -1.52110 & -0.96420 \\
\hline C & -2.92910 & -0.86670 & -1.59130 \\
\hline C & -2.82590 & 0.40920 & -2.09780 \\
\hline C & 1.95970 & 0.49570 & -1.00330 \\
\hline C & 1.85690 & -0.84270 & -0.52860 \\
\hline C & 0.60550 & -1.52830 & -0.52990 \\
\hline C & 3.19560 & 1.18950 & -0.93090 \\
\hline C & 4.34980 & 0.46350 & -0.53220 \\
\hline C & 4.26960 & -0.84880 & -0.11720 \\
\hline C & 3.02190 & -1.51430 & -0.08520 \\
\hline C & 2.93160 & -2.86080 & 0.33930 \\
\hline C & 1.72320 & -3.52050 & 0.31830 \\
\hline C & 0.53920 & -2.88220 & -0.13210 \\
\hline C & -4.03600 & 1.05360 & -2.67690 \\
\hline$N$ & -3.87880 & 2.37600 & -3.12250 \\
\hline C & -2.68480 & 3.11420 & -3.09020 \\
\hline C & 5.51840 & -1.56610 & 0.26440 \\
\hline $\mathrm{N}$ & 5.36260 & -2.89110 & 0.70990 \\
\hline C & 4.14880 & -3.58580 & 0.79150 \\
\hline $\mathrm{O}$ & -5.11690 & 0.48190 & -2.74090 \\
\hline $\mathrm{O}$ & -2.66520 & 4.26920 & -3.49000 \\
\hline $\mathrm{O}$ & 4.12370 & -4.73900 & 1.19990 \\
\hline $\mathrm{O}$ & 6.62030 & -1.04120 & 0.20130 \\
\hline C & -5.04300 & 3.06830 & -3.68530 \\
\hline C & 6.55720 & -3.63210 & 1.13020 \\
\hline C & -1.93940 & -2.87710 & -0.43320 \\
\hline C & -0.72820 & -3.58110 & -0.19250 \\
\hline C & -0.73860 & -5.00130 & -0.09290 \\
\hline C & -1.88980 & -5.69990 & -0.28140 \\
\hline C & -3.13160 & -5.02500 & -0.39710 \\
\hline C & -3.19510 & -3.60490 & -0.29190 \\
\hline C & -4.30920 & -5.78250 & -0.63000 \\
\hline C & -5.51600 & -5.16810 & -0.76410 \\
\hline C & -5.65460 & -3.77960 & -0.48280 \\
\hline C & -4.51430 & -3.02980 & -0.08260 \\
\hline C & -6.96440 & -3.15860 & -0.53970 \\
\hline C & -7.12050 & -1.83270 & -0.07250 \\
\hline C & -6.02770 & -1.17050 & 0.56590 \\
\hline C & -4.74870 & -1.78530 & 0.63740 \\
\hline C & -8.09890 & -3.83180 & -1.06040 \\
\hline
\end{tabular}

\begin{tabular}{|c|c|c|c|}
\hline C & -10.25710 & 4.89500 & 1.41350 \\
\hline C & -9.00150 & 4.24710 & 1.58070 \\
\hline C & -7.91160 & 5.05960 & 2.01560 \\
\hline C & -8.09620 & 6.33560 & 2.48490 \\
\hline $\mathrm{H}$ & -6.89450 & 4.70020 & 1.97950 \\
\hline C & -9.39090 & 6.92450 & 2.50210 \\
\hline C & -10.47360 & 6.22230 & 1.90760 \\
\hline C & -11.74290 & 6.86480 & 1.78830 \\
\hline C & -11.90520 & 8.12810 & 2.37690 \\
\hline C & -10.84540 & 8.79140 & 2.99240 \\
\hline C & -9.58070 & 8.21340 & 3.03300 \\
\hline $\mathrm{H}$ & -12.86410 & 8.62860 & 2.32750 \\
\hline $\mathrm{H}$ & -10.97640 & 9.77940 & 3.41990 \\
\hline C & 3.26460 & 2.56060 & -1.40320 \\
\hline C & 2.23540 & 3.04610 & -2.24230 \\
\hline C & 3.70500 & 4.93470 & -2.90470 \\
\hline C & 2.54100 & 4.15320 & -3.12960 \\
\hline C & 1.79080 & 4.40470 & -4.31650 \\
\hline C & 2.10850 & 5.42910 & -5.17240 \\
\hline $\mathrm{H}$ & 0.97550 & 3.75890 & -4.60930 \\
\hline C & 4.01620 & 6.04530 & -3.75270 \\
\hline C & 3.20440 & 6.29440 & -4.89310 \\
\hline C & 3.50190 & 7.36370 & -5.75760 \\
\hline C & 4.58270 & 8.19330 & -5.47830 \\
\hline C & 5.37820 & 7.96510 & -4.35780 \\
\hline C & 5.12860 & 6.89850 & -3.48080 \\
\hline $\mathrm{H}$ & 4.79380 & 9.01280 & -6.15640 \\
\hline $\mathrm{H}$ & 6.21150 & 8.63200 & -4.17630 \\
\hline C & 4.56640 & 4.62810 & -1.80630 \\
\hline C & 4.34770 & 3.45160 & -1.04810 \\
\hline C & 5.12370 & 3.25570 & 0.13240 \\
\hline C & 6.13780 & 4.11220 & 0.47700 \\
\hline $\mathrm{H}$ & 4.90280 & 2.44960 & 0.81890 \\
\hline C & 5.65510 & 5.50120 & -1.48970 \\
\hline C & 6.45250 & 5.23090 & -0.34350 \\
\hline C & 7.52190 & 6.07900 & -0.00190 \\
\hline C & 7.80930 & 7.17680 & -0.80520 \\
\hline C & 7.03720 & 7.44710 & -1.93230 \\
\hline C & 5.95390 & 6.63550 & -2.30080 \\
\hline $\mathrm{H}$ & 8.63870 & 7.81800 & -0.52790 \\
\hline $\mathrm{H}$ & 7.29260 & 8.31390 & -2.52840 \\
\hline C & 1.30560 & 5.61200 & -6.41230 \\
\hline $\mathrm{N}$ & 1.64990 & 6.69710 & -7.22830 \\
\hline C & 2.69120 & 7.60670 & -6.97770 \\
\hline C & 6.89600 & 3.86510 & 1.73280 \\
\hline $\mathrm{N}$ & 7.96030 & 4.73340 & 2.00660 \\
\hline C & 8.34020 & 5.82890 & 1.21270 \\
\hline
\end{tabular}




\begin{tabular}{|c|c|c|c|}
\hline C & -9.34150 & -3.23780 & -1.08240 \\
\hline C & -9.51250 & -1.91580 & -0.60910 \\
\hline C & -8.39460 & -1.19590 & -0.12700 \\
\hline C & -10.79600 & -1.32620 & -0.55240 \\
\hline C & -10.94630 & -0.02700 & -0.11530 \\
\hline C & -9.82870 & 0.78160 & 0.22610 \\
\hline C & -8.56650 & 0.14200 & 0.33310 \\
\hline C & -10.51090 & -4.00610 & -1.58600 \\
\hline$N$ & -11.76440 & -3.39100 & -1.47100 \\
\hline C & -12.00160 & -2.10590 & -0.94850 \\
\hline O & -13.13450 & -1.66060 & -0.84280 \\
\hline O & -10.41520 & -5.13020 & -2.06000 \\
\hline C & -12.91010 & -4.17720 & -1.94270 \\
\hline $\mathrm{H}$ & -0.24700 & 4.07120 & -2.96840 \\
\hline $\mathrm{H}$ & -3.88110 & -1.36100 & -1.70150 \\
\hline $\mathrm{H}$ & 5.33590 & 0.90530 & -0.57970 \\
\hline $\mathrm{H}$ & 1.71960 & -4.54420 & 0.66820 \\
\hline $\mathrm{H}$ & -4.83440 & 3.36280 & -4.71610 \\
\hline $\mathrm{H}$ & -5.88640 & 2.38370 & -3.64630 \\
\hline $\mathrm{H}$ & -5.25080 & 3.97150 & -3.10800 \\
\hline $\mathrm{H}$ & 7.41600 & -2.97960 & 0.99580 \\
\hline $\mathrm{H}$ & 6.45940 & -3.92830 & 2.17670 \\
\hline $\mathrm{H}$ & 6.66180 & -4.53640 & 0.52710 \\
\hline $\mathrm{H}$ & 0.19230 & -5.53820 & 0.03190 \\
\hline $\mathrm{H}$ & -1.87900 & -6.78490 & -0.32830 \\
\hline $\mathrm{H}$ & -4.22260 & -6.86060 & -0.72860 \\
\hline $\mathrm{H}$ & -6.38760 & -5.75910 & -1.01300 \\
\hline $\mathrm{H}$ & -8.03420 & -4.83730 & -1.45570 \\
\hline $\mathrm{H}$ & -11.95640 & 0.34730 & -0.03900 \\
\hline $\mathrm{H}$ & -12.79980 & -4.38820 & -3.00870 \\
\hline $\mathrm{H}$ & -12.95060 & -5.12820 & -1.40810 \\
\hline $\mathrm{H}$ & -13.80940 & -3.59450 & -1.76070 \\
\hline C & -6.26570 & 0.08100 & 1.20840 \\
\hline C & -7.49880 & 0.78050 & 1.03320 \\
\hline C & -7.70800 & 2.04730 & 1.64670 \\
\hline C & -6.77440 & 2.43600 & 2.64460 \\
\hline C & -5.58840 & 1.75790 & 2.83790 \\
\hline C & -5.28090 & 0.61000 & 2.07200 \\
\hline C & -3.78810 & -1.22290 & 1.51710 \\
\hline C & -4.05550 & -0.08000 & 2.24160 \\
\hline C & -3.06570 & 0.40470 & 3.23880 \\
\hline$N$ & -3.43800 & 1.52000 & 4.00310 \\
\hline C & -4.65030 & 2.22740 & 3.89230 \\
\hline O & -4.90500 & 3.17310 & 4.62300 \\
\hline O & -1.97880 & -0.12940 & 3.41470 \\
\hline C & -2.45970 & 1.96630 & 5.00060 \\
\hline $\mathrm{H}$ & -6.96620 & 3.27120 & 3.30080 \\
\hline $\mathrm{H}$ & -2.87170 & 2.83880 & 5.50110 \\
\hline $\mathrm{H}$ & -2.27090 & 1.16540 & 5.71880 \\
\hline $\mathrm{H}$ & -1.51710 & 2.21270 & 4.50800 \\
\hline $\mathrm{H}$ & -2.84040 & -1.71610 & 1.68630 \\
\hline C & -9.91940 & 2.20090 & 0.53260 \\
\hline C & -8.87650 & 2.83310 & 1.26080 \\
\hline C & -11.28560 & 4.26270 & 0.65100 \\
\hline C & -11.05370 & 2.98340 & 0.08700 \\
\hline C & -11.92980 & 2.55720 & -0.95620 \\
\hline C & -13.09480 & 3.22220 & -1.23920 \\
\hline $\mathrm{H}$ & -11.69470 & 1.69980 & -1.56950 \\
\hline C & -13.44400 & 4.40330 & -0.52730 \\
\hline C & -12.50790 & 4.96090 & 0.38670 \\
\hline C & -12.79120 & 6.21660 & 0.99750 \\
\hline C & -14.04260 & 6.80370 & 0.75510 \\
\hline C & -14.96880 & 6.23010 & -0.11370 \\
\hline C & -14.66760 & 5.04810 & -0.78260 \\
\hline $\mathrm{H}$ & -14.30190 & 7.74100 & 1.23160 \\
\hline $\mathrm{H}$ & -15.92400 & 6.70560 & -0.30740 \\
\hline
\end{tabular}

$\begin{array}{cccc}\mathrm{C} & -6.91780 & 7.10780 & 2.96480 \\ \mathrm{~N} & -7.17670 & 8.37260 & 3.50980 \\ \mathrm{C} & -8.44050 & 8.98060 & 3.59720 \\ \mathrm{C} & -13.98450 & 2.70040 & -2.31220 \\ \mathrm{~N} & -15.20680 & 3.36230 & -2.48860 \\ \mathrm{C} & -15.62470 & 4.50240 & -1.78090 \\ \mathrm{O} & -16.71090 & 5.02280 & -1.99500 \\ \mathrm{O} & -13.69690 & 1.73740 & -3.00860 \\ \mathrm{O} & -5.77280 & 6.68240 & 2.91080 \\ \mathrm{O} & -8.58150 & 10.09040 & 4.09260 \\ \mathrm{O} & 0.38530 & 4.87080 & -6.72770 \\ \mathrm{O} & 2.92070 & 8.53760 & -7.73690 \\ \mathrm{O} & 6.63080 & 2.95190 & 2.50120 \\ \mathrm{O} & 9.28690 & 6.54020 & 1.51990 \\ \mathrm{C} & 0.83900 & 6.87530 & -8.43740 \\ \mathrm{C} & 8.71710 & 4.45560 & 3.23220 \\ \mathrm{C} & -6.00920 & 9.10380 & 4.01320 \\ \mathrm{C} & -16.09490 & 2.80660 & -3.51600 \\ \mathrm{H} & 1.21500 & 7.75050 & -8.96140 \\ \mathrm{H} & 0.91550 & 5.98660 & -9.06730 \\ \mathrm{H} & -0.20900 & 7.01060 & -8.16270 \\ \mathrm{H} & 9.50980 & 5.19570 & 3.30710 \\ \mathrm{H} & 8.05360 & 4.51320 & 4.09760 \\ \mathrm{H} & 9.13330 & 3.44710 & 3.18760 \\ \mathrm{H} & -6.35950 & 10.05360 & 4.40940 \\ \mathrm{H} & -5.51580 & 8.51870 & 4.79190 \\ \mathrm{H} & -5.29580 & 9.26400 & 3.20220 \\ \mathrm{H} & -17.00770 & 3.39680 & -3.51980 \\ \mathrm{H} & -15.60590 & 2.85020 & -4.49170 \\ \mathrm{H} & -16.31030 & 1.76070 & -3.28880 \\ & & & \end{array}$



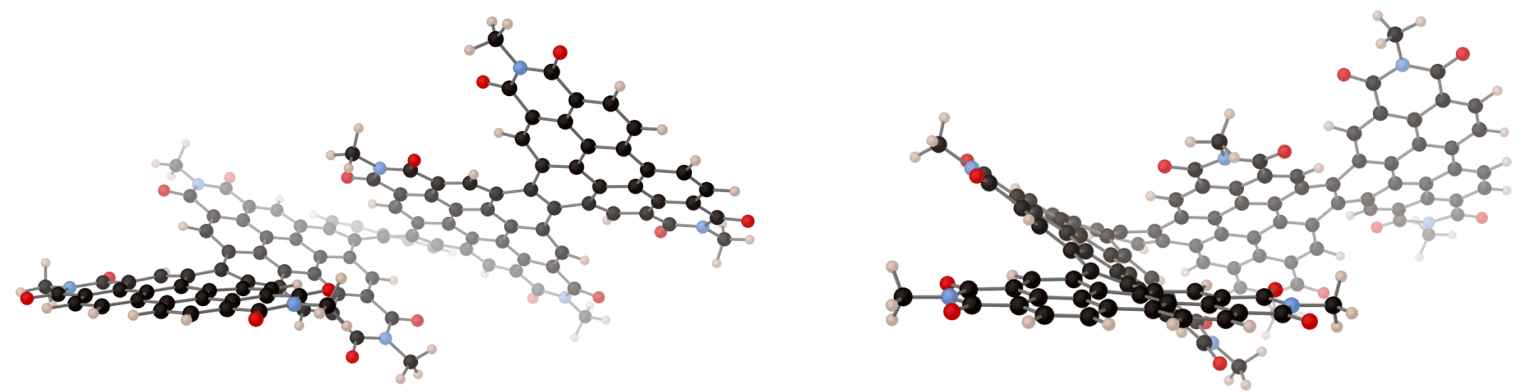

Figure S22. DFT-optimized (B3LYP/6-31G**) structure of $\boldsymbol{P P}-\mathbf{M}$ 6]-MP from different perspectives $\left(\Delta \mathrm{G}_{298.15 \mathrm{~K}}=-6166.6372\right.$ hartrees $)$.

Cartesian Coordinates of PP-M[6]-MP (from left to right: atom; x, y, z in $\AA$ )

\begin{tabular}{|c|c|c|c|}
\hline C & 0.83260 & 1.00170 & -1.69430 \\
\hline C & -0.37960 & 0.25810 & -1.81960 \\
\hline C & -1.40970 & 0.74460 & -2.65760 \\
\hline C & -1.20550 & 1.93540 & -3.39370 \\
\hline C & -0.05830 & 2.68040 & -3.21410 \\
\hline C & 0.95520 & 2.27760 & -2.30500 \\
\hline C & -0.56840 & -0.96760 & -1.11280 \\
\hline C & -1.82110 & -1.63610 & -1.15700 \\
\hline C & -2.81280 & -1.13580 & -2.03990 \\
\hline C & -2.61380 & 0.00960 & -2.77980 \\
\hline C & 1.91530 & 0.46490 & -0.93300 \\
\hline C & 1.74630 & -0.78700 & -0.27070 \\
\hline C & 0.52070 & -1.51170 & -0.36530 \\
\hline C & 3.16290 & 1.14530 & -0.87760 \\
\hline C & 4.16310 & 0.62340 & -0.01470 \\
\hline C & 4.00640 & -0.58260 & 0.63420 \\
\hline C & 2.81640 & -1.32990 & 0.47880 \\
\hline C & 2.67070 & -2.59630 & 1.09360 \\
\hline C & 1.50380 & -3.31460 & 0.94880 \\
\hline C & 0.40150 & -2.79270 & 0.22710 \\
\hline C & -3.67870 & 0.47580 & -3.70430 \\
\hline$N$ & -3.39690 & 1.62100 & -4.46250 \\
\hline C & -2.22560 & 2.39490 & -4.37450 \\
\hline C & 5.09880 & -1.07950 & 1.51490 \\
\hline$N$ & 4.90830 & -2.35120 & 2.08420 \\
\hline C & 3.77420 & -3.15640 & 1.91620 \\
\hline $\mathrm{O}$ & -4.75480 & -0.09520 & -3.82400 \\
\hline $\mathrm{O}$ & -2.06570 & 3.38700 & -5.07090 \\
\hline $\mathrm{O}$ & 3.71590 & -4.25850 & 2.44520 \\
\hline $\mathrm{O}$ & 6.11080 & -0.42950 & 1.73200 \\
\hline C & -4.43990 & 2.04450 & -5.40230 \\
\hline C & 5.97120 & -2.90660 & 2.92890 \\
\hline C & -2.00510 & -2.86050 & -0.39180 \\
\hline C & -0.85000 & -3.51580 & 0.10870 \\
\hline C & -0.92110 & -4.90060 & 0.44070 \\
\hline C & -2.06820 & -5.60390 & 0.21510 \\
\hline C & -3.27230 & -4.93780 & -0.14320 \\
\hline C & -3.29030 & -3.51940 & -0.24940 \\
\hline C & -4.45470 & -5.68160 & -0.40480 \\
\hline C & -5.61430 & -5.04510 & -0.73620 \\
\hline C & -5.72130 & -3.62970 & -0.60480 \\
\hline C & -4.58990 & -2.86800 & -0.20980 \\
\hline C & -7.00080 & -2.98200 & -0.81060 \\
\hline C & -7.19410 & -1.66980 & -0.31990 \\
\hline C & -6.12400 & -0.97670 & 0.32190 \\
\hline C & -4.81770 & -1.54060 & 0.35720 \\
\hline C & -8.05230 & -3.60690 & -1.52830 \\
\hline C & -9.23270 & -2.94270 & -1.78480 \\
\hline
\end{tabular}

\begin{tabular}{|c|c|c|c|}
\hline & -9.09810 & $3.0<540$ & 2.59950 \\
\hline C & -8.84940 & 4.06600 & 3.65850 \\
\hline & -9.14600 & 5.17460 & 4.41020 \\
\hline E & -7.96050 & 3.51520 & 3.93120 \\
\hline 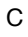 & -10.33910 & 5.91420 & 4.17920 \\
\hline & -11.29570 & 5.40430 & 3.26190 \\
\hline 0 & -12.55840 & 6.05600 & 3.13350 \\
\hline C & -12.76420 & 7.25300 & 3.83660 \\
\hline C & -11.79950 & 7.76810 & 4.70110 \\
\hline & -10.60090 & 7.09100 & 4.90390 \\
\hline $\mathrm{H}$ & -13.70380 & 7.78340 & 3.73980 \\
\hline $\mathrm{H}$ & -11.97680 & 8.68430 & 5.25350 \\
\hline C & 3.31830 & 2.40110 & -1.59370 \\
\hline C & 2.16030 & 3.05640 & -2.07480 \\
\hline C & 3.46840 & 5.14570 & -2.34640 \\
\hline C & 2.21410 & 4.48700 & -2.28900 \\
\hline C & 1.03420 & 5.28780 & -2.30190 \\
\hline C & 1.08640 & 6.64760 & -2.47180 \\
\hline $\mathrm{H}$ & 0.06170 & 4.85070 & -2.12080 \\
\hline C & 3.53880 & 6.55620 & -2.57710 \\
\hline C & 2.33430 & 7.30900 & -2.64870 \\
\hline C & 2.37460 & 8.69900 & -2.86180 \\
\hline C & 3.60200 & 9.33470 & -3.01670 \\
\hline C & 4.78820 & 8.60630 & -2.95670 \\
\hline C & 4.79220 & 7.22060 & -2.73520 \\
\hline $\mathrm{H}$ & 3.61210 & 10.40630 & -3.18270 \\
\hline $\mathrm{H}$ & 5.72300 & 9.13830 & -3.08330 \\
\hline C & 4.67300 & 4.39060 & -2.20150 \\
\hline C & 4.60690 & 3.02080 & -1.83730 \\
\hline C & 5.81870 & 2.26960 & -1.85100 \\
\hline C & 7.03260 & 2.85840 & -2.09840 \\
\hline $\mathrm{H}$ & 5.81410 & 1.19690 & -1.72270 \\
\hline C & 5.93640 & 5.03150 & -2.40770 \\
\hline C & 7.12630 & 4.25620 & -2.34410 \\
\hline C & 8.38190 & 4.85500 & -2.55400 \\
\hline C & 8.45560 & 6.21970 & -2.80960 \\
\hline C & 7.29670 & 6.99070 & -2.86460 \\
\hline C & 6.02460 & 6.43030 & -2.67590 \\
\hline $\mathrm{H}$ & 9.43170 & 6.66570 & -2.96570 \\
\hline $\mathrm{H}$ & 7.39730 & 8.05020 & -3.06620 \\
\hline C & -0.18080 & 7.42560 & -2.45380 \\
\hline$N$ & -0.07610 & 8.80040 & -2.69610 \\
\hline C & 1.12440 & 9.49780 & -2.91340 \\
\hline C & 8.25270 & 2.00960 & -2.12060 \\
\hline$N$ & 9.47310 & 2.66570 & -2.32070 \\
\hline C & 9.62710 & 4.04740 & -2.52210 \\
\hline C & -8.21780 & 5.58040 & 5.49540 \\
\hline & -8.50800 & 6.78470 & 6.15120 \\
\hline
\end{tabular}




\begin{tabular}{|c|c|c|c|}
\hline C & -9.45730 & -1.65380 & -1.24760 \\
\hline C & -8.46250 & -1.03660 & -0.45730 \\
\hline C & -10.64130 & -0.93800 & -1.53600 \\
\hline C & -10.89140 & 0.27310 & -0.92700 \\
\hline C & -10.02410 & 0.80260 & 0.06700 \\
\hline C & -8.74090 & 0.20440 & 0.18470 \\
\hline C & -10.26870 & -3.57630 & -2.64180 \\
\hline$N$ & -11.39010 & -2.79550 & -2.95590 \\
\hline C & -11.63000 & -1.48480 & -2.50110 \\
\hline $\mathrm{O}$ & -12.60750 & -0.85070 & -2.86910 \\
\hline O & -10.16350 & -4.71300 & -3.08240 \\
\hline C & -12.37810 & -3.41830 & -3.84370 \\
\hline $\mathrm{H}$ & 0.05670 & 3.56500 & -3.82510 \\
\hline $\mathrm{H}$ & -3.74780 & -1.65930 & -2.17700 \\
\hline $\mathrm{H}$ & 5.07210 & 1.17010 & 0.18840 \\
\hline $\mathrm{H}$ & 1.45220 & -4.27320 & 1.44750 \\
\hline $\mathrm{H}$ & -4.02360 & 2.82650 & -6.03220 \\
\hline $\mathrm{H}$ & -4.75610 & 1.18820 & -5.99930 \\
\hline $\mathrm{H}$ & -5.30830 & 2.42340 & -4.85740 \\
\hline $\mathrm{H}$ & 6.76880 & -2.17040 & 2.98580 \\
\hline $\mathrm{H}$ & 5.57600 & -3.12440 & 3.92320 \\
\hline $\mathrm{H}$ & 6.33880 & -3.83910 & 2.49520 \\
\hline $\mathrm{H}$ & -0.03350 & -5.42160 & 0.77550 \\
\hline $\mathrm{H}$ & -2.08830 & -6.68280 & 0.33970 \\
\hline $\mathrm{H}$ & -4.40780 & -6.76620 & -0.36940 \\
\hline $\mathrm{H}$ & -6.49070 & -5.62740 & -0.99110 \\
\hline $\mathrm{H}$ & -7.93570 & -4.59260 & -1.95930 \\
\hline $\mathrm{H}$ & -11.77790 & 0.80170 & -1.24280 \\
\hline $\mathrm{H}$ & -11.92290 & -3.63940 & -4.81190 \\
\hline $\mathrm{H}$ & -12.72320 & -4.35680 & -3.40570 \\
\hline $\mathrm{H}$ & -13.20370 & -2.72130 & -3.96280 \\
\hline C & -6.38690 & 0.30310 & 0.90060 \\
\hline C & -7.69940 & 0.86800 & 0.89730 \\
\hline C & -7.95700 & 2.11860 & 1.52330 \\
\hline C & -6.83020 & 2.88120 & 1.93410 \\
\hline C & -5.55800 & 2.34820 & 1.94290 \\
\hline C & -5.32440 & 1.03420 & 1.47760 \\
\hline C & -3.80980 & -0.80830 & 1.03650 \\
\hline C & -4.03720 & 0.45140 & 1.54660 \\
\hline C & -2.90120 & 1.20620 & 2.14010 \\
\hline$N$ & -3.17730 & 2.52370 & 2.53990 \\
\hline C & -4.42280 & 3.16670 & 2.44220 \\
\hline O & -4.53870 & 4.33630 & 2.77670 \\
\hline $\mathrm{O}$ & -1.78020 & 0.72620 & 2.24980 \\
\hline C & -2.08370 & 3.33330 & 3.08690 \\
\hline $\mathrm{H}$ & -6.92770 & 3.91240 & 2.23900 \\
\hline $\mathrm{H}$ & -2.32470 & 3.63810 & 4.10770 \\
\hline $\mathrm{H}$ & -1.18300 & 2.72470 & 3.07500 \\
\hline $\mathrm{H}$ & -1.95220 & 4.23250 & 2.48200 \\
\hline $\mathrm{H}$ & -2.82050 & -1.21730 & 1.16940 \\
\hline C & -10.36740 & 1.91080 & 0.94570 \\
\hline C & -9.33900 & 2.55750 & 1.68280 \\
\hline C & -12.01070 & 3.58190 & 1.75990 \\
\hline C & -11.74300 & 2.34480 & 1.11900 \\
\hline C & -12.87040 & 1.56020 & 0.72820 \\
\hline C & -14.14380 & 2.06920 & 0.72220 \\
\hline $\mathrm{H}$ & -12.76370 & 0.52290 & 0.44980 \\
\hline C & -14.39400 & 3.39640 & 1.16730 \\
\hline C & -13.33130 & 4.13660 & 1.75370 \\
\hline C & -13.60720 & 5.40360 & 2.34740 \\
\hline C & -14.90450 & 5.92380 & 2.21960 \\
\hline C & -15.92700 & 5.21140 & 1.59550 \\
\hline C & -15.69120 & 3.93510 & 1.09570 \\
\hline $\mathrm{H}$ & -15.14040 & 6.89600 & 2.63370 \\
\hline $\mathrm{H}$ & -16.92740 & 5.62260 & 1.51710 \\
\hline C & -10.98240 & 4.22440 & 2.51250 \\
\hline
\end{tabular}

\begin{tabular}{|c|c|c|c|}
\hline C & -9.62700 & 7.59830 & 5.90320 \\
\hline C & -15.26690 & 1.20920 & 0.26230 \\
\hline $\mathrm{N}$ & -16.52400 & 1.81990 & 0.16320 \\
\hline C & -16.81220 & 3.14830 & 0.52060 \\
\hline $\mathrm{O}$ & -17.93470 & 3.61580 & 0.38530 \\
\hline $\mathrm{O}$ & -15.12690 & 0.03180 & -0.03700 \\
\hline $\mathrm{O}$ & -7.23610 & 4.92500 & 5.81500 \\
\hline $\mathrm{O}$ & -9.79510 & 8.65190 & 6.50210 \\
\hline $\mathrm{O}$ & -1.27400 & 6.91590 & -2.24910 \\
\hline $\mathrm{O}$ & 1.13130 & 10.70290 & -3.12280 \\
\hline $\mathrm{O}$ & 8.22170 & 0.79690 & -1.96720 \\
\hline $\mathrm{O}$ & 10.73180 & 4.54920 & -2.67680 \\
\hline C & -1.33690 & 9.54930 & -2.71170 \\
\hline C & 10.67010 & 1.81810 & -2.31190 \\
\hline C & -7.55380 & 7.20040 & 7.18410 \\
\hline C & -17.60950 & 0.97910 & -0.35330 \\
\hline $\mathrm{H}$ & -1.10360 & 10.58690 & -2.93700 \\
\hline $\mathrm{H}$ & -2.00390 & 9.13010 & -3.46770 \\
\hline $\mathrm{H}$ & -1.82820 & 9.46800 & -1.73960 \\
\hline $\mathrm{H}$ & 11.52830 & 2.45360 & -2.51520 \\
\hline $\mathrm{H}$ & 10.77410 & 1.33290 & -1.33880 \\
\hline $\mathrm{H}$ & 10.57480 & 1.04080 & -3.07250 \\
\hline $\mathrm{H}$ & -7.91490 & 8.13130 & 7.61390 \\
\hline $\mathrm{H}$ & -7.47950 & 6.42450 & 7.94880 \\
\hline $\mathrm{H}$ & -6.56530 & 7.33950 & 6.74100 \\
\hline $\mathrm{H}$ & -18.51520 & 1.58010 & -0.37150 \\
\hline $\mathrm{H}$ & -17.36000 & 0.62860 & -1.35710 \\
\hline $\mathrm{H}$ & -17.73850 & 0.10670 & 0.29070 \\
\hline
\end{tabular}



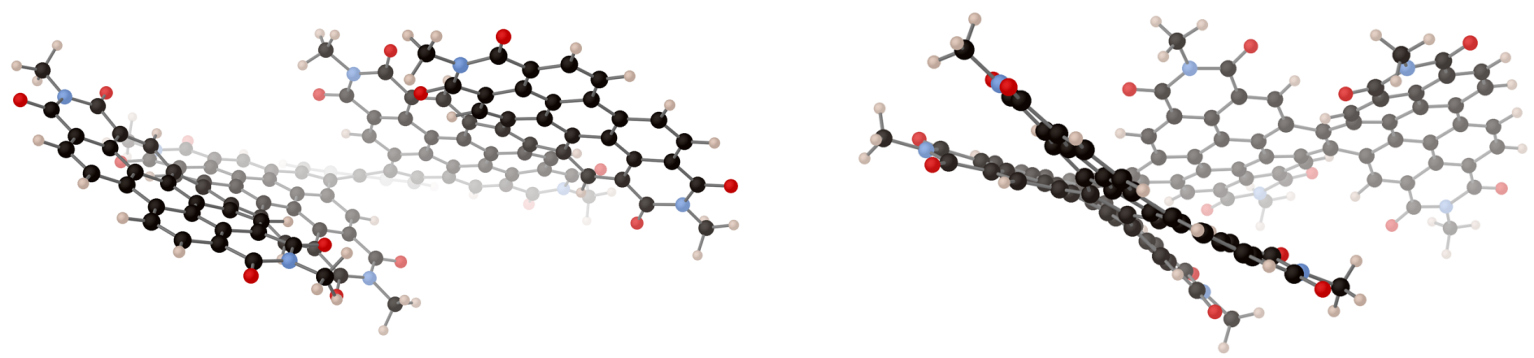

Figure S23. DFT-optimized (B3LYP/6-31G**) structure of $\boldsymbol{P M}-\boldsymbol{M}$ 6]-PM from different perspectives $\left(\Delta \mathrm{G}_{298.15 \mathrm{~K}}=-6166.6321\right.$ hartrees $)$.

Cartesian Coordinates of PM-M[6]-PM (from left to right: atom; x, y, z in $\AA$ )

\begin{tabular}{|c|c|c|c|}
\hline C & 0.52210 & 1.15710 & -1.65300 \\
\hline C & -0.61240 & 0.32660 & -1.89110 \\
\hline C & -1.66170 & 0.79890 & -2.70980 \\
\hline C & -1.50190 & 2.02980 & -3.38600 \\
\hline C & -0.41670 & 2.84070 & -3.12040 \\
\hline C & 0.56220 & 2.48790 & -2.15290 \\
\hline C & -0.69420 & -0.98300 & -1.33460 \\
\hline C & -1.89970 & -1.72050 & -1.42370 \\
\hline C & -2.93190 & -1.21370 & -2.25620 \\
\hline C & -2.80560 & -0.00960 & -2.91430 \\
\hline C & 1.66500 & 0.59870 & -1.00800 \\
\hline C & 1.66310 & -0.78430 & -0.66180 \\
\hline C & 0.47600 & -1.56520 & -0.76030 \\
\hline C & 2.83590 & 1.38250 & -0.84400 \\
\hline C & 4.04810 & 0.69480 & -0.56980 \\
\hline C & 4.06670 & -0.65130 & -0.26590 \\
\hline C & 2.86630 & -1.40010 & -0.25170 \\
\hline C & 2.86650 & -2.77400 & 0.09080 \\
\hline C & 1.70520 & -3.51560 & 0.02310 \\
\hline C & 0.48310 & -2.93790 & -0.40740 \\
\hline C & -3.86750 & 0.42260 & -3.86110 \\
\hline $\mathrm{N}$ & -3.62740 & 1.62130 & -4.55450 \\
\hline C & -2.49960 & 2.44480 & -4.40460 \\
\hline C & 5.36420 & -1.30850 & 0.04420 \\
\hline $\mathrm{N}$ & 5.29570 & -2.65110 & 0.45900 \\
\hline C & 4.13250 & -3.43150 & 0.50680 \\
\hline $\mathrm{O}$ & -4.88740 & -0.22810 & -4.04550 \\
\hline $\mathrm{O}$ & -2.37470 & 3.44930 & -5.08950 \\
\hline $\mathrm{O}$ & 4.18820 & -4.60100 & 0.86260 \\
\hline $\mathrm{O}$ & 6.43280 & -0.72090 & -0.03850 \\
\hline C & -4.61550 & 2.06740 & -5.54220 \\
\hline C & 6.53820 & -3.32420 & 0.85300 \\
\hline C & -1.97410 & -3.02960 & -0.79600 \\
\hline C & -0.75690 & -3.69140 & -0.48710 \\
\hline C & -0.77160 & -5.10550 & -0.30800 \\
\hline C & -1.92490 & -5.81800 & -0.47260 \\
\hline C & -3.17350 & -5.15670 & -0.62480 \\
\hline C & -3.22130 & -3.73740 & -0.61060 \\
\hline C & -4.37430 & -5.89780 & -0.78950 \\
\hline C & -5.57370 & -5.26030 & -0.91470 \\
\hline C & -5.68250 & -3.85560 & -0.69000 \\
\hline C & -4.51070 & -3.11010 & -0.39970 \\
\hline C & -6.98290 & -3.21120 & -0.67720 \\
\hline C & -7.09070 & -1.87050 & -0.23060 \\
\hline C & -5.94230 & -1.20120 & 0.29330 \\
\hline C & -4.66520 & -1.82200 & 0.26100 \\
\hline C & -8.16130 & -3.88530 & -1.08480 \\
\hline C & -9.39760 & -3.28210 & -1.00870 \\
\hline C & -9.52100 & -1.95200 & -0.54070 \\
\hline C & -8.36260 & -1.22640 & -0.17890 \\
\hline
\end{tabular}

\begin{tabular}{|c|c|c|c|}
\hline C & -7.66460 & 5.05730 & 1.81750 \\
\hline C & -7.79540 & 6.32720 & 2.32180 \\
\hline $\mathrm{H}$ & -6.66010 & 4.71560 & 1.61720 \\
\hline C & -9.08230 & 6.89410 & 2.53600 \\
\hline C & -10.22720 & 6.18860 & 2.07930 \\
\hline C & -11.50790 & 6.81340 & 2.15020 \\
\hline C & -11.60470 & 8.05960 & 2.78740 \\
\hline C & -10.47920 & 8.72380 & 3.27160 \\
\hline C & -9.21350 & 8.16650 & 3.12150 \\
\hline $\mathrm{H}$ & -12.56840 & 8.54220 & 2.88890 \\
\hline $\mathrm{H}$ & -10.56470 & 9.69610 & 3.74400 \\
\hline C & 2.73020 & 2.82710 & -0.99700 \\
\hline C & 1.60820 & 3.38200 & -1.66950 \\
\hline C & 2.65600 & 5.63130 & -1.53810 \\
\hline C & 1.52080 & 4.82400 & -1.82470 \\
\hline C & 0.32580 & 5.50460 & -2.21090 \\
\hline C & 0.32150 & 6.83670 & -2.53990 \\
\hline $\mathrm{H}$ & -0.62710 & 4.99640 & -2.23550 \\
\hline C & 2.66870 & 7.02500 & -1.87030 \\
\hline C & 1.51050 & 7.61220 & -2.44560 \\
\hline C & 1.51500 & 8.96120 & -2.84510 \\
\hline C & 2.67190 & 9.71800 & -2.68850 \\
\hline C & 3.79930 & 9.16650 & -2.08280 \\
\hline C & 3.81620 & 7.83910 & -1.62740 \\
\hline $\mathrm{H}$ & 2.66470 & 10.75030 & -3.02080 \\
\hline $\mathrm{H}$ & 4.66930 & 9.79730 & -1.94610 \\
\hline C & 3.76180 & 5.07400 & -0.82610 \\
\hline C & 3.73240 & 3.71090 & -0.43740 \\
\hline C & 4.66140 & 3.29180 & 0.56300 \\
\hline C & 5.68140 & 4.10140 & 0.99180 \\
\hline $\mathrm{H}$ & 4.56280 & 2.33360 & 1.05090 \\
\hline C & 4.84230 & 5.92250 & -0.41970 \\
\hline C & 5.83570 & 5.41140 & 0.45980 \\
\hline C & 6.91790 & 6.21500 & 0.86300 \\
\hline C & 7.02440 & 7.51330 & 0.37560 \\
\hline C & 6.04190 & 8.03300 & -0.46390 \\
\hline C & 4.92680 & 7.27710 & -0.85610 \\
\hline $\mathrm{H}$ & 7.86960 & 8.11690 & 0.68770 \\
\hline $\mathrm{H}$ & 6.14100 & 9.06050 & -0.79000 \\
\hline C & -0.94800 & 7.46970 & -2.98620 \\
\hline $\mathrm{N}$ & -0.86400 & 8.79540 & -3.43250 \\
\hline C & 0.29440 & 9.59030 & -3.40910 \\
\hline C & 6.61110 & 3.60310 & 2.04010 \\
\hline $\mathrm{N}$ & 7.69230 & 4.43180 & 2.36580 \\
\hline C & 7.92480 & 5.70990 & 1.83050 \\
\hline C & -6.57380 & 7.11460 & 2.63550 \\
\hline $\mathrm{N}$ & -6.76950 & 8.35070 & 3.26620 \\
\hline C & -8.01680 & 8.92960 & 3.55520 \\
\hline C & -14.23460 & 2.66870 & -1.66800 \\
\hline $\mathrm{N}$ & -15.47950 & 3.31020 & -1.66540 \\
\hline
\end{tabular}




\begin{tabular}{|c|c|c|c|}
\hline C & -10.79380 & -1.36890 & -0.34520 \\
\hline C & -10.90470 & -0.06650 & 0.09580 \\
\hline C & -9.76300 & 0.75150 & 0.31640 \\
\hline C & -8.49250 & 0.11610 & 0.28790 \\
\hline C & -10.60940 & -4.05260 & -1.38860 \\
\hline$N$ & -11.84580 & -3.43550 & -1.16080 \\
\hline C & -12.02930 & -2.16010 & -0.59600 \\
\hline $\mathrm{O}$ & -13.14500 & -1.72870 & -0.34650 \\
\hline O & -10.55880 & -5.18060 & -1.86000 \\
\hline C & -13.03170 & -4.22380 & -1.51260 \\
\hline $\mathrm{H}$ & -0.32790 & 3.74300 & -3.70720 \\
\hline $\mathrm{H}$ & -3.82480 & -1.79350 & -2.44390 \\
\hline $\mathrm{H}$ & 5.00270 & 1.19930 & -0.61460 \\
\hline $\mathrm{H}$ & 1.77350 & -4.55670 & 0.31220 \\
\hline $\mathrm{H}$ & -4.15560 & 2.11010 & -6.53200 \\
\hline $\mathrm{H}$ & -5.43800 & 1.35660 & -5.53380 \\
\hline $\mathrm{H}$ & -4.96720 & 3.06860 & -5.28530 \\
\hline $\mathrm{H}$ & 7.34340 & -2.59610 & 0.79480 \\
\hline $\mathrm{H}$ & 6.43830 & -3.71000 & 1.86940 \\
\hline $\mathrm{H}$ & 6.73550 & -4.16570 & 0.18520 \\
\hline $\mathrm{H}$ & 0.15330 & -5.63420 & -0.11520 \\
\hline $\mathrm{H}$ & -1.91030 & -6.90380 & -0.44900 \\
\hline $\mathrm{H}$ & -4.31630 & -6.98100 & -0.84490 \\
\hline $\mathrm{H}$ & -6.46400 & -5.84690 & -1.09920 \\
\hline $\mathrm{H}$ & -8.13520 & -4.89840 & -1.46260 \\
\hline $\mathrm{H}$ & -11.90270 & 0.29490 & 0.29300 \\
\hline $\mathrm{H}$ & -12.98420 & -4.50270 & -2.56700 \\
\hline $\mathrm{H}$ & -13.06210 & -5.13950 & -0.91760 \\
\hline $\mathrm{H}$ & -13.90840 & -3.61270 & -1.31390 \\
\hline C & -6.11700 & 0.06470 & 0.93160 \\
\hline C & -7.35960 & 0.76460 & 0.86490 \\
\hline C & -7.50380 & 2.04240 & 1.47490 \\
\hline C & -6.47180 & 2.45640 & 2.35830 \\
\hline C & -5.27590 & 1.77640 & 2.45130 \\
\hline C & -5.05100 & 0.61400 & 1.68010 \\
\hline C & -3.62140 & -1.23020 & 1.02020 \\
\hline C & -3.81230 & -0.06790 & 1.73360 \\
\hline C & -2.71790 & 0.44620 & 2.59630 \\
\hline$N$ & -3.00950 & 1.57120 & 3.37930 \\
\hline C & -4.23770 & 2.25750 & 3.40160 \\
\hline O & -4.42850 & 3.19660 & 4.16030 \\
\hline O & -1.61260 & -0.07670 & 2.65430 \\
\hline C & -1.93610 & 2.03420 & 4.26450 \\
\hline $\mathrm{H}$ & -6.60130 & 3.29870 & 3.02050 \\
\hline $\mathrm{H}$ & -2.29650 & 2.91430 & 4.79120 \\
\hline $\mathrm{H}$ & -1.67230 & 1.24590 & 4.97330 \\
\hline $\mathrm{H}$ & -1.04970 & 2.27330 & 3.67380 \\
\hline $\mathrm{H}$ & -2.66270 & -1.71760 & 1.11920 \\
\hline C & -9.82860 & 2.17540 & 0.62150 \\
\hline C & -8.70800 & 2.81930 & 1.21160 \\
\hline C & -11.18360 & 4.22610 & 0.92990 \\
\hline C & -11.01780 & 2.95260 & 0.32930 \\
\hline C & -12.02510 & 2.52250 & -0.58570 \\
\hline C & -13.21780 & 3.18610 & -0.71490 \\
\hline $\mathrm{H}$ & -11.86570 & 1.67630 & -1.23730 \\
\hline C & -13.47820 & 4.35950 & 0.04600 \\
\hline C & -12.43340 & 4.91840 & 0.83210 \\
\hline C & -12.64390 & 6.16410 & 1.49360 \\
\hline C & -13.91970 & 6.74480 & 1.41810 \\
\hline C & -14.94810 & 6.16940 & 0.67350 \\
\hline C & -14.73080 & 4.99370 & -0.03760 \\
\hline $\mathrm{H}$ & -14.11940 & 7.68080 & 1.92580 \\
\hline $\mathrm{H}$ & -15.92340 & 6.63950 & 0.61110 \\
\hline C & -10.06780 & 4.86850 & 1.54680 \\
\hline C & -8.79690 & 4.23380 & 1.53800 \\
\hline
\end{tabular}

$\begin{array}{lccc}\mathrm{C} & -15.80980 & 4.43810 & -0.89460 \\ \mathrm{O} & -16.92390 & 4.94110 & -0.94710 \\ \mathrm{O} & -14.02850 & 1.72250 & -2.41480 \\ \mathrm{O} & -5.44300 & 6.72250 & 2.38550 \\ \mathrm{O} & -8.10220 & 10.01410 & 4.11530 \\ \mathrm{O} & -2.02030 & 6.88230 & -2.99610 \\ \mathrm{O} & 0.28420 & 10.74390 & -3.81690 \\ \mathrm{O} & 6.47040 & 2.52400 & 2.59840 \\ \mathrm{O} & 8.89650 & 6.37370 & 2.16560 \\ \mathrm{C} & -2.10900 & 9.38350 & -3.93670 \\ \mathrm{C} & 8.62680 & 3.90850 & 3.36790 \\ \mathrm{C} & -5.55420 & 9.08730 & 3.62700 \\ \mathrm{C} & -16.49320 & 2.74960 & -2.56550 \\ \mathrm{H} & -1.88580 & 10.39160 & -4.27730 \\ \mathrm{H} & -2.49580 & 8.77500 & -4.75670 \\ \mathrm{H} & -2.85870 & 9.40350 & -3.14260 \\ \mathrm{H} & 9.42450 & 4.63680 & 3.49070 \\ \mathrm{H} & 8.10490 & 3.74870 & 4.31420 \\ \mathrm{H} & 9.02640 & 2.94960 & 3.03220 \\ \mathrm{H} & -5.85800 & 10.00750 & 4.11980 \\ \mathrm{H} & -4.93890 & 8.47800 & 4.29240 \\ \mathrm{H} & -4.97320 & 9.30550 & 2.72830 \\ \mathrm{H} & -17.40910 & 3.31970 & -2.43160 \\ \mathrm{H} & -16.14820 & 2.81520 & -3.59990 \\ \mathrm{H} & -16.65440 & 1.69650 & -2.32590\end{array}$



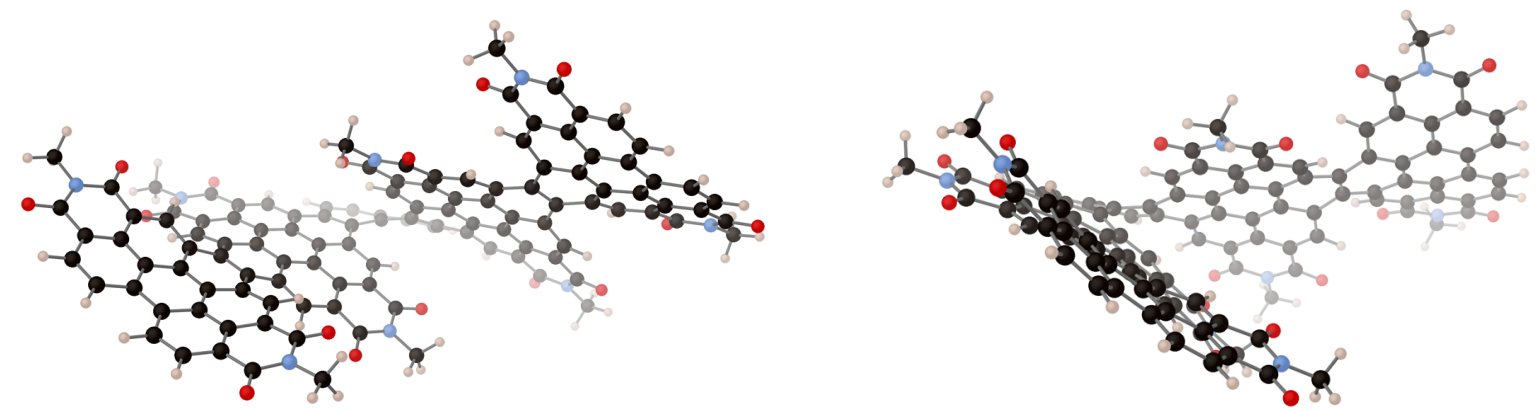

Figure S24. DFT-optimized (B3LYP/6-31G**) structure of $P M-M[6]-M P$ from different perspectives $\left(\Delta \mathrm{G}_{298.15 \mathrm{~K}}=-6166.6278\right.$ hartrees $)$.

Cartesian Coordinates of $\boldsymbol{P M}-\boldsymbol{M}[\mathbf{6}]-\mathbf{M P}$ (from left to right: atom; $\mathrm{x}, \mathrm{y}, \mathrm{z}$ in $\AA$ )

\begin{tabular}{|c|c|c|c|}
\hline C & 0.61550 & 0.87100 & -1.53240 \\
\hline C & -0.57050 & 0.07740 & -1.60140 \\
\hline C & -1.63040 & 0.48150 & -2.44430 \\
\hline C & -1.44040 & 1.58870 & -3.30220 \\
\hline C & -0.28570 & 2.34100 & -3.23210 \\
\hline C & 0.71990 & 2.09220 & -2.25910 \\
\hline C & -0.68940 & -1.14480 & -0.87080 \\
\hline C & -1.92010 & -1.85430 & -0.84760 \\
\hline C & -2.96110 & -1.40500 & -1.70160 \\
\hline C & -2.81620 & -0.28950 & -2.49690 \\
\hline C & 1.74820 & 0.35830 & -0.83180 \\
\hline C & 1.68760 & -0.94570 & -0.25690 \\
\hline C & 0.46740 & -1.67990 & -0.22540 \\
\hline C & 2.96210 & 1.09040 & -0.81940 \\
\hline C & 4.14210 & 0.40080 & -0.43700 \\
\hline C & 4.10060 & -0.86660 & 0.09970 \\
\hline C & 2.86840 & -1.54360 & 0.24160 \\
\hline C & 2.81820 & -2.84220 & 0.80000 \\
\hline C & 1.62730 & -3.53070 & 0.85470 \\
\hline C & 0.42920 & -2.98100 & 0.33390 \\
\hline C & -3.89910 & 0.07960 & -3.44500 \\
\hline$N$ & -3.64940 & 1.16570 & -4.29490 \\
\hline C & -2.47270 & 1.93780 & -4.31540 \\
\hline C & 5.37290 & -1.51980 & 0.50300 \\
\hline$N$ & 5.25630 & -2.78160 & 1.11400 \\
\hline C & 4.06030 & -3.48640 & 1.30110 \\
\hline $\mathrm{O}$ & -4.96150 & -0.52490 & -3.51250 \\
\hline $\mathrm{O}$ & -2.32020 & 2.84380 & -5.12130 \\
\hline $\mathrm{O}$ & 4.06910 & -4.58740 & 1.83520 \\
\hline $\mathrm{O}$ & 6.46270 & -0.99480 & 0.32870 \\
\hline C & -4.71620 & 1.49690 & -5.24510 \\
\hline C & 6.47560 & -3.44760 & 1.58450 \\
\hline C & -2.02610 & -3.08000 & -0.06430 \\
\hline C & -0.82380 & -3.71240 & 0.34790 \\
\hline C & -0.85220 & -5.09000 & 0.71750 \\
\hline C & -2.00660 & -5.81040 & 0.62380 \\
\hline C & -3.24310 & -5.17070 & 0.34270 \\
\hline C & -3.28440 & -3.75710 & 0.18320 \\
\hline C & -4.43120 & -5.93870 & 0.19560 \\
\hline C & -5.61370 & -5.32970 & -0.11110 \\
\hline C & -5.72310 & -3.90940 & -0.07590 \\
\hline C & -4.58690 & -3.12330 & 0.24820 \\
\hline C & -6.98950 & -3.26030 & -0.35280 \\
\hline C & -7.16270 & -1.90210 & 0.00050 \\
\hline C & -6.08730 & -1.16950 & 0.58530 \\
\hline C & -4.79880 & -1.75620 & 0.70380 \\
\hline C & -8.03270 & -3.92350 & -1.04630 \\
\hline C & -9.18770 & -3.25840 & -1.39900 \\
\hline
\end{tabular}

\begin{tabular}{|c|c|c|c|}
\hline $\mathrm{C}$ & -9.55320 & 3.82810 & 2.13640 \\
\hline$C$ & -8.71640 & 4.41800 & 3.12960 \\
\hline$C$ & -9.00060 & 5.63710 & 3.69000 \\
\hline $\mathrm{H}$ & -7.84770 & 3.90210 & 3.50970 \\
\hline$C$ & -10.17520 & 6.35000 & 3.32200 \\
\hline$C$ & -11.12270 & 5.72200 & 2.47000 \\
\hline$C$ & -12.37020 & 6.36690 & 2.21560 \\
\hline$C$ & -12.56450 & 7.65760 & 2.72930 \\
\hline $\mathrm{C}$ & -11.61360 & 8.27980 & 3.53480 \\
\hline$C$ & -10.43300 & 7.62260 & 3.86300 \\
\hline $\mathrm{H}$ & -13.49090 & 8.18290 & 2.53520 \\
\hline $\mathrm{H}$ & -11.79050 & 9.26650 & 3.94840 \\
\hline$C$ & 2.95710 & 2.47290 & -1.26320 \\
\hline$C$ & 1.85960 & 2.97180 & -2.01250 \\
\hline$C$ & 3.16410 & 5.03320 & -2.48700 \\
\hline$C$ & 1.92430 & 4.33590 & -2.51230 \\
\hline$C$ & 0.79240 & 5.05830 & -2.99390 \\
\hline$C$ & 0.91940 & 6.25840 & -3.64690 \\
\hline $\mathrm{H}$ & -0.21270 & 4.69690 & -2.83940 \\
\hline$C$ & 3.31990 & 6.28330 & -3.16760 \\
\hline C & 2.19510 & 6.86610 & -3.81030 \\
\hline$C$ & 2.32410 & 8.07040 & -4.52580 \\
\hline$C$ & 3.57070 & 8.68020 & -4.61930 \\
\hline$C$ & 4.67170 & 8.14040 & -3.95670 \\
\hline$C$ & 4.57110 & 6.97030 & -3.18830 \\
\hline $\mathrm{H}$ & 3.65670 & 9.59950 & -5.18800 \\
\hline $\mathrm{H}$ & 5.61580 & 8.66600 & -4.02070 \\
\hline$C$ & 4.24050 & 4.54080 & -1.69130 \\
\hline$C$ & 4.07000 & 3.34630 & -0.95220 \\
\hline$C$ & 4.98500 & 3.09090 & 0.11150 \\
\hline $\mathrm{C}$ & 6.12500 & 3.83460 & 0.27540 \\
\hline $\mathrm{H}$ & 4.79540 & 2.31580 & 0.84000 \\
\hline$C$ & 5.44040 & 5.30950 & -1.56070 \\
\hline$C$ & 6.41290 & 4.91790 & -0.60120 \\
\hline$C$ & 7.61280 & 5.63940 & -0.46760 \\
\hline C & 7.85390 & 6.72810 & -1.29970 \\
\hline$C$ & 6.89290 & 7.13960 & -2.22110 \\
\hline$C$ & 5.66320 & 6.47470 & -2.35090 \\
\hline $\mathrm{H}$ & 8.79020 & 7.26440 & -1.19040 \\
\hline $\mathrm{H}$ & 7.10240 & 8.01520 & -2.82190 \\
\hline C & -0.29880 & 6.92820 & -4.16870 \\
\hline $\mathrm{N}$ & -0.10120 & 8.10160 & -4.90990 \\
\hline$C$ & 1.13970 & 8.71740 & -5.14460 \\
\hline C & 7.05090 & 3.50400 & 1.38740 \\
\hline $\mathrm{N}$ & 8.24230 & 4.23890 & 1.45080 \\
\hline C & 8.60530 & 5.27760 & 0.57510 \\
\hline$C$ & -8.07860 & 6.19350 & 4.71190 \\
\hline $\mathrm{N}$ & -8.37200 & 7.47800 & 5.18920 \\
\hline
\end{tabular}




\begin{tabular}{|c|c|c|c|}
\hline C & -9.39850 & -1.92090 & -0.98840 \\
\hline C & -8.40760 & -1.25380 & -0.23440 \\
\hline C & -10.55070 & -1.20700 & -1.38800 \\
\hline C & -10.77810 & 0.06970 & -0.92100 \\
\hline C & -9.91340 & 0.68230 & 0.02510 \\
\hline C & -8.65860 & 0.06050 & 0.25480 \\
\hline C & -10.19470 & -3.93690 & -2.25260 \\
\hline$N$ & -11.28930 & -3.16610 & -2.67100 \\
\hline C & -11.52820 & -1.82350 & -2.32170 \\
\hline $\mathrm{O}$ & -12.49170 & -1.21180 & -2.75810 \\
\hline O & -10.08720 & -5.10180 & -2.61130 \\
\hline C & -12.25090 & -3.83940 & -3.55060 \\
\hline $\mathrm{H}$ & -0.17780 & 3.12070 & -3.96990 \\
\hline $\mathrm{H}$ & -3.87960 & -1.96500 & -1.79760 \\
\hline $\mathrm{H}$ & 5.11970 & 0.83710 & -0.57730 \\
\hline $\mathrm{H}$ & 1.65200 & -4.51200 & 1.30860 \\
\hline $\mathrm{H}$ & -4.38890 & 2.35620 & -5.82500 \\
\hline $\mathrm{H}$ & -4.90830 & 0.64370 & -5.89950 \\
\hline $\mathrm{H}$ & -5.63550 & 1.72530 & -4.70210 \\
\hline $\mathrm{H}$ & 7.31390 & -2.78500 & 1.38510 \\
\hline $\mathrm{H}$ & 6.39190 & -3.65430 & 2.65330 \\
\hline $\mathrm{H}$ & 6.60570 & -4.39760 & 1.06130 \\
\hline $\mathrm{H}$ & 0.06480 & -5.59760 & 0.98280 \\
\hline $\mathrm{H}$ & -2.00220 & -6.88440 & 0.78570 \\
\hline $\mathrm{H}$ & -4.36820 & -7.01930 & 0.28080 \\
\hline $\mathrm{H}$ & -6.49740 & -5.93000 & -0.28890 \\
\hline $\mathrm{H}$ & -7.92000 & -4.94070 & -1.39750 \\
\hline $\mathrm{H}$ & -11.64300 & 0.58510 & -1.31070 \\
\hline $\mathrm{H}$ & -11.74850 & -4.17500 & -4.46050 \\
\hline $\mathrm{H}$ & -12.66290 & -4.71510 & -3.04490 \\
\hline $\mathrm{H}$ & -13.03790 & -3.12820 & -3.78840 \\
\hline C & -6.32120 & 0.17130 & 1.01720 \\
\hline C & -7.61370 & 0.77440 & 0.91340 \\
\hline C & -7.83710 & 2.10570 & 1.36510 \\
\hline C & -6.68670 & 2.87090 & 1.70200 \\
\hline C & -5.43840 & 2.29580 & 1.82300 \\
\hline C & -5.24500 & 0.92660 & 1.53300 \\
\hline C & -3.78450 & -0.99850 & 1.34470 \\
\hline C & -3.98230 & 0.31330 & 1.71500 \\
\hline C & -2.84140 & 1.08940 & 2.26880 \\
\hline$N$ & -3.07370 & 2.45700 & 2.48640 \\
\hline C & -4.28800 & 3.12770 & 2.26300 \\
\hline O & -4.36680 & 4.33340 & 2.44680 \\
\hline $\mathrm{O}$ & -1.74870 & 0.58300 & 2.48930 \\
\hline C & -1.97170 & 3.28290 & 2.99030 \\
\hline $\mathrm{H}$ & -6.74500 & 3.93590 & 1.86690 \\
\hline $\mathrm{H}$ & -2.25380 & 3.73160 & 3.94530 \\
\hline $\mathrm{H}$ & -1.10420 & 2.63930 & 3.11280 \\
\hline $\mathrm{H}$ & -1.75950 & 4.08780 & 2.28380 \\
\hline $\mathrm{H}$ & -2.81840 & -1.43060 & 1.55570 \\
\hline C & -10.23790 & 1.90020 & 0.75050 \\
\hline C & -9.20490 & 2.61730 & 1.41320 \\
\hline C & -11.85480 & 3.70480 & 1.26230 \\
\hline C & -11.60590 & 2.37740 & 0.83270 \\
\hline C & -12.74060 & 1.55770 & 0.55510 \\
\hline C & -14.00410 & 2.08060 & 0.45080 \\
\hline $\mathrm{H}$ & -12.64520 & 0.48900 & 0.43440 \\
\hline C & -14.23430 & 3.46620 & 0.67560 \\
\hline C & -13.16450 & 4.27350 & 1.14800 \\
\hline C & -13.42240 & 5.62440 & 1.52300 \\
\hline C & -14.70760 & 6.14410 & 1.29600 \\
\hline C & -15.73930 & 5.35590 & 0.78830 \\
\hline C & -15.52150 & 4.00970 & 0.50770 \\
\hline $\mathrm{H}$ & -14.92650 & 7.17480 & 1.54460 \\
\hline $\mathrm{H}$ & -16.73320 & 5.76300 & 0.63990 \\
\hline C & -10.82110 & 4.43450 & 1.92060 \\
\hline
\end{tabular}

\begin{tabular}{cccc} 
C & -9.48410 & 8.25140 & 4.81410 \\
C & -15.13420 & 1.17900 & 0.11230 \\
N & -16.38490 & 1.78350 & -0.07380 \\
C & -16.65650 & 3.15450 & 0.07630 \\
O & -17.77660 & 3.60740 & -0.11710 \\
O & -15.00760 & -0.03050 & -0.01700 \\
O & -7.10240 & 5.58790 & 5.13130 \\
O & -9.66500 & 9.37120 & 5.27280 \\
O & -1.42800 & 6.49480 & -3.99020 \\
O & 1.22840 & 9.74250 & -5.80690 \\
O & 6.81620 & 2.63460 & 2.21500 \\
O & 9.67420 & 5.86170 & 0.68980 \\
C & -1.30940 & 8.72640 & -5.45780 \\
C & 9.16440 & 3.87450 & 2.53180 \\
C & -7.43340 & 8.02700 & 6.17320 \\
C & -17.48330 & 0.88720 & -0.45060 \\
H & -1.00400 & 9.61280 & -6.00810 \\
H & -1.82320 & 8.02160 & -6.11480 \\
H & -1.98840 & 8.99290 & -4.64490 \\
H & 10.03330 & 4.52410 & 2.46090 \\
H & 8.66820 & 3.99810 & 3.49670 \\
H & 9.45740 & 2.82730 & 2.43030 \\
H & -7.77450 & 9.02500 & 6.43670 \\
H & -7.40630 & 7.38440 & 7.05610 \\
H & -6.42950 & 8.06190 & 5.74550 \\
H & -18.38750 & 1.48530 & -0.53100 \\
H & -17.25660 & 0.40370 & -1.40350 \\
H & -17.60000 & 0.11010 & 0.30730 \\
& & & \\
\hline
\end{tabular}



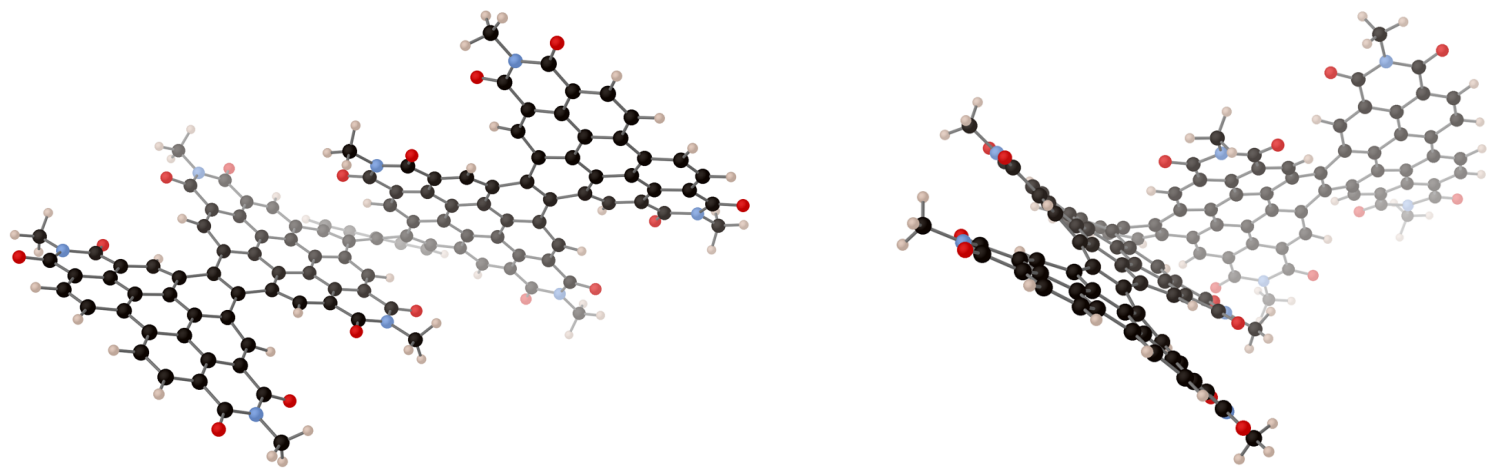

Figure S25. DFT-optimized (B3LYP/6-31G ${ }^{\star *}$ ) structure of $M$ P-M[6]-MP from different perspectives $\left(\Delta \mathrm{G}_{298.15 \mathrm{~K}}=-6166.6235\right.$ hartrees $)$.

Cartesian Coordinates of $\boldsymbol{M P}-\boldsymbol{M}[\mathbf{6}]-\mathbf{M P}$ (from left to right: atom; $\mathrm{x}, \mathrm{y}, \mathrm{z}$ in $\AA$ )

\begin{tabular}{|c|c|c|c|}
\hline C & 0.66430 & 1.10410 & -1.89390 \\
\hline C & -0.59620 & 0.42840 & -1.91020 \\
\hline C & -1.68920 & 1.02030 & -2.58090 \\
\hline C & -1.55230 & 2.32310 & -3.11260 \\
\hline C & -0.34010 & 2.98010 & -3.05720 \\
\hline C & 0.83420 & 2.34730 & -2.56810 \\
\hline C & -0.78170 & -0.82370 & -1.24770 \\
\hline C & -2.04080 & -1.48680 & -1.27790 \\
\hline C & -3.07030 & -0.90670 & -2.06260 \\
\hline C & -2.91640 & 0.32060 & -2.67000 \\
\hline C & 1.72450 & 0.57410 & -1.09990 \\
\hline C & 1.51500 & -0.63340 & -0.37190 \\
\hline C & 0.30760 & -1.37460 & -0.50830 \\
\hline C & 2.95070 & 1.27950 & -0.97960 \\
\hline C & 3.81800 & 0.89240 & 0.07840 \\
\hline C & 3.63150 & -0.27970 & 0.77920 \\
\hline C & 2.51430 & -1.10300 & 0.51100 \\
\hline C & 2.34840 & -2.34380 & 1.16940 \\
\hline C & 1.23310 & -3.11630 & 0.92600 \\
\hline C & 0.18780 & -2.64860 & 0.09150 \\
\hline C & -4.06480 & 0.92650 & -3.39220 \\
\hline$N$ & -3.88430 & 2.22790 & -3.88110 \\
\hline C & -2.71350 & 2.99880 & -3.75170 \\
\hline C & 4.60920 & -0.66040 & 1.83350 \\
\hline$N$ & 4.41130 & -1.91580 & 2.43770 \\
\hline C & 3.36210 & -2.80150 & 2.15530 \\
\hline $\mathrm{O}$ & -5.13540 & 0.35280 & -3.54500 \\
\hline $\mathrm{O}$ & -2.65900 & 4.14860 & -4.16210 \\
\hline $\mathrm{O}$ & 3.29530 & -3.88210 & 2.72550 \\
\hline $\mathrm{O}$ & 5.53840 & 0.06430 & 2.15700 \\
\hline C & -5.03390 & 2.81860 & -4.57390 \\
\hline C & 5.37170 & -2.36140 & 3.45330 \\
\hline C & -2.19990 & -2.76060 & -0.58300 \\
\hline C & -1.02980 & -3.40700 & -0.10540 \\
\hline C & -1.04310 & -4.80950 & 0.13560 \\
\hline C & -2.16210 & -5.54200 & -0.12910 \\
\hline C & -3.39150 & -4.89760 & -0.43240 \\
\hline C & -3.46140 & -3.47420 & -0.46350 \\
\hline C & -4.55210 & -5.67270 & -0.69580 \\
\hline C & -5.74630 & -5.06810 & -0.95320 \\
\hline C & -5.89940 & -3.66690 & -0.75360 \\
\hline C & -4.78520 & -2.88300 & -0.35210 \\
\hline C & -7.20640 & -3.05830 & -0.89190 \\
\hline C & -7.43440 & -1.78370 & -0.32710 \\
\hline C & -6.36850 & -1.08160 & 0.31310 \\
\hline C & -5.04390 & -1.60030 & 0.29180 \\
\hline
\end{tabular}

\begin{tabular}{|c|c|c|c|}
\hline $\mathrm{H}$ & -17.30090 & 5.20140 & 1.94720 \\
\hline C & -11.30020 & 3.93150 & 2.76670 \\
\hline C & -9.99880 & 3.36400 & 2.81480 \\
\hline C & -9.12360 & 3.83580 & 3.83870 \\
\hline $\mathrm{C}$ & -9.41730 & 4.94540 & 4.59080 \\
\hline $\mathrm{H}$ & -8.20530 & 3.31860 & 4.07390 \\
\hline C & -10.63930 & 5.64900 & 4.40000 \\
\hline C & -11.61510 & 5.10830 & 3.52000 \\
\hline C & -12.89590 & 5.73070 & 3.42810 \\
\hline C & -13.10600 & 6.92840 & 4.12860 \\
\hline C & -12.12740 & 7.46870 & 4.96040 \\
\hline C & -10.90700 & 6.82200 & 5.12840 \\
\hline $\mathrm{H}$ & -14.05870 & 7.43910 & 4.05640 \\
\hline $\mathrm{H}$ & -12.30980 & 8.38030 & 5.51700 \\
\hline C & 3.22880 & 2.36810 & -1.90260 \\
\hline C & 2.17630 & 2.90920 & -2.69030 \\
\hline C & 3.70220 & 4.70920 & -3.46580 \\
\hline C & 2.47380 & 4.00470 & -3.59990 \\
\hline C & 1.61870 & 4.40050 & -4.67220 \\
\hline C & 1.82550 & 5.56260 & -5.37350 \\
\hline $\mathrm{H}$ & 0.78240 & 3.78970 & -4.97980 \\
\hline C & 3.93350 & 5.92870 & -4.18120 \\
\hline C & 2.95020 & 6.38950 & -5.09710 \\
\hline C & 3.12390 & 7.60880 & -5.77710 \\
\hline C & 4.25990 & 8.37410 & -5.53260 \\
\hline C & 5.25130 & 7.91170 & -4.66910 \\
\hline C & 5.13680 & 6.67800 & -4.01000 \\
\hline $\mathrm{H}$ & 4.36870 & 9.31940 & -6.05290 \\
\hline $\mathrm{H}$ & 6.13890 & 8.51790 & -4.53400 \\
\hline C & 4.75960 & 4.14950 & -2.68620 \\
\hline C & 4.57120 & 2.89890 & -2.04560 \\
\hline C & 5.74120 & 2.20310 & -1.61610 \\
\hline C & 6.97070 & 2.80970 & -1.57650 \\
\hline $\mathrm{H}$ & 5.70080 & 1.16510 & -1.32050 \\
\hline C & 6.03160 & 4.80650 & -2.64020 \\
\hline C & 7.13090 & 4.15060 & -2.02230 \\
\hline C & 8.37960 & 4.78910 & -1.91610 \\
\hline C & 8.52930 & 6.08180 & -2.40690 \\
\hline C & 7.47000 & 6.71730 & -3.05170 \\
\hline C & 6.22250 & 6.09610 & -3.21650 \\
\hline $\mathrm{H}$ & 9.49370 & 6.56700 & -2.30400 \\
\hline $\mathrm{H}$ & 7.63790 & 7.70890 & -3.45270 \\
\hline 0 & 0.86130 & 5.94680 & -6.43870 \\
\hline $\mathrm{N}$ & 1.06370 & 7.19260 & -7.04800 \\
\hline C & 2.12700 & 8.07020 & -6.77630 \\
\hline & 8.13890 & 2.04560 & -1.06380 \\
\hline
\end{tabular}




\begin{tabular}{|c|c|c|c|c|c|c|c|}
\hline C & -8.24420 & -3.67680 & -1.63440 & $\mathrm{~N}$ & 9.34360 & 2.75030 & -0.94020 \\
\hline C & -9.45620 & -3.04840 & -1.82360 & $\mathrm{C}$ & 9.54480 & 4.08920 & -1.31640 \\
\hline C & -9.72200 & -1.80830 & -1.19660 & C & -8.44880 & 5.39780 & 5.62450 \\
\hline C & -8.72890 & -1.19480 & -0.39840 & $\mathrm{~N}$ & -8.76020 & 6.58580 & 6.29890 \\
\hline C & -10.94310 & -1.12960 & -1.41030 & C & -9.92090 & 7.35380 & 6.10320 \\
\hline C & -11.21840 & 0.04030 & -0.73270 & $C$ & -15.58030 & 0.82900 & 0.62410 \\
\hline C & -10.33590 & 0.56360 & 0.25230 & $\mathrm{~N}$ & -16.85530 & 1.40810 & 0.57850 \\
\hline C & -9.03030 & 0.00490 & 0.30980 & C & -17.16500 & 2.72430 & 0.96180 \\
\hline C & -10.47510 & -3.66170 & -2.71470 & $\mathrm{O}$ & -18.30540 & 3.16020 & 0.88070 \\
\hline $\mathrm{N}$ & -11.64220 & -2.91960 & -2.94220 & $\mathrm{O}$ & -15.41950 & -0.33770 & 0.29480 \\
\hline C & -11.94140 & -1.66710 & -2.37450 & $\mathrm{O}$ & -7.42100 & 4.78960 & 5.88800 \\
\hline O & -12.96950 & -1.06910 & -2.65440 & $\mathrm{O}$ & -10.11140 & 8.39040 & 6.72460 \\
\hline $\mathrm{O}$ & -10.32070 & -4.75050 & -3.25140 & $\mathrm{O}$ & -0.07830 & 5.24050 & -6.77530 \\
\hline C & -12.61980 & -3.52490 & -3.85350 & $\mathrm{O}$ & 2.22930 & 9.14530 & -7.35140 \\
\hline $\mathrm{H}$ & -0.32570 & 3.99870 & -3.41290 & $\mathrm{O}$ & 8.07660 & 0.86700 & -0.74400 \\
\hline $\mathrm{H}$ & -4.01870 & -1.40760 & -2.19010 & $\mathrm{O}$ & 10.63020 & 4.63550 & -1.17310 \\
\hline $\mathrm{H}$ & 4.65510 & 1.50610 & 0.37480 & C & 0.07380 & 7.58160 & -8.05790 \\
\hline $\mathrm{H}$ & 1.15420 & -4.05770 & 1.45560 & C & 10.47200 & 2.00120 & -0.37620 \\
\hline $\mathrm{H}$ & -4.74440 & 3.81140 & -4.90930 & C & -7.77760 & 7.03860 & 7.28910 \\
\hline $\mathrm{H}$ & -5.31310 & 2.19070 & -5.42250 & $C$ & -17.93600 & 0.54520 & 0.08900 \\
\hline $\mathrm{H}$ & -5.88800 & 2.87740 & -3.89580 & $\mathrm{H}$ & 0.33900 & 8.57270 & -8.41730 \\
\hline $\mathrm{H}$ & 6.10910 & -1.57300 & 3.58080 & $\mathrm{H}$ & 0.07940 & 6.86130 & -8.87890 \\
\hline $\mathrm{H}$ & 4.85080 & -2.55750 & 4.39280 & $\mathrm{H}$ & -0.92310 & 7.58510 & -7.61280 \\
\hline $\mathrm{H}$ & 5.85160 & -3.28700 & 3.12820 & $\mathrm{H}$ & 11.31820 & 2.67990 & -0.30460 \\
\hline $\mathrm{H}$ & -0.12960 & -5.30940 & 0.43430 & $\mathrm{H}$ & 10.20080 & 1.61550 & 0.60860 \\
\hline $\mathrm{H}$ & -2.14080 & -6.62660 & -0.07600 & $\mathrm{H}$ & 10.71410 & 1.15320 & -1.02060 \\
\hline $\mathrm{H}$ & -4.46400 & -6.75520 & -0.70770 & $\mathrm{H}$ & -8.14730 & 7.96390 & 7.72380 \\
\hline $\mathrm{H}$ & -6.60940 & -5.67030 & -1.20890 & $\mathrm{H}$ & -7.65120 & 6.27450 & 8.05900 \\
\hline $\mathrm{H}$ & -8.09120 & -4.62410 & -2.13660 & $\mathrm{H}$ & -6.81220 & 7.19910 & 6.80470 \\
\hline $\mathrm{H}$ & -12.13880 & 0.54220 & -0.98910 & $\mathrm{H}$ & -18.85880 & 1.11900 & 0.11990 \\
\hline $\mathrm{H}$ & -12.16970 & -3.66880 & -4.83820 & $\mathrm{H}$ & -17.71780 & 0.22250 & -0.93140 \\
\hline $\mathrm{H}$ & -12.92390 & -4.50110 & -3.47030 & $\mathrm{H}$ & -18.01230 & -0.34320 & 0.71910 \\
\hline
\end{tabular}


Table S2. Conformational Ensemble of $\boldsymbol{M - W H [ 6 ]}$ at $298.15 \mathrm{~K}^{\dagger}$

$\begin{array}{ccc}\text { Conformation of } \boldsymbol{M}-\mathbf{W H}[6] & \Delta \mathrm{G}(\mathrm{kcal} / \mathrm{mol}) & \text { Boltzmann Distribution (\%) } \\ \text { MM-M[6]-MM } & 2.7 & \sim 0.9 \\ \text { MM-M[6]-PP } & 0.0 & \sim 82.3 \\ \boldsymbol{P P}-M[6]-P P & 0.9 & \sim 16.8 \\ \boldsymbol{P P}-M[6]-P M & 5.6 & <0.1 \\ \text { MM-M[6]-MP } & 6.1 & <0.1 \\ \text { MM-M[6]-PM } & 6.9 & <0.1 \\ \boldsymbol{P P}-M[6]-M P & 7.4 & <0.1 \\ \boldsymbol{P M}-M[6]-P M & 10.6 & <<0.1 \\ \boldsymbol{P M - M [ 6 ] - M P} & 13.3 & <<0.1 \\ \text { MP-M[6]-MP } & 16.0 & <<0.1\end{array}$

${ }^{\dagger}$ As calculated using the Gibbs free energies corresponding to the DFT-optimized structures in Figures S16-S25.

\section{c. $\mathrm{WH}[6][6]$}
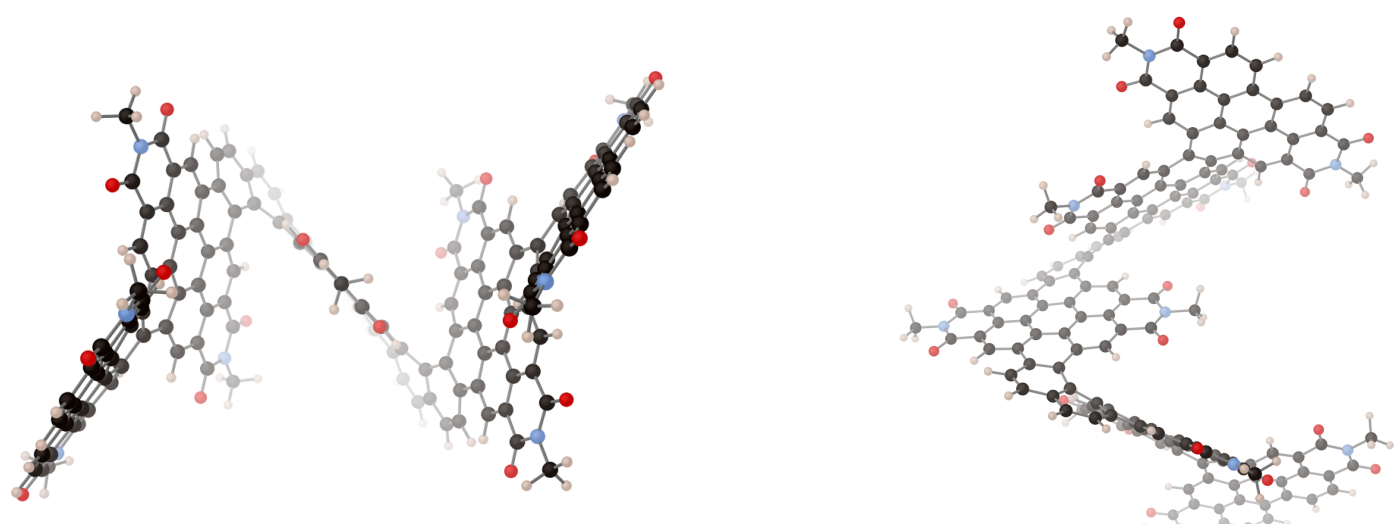

Figure S26. DFT-optimized (B3LYP/6-31G**) structure of $\mathbf{M M - M [ 6 ] [ 6 ] - M M}$ from different perspectives (total energy $=-7958.7932$ hartrees).

Cartesian Coordinates of $\mathbf{M M - M [ 6 ] [ 6 ] - M M}$ (from left to right: atom; x, y, z in $\AA$ )

$\begin{array}{llllllllllll}\mathrm{C} & 0.74390 & 1.90480 & -1.89550 & \mathrm{C} & -2.76410 & 1.01660 & 2.49260 & \mathrm{C} & -6.71700 & -1.41230 & 12.98820 \\ \mathrm{C} & -0.40040 & 1.05190 & -1.93920 & \mathrm{~N} & -3.18290 & 2.17580 & 3.16320 & \mathrm{H} & -7.46430 & -0.86660 & 11.10950 \\ \mathrm{C} & -1.57980 & 1.50530 & -2.57300 & \mathrm{C} & -4.49590 & 2.67890 & 3.18700 & \mathrm{C} & -7.65350 & -2.56710 & 13.07080 \\ \mathrm{C} & -1.61880 & 2.80560 & -3.12610 & \mathrm{O} & -4.77430 & 3.70430 & 3.79500 & \mathrm{C} & -5.83640 & -1.12050 & 14.06890 \\ \mathrm{C} & -0.50170 & 3.61290 & -3.10060 & \mathrm{O} & -1.59000 & 0.67180 & 2.53660 & \mathrm{C} & -5.02980 & 0.04980 & 14.01240 \\ \mathrm{C} & 0.72560 & 3.17430 & -2.53610 & \mathrm{C} & -2.14120 & 2.90710 & 3.88990 & \mathrm{C} & -4.16370 & 0.35830 & 15.10540 \\ \mathrm{C} & -0.37140 & -0.25580 & -1.36440 & \mathrm{H} & -7.05460 & 3.19700 & 3.02680 & \mathrm{C} & -4.14000 & -0.51580 & 16.20450 \\ \mathrm{C} & -1.53070 & -1.07620 & -1.37740 & \mathrm{H} & -2.60910 & 3.76430 & 4.36790 & \mathrm{C} & -4.94620 & -1.65160 & 16.25620 \\ \mathrm{C} & -2.67280 & -0.59960 & -2.07250 & \mathrm{H} & -1.68200 & 2.25320 & 4.63420 & \mathrm{C} & -5.79790 & -1.95860 & 15.19970 \\ \mathrm{C} & -2.70740 & 0.65180 & -2.64590 & \mathrm{H} & -1.36310 & 3.23300 & 3.19620 & \mathrm{H} & -3.49090 & -0.30960 & 17.04670 \\ \mathrm{C} & 1.92210 & 1.45740 & -1.22400 & \mathrm{H} & -2.41330 & -1.17180 & 1.09870 & \mathrm{H} & -4.93110 & -2.31070 & 17.11710 \\ \mathrm{C} & 1.98740 & 0.11910 & -0.73950 & \mathrm{C} & -9.25520 & 1.94940 & 1.90270 & \mathrm{C} & -6.67280 & -3.15440 & 15.28660 \\ \mathrm{C} & 0.85390 & -0.74230 & -0.81290 & \mathrm{C} & -10.15210 & 1.40870 & 0.94770 & \mathrm{~N} & -1.09390 & 5.94820 & 13.63670 \\ \mathrm{C} & 3.02490 & 2.33360 & -1.05710 & \mathrm{C} & -11.37930 & 2.08510 & 0.68360 & \mathrm{~N} & -7.53300 & -3.38790 & 14.20190 \\ \mathrm{C} & 4.23470 & 1.79500 & -0.54460 & \mathrm{C} & -11.65870 & 3.27390 & 1.29460 & \mathrm{O} & -0.16740 & 5.47290 & 15.68100 \\ \mathrm{C} & 4.32830 & 0.48260 & -0.13510 & \mathrm{H} & -12.04890 & 1.70790 & -0.07800 & \mathrm{O} & -1.97720 & 6.39540 & 11.58580 \\ \mathrm{C} & 3.20080 & -0.36800 & -0.19890 & \mathrm{H} & -12.52770 & 3.85550 & 1.00090 & \mathrm{O} & -6.66690 & -3.90490 & 16.25330 \\ \mathrm{C} & 3.28550 & -1.71390 & 0.23060 & \mathrm{C} & -9.74230 & 2.97430 & 2.80160 & \mathrm{O} & -8.48440 & -2.80850 & 12.20630 \\ \mathrm{C} & 2.19300 & -2.54550 & 0.13310 & \mathrm{C} & -10.84900 & 3.74970 & 2.36210 & \mathrm{C} & -0.28340 & 7.16320 & 13.50620 \\ \mathrm{C} & 0.95520 & -2.08670 & -0.38410 & \mathrm{C} & -11.13180 & 4.99040 & 2.99470 & \mathrm{C} & -8.42400 & -4.54960 & 14.29090 \\ \mathrm{C} & -3.95250 & 1.09980 & -3.32490 & \mathrm{C} & -10.36840 & 5.42950 & 4.03650 & \mathrm{H} & 0.28740 & 7.28220 & 14.42380\end{array}$




\begin{tabular}{|c|c|c|c|c|c|c|c|c|c|c|c|}
\hline$N$ & -3.94810 & 2.41410 & -3.82140 & $\mathrm{H}$ & -11.94740 & 5.59740 & 2.61250 & $\mathrm{H}$ & 0.38630 & 7.07400 & 12.64780 \\
\hline C & -2.87090 & 3.31150 & -3.74900 & $\mathrm{H}$ & -10.56630 & 6.40310 & 4.46590 & $\mathrm{H}$ & -0.93670 & 8.02230 & 13.34170 \\
\hline C & 5.63750 & -0.03420 & 0.34950 & C & -9.41540 & 4.56510 & 4.65060 & $\mathrm{H}$ & -7.91610 & -5.33090 & 14.85280 \\
\hline$N$ & 5.65510 & -1.37050 & 0.78740 & C & -9.20600 & 3.26900 & 4.11690 & $\mathrm{H}$ & -9.35130 & -4.28530 & 14.80930 \\
\hline C & 4.56280 & -2.24740 & 0.77050 & C & -8.04700 & 2.63490 & 6.22900 & $\mathrm{H}$ & -8.66290 & -4.87640 & 13.28120 \\
\hline $\mathrm{O}$ & -4.93710 & 0.38020 & -3.43070 & C & -8.61590 & 2.25500 & 4.98320 & C & 2.92770 & 3.69710 & -1.54780 \\
\hline $\mathrm{O}$ & -2.98350 & 4.44450 & -4.19470 & C & -8.71220 & 0.86840 & 4.69890 & C & 1.91480 & 4.01340 & -2.48370 \\
\hline $\mathrm{O}$ & 4.68700 & -3.39660 & 1.17280 & C & -8.21620 & -0.08690 & 5.55590 & C & 3.14540 & 6.09400 & -3.06300 \\
\hline $\mathrm{O}$ & 6.64640 & 0.65590 & 0.37180 & $\mathrm{H}$ & -9.21520 & 0.51560 & 3.81220 & C & 2.12770 & 5.14960 & -3.36100 \\
\hline C & -5.15870 & 2.91820 & -4.47770 & C & -7.51820 & 1.65080 & 7.12130 & C & 1.45220 & 5.27380 & -4.61120 \\
\hline C & 6.91320 & -1.92280 & 1.29980 & C & -7.60440 & 0.28420 & 6.77590 & C & 1.69500 & 6.31660 & -5.46780 \\
\hline C & -1.47890 & -2.40570 & -0.78510 & C & -7.09520 & -0.69380 & 7.66060 & $\mathrm{H}$ & 0.77220 & 4.51010 & -4.95850 \\
\hline C & -0.20610 & -2.94930 & -0.47070 & C & -6.57970 & -0.33280 & 8.88770 & C & 0.98530 & 6.35530 & -6.77490 \\
\hline C & -0.06240 & -4.35800 & -0.30590 & C & -6.53560 & 1.02150 & 9.30660 & C & 2.62920 & 7.33380 & -5.12680 \\
\hline C & -1.12330 & -5.18610 & -0.51820 & C & -6.93940 & 2.01700 & 8.37400 & C & 3.37050 & 7.22030 & -3.91840 \\
\hline C & -2.43040 & -4.66060 & -0.70170 & $\mathrm{H}$ & -6.17380 & -1.12450 & 9.50050 & C & 4.33090 & 8.22320 & -3.58720 \\
\hline C & -2.64820 & -3.25590 & -0.64410 & C & -8.72090 & 4.97400 & 5.85650 & C & 4.50420 & 9.29650 & -4.47550 \\
\hline C & -3.51730 & -5.54100 & -0.94350 & C & -8.04140 & 4.00420 & 6.63340 & C & 3.77790 & 9.39240 & -5.66100 \\
\hline C & -4.77870 & -5.05420 & -1.11210 & C & -8.03520 & 6.70000 & 7.43160 & C & 2.84530 & 8.41710 & -5.99800 \\
\hline C & -5.06760 & -3.68630 & -0.84080 & C & -8.69670 & 6.32320 & 6.28380 & $\mathrm{H}$ & 5.22310 & 10.07500 & -4.25200 \\
\hline C & -4.01940 & -2.81100 & -0.45330 & $\mathrm{H}$ & -9.16970 & 7.10820 & 5.70970 & $\mathrm{H}$ & 3.92910 & 10.22140 & -6.34360 \\
\hline C & -6.43850 & -3.21430 & -0.89370 & C & -7.38300 & 5.73670 & 8.23720 & C & 2.10410 & 8.52350 & -7.28040 \\
\hline C & -6.76170 & -1.96430 & -0.32020 & C & -7.39750 & 4.37750 & 7.85030 & C & 3.95390 & 5.92500 & -1.89760 \\
\hline C & -5.74500 & -1.19050 & 0.32130 & C & -6.80500 & 3.39900 & 8.70250 & C & 3.84580 & 4.73700 & -1.13390 \\
\hline C & -4.38250 & -1.59450 & 0.26540 & C & -6.10690 & 3.78670 & 9.87740 & C & 4.56240 & 4.66240 & 0.09760 \\
\hline C & -7.46300 & -3.95690 & -1.53360 & C & -6.13000 & 5.16040 & 10.23530 & C & 5.42870 & 5.64940 & 0.49300 \\
\hline C & -8.75140 & -3.47750 & -1.62210 & C & -6.75630 & 6.10470 & 9.44990 & $\mathrm{H}$ & 4.40240 & 3.84500 & 0.78590 \\
\hline C & -9.09550 & -2.23960 & -1.03010 & $\mathrm{H}$ & -5.71960 & 5.50470 & 11.17340 & C & 6.13180 & 5.51350 & 1.79780 \\
\hline C & -8.10710 & -1.49520 & -0.34360 & C & -6.79370 & 7.51800 & 9.90430 & $\mathrm{C}$ & 5.64360 & 6.79120 & -0.32960 \\
\hline C & -10.41920 & -1.74220 & -1.10770 & $\mathrm{~N}$ & -7.42090 & 8.43930 & 9.05030 & C & 4.89070 & 6.94100 & -1.52740 \\
\hline C & -10.74950 & -0.54540 & -0.50890 & $\mathrm{C}$ & -8.04360 & 8.12960 & 7.82980 & C & 5.09160 & 8.09300 & -2.34390 \\
\hline C & -9.79740 & 0.19730 & 0.23280 & C & -7.12840 & -2.13160 & 7.28990 & C & 6.03160 & 9.04560 & -1.92440 \\
\hline C & -8.47310 & -0.29460 & 0.32590 & $\mathrm{~N}$ & -7.75610 & -2.44390 & 6.06970 & C & 6.75770 & 8.89660 & -0.74520 \\
\hline C & -9.78220 & -4.27380 & -2.33750 & C & -8.33510 & -1.51920 & 5.18800 & C & 6.56980 & 7.77730 & 0.05830 \\
\hline$N$ & -11.07200 & -3.72660 & -2.40170 & $\mathrm{O}$ & -6.30920 & 7.89220 & 10.96270 & $\mathrm{H}$ & 6.21080 & 9.92900 & -2.52360 \\
\hline C & -11.47190 & -2.50820 & -1.82690 & $\mathrm{O}$ & -8.57420 & 8.99370 & 7.14570 & $\mathrm{H}$ & 7.47650 & 9.64570 & -0.43230 \\
\hline $\mathrm{O}$ & -12.62470 & -2.10860 & -1.91980 & $\mathrm{O}$ & -6.64980 & -3.00360 & 8.00100 & C & 7.33880 & 7.64150 & 1.32050 \\
\hline $\mathrm{O}$ & -9.54790 & -5.35780 & -2.85510 & $\mathrm{O}$ & -8.88350 & -1.90460 & 4.16350 & $\mathrm{~N}$ & 1.21810 & 7.47540 & -7.58210 \\
\hline C & -12.07260 & -4.52150 & -3.12140 & C & -7.42600 & 9.83430 & 9.50250 & $\mathrm{~N}$ & 7.07900 & 6.50280 & 2.09940 \\
\hline $\mathrm{H}$ & -0.61700 & 4.61280 & -3.49150 & C & -7.85110 & -3.85290 & 5.67730 & $\mathrm{O}$ & 2.25990 & 9.46500 & -8.04550 \\
\hline $\mathrm{H}$ & -3.54670 & -1.22080 & -2.19450 & $\mathrm{H}$ & -7.93620 & 10.42610 & 8.74640 & $\mathrm{O}$ & 0.22430 & 5.47340 & -7.14850 \\
\hline $\mathrm{H}$ & 5.14240 & 2.37980 & -0.51900 & $\mathrm{H}$ & -7.94160 & 9.91030 & 10.46220 & $\mathrm{O}$ & 5.91980 & 4.58860 & 2.56970 \\
\hline $\mathrm{H}$ & 2.31970 & -3.55860 & 0.49170 & $\mathrm{H}$ & -6.39990 & 10.18280 & 9.63650 & $\mathrm{O}$ & 8.15810 & 8.47470 & 1.68420 \\
\hline $\mathrm{H}$ & -4.93430 & 3.18740 & -5.51230 & $\mathrm{H}$ & -7.48980 & -3.97010 & 4.65470 & C & 0.48350 & 7.51840 & -8.85080 \\
\hline $\mathrm{H}$ & -5.90720 & 2.13050 & -4.44340 & $\mathrm{H}$ & -7.25150 & -4.43430 & 6.37300 & C & 7.82030 & 6.36730 & 3.35740 \\
\hline $\mathrm{H}$ & -5.51440 & 3.81190 & -3.96110 & $\mathrm{H}$ & -8.89260 & -4.18230 & 5.71180 & $\mathrm{H}$ & 0.72200 & 8.45880 & -9.34120 \\
\hline $\mathrm{H}$ & 7.66940 & -1.14490 & 1.23150 & C & -5.54620 & 2.76080 & 10.74600 & $\mathrm{H}$ & 0.77640 & 6.67430 & -9.47950 \\
\hline $\mathrm{H}$ & 6.78190 & -2.24150 & 2.33600 & C & -5.99340 & 1.42680 & 10.59660 & $\mathrm{H}$ & -0.58800 & 7.44300 & -8.65610 \\
\hline $\mathrm{H}$ & 7.20170 & -2.79400 & 0.70800 & C & -4.58350 & 3.05200 & 11.78810 & $\mathrm{H}$ & 8.80590 & 6.80940 & 3.22550 \\
\hline $\mathrm{H}$ & 0.91320 & -4.78400 & -0.11470 & C & -4.38220 & 2.12850 & 12.84490 & $\mathrm{H}$ & 7.30260 & 6.88950 & 4.16820 \\
\hline $\mathrm{H}$ & -0.99280 & -6.26430 & -0.51690 & C & -2.81630 & 4.46520 & 12.70620 & $\mathrm{H}$ & 7.88780 & 5.30940 & 3.60200 \\
\hline $\mathrm{H}$ & -3.31790 & -6.60550 & -1.02480 & C & -3.73160 & 4.19160 & 11.72320 & & & & \\
\hline $\mathrm{H}$ & -5.58240 & -5.73550 & -1.35660 & C & -1.95220 & 5.66470 & 12.56650 & & & & \\
\hline $\mathrm{H}$ & -7.26130 & -4.90740 & -2.00880 & $\mathrm{H}$ & -3.74920 & 4.85350 & 10.86890 & & & & \\
\hline $\mathrm{H}$ & -11.78000 & -0.22370 & -0.59080 & C & -2.69060 & 3.61500 & 13.83990 & & & & \\
\hline $\mathrm{H}$ & -11.71370 & -4.73570 & -4.12960 & $\mathrm{C}$ & -3.47050 & 2.42750 & 13.90750 & & & & \\
\hline $\mathrm{H}$ & -12.23440 & -5.47200 & -2.60750 & C & -3.33860 & 1.56490 & 15.03640 & & & & \\
\hline $\mathrm{H}$ & -12.99470 & -3.94650 & -3.15200 & C & -2.43080 & 1.92640 & 16.04410 & & & & \\
\hline C & -6.12690 & -0.01920 & 1.04370 & C & -1.66480 & 3.08760 & 15.96250 & & & & \\
\hline C & -7.48780 & 0.41550 & 1.08060 & C & -1.78340 & 3.93540 & 14.86670 & & & & \\
\hline C & -7.85960 & 1.54100 & 1.85930 & $\mathrm{H}$ & -2.30910 & 1.29620 & 16.91540 & & & & \\
\hline C & -6.83320 & 2.28220 & 2.49900 & $\mathrm{H}$ & -0.96420 & 3.35080 & 16.74730 & & & & \\
\hline C & -5.51620 & 1.88200 & 2.45660 & C & -0.94830 & 5.16150 & 14.79200 & & & & \\
\hline C & -5.14390 & 0.71490 & 1.74720 & C & -5.11400 & 0.90270 & 12.86390 & & & & \\
\hline C & -3.44140 & -0.85360 & 1.02570 & C & -5.91070 & 0.53990 & 11.74530 & & & & \\
\hline C & -3.80160 & 0.26980 & 1.73710 & $\mathrm{C}$ & -6.73790 & -0.61690 & 11.86930 & & & & \\
\hline
\end{tabular}



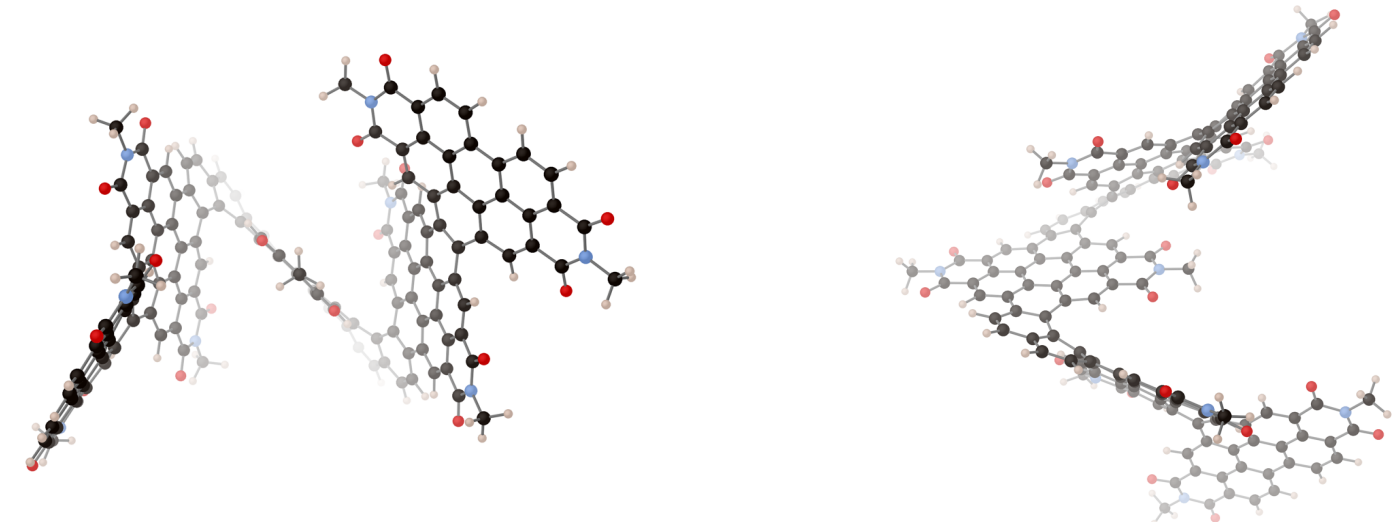

Figure S27. DFT-optimized (B3LYP/6-31G**) structure of $\mathbf{M M - M [ 6 ] [ 6 ] - P P}$ from different perspectives (total energy $=-7958.7934$ hartrees).

Cartesian Coordinates of $\mathbf{M M - M}$ 6][6]-PP (from left to right: atom; x, y, z in $\AA$ )

\begin{tabular}{|c|c|c|c|c|c|c|c|c|c|c|c|}
\hline & & & & & & & & & & & \\
\hline C & 0.74360 & 0.82620 & -2.56610 & C & -8.75640 & 4.94750 & 4.14500 & C & 5.99570 & 4.10890 & -0.61440 \\
\hline C & -0.50010 & 0.12440 & -2.55230 & C & -8.70520 & 3.60720 & 3.67930 & $\mathrm{H}$ & 4.74010 & 2.50500 & -0.10500 \\
\hline C & -1.63100 & 0.71010 & -3.16790 & C & -7.35420 & 3.01010 & 5.67800 & C & 6.73640 & 4.00100 & 0.67260 \\
\hline C & -1.52660 & 1.99780 & -3.74580 & C & -8.13570 & 2.60000 & 4.56320 & C & 6.33700 & 5.11860 & -1.56000 \\
\hline C & -0.31570 & 2.65810 & -3.77270 & C & -8.40130 & 1.21440 & 4.40940 & C & 5.56520 & 5.25380 & -2.74680 \\
\hline C & 0.86350 & 2.06640 & -3.24790 & C & -7.81420 & 0.27300 & 5.22660 & C & 5.89360 & 6.27760 & -3.68610 \\
\hline C & -0.61620 & -1.16350 & -1.94310 & $\mathrm{H}$ & -9.08860 & 0.86160 & 3.65210 & C & 6.97020 & 7.12840 & -3.39340 \\
\hline C & -1.86840 & -1.83920 & -1.90940 & C & -6.69640 & 2.04030 & 6.49400 & C & 7.71820 & 6.99060 & -2.22640 \\
\hline C & -2.95280 & -1.24670 & -2.60800 & C & -6.91950 & 0.66560 & 6.25210 & C & 7.41040 & 5.99360 & -1.30680 \\
\hline C & -2.85110 & -0.00700 & -3.20150 & C & -6.28720 & -0.29710 & 7.07400 & $\mathrm{H}$ & 7.24390 & 7.91480 & -4.08590 \\
\hline C & 1.88190 & 0.26080 & -1.91350 & $\mathrm{C}$ & -5.39240 & 0.09140 & 8.04960 & $\mathrm{H}$ & 8.54770 & 7.65510 & -2.01060 \\
\hline C & 1.78930 & -1.05740 & -1.38060 & C & -5.08990 & 1.45830 & 8.28220 & C & 8.20850 & 5.87540 & -0.06200 \\
\hline C & 0.55650 & -1.77610 & -1.40320 & C & -5.82210 & 2.43450 & 7.55210 & $\mathrm{~N}$ & 1.73860 & 5.71390 & -8.70620 \\
\hline C & 3.08980 & 1.00220 & -1.79930 & $\mathrm{H}$ & -4.97190 & -0.68790 & 8.66900 & $\mathrm{~N}$ & 7.83320 & 4.86090 & 0.83330 \\
\hline C & 4.23290 & 0.33440 & -1.28360 & C & -7.92430 & 5.36710 & 5.25610 & $\mathrm{O}$ & 3.04600 & 7.45410 & -9.42650 \\
\hline C & 4.16630 & -0.96090 & -0.81490 & C & -7.19750 & 4.39600 & 5.98570 & $\mathrm{O}$ & 0.50530 & 3.91770 & -8.04000 \\
\hline C & 2.94280 & -1.67110 & -0.83750 & C & -6.93210 & 7.12650 & 6.62030 & $\mathrm{O}$ & 6.43190 & 3.20210 & 1.54690 \\
\hline C & 2.86800 & -2.99980 & -0.35600 & C & -7.77780 & 6.73240 & 5.60670 & $\mathrm{O}$ & 9.14830 & 6.61720 & 0.19070 \\
\hline C & 1.68200 & -3.69730 & -0.41280 & $\mathrm{H}$ & -8.29470 & 7.51520 & 5.06870 & C & 0.96160 & 5.74180 & -9.94930 \\
\hline C & 0.49920 & -3.10640 & -0.92400 & C & -6.21870 & 6.16450 & 7.37300 & C & 8.60670 & 4.74190 & 2.07400 \\
\hline C & -4.04350 & 0.58210 & -3.86440 & C & -6.36090 & 4.78980 & 7.07070 & $\mathrm{H}$ & 1.19160 & 6.67050 & -10.46480 \\
\hline$N$ & -3.89140 & 1.86920 & -4.40200 & C & -5.68180 & 3.82080 & 7.86660 & $\mathrm{H}$ & 1.22520 & 4.88930 & -10.58130 \\
\hline C & -2.72460 & 2.65220 & -4.33810 & C & -4.91400 & 4.22450 & 8.99280 & $\mathrm{H}$ & -0.09940 & 5.67550 & -9.70860 \\
\hline C & 5.40220 & -1.61150 & -0.30080 & $\mathrm{C}$ & -4.71170 & 5.61610 & 9.19240 & $\mathrm{H}$ & 9.63310 & 5.03820 & 1.86760 \\
\hline$N$ & 5.25330 & -2.90560 & 0.22830 & $\mathrm{C}$ & -5.34740 & 6.55860 & 8.41370 & $\mathrm{H}$ & 8.19770 & 5.39750 & 2.84940 \\
\hline C & 4.07410 & -3.66420 & 0.20390 & $\mathrm{H}$ & -4.02560 & 5.98430 & 9.94110 & $\mathrm{H}$ & 8.54920 & 3.71050 & 2.41500 \\
\hline $\mathrm{O}$ & -5.12160 & 0.00640 & -3.93450 & C & -5.10630 & 8.00180 & 8.67420 & & & & \\
\hline $\mathrm{O}$ & -2.70550 & 3.79740 & -4.76590 & $\mathrm{~N}$ & -5.85740 & 8.91400 & 7.91760 & & & & \\
\hline $\mathrm{O}$ & 4.06170 & -4.81390 & 0.62350 & C & -6.76960 & 8.57690 & 6.90440 & & & & \\
\hline $\mathrm{O}$ & 6.49350 & -1.06080 & -0.31840 & C & -6.59430 & -1.74340 & 6.91090 & & & & \\
\hline C & -5.08030 & 2.45390 & -5.02960 & $\mathrm{~N}$ & -7.47680 & -2.07600 & 5.86720 & & & & \\
\hline C & 6.44690 & -3.55620 & 0.77930 & C & -8.11750 & -1.16410 & 5.01630 & & & & \\
\hline C & -1.96690 & -3.14970 & -1.27080 & $\mathrm{O}$ & -4.30830 & 8.40240 & 9.50830 & & & & \\
\hline C & -0.75610 & -3.83170 & -0.97860 & $\mathrm{O}$ & -7.38690 & 9.43520 & 6.28930 & & & & \\
\hline C & -0.76470 & -5.24850 & -0.81190 & $\mathrm{O}$ & -6.10980 & -2.60250 & 7.63380 & & & & \\
\hline C & -1.91640 & -5.95690 & -0.98110 & $\mathrm{O}$ & -8.88570 & -1.56230 & 4.15120 & & & & \\
\hline C & -3.16210 & -5.29090 & -1.12620 & $\mathrm{C}$ & -5.63610 & 10.33310 & 8.21610 & & & & \\
\hline C & -3.21760 & -3.86860 & -1.07920 & C & -7.79120 & -3.49040 & 5.64440 & & & & \\
\hline C & -4.35020 & -6.04400 & -1.32000 & $\mathrm{H}$ & -6.33640 & 10.91000 & 7.61740 & & & & \\
\hline C & -5.55590 & -5.42140 & -1.44900 & $\mathrm{H}$ & -5.79500 & 10.51160 & 9.28090 & & & & \\
\hline C & -5.67930 & -4.02680 & -1.18930 & $\mathrm{H}$ & -4.60800 & 10.61070 & 7.97170 & & & & \\
\hline C & -4.52320 & -3.27590 & -0.84960 & $\mathrm{H}$ & -7.59440 & -3.74760 & 4.60230 & & & & \\
\hline C & -6.98840 & -3.40070 & -1.18000 & $\mathrm{H}$ & -7.17000 & -4.07770 & 6.31550 & & & & \\
\hline C & -7.12940 & -2.10390 & -0.63220 & $\mathrm{H}$ & -8.84900 & -3.67490 & 5.84670 & & & & \\
\hline
\end{tabular}




\begin{tabular}{|c|c|c|c|c|c|c|c|}
\hline C & -6.00170 & -1.44180 & -0.05470 & $\mathrm{C}$ & -4.29220 & 3.20850 & 9.82670 \\
\hline C & -4.70810 & -2.02390 & -0.13050 & $\mathrm{C}$ & -4.16860 & 1.89130 & 9.32310 \\
\hline C & -8.13090 & -4.03310 & -1.73410 & C & -3.79920 & 3.48280 & 11.16170 \\
\hline C & -9.35390 & -3.39960 & -1.77620 & C & -2.90260 & 2.57750 & 11.78430 \\
\hline C & -9.51130 & -2.10730 & -1.22100 & C & -3.81760 & 4.84020 & 13.19490 \\
\hline C & -8.40570 & -1.47230 & -0.60760 & C & -4.28370 & 4.58000 & 11.93250 \\
\hline C & -10.76040 & -1.44350 & -1.27160 & C & -4.36880 & 6.00170 & 13.93840 \\
\hline C & -10.90680 & -0.19230 & -0.71390 & $\mathrm{H}$ & -5.07580 & 5.21520 & 11.56350 \\
\hline C & -9.83490 & 0.44620 & -0.04130 & C & -2.82020 & 4.01400 & 13.78450 \\
\hline C & -8.58390 & -0.20830 & 0.02080 & C & -2.37090 & 2.86020 & 13.08400 \\
\hline C & -10.51170 & -4.08400 & -2.40820 & C & -1.39560 & 2.01100 & 13.68670 \\
\hline $\mathrm{N}$ & -11.72450 & -3.37990 & -2.43580 & C & -0.90580 & 2.35850 & 14.95550 \\
\hline C & -11.93320 & -2.09030 & -1.91780 & C & -1.35330 & 3.49140 & 15.63200 \\
\hline O & -13.02390 & -1.54120 & -1.99700 & C & -2.31060 & 4.32200 & 15.05950 \\
\hline $\mathrm{O}$ & -10.44320 & -5.20810 & -2.88690 & $\mathrm{H}$ & -0.15970 & 1.73940 & 15.43630 \\
\hline C & -12.85400 & -4.06940 & -3.06820 & $\mathrm{H}$ & -0.96870 & 3.74600 & 16.61350 \\
\hline $\mathrm{H}$ & -0.30820 & 3.66030 & -4.17950 & C & -2.78950 & 5.51410 & 15.80500 \\
\hline $\mathrm{H}$ & -3.89890 & -1.76000 & -2.69660 & C & -2.50850 & 1.38650 & 11.10040 \\
\hline $\mathrm{H}$ & 5.20610 & 0.80360 & -1.28830 & C & -3.13030 & 1.04030 & 9.87190 \\
\hline $\mathrm{H}$ & 1.68870 & -4.70320 & -0.01630 & C & -2.62270 & -0.08750 & 9.16210 \\
\hline $\mathrm{H}$ & -4.76430 & 3.32820 & -5.59260 & C & -1.63410 & -0.88400 & 9.68120 \\
\hline $\mathrm{H}$ & -5.54150 & 1.71040 & -5.67940 & $\mathrm{H}$ & -2.96770 & -0.31990 & 8.16470 \\
\hline $\mathrm{H}$ & -5.80890 & 2.74790 & -4.26870 & C & -1.14270 & -2.03790 & 8.88120 \\
\hline $\mathrm{H}$ & 7.14050 & -2.78020 & 1.09430 & C & -1.08080 & -0.61300 & 10.96480 \\
\hline $\mathrm{H}$ & 6.14460 & -4.18410 & 1.61560 & C & -1.50970 & 0.53940 & 11.67880 \\
\hline $\mathrm{H}$ & 6.92820 & -4.18490 & 0.02360 & C & -0.94290 & 0.82500 & 12.95810 \\
\hline $\mathrm{H}$ & 0.16500 & -5.77800 & -0.65340 & C & 0.01830 & -0.05950 & 13.47050 \\
\hline $\mathrm{H}$ & -1.90450 & -7.04290 & -0.97380 & C & 0.44050 & -1.17850 & 12.75630 \\
\hline $\mathrm{H}$ & -4.27450 & -7.12510 & -1.38920 & C & -0.09840 & -1.46320 & 11.50650 \\
\hline $\mathrm{H}$ & -6.43900 & -6.00990 & -1.66160 & $\mathrm{H}$ & 0.45940 & 0.12360 & 14.44150 \\
\hline $\mathrm{H}$ & -8.07600 & -5.01880 & -2.17780 & $\mathrm{H}$ & 1.19260 & -1.84790 & 13.15920 \\
\hline $\mathrm{H}$ & -11.88900 & 0.25870 & -0.77310 & C & 0.37480 & -2.65690 & 10.76100 \\
\hline $\mathrm{H}$ & -12.61710 & -4.29050 & -4.11120 & $\mathrm{~N}$ & -3.78200 & 6.28700 & 15.17850 \\
\hline $\mathrm{H}$ & -13.04310 & -5.01410 & -2.55440 & $\mathrm{~N}$ & -0.18820 & -2.86270 & 9.48990 \\
\hline $\mathrm{H}$ & -13.71960 & -3.41530 & -3.00210 & $\mathrm{O}$ & -2.35360 & 5.81370 & 16.90830 \\
\hline C & -6.19680 & -0.20530 & 0.63140 & $\mathrm{O}$ & -5.27620 & 6.70000 & 13.50820 \\
\hline C & -7.48630 & 0.40550 & 0.70030 & $\mathrm{O}$ & 1.21460 & -3.42110 & 11.21720 \\
\hline C & -7.67720 & 1.59850 & 1.44630 & $\mathrm{O}$ & -1.54090 & -2.28520 & 7.75130 \\
\hline C & -6.53020 & 2.23620 & 1.98220 & C & -4.29130 & 7.47060 & 15.87910 \\
\hline C & -5.28450 & 1.65230 & 1.93210 & C & 0.25540 & -4.01950 & 8.70560 \\
\hline C & -5.09920 & 0.41320 & 1.27340 & $\mathrm{H}$ & -3.62040 & 7.68080 & 16.70810 \\
\hline C & -3.65470 & -1.40430 & 0.58790 & $\mathrm{H}$ & -4.33140 & 8.30810 & 15.18210 \\
\hline C & -3.83000 & -0.20960 & 1.24750 & $\mathrm{H}$ & -5.30160 & 7.28590 & 16.25360 \\
\hline C & -2.66620 & 0.43850 & 1.90490 & $\mathrm{H}$ & 0.92620 & -4.60440 & 9.32970 \\
\hline $\mathrm{N}$ & -2.91550 & 1.64680 & 2.57640 & $\mathrm{H}$ & -0.61210 & -4.61010 & 8.40570 \\
\hline C & -4.14400 & 2.32810 & 2.60490 & $\mathrm{H}$ & 0.76960 & -3.68370 & 7.80200 \\
\hline O & -4.25730 & 3.40510 & 3.17640 & C & 3.16100 & 2.33670 & -2.37550 \\
\hline O & -1.53370 & -0.02490 & 1.87070 & C & 2.16090 & 2.72350 & -3.29830 \\
\hline C & -1.76520 & 2.26960 & 3.23750 & C & 3.65750 & 4.51960 & -4.13580 \\
\hline $\mathrm{H}$ & -6.60630 & 3.20040 & 2.46170 & C & 2.49910 & 3.71650 & -4.29830 \\
\hline $\mathrm{H}$ & -2.14180 & 3.03070 & 3.91580 & C & 1.79120 & 3.80810 & -5.53250 \\
\hline $\mathrm{H}$ & -1.20480 & 1.50300 & 3.77180 & C & 2.14940 & 4.70660 & -6.50400 \\
\hline $\mathrm{H}$ & -1.10220 & 2.73140 & 2.50010 & $\mathrm{H}$ & 0.99440 & 3.11770 & -5.77260 \\
\hline $\mathrm{H}$ & -2.68100 & -1.86560 & 0.64910 & C & 1.39350 & 4.71830 & -7.78240 \\
\hline C & -9.00880 & 2.18120 & 1.53700 & C & 3.23260 & 5.60720 & -6.30190 \\
\hline C & -10.00460 & 1.71340 & 0.64090 & C & 4.00240 & 5.50980 & -5.11060 \\
\hline C & -11.15230 & 2.51600 & 0.38550 & C & 5.10900 & 6.38930 & -4.91620 \\
\hline C & -11.27970 & 3.74470 & 0.95950 & C & 5.39410 & 7.33000 & -5.91810 \\
\hline $\mathrm{H}$ & -11.88550 & 2.18870 & -0.33970 & C & 4.63770 & 7.41120 & -7.08520 \\
\hline $\mathrm{H}$ & -12.09160 & 4.40760 & 0.67460 & C & 3.56140 & 6.55430 & -7.28940 \\
\hline C & -9.33950 & 3.29430 & 2.40930 & $\mathrm{H}$ & 6.22460 & 8.01400 & -5.79870 \\
\hline C & -10.38180 & 4.16330 & 1.97600 & $\mathrm{H}$ & 4.87400 & 8.13680 & -7.85570 \\
\hline C & -10.52410 & 5.44650 & 2.56700 & C & 2.78750 & 6.63650 & -8.55370 \\
\hline C & -9.67410 & 5.85950 & 3.54860 & C & 4.47790 & 4.35210 & -2.97920 \\
\hline $\mathrm{H}$ & -11.30280 & 6.10660 & 2.19590 & C & 4.22950 & 3.27540 & -2.08870 \\
\hline $\mathrm{H}$ & -9.75510 & 6.86900 & 3.93080 & $\mathrm{C}$ & 4.98220 & 3.22120 & -0.87790 \\
\hline
\end{tabular}



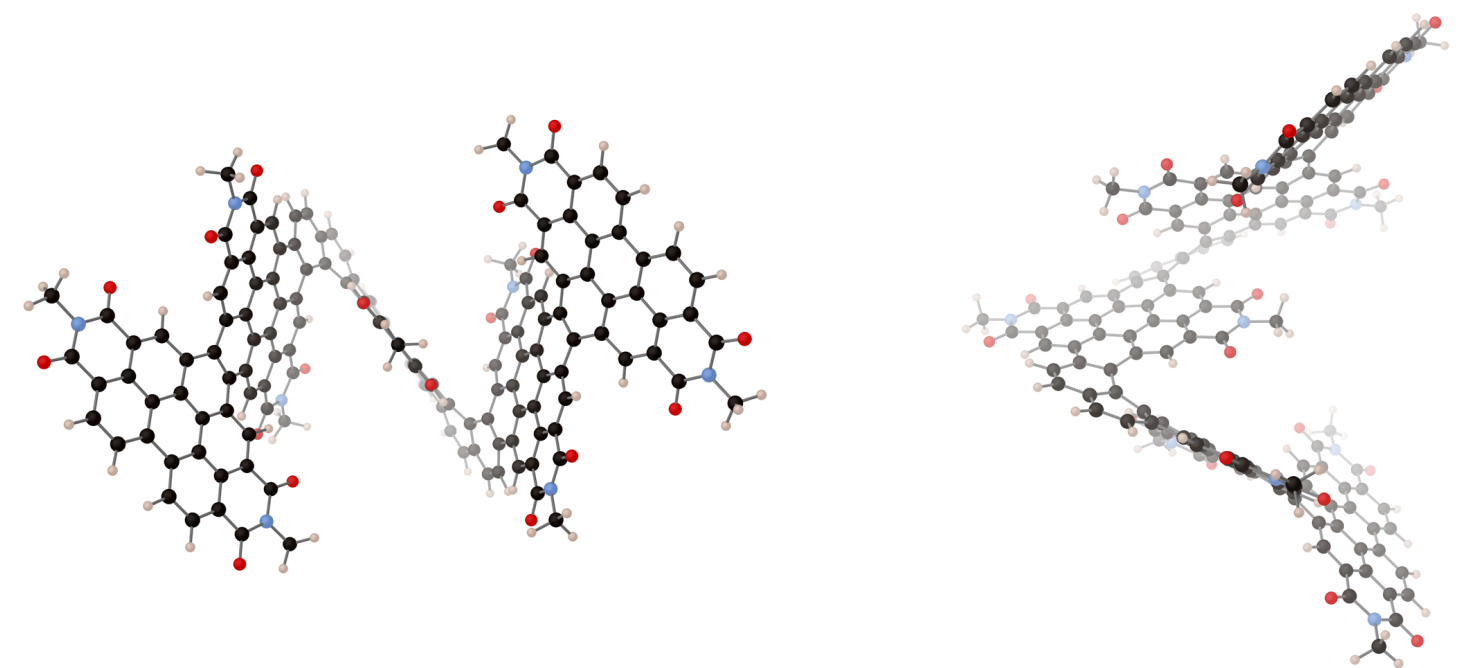

Figure S28. DFT-optimized (B3LYP/6-31G**) structure of $\boldsymbol{P P}-\boldsymbol{M}$ 6][6]-PP from different perspectives (total energy $=-7958.7940$ hartrees).

Cartesian Coordinates of PP-M[6][6]-PP (from left to right: atom; x, y, z in $\AA$ )

\begin{tabular}{|c|c|c|c|c|c|c|c|}
\hline C & 0.31660 & 1.09850 & -2.86960 & C & -10.38920 & 5.30290 & 2.65420 \\
\hline C & -0.85100 & 0.27870 & -2.83470 & $\mathrm{C}$ & -9.47120 & 5.75560 & 3.55730 \\
\hline C & -2.05510 & 0.76370 & -3.39670 & $\mathrm{H}$ & -11.18470 & 5.95240 & 2.30090 \\
\hline C & -2.07840 & 2.04070 & -4.00400 & $\mathrm{H}$ & -9.52390 & 6.78160 & 3.89850 \\
\hline C & -0.96000 & 2.84670 & -3.98300 & $\mathrm{C}$ & -8.52900 & 4.86180 & 4.14770 \\
\hline C & 0.24900 & 2.42980 & -3.36720 & C & -8.51830 & 3.50240 & 3.74400 \\
\hline C & -0.82020 & -1.02680 & -2.25510 & C & -7.13300 & 2.96760 & 5.73950 \\
\hline C & -2.00810 & -1.80010 & -2.15980 & $\mathrm{C}$ & -7.92640 & 2.52420 & 4.64610 \\
\hline C & -3.17780 & -1.29740 & -2.78640 & C & -8.21080 & 1.13690 & 4.55260 \\
\hline C & -3.20670 & -0.05910 & -3.38840 & $\mathrm{C}$ & -7.66420 & 0.22360 & 5.42630 \\
\hline C & 1.55640 & 0.57320 & -2.39230 & $\mathrm{H}$ & -8.89660 & 0.75990 & 3.80730 \\
\hline C & 1.59470 & -0.73600 & -1.83030 & C & -6.51730 & 2.02360 & 6.61730 \\
\hline C & 0.42310 & -1.54770 & -1.78040 & $\mathrm{C}$ & -6.78360 & 0.64500 & 6.45130 \\
\hline C & 2.75020 & 1.33110 & -2.52110 & C & -6.21540 & -0.28820 & 7.34750 \\
\hline C & 3.92830 & 0.83220 & -1.90490 & $\mathrm{C}$ & -5.33780 & 0.12350 & 8.32750 \\
\hline C & 3.96960 & -0.42390 & -1.33720 & C & -4.98780 & 1.48900 & 8.48640 \\
\hline C & 2.82010 & -1.24860 & -1.34200 & C & -5.65890 & 2.44640 & 7.67730 \\
\hline C & 2.87200 & -2.56750 & -0.83130 & $\mathrm{H}$ & -4.97240 & -0.63290 & 9.00660 \\
\hline C & 1.74960 & -3.36690 & -0.84440 & C & -7.63980 & 5.31670 & 5.20130 \\
\hline C & 0.50350 & -2.88360 & -1.31740 & $\mathrm{C}$ & -6.93830 & 4.36230 & 5.97850 \\
\hline C & -4.46380 & 0.41300 & -4.02450 & $\mathrm{C}$ & -6.57380 & 7.11450 & 6.45940 \\
\hline$N$ & -4.41680 & 1.66200 & -4.66040 & C & -7.43250 & 6.69330 & 5.46740 \\
\hline C & -3.30390 & 2.52100 & -4.69820 & $\mathrm{H}$ & -7.91590 & 7.46170 & 4.87940 \\
\hline C & 5.23500 & -0.89930 & -0.71380 & $\mathrm{C}$ & -5.90810 & 6.17020 & 7.27820 \\
\hline$N$ & 5.23120 & -2.21980 & -0.23010 & $\mathrm{C}$ & -6.09820 & 4.78690 & 7.05150 \\
\hline C & 4.13990 & -3.09830 & -0.26670 & $\mathrm{C}$ & -5.47440 & 3.84170 & 7.91980 \\
\hline $\mathrm{O}$ & -5.50260 & -0.23480 & -4.01460 & $\mathrm{C}$ & -4.72580 & 4.28040 & 9.04770 \\
\hline $\mathrm{O}$ & -3.35170 & 3.59880 & -5.27390 & $\mathrm{C}$ & -4.47360 & 5.67260 & 9.17190 \\
\hline $\mathrm{O}$ & 4.25130 & -4.23830 & 0.16430 & C & -5.04620 & 6.59100 & 8.31690 \\
\hline $\mathrm{O}$ & 6.22890 & -0.19250 & -0.62950 & $\mathrm{H}$ & -3.79720 & 6.05750 & 9.92250 \\
\hline C & -5.65400 & 2.09790 & -5.31480 & C & -4.74890 & 8.03570 & 8.49440 \\
\hline C & 6.46270 & -2.75150 & 0.36270 & $\mathrm{~N}$ & -5.42840 & 8.92970 & 7.65320 \\
\hline C & -1.96060 & -3.11430 & -1.53280 & C & -6.35420 & 8.57240 & 6.65940 \\
\hline C & -0.69080 & -3.70710 & -1.31630 & $\mathrm{C}$ & -6.58060 & -1.72640 & 7.26870 \\
\hline C & -0.60120 & -5.12040 & -1.14760 & $\mathrm{~N}$ & -7.45850 & -2.08940 & 6.23190 \\
\hline C & -1.71510 & -5.90400 & -1.23630 & $\mathrm{C}$ & -8.00460 & -1.21420 & 5.28280 \\
\hline C & -3.01000 & -5.31770 & -1.28130 & $\mathrm{O}$ & -3.95970 & 8.45310 & 9.32900 \\
\hline C & -3.14230 & -3.90290 & -1.24140 & $\mathrm{O}$ & -6.93280 & 9.41870 & 5.99130 \\
\hline C & -4.17360 & -6.13020 & -1.34900 & $\mathrm{O}$ & -6.14790 & -2.55520 & 8.05570 \\
\hline C & -5.41650 & -5.56760 & -1.36210 & $\mathrm{O}$ & -8.73340 & -1.64050 & 4.39710 \\
\hline
\end{tabular}

$\begin{array}{cccc}\mathrm{C} & 2.51830 & 9.53000 & -4.73820 \\ \mathrm{C} & 1.38090 & 8.88900 & -4.25910 \\ \mathrm{H} & 4.55300 & 9.34950 & -5.34220 \\ \mathrm{H} & 2.46920 & 10.59630 & -4.92960 \\ \mathrm{C} & 0.14550 & 9.67550 & -4.01300 \\ \mathrm{C} & 3.83250 & 4.61730 & -4.13000 \\ \mathrm{C} & 3.87960 & 3.25110 & -3.75320 \\ \mathrm{C} & 5.05100 & 2.50570 & -4.07770 \\ \mathrm{C} & 6.15290 & 3.09710 & -4.64040 \\ \mathrm{H} & 5.08380 & 1.43350 & -3.94810 \\ \mathrm{C} & 7.32930 & 2.24700 & -4.97170 \\ \mathrm{C} & 6.16510 & 4.49450 & -4.90890 \\ \mathrm{C} & 4.99240 & 5.26250 & -4.66610 \\ \mathrm{C} & 4.99260 & 6.65770 & -4.96640 \\ \mathrm{C} & 6.16760 & 7.22610 & -5.48340 \\ \mathrm{C} & 7.30880 & 6.46280 & -5.72320 \\ \mathrm{C} & 7.31570 & 5.10030 & -5.44610 \\ \mathrm{H} & 6.20230 & 8.28250 & -5.71640 \\ \mathrm{H} & 8.20720 & 6.91250 & -6.13160 \\ \mathrm{C} & 8.52790 & 4.29420 & -5.73420 \\ \mathrm{~N} & -0.94890 & 8.97100 & -3.48310 \\ \mathrm{~N} & 8.44940 & 2.91590 & -5.48830 \\ \mathrm{O} & 0.07770 & 10.87470 & -4.24730 \\ \mathrm{O} & -1.96520 & 7.09000 & -2.69940 \\ \mathrm{O} & 7.33050 & 1.03530 & -4.80430 \\ \mathrm{O} & 9.55800 & 4.78160 & -6.18000 \\ \mathrm{C} & -2.18770 & 9.70480 & -3.20620 \\ \mathrm{C} & 9.64850 & 2.13310 & -5.80500 \\ \mathrm{H} & -2.04070 & 10.73480 & -3.52170 \\ \mathrm{H} & -3.01500 & 9.24710 & -3.75230 \\ \mathrm{H} & -2.41600 & 9.65920 & -2.13900 \\ \mathrm{H} & 9.89770 & 2.24920 & -6.86180 \\ \mathrm{H} & 10.49240 & 2.49400 & -5.21330 \\ & 9.43550 & 1.09260 & -5.57280\end{array}$




\begin{tabular}{|c|c|c|c|c|c|c|c|}
\hline C & -5.58650 & -4.17750 & -1.10130 & $\mathrm{C}$ & -5.13630 & 10.35220 & 7.85890 \\
\hline C & -4.44220 & -3.36720 & -0.88810 & $\mathrm{C}$ & -7.82990 & -3.50080 & 6.09600 \\
\hline C & -6.91420 & -3.60270 & -1.00270 & $\mathrm{H}$ & -5.68630 & 10.91580 & 7.10940 \\
\hline C & -7.06360 & -2.29820 & -0.47510 & $\mathrm{H}$ & -5.44130 & 10.65410 & 8.86340 \\
\hline C & -5.92130 & -1.57750 & -0.00550 & $\mathrm{H}$ & -4.06250 & 10.52240 & 7.76150 \\
\hline C & -4.61450 & -2.10130 & -0.18820 & $\mathrm{H}$ & -7.39350 & -3.91850 & 5.18520 \\
\hline C & -8.06780 & -4.28900 & -1.45920 & $\mathrm{H}$ & -7.45300 & -4.02950 & 6.96790 \\
\hline C & -9.31230 & -3.69780 & -1.43300 & $\mathrm{H}$ & -8.91540 & -3.58510 & 6.02650 \\
\hline C & -9.47720 & -2.39730 & -0.90020 & C & -4.16280 & 3.29300 & 9.95790 \\
\hline C & -8.35670 & -1.70900 & -0.37820 & C & -4.08550 & 1.94520 & 9.53270 \\
\hline C & -10.74800 & -1.77370 & -0.88330 & C & -3.69240 & 3.61690 & 11.29270 \\
\hline C & -10.89990 & -0.51100 & -0.35020 & C & -2.87110 & 2.69840 & 11.99850 \\
\hline C & -9.81030 & 0.18130 & 0.23430 & C & -3.71990 & 5.06600 & 13.26300 \\
\hline C & -8.53580 & -0.43430 & 0.23150 & C & -4.14180 & 4.77630 & 11.99040 \\
\hline C & -10.48560 & -4.43490 & -1.97000 & C & -4.24750 & 6.28090 & 13.93590 \\
\hline $\mathrm{N}$ & -11.71940 & -3.76900 & -1.93810 & $\mathrm{H}$ & -4.87850 & 5.43800 & 11.55850 \\
\hline C & -11.93670 & -2.47350 & -1.43820 & C & -2.79540 & 4.21830 & 13.93440 \\
\hline O & -13.04750 & -1.96060 & -1.46190 & C & -2.37480 & 3.01500 & 13.30470 \\
\hline O & -10.41120 & -5.56880 & -2.42430 & C & -1.47140 & 2.14650 & 13.98770 \\
\hline C & -12.86280 & -4.50970 & -2.48090 & C & -1.02090 & 2.52300 & 15.26270 \\
\hline $\mathrm{H}$ & -1.02960 & 3.79220 & -4.50070 & C & -1.44280 & 3.70280 & 15.87100 \\
\hline $\mathrm{H}$ & -4.08330 & -1.88570 & -2.82120 & C & -2.33190 & 4.55170 & 15.22090 \\
\hline $\mathrm{H}$ & 4.82470 & 1.43310 & -1.83980 & $\mathrm{H}$ & -0.32980 & 1.88800 & 15.80310 \\
\hline $\mathrm{H}$ & 1.85160 & -4.36400 & -0.43470 & $\mathrm{H}$ & -1.09210 & 3.97870 & 16.85950 \\
\hline $\mathrm{H}$ & -5.43060 & 2.99580 & -5.88530 & C & -2.79190 & 5.78870 & 15.90000 \\
\hline $\mathrm{H}$ & -6.01860 & 1.30190 & -5.96570 & C & -2.51790 & 1.45410 & 11.39520 \\
\hline $\mathrm{H}$ & -6.42240 & 2.30840 & -4.56670 & C & -3.11450 & 1.07480 & 10.16520 \\
\hline $\mathrm{H}$ & 7.21160 & -1.96400 & 0.33480 & C & -2.64160 & -0.11130 & 9.53200 \\
\hline $\mathrm{H}$ & 6.27150 & -3.06540 & 1.39100 & C & -1.71990 & -0.93200 & 10.12930 \\
\hline $\mathrm{H}$ & 6.79910 & -3.62260 & -0.20360 & $\mathrm{H}$ & -2.96080 & -0.37770 & 8.53480 \\
\hline $\mathrm{H}$ & 0.37000 & -5.58670 & -1.04160 & C & -1.26930 & -2.15060 & 9.40970 \\
\hline $\mathrm{H}$ & -1.63120 & -6.98680 & -1.23310 & C & -1.19910 & -0.62400 & 11.41690 \\
\hline $\mathrm{H}$ & -4.05400 & -7.20740 & -1.41870 & C & -1.58790 & 0.58650 & 12.05310 \\
\hline $\mathrm{H}$ & -6.28600 & -6.20210 & -1.47870 & C & -1.05040 & 0.90840 & 13.33490 \\
\hline $\mathrm{H}$ & -8.00490 & -5.28050 & -1.88810 & C & -0.14860 & 0.00880 & 13.92420 \\
\hline $\mathrm{H}$ & -11.89830 & -0.09270 & -0.35630 & C & 0.22940 & -1.17180 & 13.28950 \\
\hline $\mathrm{H}$ & -12.68120 & -4.75590 & -3.52930 & C & -0.28630 & -1.49580 & 12.03950 \\
\hline $\mathrm{H}$ & -12.99330 & -5.44290 & -1.92900 & $\mathrm{H}$ & 0.27220 & 0.22220 & 14.89840 \\
\hline $\mathrm{H}$ & -13.74320 & -3.87950 & -2.38300 & $\mathrm{H}$ & 0.92890 & -1.85730 & 13.75520 \\
\hline C & -6.11240 & -0.33300 & 0.66950 & C & 0.13830 & -2.75430 & 11.37770 \\
\hline C & -7.41510 & 0.23250 & 0.81850 & $\mathrm{~N}$ & -3.73930 & 6.56480 & 15.21050 \\
\hline C & -7.59340 & 1.44320 & 1.53870 & $\mathrm{~N}$ & -0.38340 & -2.99280 & 10.09460 \\
\hline C & -6.43750 & 2.12070 & 2.00180 & $\mathrm{O}$ & -2.38470 & 6.11530 & 17.00670 \\
\hline C & -5.17630 & 1.58840 & 1.85690 & $\mathrm{O}$ & -5.08070 & 7.01790 & 13.42700 \\
\hline C & -4.99290 & 0.34950 & 1.19980 & $\mathrm{O}$ & 0.90830 & -3.54290 & 11.90940 \\
\hline C & -3.53060 & -1.41090 & 0.41190 & $\mathrm{O}$ & -1.64450 & -2.43790 & 8.28120 \\
\hline C & -3.70400 & -0.21560 & 1.07060 & C & -4.25090 & 7.78060 & 15.85130 \\
\hline C & -2.51770 & 0.49930 & 1.60700 & C & 0.01670 & -4.21520 & 9.39080 \\
\hline $\mathrm{N}$ & -2.75580 & 1.72550 & 2.24550 & $\mathrm{H}$ & -3.80770 & 7.84580 & 16.84190 \\
\hline C & -4.01550 & 2.32240 & 2.42600 & $\mathrm{H}$ & -3.98430 & 8.65580 & 15.25430 \\
\hline $\mathrm{O}$ & -4.13730 & 3.38210 & 3.02690 & $\mathrm{H}$ & -5.33950 & 7.73030 & 15.91600 \\
\hline O & -1.37430 & 0.07370 & 1.50160 & $\mathrm{H}$ & 0.67540 & -4.77650 & 10.04900 \\
\hline C & -1.57500 & 2.41450 & 2.77300 & $\mathrm{H}$ & -0.86990 & -4.80140 & 9.14010 \\
\hline $\mathrm{H}$ & -6.51350 & 3.08350 & 2.48240 & $\mathrm{H}$ & 0.53000 & -3.95770 & 8.46180 \\
\hline $\mathrm{H}$ & -1.89520 & 3.38290 & 3.14920 & C & 2.70200 & 2.61410 & -3.20060 \\
\hline $\mathrm{H}$ & -1.12400 & 1.82530 & 3.57540 & C & 1.45030 & 3.25030 & -3.37070 \\
\hline $\mathrm{H}$ & -0.83600 & 2.53130 & 1.97850 & C & 2.62100 & 5.35710 & -3.96690 \\
\hline $\mathrm{H}$ & -2.52990 & -1.81280 & 0.38020 & C & 1.43180 & 4.68530 & -3.58040 \\
\hline C & -8.93130 & 1.99710 & 1.68580 & $\mathrm{C}$ & 0.28030 & 5.47800 & -3.29940 \\
\hline C & -9.96910 & 1.47510 & 0.87300 & C & 0.26850 & 6.83520 & -3.50220 \\
\hline C & -11.14370 & 2.25330 & 0.66070 & $\mathrm{H}$ & -0.60990 & 5.03670 & -2.87320 \\
\hline C & -11.24800 & 3.50970 & 1.18410 & C & -0.96620 & 7.60240 & -3.18520 \\
\hline $\mathrm{H}$ & -11.91740 & 1.88640 & -0.00190 & C & 1.41850 & 7.50450 & -4.00850 \\
\hline $\mathrm{H}$ & -12.08310 & 4.15130 & 0.91800 & C & 2.61260 & 6.76420 & -4.23140 \\
\hline C & -9.22340 & 3.14170 & 2.52700 & C & 3.77460 & 7.43420 & -4.72070 \\
\hline C & -10.29160 & 3.98760 & 2.12080 & $\mathrm{C}$ & 3.69130 & 8.81320 & -4.96570 \\
\hline
\end{tabular}



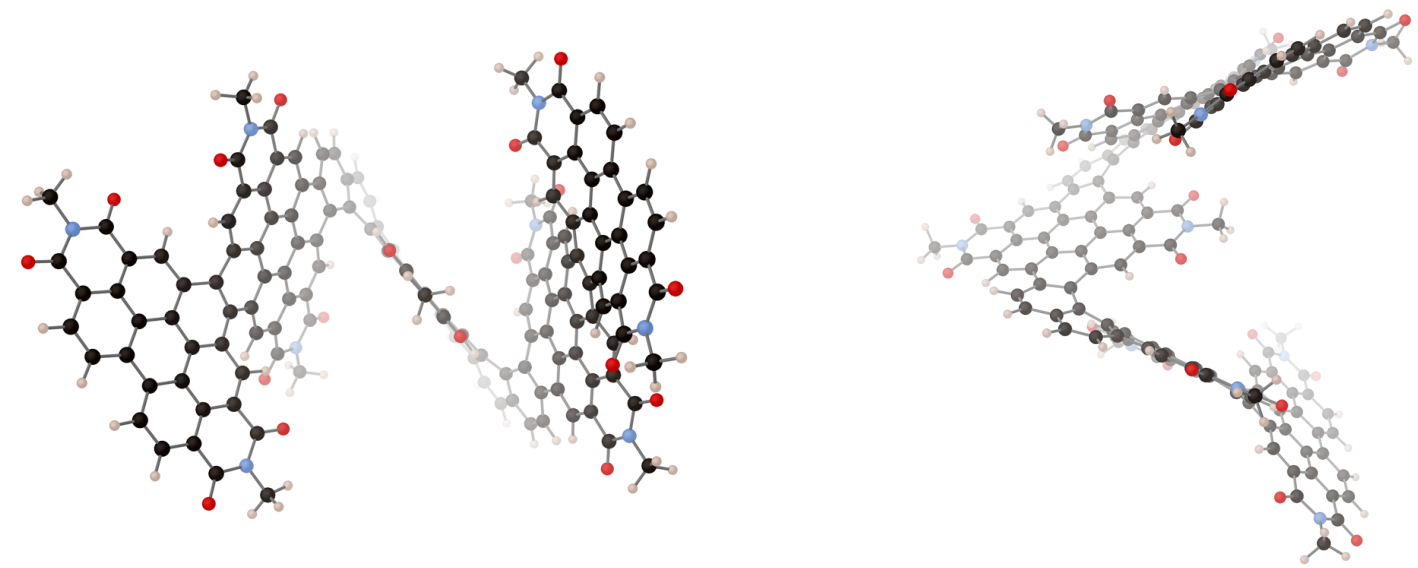

Figure S29. DFT-optimized (B3LYP/6-31G**) structure of $P \boldsymbol{P}-\boldsymbol{M}$ [6][6]-PM from different perspectives (total energy $=-7958.7838$ hartrees).

Cartesian Coordinates of PP-M[6][6]-PM (from left to right: atom; $x, y, z$ in $\AA$ )

\begin{tabular}{|c|c|c|c|c|c|c|c|}
\hline C & 0.37760 & 0.94850 & -2.87910 & $\mathrm{H}$ & -9.09450 & 6.78740 & 3.55550 \\
\hline C & -0.80960 & 0.15660 & -2.92370 & C & -8.16230 & 4.85750 & 3.93980 \\
\hline C & -2.01840 & 0.74560 & -3.35440 & $C$ & -8.17580 & 3.47250 & 3.62190 \\
\hline C & -2.00570 & 2.07820 & -3.82840 & $C$ & -6.89490 & 3.03800 & 5.71760 \\
\hline C & -0.85810 & 2.84060 & -3.75170 & $\mathrm{C}$ & -7.63100 & 2.54400 & 4.60380 \\
\hline C & 0.33420 & 2.34370 & -3.15780 & $\mathrm{C}$ & -7.91400 & 1.15460 & 4.56520 \\
\hline C & -0.79290 & -1.22770 & -2.57560 & $\mathrm{C}$ & -7.40650 & 0.28410 & 5.50390 \\
\hline C & -2.00350 & -1.96860 & -2.48850 & $\mathrm{H}$ & -8.57150 & 0.74330 & 3.81140 \\
\hline C & -3.18960 & -1.34490 & -2.95390 & $\mathrm{C}$ & -6.34130 & 2.14000 & 6.68220 \\
\hline C & -3.19950 & -0.03550 & -3.38740 & $\mathrm{C}$ & -6.59590 & 0.75440 & 6.56490 \\
\hline C & 1.63180 & 0.30840 & -2.65540 & $C$ & -6.10670 & -0.13420 & 7.55120 \\
\hline C & 1.66730 & -1.10780 & -2.49630 & $\mathrm{C}$ & -5.33800 & 0.33040 & 8.59710 \\
\hline C & 0.46750 & -1.86940 & -2.38710 & $\mathrm{C}$ & -5.01110 & 1.70460 & 8.72500 \\
\hline C & 2.83780 & 1.05880 & -2.71130 & $\mathrm{C}$ & -5.58240 & 2.61650 & 7.79310 \\
\hline C & 4.04840 & 0.33230 & -2.86800 & $\mathrm{H}$ & -5.04090 & -0.39130 & 9.34620 \\
\hline C & 4.09620 & -1.03740 & -2.73040 & $C$ & -7.32560 & 5.35750 & 5.01400 \\
\hline C & 2.91510 & -1.77240 & -2.48600 & $\mathrm{C}$ & -6.69710 & 4.44190 & 5.89170 \\
\hline C & 2.96100 & -3.17250 & -2.30180 & $C$ & -6.34060 & 7.21440 & 6.25010 \\
\hline C & 1.79910 & -3.89320 & -2.14560 & $\mathrm{C}$ & -7.11970 & 6.74550 & 5.21610 \\
\hline C & 0.52570 & -3.27050 & -2.19420 & $\mathrm{H}$ & -7.55140 & 7.48570 & 4.55580 \\
\hline C & -4.46270 & 0.55570 & -3.89760 & C & -5.75350 & 6.31290 & 7.16890 \\
\hline$N$ & -4.39060 & 1.87060 & -4.38080 & C & -5.93770 & 4.92090 & 7.00020 \\
\hline C & -3.22750 & 2.66000 & -4.44530 & C & -5.40970 & 4.02260 & 7.97160 \\
\hline C & 5.40390 & -1.73590 & -2.84570 & $\mathrm{C}$ & -4.77590 & 4.52310 & 9.13960 \\
\hline$N$ & 5.40600 & -3.10770 & -2.54200 & $\mathrm{C}$ & -4.50920 & 5.91560 & 9.21190 \\
\hline C & 4.26500 & -3.88450 & -2.29820 & $\mathrm{C}$ & -4.98630 & 6.78840 & 8.25730 \\
\hline $\mathrm{O}$ & -5.52560 & -0.05130 & -3.91300 & $\mathrm{H}$ & -3.90440 & 6.33200 & 10.00580 \\
\hline $\mathrm{O}$ & -3.23830 & 3.76420 & -4.96950 & C & -4.70710 & 8.24150 & 8.39150 \\
\hline $\mathrm{O}$ & 4.35780 & -5.08950 & -2.11030 & $\mathrm{~N}$ & -5.33050 & 9.09310 & 7.46750 \\
\hline $\mathrm{O}$ & 6.43320 & -1.16050 & -3.16480 & $\mathrm{C}$ & -6.14220 & 8.68020 & 6.39920 \\
\hline C & -5.63520 & 2.43290 & -4.91120 & $\mathrm{C}$ & -6.45000 & -1.57990 & 7.49820 \\
\hline C & 6.69440 & -3.80790 & -2.56590 & $\mathrm{~N}$ & -7.21830 & -1.99790 & 6.39770 \\
\hline C & -1.96520 & -3.35720 & -2.04410 & $\mathrm{C}$ & -7.73640 & -1.15960 & 5.40160 \\
\hline C & -0.70950 & -4.02300 & -2.07620 & $\mathrm{O}$ & -3.98660 & 8.70140 & 9.26510 \\
\hline C & -0.66950 & -5.44710 & -2.05540 & $\mathrm{O}$ & -6.65430 & 9.48890 & 5.63740 \\
\hline C & -1.81880 & -6.17540 & -2.04540 & $\mathrm{O}$ & -6.08740 & -2.36990 & 8.35750 \\
\hline C & -3.07650 & -5.54160 & -1.87880 & $\mathrm{O}$ & -8.42780 & -1.62180 & 4.50390 \\
\hline C & -3.15050 & -4.12960 & -1.70160 & $\mathrm{C}$ & -5.08370 & 10.52750 & 7.64410 \\
\hline C & -4.25390 & -6.33280 & -1.88980 & C & -7.55240 & -3.42000 & 6.28130 \\
\hline C & -5.47710 & -5.75490 & -1.74400 & $\mathrm{H}$ & -5.70710 & 11.06310 & 6.93270 \\
\hline C & -5.58600 & -4.38200 & -1.38810 & $\mathrm{H}$ & -5.32620 & 10.81480 & 8.66850 \\
\hline C & -4.41060 & -3.60400 & -1.20000 & $\mathrm{H}$ & -4.02870 & 10.75050 & 7.46680 \\
\hline C & -6.89250 & -3.79780 & -1.17210 & $\mathrm{H}$ & -7.27060 & -3.77940 & 5.29020 \\
\hline
\end{tabular}




\begin{tabular}{|c|c|c|c|c|c|c|c|}
\hline C & -6.98860 & -2.51180 & -0.59130 & $\mathrm{H}$ & -7.01340 & -3.95480 & 7.05900 \\
\hline C & -5.81020 & -1.82790 & -0.15940 & $\mathrm{H}$ & -8.62930 & -3.56300 & 6.40070 \\
\hline C & -4.52530 & -2.37970 & -0.41580 & C & -4.36330 & 3.59030 & 10.17200 \\
\hline C & -8.08070 & -4.45560 & -1.57610 & C & -4.24140 & 2.21840 & 9.84590 \\
\hline C & -9.31090 & -3.85020 & -1.45490 & C & -4.10420 & 4.00340 & 11.53410 \\
\hline C & -9.42190 & -2.56170 & -0.88340 & C & -3.39890 & 3.13880 & 12.40790 \\
\hline C & -8.26280 & -1.90590 & -0.40700 & C & -4.41920 & 5.58780 & 13.36760 \\
\hline C & -10.67660 & -1.91350 & -0.79710 & C & -4.65100 & 5.20560 & 12.07180 \\
\hline C & -10.77160 & -0.65110 & -0.25510 & C & -5.03160 & 6.84720 & 13.86810 \\
\hline C & -9.63690 & 0.01330 & 0.27190 & $\mathrm{H}$ & -5.31960 & 5.82380 & 11.48900 \\
\hline C & -8.38280 & -0.63480 & 0.22130 & C & -3.60720 & 4.79160 & 14.22300 \\
\hline C & -10.52280 & -4.55330 & -1.94520 & C & -3.10240 & 3.54940 & 13.74680 \\
\hline $\mathrm{N}$ & -11.74090 & -3.86910 & -1.83300 & C & -2.31100 & 2.73700 & 14.61190 \\
\hline C & -11.90670 & -2.58080 & -1.29670 & C & -2.05550 & 3.20360 & 15.91090 \\
\hline $\mathrm{O}$ & -13.00680 & -2.04680 & -1.24980 & C & -2.55900 & 4.41910 & 16.36830 \\
\hline $\mathrm{O}$ & -10.49090 & -5.67550 & -2.43150 & C & -3.33630 & 5.21730 & 15.53650 \\
\hline C & -12.92300 & -4.57920 & -2.33030 & $\mathrm{H}$ & -1.45350 & 2.61330 & 16.59070 \\
\hline $\mathrm{H}$ & -0.90160 & 3.82740 & -4.18760 & $\mathrm{H}$ & -2.35780 & 4.76350 & 17.37680 \\
\hline $\mathrm{H}$ & -4.11900 & -1.89660 & -3.00820 & C & -3.88120 & 6.49970 & 16.05070 \\
\hline $\mathrm{H}$ & 4.97270 & 0.82550 & -3.12600 & C & -2.95920 & 1.86140 & 11.93940 \\
\hline $\mathrm{H}$ & 1.90960 & -4.95710 & -1.98740 & C & -3.36590 & 1.39670 & 10.65970 \\
\hline $\mathrm{H}$ & -5.48270 & 3.49770 & -5.06650 & C & -2.80280 & 0.17560 & 10.18490 \\
\hline $\mathrm{H}$ & -5.89620 & 1.95270 & -5.85820 & C & -1.96580 & -0.58770 & 10.96150 \\
\hline $\mathrm{H}$ & -6.44110 & 2.24960 & -4.20030 & $\mathrm{H}$ & -2.97480 & -0.16050 & 9.17240 \\
\hline $\mathrm{H}$ & 7.48040 & -3.08050 & -2.37630 & C & -1.40010 & -1.84230 & 10.38980 \\
\hline $\mathrm{H}$ & 6.68100 & -4.58650 & -1.80500 & C & -1.64120 & -0.18670 & 12.28870 \\
\hline $\mathrm{H}$ & 6.86340 & -4.27350 & -3.54190 & C & -2.13250 & 1.05300 & 12.78250 \\
\hline $\mathrm{H}$ & 0.27730 & -5.96410 & -2.12920 & C & -1.79430 & 1.46430 & 14.10880 \\
\hline $\mathrm{H}$ & -1.78810 & -7.25720 & -2.13610 & C & -0.98540 & 0.61800 & 14.88310 \\
\hline $\mathrm{H}$ & -4.16120 & -7.40270 & -2.05170 & C & -0.49880 & -0.58800 & 14.38340 \\
\hline $\mathrm{H}$ & -6.36630 & -6.36690 & -1.82460 & C & -0.81780 & -0.99650 & 13.09330 \\
\hline $\mathrm{H}$ & -8.06090 & -5.43240 & -2.04130 & $\mathrm{H}$ & -0.71860 & 0.89920 & 15.89480 \\
\hline $\mathrm{H}$ & -11.75920 & -0.21080 & -0.20830 & $\mathrm{H}$ & 0.13260 & -1.22920 & 14.98870 \\
\hline $\mathrm{H}$ & -12.79200 & -4.81950 & -3.38760 & C & -0.28130 & -2.27790 & 12.57490 \\
\hline $\mathrm{H}$ & -13.04690 & -5.51650 & -1.78340 & $\mathrm{~N}$ & -4.68400 & 7.24000 & 15.16660 \\
\hline $\mathrm{H}$ & -13.78540 & -3.93380 & -2.18560 & $\mathrm{~N}$ & -0.61390 & -2.61800 & 11.25620 \\
\hline C & -5.94380 & -0.59410 & 0.55180 & $\mathrm{O}$ & -3.65460 & 6.89690 & 17.18570 \\
\hline C & -7.22440 & 0.00630 & 0.75930 & $\mathrm{O}$ & -5.79440 & 7.52990 & 13.19800 \\
\hline C & -7.34470 & 1.22070 & 1.49200 & $\mathrm{O}$ & 0.42850 & -3.01880 & 13.24220 \\
\hline C & -6.14790 & 1.86230 & 1.90130 & $\mathrm{O}$ & -1.60750 & -2.18940 & 9.23530 \\
\hline C & -4.91130 & 1.27920 & 1.73250 & C & -5.25450 & 8.50640 & 15.63570 \\
\hline C & -4.78710 & 0.04240 & 1.05820 & C & -0.06070 & -3.88060 & 10.75800 \\
\hline C & -3.40350 & -1.74610 & 0.17810 & $\mathrm{H}$ & -4.74440 & 8.78450 & 16.55420 \\
\hline C & -3.52170 & -0.56490 & 0.87880 & $\mathrm{H}$ & -5.12050 & 9.26560 & 14.86460 \\
\hline C & -2.30190 & 0.09370 & 1.41060 & $\mathrm{H}$ & -6.32570 & 8.39380 & 15.82390 \\
\hline $\mathrm{N}$ & -2.48170 & 1.31260 & 2.08240 & $\mathrm{H}$ & -0.32290 & -4.68750 & 11.44430 \\
\hline C & -3.71080 & 1.96720 & 2.27600 & $\mathrm{H}$ & -0.47280 & -4.05850 & 9.76820 \\
\hline $\mathrm{O}$ & -3.77730 & 3.03600 & 2.86960 & $\mathrm{H}$ & 1.02920 & -3.81750 & 10.70930 \\
\hline $\mathrm{O}$ & -1.17730 & -0.36890 & 1.27230 & C & 2.76460 & 2.51080 & -2.65570 \\
\hline C & -1.26620 & 1.93710 & 2.61160 & C & 1.51700 & 3.15420 & -2.88310 \\
\hline $\mathrm{H}$ & -6.17270 & 2.83180 & 2.37360 & C & 2.66290 & 5.35730 & -2.86890 \\
\hline $\mathrm{H}$ & -1.55840 & 2.84330 & 3.13610 & C & 1.45800 & 4.60540 & -2.83050 \\
\hline $\mathrm{H}$ & -0.76080 & 1.24430 & 3.28780 & C & 0.24100 & 5.33970 & -2.69800 \\
\hline $\mathrm{H}$ & -0.58170 & 2.17330 & 1.79350 & C & 0.19490 & 6.70280 & -2.85170 \\
\hline $\mathrm{H}$ & -2.41850 & -2.18240 & 0.10030 & $\mathrm{H}$ & -0.68710 & 4.84540 & -2.44940 \\
\hline C & -8.65580 & 1.84330 & 1.64570 & C & -1.11860 & 7.39570 & -2.75750 \\
\hline C & -9.73140 & 1.32490 & 0.87730 & C & 1.38220 & 7.44440 & -3.10270 \\
\hline C & -10.87770 & 2.13130 & 0.63100 & C & 2.63460 & 6.77720 & -3.04990 \\
\hline C & -10.92120 & 3.41710 & 1.07760 & C & 3.83690 & 7.53840 & -3.15950 \\
\hline $\mathrm{H}$ & -11.66370 & 1.76490 & -0.01670 & C & 3.73210 & 8.91160 & -3.42550 \\
\hline $\mathrm{H}$ & -11.73190 & 4.07760 & 0.78430 & C & 2.49700 & 9.54900 & -3.51760 \\
\hline C & -8.89240 & 3.04680 & 2.42780 & C & 1.32100 & 8.83110 & -3.33080 \\
\hline C & -9.93280 & 3.90630 & 1.97120 & $\mathrm{H}$ & 4.62650 & 9.51020 & -3.54200 \\
\hline C & -9.98880 & 5.25670 & 2.40530 & $\mathrm{H}$ & 2.43030 & 10.61460 & -3.70820 \\
\hline C & -9.06790 & 5.74050 & 3.28420 & C & 0.01640 & 9.53440 & -3.34950 \\
\hline $\mathrm{H}$ & -10.76370 & 5.90270 & 2.00250 & C & 3.91370 & 4.70800 & -2.63920 \\
\hline
\end{tabular}



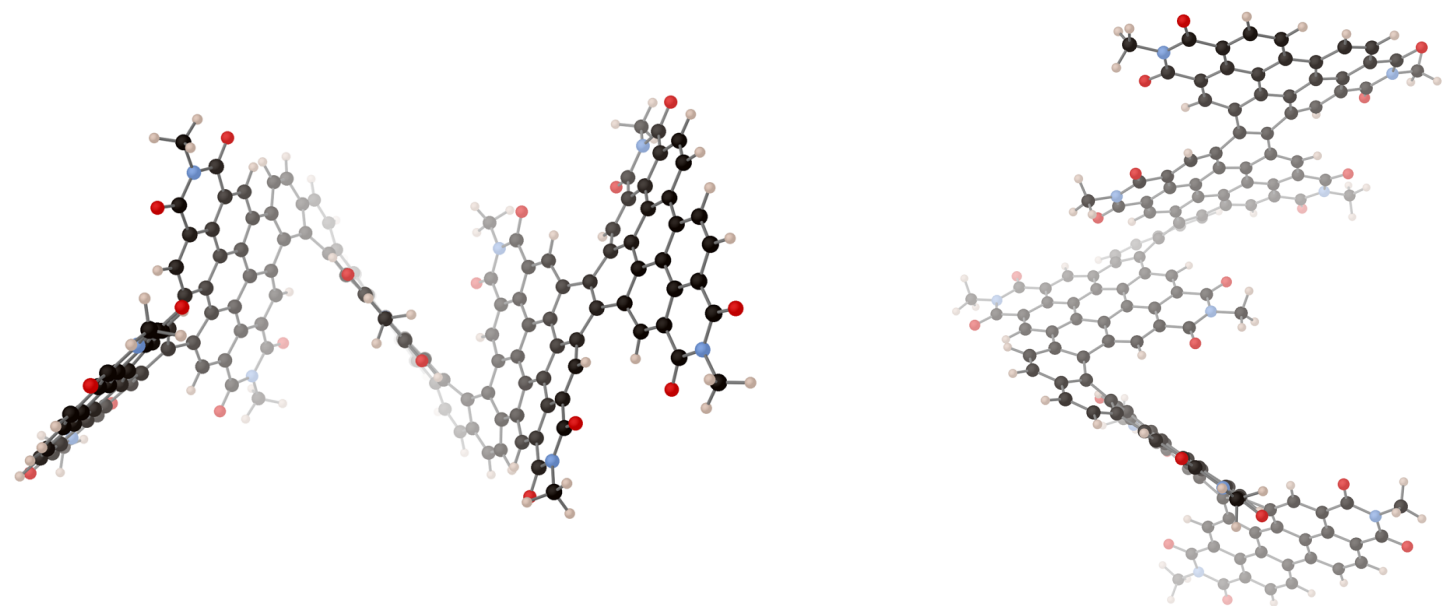

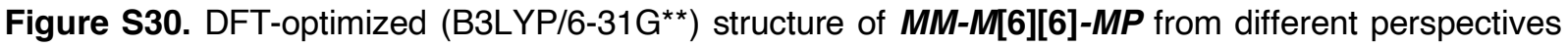
(total energy $=-7958.7792$ hartrees).

Cartesian Coordinates of $\mathbf{M M - M}$ 6][6]-MP (from left to right: atom; x, y, z in $\AA$ )

\begin{tabular}{|c|c|c|c|c|c|c|c|c|c|c|c|}
\hline C & 1.01620 & 1.77670 & -2.39370 & $\mathrm{H}$ & -11.19950 & 5.79410 & 2.54690 & C & 1.14080 & 8.75450 & -7.41260 \\
\hline C & -0.16680 & 0.98290 & -2.28050 & $\mathrm{H}$ & -9.83500 & 6.53100 & 4.45020 & C & 4.62340 & 5.29520 & -3.53670 \\
\hline C & -1.39800 & 1.50710 & -2.73150 & C & -8.84980 & 4.60990 & 4.71300 & C & 4.66890 & 4.02390 & -2.90360 \\
\hline C & -1.45460 & 2.84350 & -3.18870 & C & -8.72970 & 3.28590 & 4.22130 & C & 5.96170 & 3.50580 & -2.59440 \\
\hline C & -0.30570 & 3.60180 & -3.28850 & C & -7.84480 & 2.60310 & 6.44210 & C & 7.08950 & 4.28530 & -2.64070 \\
\hline C & 0.97770 & 3.05560 & -3.01390 & C & -8.32310 & 2.24160 & 5.15180 & $\mathrm{H}$ & 6.10000 & 2.47480 & -2.30880 \\
\hline C & -0.13230 & -0.32700 & -1.71460 & C & -8.54090 & 0.86340 & 4.88660 & C & 8.38850 & 3.69450 & -2.20920 \\
\hline C & -1.30980 & -1.12270 & -1.64050 & C & -8.24700 & -0.10910 & 5.81640 & C & 7.01580 & 5.63760 & -3.07460 \\
\hline C & -2.49720 & -0.59110 & -2.20600 & $\mathrm{H}$ & -8.98900 & 0.53760 & 3.95910 & C & 5.78710 & 6.12740 & -3.59420 \\
\hline C & -2.55870 & 0.69690 & -2.69170 & C & -7.52530 & 1.60030 & 7.40680 & C & 5.74330 & 7.42990 & -4.17630 \\
\hline C & 2.22350 & 1.31810 & -1.78780 & C & -7.73480 & 0.23830 & 7.08930 & C & 6.89100 & 8.23370 & -4.09180 \\
\hline C & 2.23580 & 0.04750 & -1.14230 & C & -7.46530 & -0.75390 & 8.06000 & C & 8.07960 & 7.75640 & -3.54090 \\
\hline C & 1.10070 & -0.81050 & -1.18420 & C & -7.05450 & -0.40090 & 9.32940 & C & 8.16090 & 6.45270 & -3.06290 \\
\hline C & 3.37930 & 2.14560 & -1.77120 & C & -6.88680 & 0.95630 & 9.70770 & $\mathrm{H}$ & 6.87480 & 9.24240 & -4.48390 \\
\hline C & 4.40840 & 1.78660 & -0.85610 & C & -7.05130 & 1.95060 & 8.70720 & $\mathrm{H}$ & 8.96870 & 8.37640 & -3.50430 \\
\hline C & 4.44440 & 0.54820 & -0.25130 & $\mathrm{H}$ & -6.83930 & -1.20490 & 10.01860 & C & 9.45930 & 5.91950 & -2.57700 \\
\hline C & 3.39050 & -0.37300 & -0.44400 & C & -8.20550 & 4.98810 & 5.95520 & $\mathrm{~N}$ & 0.17100 & 7.74820 & -7.56060 \\
\hline C & 3.44270 & -1.66990 & 0.11830 & C & -7.71550 & 3.97650 & 6.81290 & $\mathrm{~N}$ & 9.48610 & 4.57130 & -2.19130 \\
\hline C & 2.38900 & -2.54210 & -0.04820 & C & -7.44230 & 6.68770 & 7.52560 & $\mathrm{O}$ & 1.06220 & 9.81130 & -8.02430 \\
\hline C & 1.19850 & -2.13340 & -0.69880 & C & -8.04510 & 6.34330 & 6.33520 & $\mathrm{O}$ & -0.71330 & 5.69640 & -7.11420 \\
\hline C & -3.86910 & 1.23520 & -3.15130 & $\mathrm{H}$ & -8.36420 & 7.15150 & 5.69150 & $\mathrm{O}$ & 8.50020 & 2.52280 & -1.87770 \\
\hline $\mathrm{N}$ & -3.87610 & 2.58370 & -3.54840 & C & -6.99440 & 5.68530 & 8.41920 & $\mathrm{O}$ & 10.47700 & 6.59690 & -2.52060 \\
\hline C & -2.76150 & 3.43880 & -3.57830 & C & -7.14580 & 4.32250 & 8.07430 & C & -0.94980 & 7.98350 & -8.47740 \\
\hline C & 5.60220 & 0.19670 & 0.61890 & C & -6.77070 & 3.31730 & 9.01150 & C & 10.78160 & 4.05540 & -1.73580 \\
\hline $\mathrm{N}$ & 5.61510 & -1.11570 & 1.12890 & C & -6.14250 & 3.66660 & 10.23790 & $\mathrm{H}$ & -0.82840 & 8.97820 & -8.89880 \\
\hline C & 4.62300 & -2.08480 & 0.92390 & C & -6.01450 & 5.04670 & 10.54760 & $\mathrm{H}$ & -0.94800 & 7.22880 & -9.26660 \\
\hline O & -4.89150 & 0.56180 & -3.15520 & C & -6.43250 & 6.02660 & 9.67110 & $\mathrm{H}$ & -1.89360 & 7.90720 & -7.93360 \\
\hline $\mathrm{O}$ & -2.89000 & 4.60520 & -3.92140 & $\mathrm{H}$ & -5.64140 & 5.37650 & 11.50700 & $\mathrm{H}$ & 11.51430 & 4.12280 & -2.54310 \\
\hline O & 4.73900 & -3.20080 & 1.41250 & C & -6.31670 & 7.45580 & 10.06810 & $\mathrm{H}$ & 11.14090 & 4.65410 & -0.89680 \\
\hline $\mathrm{O}$ & 6.49850 & 0.98900 & 0.86990 & $\mathrm{~N}$ & -6.74220 & 8.40900 & 9.13030 & $\mathrm{H}$ & 10.63740 & 3.02040 & -1.43640 \\
\hline C & -5.14640 & 3.17870 & -3.97750 & C & -7.29580 & 8.12670 & 7.86980 & & & & \\
\hline C & 6.75210 & -1.52810 & 1.95930 & C & -7.65180 & -2.19450 & 7.73570 & & & & \\
\hline C & -1.22900 & -2.46900 & -1.08060 & $\mathrm{~N}$ & -8.17450 & -2.48140 & 6.46110 & & & & \\
\hline C & 0.05410 & -3.01000 & -0.81150 & C & -8.50420 & -1.53520 & 5.48070 & & & & \\
\hline C & 0.22010 & -4.41950 & -0.69280 & $\mathrm{O}$ & -5.87400 & 7.81170 & 11.15080 & & & & \\
\hline C & -0.83680 & -5.25830 & -0.88270 & $\mathrm{O}$ & -7.64890 & 9.01950 & 7.11170 & & & & \\
\hline C & -2.15600 & -4.74200 & -0.98180 & $\mathrm{O}$ & -7.37750 & -3.08420 & 8.52780 & & & & \\
\hline C & -2.37710 & -3.33760 & -0.87790 & $\mathrm{O}$ & -8.96190 & -1.89500 & 4.40410 & & & & \\
\hline C & -3.25680 & -5.61930 & -1.16940 & C & -6.59590 & 9.81170 & 9.53420 & & & & \\
\hline C & -4.52800 & -5.13270 & -1.23420 & $\mathrm{C}$ & -8.41730 & -3.88410 & 6.10800 & & & & \\
\hline C & -4.79820 & -3.77270 & -0.90990 & $\mathrm{H}$ & -6.93900 & 10.43080 & 8.70920 & & & & \\
\hline C & -3.72700 & -2.90900 & -0.56360 & $\mathrm{H}$ & -7.18960 & 10.00380 & 10.43070 & & & & \\
\hline
\end{tabular}




\begin{tabular}{|c|c|c|c|c|c|c|c|}
\hline C & -6.16230 & -3.28720 & -0.88150 & $\mathrm{H}$ & -5.54970 & 10.02120 & 9.76580 \\
\hline C & -6.43850 & -2.04420 & -0.27030 & $\mathrm{H}$ & -7.82410 & -4.15580 & 5.23220 \\
\hline C & -5.38590 & -1.29480 & 0.34340 & $\mathrm{H}$ & -8.13780 & -4.49330 & 6.96390 \\
\hline C & -4.03280 & -1.71730 & 0.22080 & $\mathrm{H}$ & -9.47220 & -4.02540 & 5.86340 \\
\hline C & -7.22320 & -4.00300 & -1.49170 & $\mathrm{C}$ & -5.82910 & 2.61650 & 11.19750 \\
\hline C & -8.50700 & -3.50180 & -1.51660 & $\mathrm{C}$ & -6.44320 & 1.35260 & 11.03780 \\
\hline C & -8.80220 & -2.26520 & -0.89630 & $\mathrm{C}$ & -4.94150 & 2.81530 & 12.32920 \\
\hline C & -7.77250 & -1.55240 & -0.23470 & $\mathrm{C}$ & -4.98280 & 1.91500 & 13.42380 \\
\hline C & -10.11030 & -1.72510 & -0.94000 & $\mathrm{C}$ & -3.08300 & 3.99420 & 13.39520 \\
\hline C & -10.38450 & -0.51300 & -0.34230 & $\mathrm{C}$ & -3.92930 & 3.82010 & 12.32840 \\
\hline C & -9.38460 & 0.20170 & 0.36330 & $\mathrm{C}$ & -2.04780 & 5.06250 & 13.33120 \\
\hline C & -8.08270 & -0.34210 & 0.44380 & $\mathrm{H}$ & -3.77120 & 4.45190 & 11.46480 \\
\hline C & -9.58040 & -4.26110 & -2.21330 & $\mathrm{C}$ & -3.19490 & 3.17550 & 14.55350 \\
\hline $\mathrm{N}$ & -10.86350 & -3.69310 & -2.20300 & $\mathrm{C}$ & -4.14700 & 2.12000 & 14.56840 \\
\hline C & -11.20970 & -2.46170 & -1.62080 & $\mathrm{C}$ & -4.25100 & 1.28760 & 15.72180 \\
\hline $\mathrm{O}$ & -12.35270 & -2.02830 & -1.67760 & $\mathrm{C}$ & -3.39290 & 1.53490 & 16.80260 \\
\hline 0 & -9.38690 & -5.33260 & -2.77220 & $\mathrm{C}$ & -2.45730 & 2.56590 & 16.77500 \\
\hline C & -11.91390 & -4.46610 & -2.87450 & $\mathrm{C}$ & -2.35030 & 3.38870 & 15.65900 \\
\hline $\mathrm{H}$ & -0.42670 & 4.63040 & -3.59220 & $\mathrm{H}$ & -3.44600 & 0.91440 & 17.68880 \\
\hline $\mathrm{H}$ & -3.40030 & -1.18040 & -2.25400 & $\mathrm{H}$ & -1.79550 & 2.74430 & 17.61550 \\
\hline $\mathrm{H}$ & 5.21210 & 2.46380 & -0.61450 & $\mathrm{C}$ & -1.33000 & 4.46860 & 15.64530 \\
\hline $\mathrm{H}$ & 2.47820 & -3.52480 & 0.39900 & $\mathrm{C}$ & -5.89300 & 0.81540 & 13.39800 \\
\hline $\mathrm{H}$ & -5.06330 & 3.52630 & -5.00960 & $\mathrm{C}$ & -6.62530 & 0.53000 & 12.21690 \\
\hline $\mathrm{H}$ & -5.91510 & 2.41490 & -3.89160 & $\mathrm{C}$ & -7.62830 & -0.48100 & 12.28040 \\
\hline $\mathrm{H}$ & -5.38410 & 4.03750 & -3.34640 & $\mathrm{C}$ & -7.82850 & -1.23270 & 13.40990 \\
\hline $\mathrm{H}$ & 7.46010 & -0.70350 & 1.98320 & $\mathrm{H}$ & -8.29740 & -0.65540 & 11.45050 \\
\hline $\mathrm{H}$ & 6.40950 & -1.76730 & 2.96870 & $\mathrm{C}$ & -8.90670 & -2.26290 & 13.40240 \\
\hline $\mathrm{H}$ & 7.21210 & -2.42170 & 1.53320 & $\mathrm{C}$ & -7.02280 & -1.03170 & 14.56550 \\
\hline $\mathrm{H}$ & 1.21260 & -4.82740 & -0.54360 & $\mathrm{C}$ & -6.04990 & 0.00570 & 14.56670 \\
\hline $\mathrm{H}$ & -0.69120 & -6.33390 & -0.92380 & $\mathrm{C}$ & -5.25630 & 0.22310 & 15.73400 \\
\hline $\mathrm{H}$ & -3.06380 & -6.68230 & -1.28050 & $\mathrm{C}$ & -5.47360 & -0.60440 & 16.84620 \\
\hline $\mathrm{H}$ & -5.35030 & -5.80660 & -1.43980 & C & -6.43890 & -1.60840 & 16.83870 \\
\hline $\mathrm{H}$ & -7.05070 & -4.94150 & -2.00190 & C & -7.21830 & -1.82590 & 15.70910 \\
\hline $\mathrm{H}$ & -11.40400 & -0.15180 & -0.39580 & $\mathrm{H}$ & -4.88950 & -0.46490 & 17.74630 \\
\hline $\mathrm{H}$ & -11.70080 & -4.53940 & -3.94350 & $\mathrm{H}$ & -6.60470 & -2.23220 & 17.71030 \\
\hline $\mathrm{H}$ & -11.94480 & -5.47580 & -2.46190 & $\mathrm{C}$ & -8.26840 & -2.87480 & 15.73390 \\
\hline $\mathrm{H}$ & -12.85800 & -3.95210 & -2.71210 & $\mathrm{~N}$ & -1.24800 & 5.23260 & 14.46930 \\
\hline C & -5.71770 & -0.13600 & 1.10710 & $\mathrm{~N}$ & -9.03970 & -3.02730 & 14.57200 \\
\hline C & -7.06450 & 0.33020 & 1.18980 & $\mathrm{O}$ & -0.59230 & 4.68190 & 16.59810 \\
\hline C & -7.39180 & 1.44870 & 2.00300 & $\mathrm{O}$ & -1.88420 & 5.77480 & 12.35070 \\
\hline C & -6.32970 & 2.14580 & 2.63270 & $\mathrm{O}$ & -8.47800 & -3.57810 & 16.71300 \\
\hline C & -5.02400 & 1.70940 & 2.55060 & $\mathrm{O}$ & -9.63830 & -2.44230 & 12.43870 \\
\hline C & -4.69640 & 0.55840 & 1.79550 & $\mathrm{C}$ & -0.24860 & 6.30390 & 14.39750 \\
\hline C & -3.04470 & -1.01380 & 0.95830 & C & -10.08310 & -4.05760 & 14.61080 \\
\hline C & -3.36120 & 0.09200 & 1.71850 & $\mathrm{H}$ & 0.29200 & 6.31440 & 15.34080 \\
\hline C & -2.28120 & 0.80300 & 2.45300 & $\mathrm{H}$ & 0.43310 & 6.11860 & 13.56490 \\
\hline $\mathrm{N}$ & -2.65750 & 1.95240 & 3.16570 & $\mathrm{H}$ & -0.74480 & 7.26160 & 14.22710 \\
\hline C & -3.96190 & 2.47140 & 3.26240 & $\mathrm{H}$ & -9.67680 & -4.95940 & 15.06770 \\
\hline $\mathrm{O}$ & -4.19680 & 3.48550 & 3.90650 & $\mathrm{H}$ & -10.93100 & -3.71770 & 15.21220 \\
\hline $\mathrm{O}$ & -1.11120 & 0.44150 & 2.44140 & $\mathrm{H}$ & -10.40790 & -4.24240 & 13.59020 \\
\hline C & -1.57540 & 2.65050 & 3.86610 & $\mathrm{C}$ & 3.42780 & 3.31080 & -2.64530 \\
\hline $\mathrm{H}$ & -6.51590 & 3.05060 & 3.19190 & $\mathrm{C}$ & 2.23550 & 3.74370 & -3.28790 \\
\hline $\mathrm{H}$ & -2.00650 & 3.51960 & 4.35680 & $\mathrm{C}$ & 3.43310 & 5.70950 & -4.20870 \\
\hline $\mathrm{H}$ & -1.11600 & 1.98400 & 4.59930 & $\mathrm{C}$ & 2.29720 & 4.86000 & -4.21520 \\
\hline $\mathrm{H}$ & -0.80690 & 2.95360 & 3.15220 & $\mathrm{C}$ & 1.29180 & 5.12160 & -5.19470 \\
\hline $\mathrm{H}$ & -2.01470 & -1.34140 & 0.96420 & $\mathrm{C}$ & 1.28250 & 6.27650 & -5.93430 \\
\hline C & -8.76480 & 1.94040 & 2.01920 & $\mathrm{H}$ & 0.50480 & 4.40900 & -5.39260 \\
\hline C & -9.67190 & 1.44530 & 1.05030 & $\mathrm{C}$ & 0.17080 & 6.51320 & -6.89730 \\
\hline C & -10.82750 & 2.20940 & 0.71460 & $\mathrm{C}$ & 2.31560 & 7.24200 & -5.77870 \\
\hline C & -11.03540 & 3.43110 & 1.28700 & $\mathrm{C}$ & 3.42940 & 6.93360 & -4.95030 \\
\hline $\mathrm{H}$ & -11.47970 & 1.87020 & -0.07990 & C & 4.53140 & 7.83760 & -4.89350 \\
\hline $\mathrm{H}$ & -11.84030 & 4.07390 & 0.94240 & $\mathrm{C}$ & 4.42740 & 9.05110 & -5.58820 \\
\hline C & -9.21620 & 3.00990 & 2.88490 & $\mathrm{C}$ & 3.31690 & 9.35910 & -6.37110 \\
\hline C & -10.23600 & 3.86300 & 2.38100 & $\mathrm{C}$ & 2.27040 & 8.45140 & -6.49490 \\
\hline C & -10.44560 & 5.13850 & 2.97280 & $\mathrm{H}$ & 5.23790 & 9.76700 & -5.54920 \\
\hline C & -9.69890 & 5.53770 & 4.04200 & $\mathrm{H}$ & 3.26180 & 10.29170 & -6.92190 \\
\hline
\end{tabular}



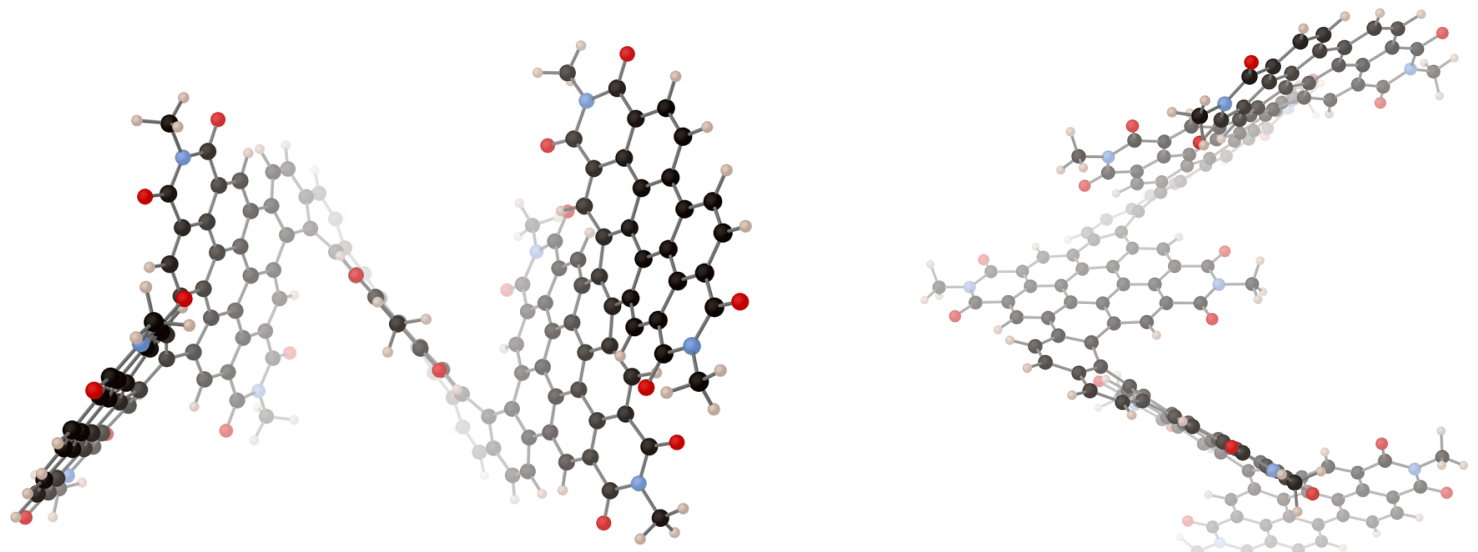

Figure S31. DFT-optimized (B3LYP/6-31G**) structure of $M$ M-M[6][6]-PM from different perspectives (total energy $=-7958.7833$ hartrees).

Cartesian Coordinates of $\mathbf{M M - M}$ 6][6]-PM (from left to right: atom; x, y, z in $\AA$ )

\begin{tabular}{|c|c|c|c|c|c|c|c|c|c|c|c|}
\hline C & 0.66150 & 2.07630 & -1.81090 & $\mathrm{H}$ & -10.49620 & 6.33620 & 4.41110 & C & 3.92090 & 4.84800 & -1.40570 \\
\hline C & -0.42830 & 1.15280 & -1.86390 & C & -9.35640 & 4.50430 & 4.68840 & C & 5.06340 & 4.55260 & -0.60320 \\
\hline C & -1.63460 & 1.54160 & -2.49080 & $\mathrm{C}$ & -9.12070 & 3.19500 & 4.19560 & C & 6.14450 & 5.39540 & -0.54660 \\
\hline C & -1.70540 & 2.79400 & -3.14110 & $\mathrm{C}$ & -8.16960 & 2.59630 & 6.41750 & $\mathrm{H}$ & 5.10440 & 3.66030 & 0.00430 \\
\hline C & -0.64810 & 3.67660 & -3.07960 & $\mathrm{C}$ & -8.62870 & 2.19270 & 5.13330 & C & 7.32850 & 5.00590 & 0.26330 \\
\hline C & 0.51700 & 3.40110 & -2.31520 & $\mathrm{C}$ & -8.72270 & 0.80130 & 4.87470 & C & 6.13960 & 6.62260 & -1.26820 \\
\hline C & -0.30080 & -0.17750 & -1.35380 & $\mathrm{C}$ & -8.32920 & -0.13860 & 5.79790 & C & 4.95090 & 7.03260 & -1.93170 \\
\hline C & -1.41990 & -1.05330 & -1.33020 & $\mathrm{H}$ & -9.14240 & 0.43100 & 3.95350 & C & 4.89630 & 8.31800 & -2.54790 \\
\hline C & -2.60480 & -0.61910 & -1.97970 & $\mathrm{C}$ & -7.74420 & 1.62760 & 7.37690 & C & 6.06300 & 9.09920 & -2.55710 \\
\hline C & -2.71080 & 0.62550 & -2.55820 & C & -7.82720 & 0.25430 & 7.05970 & C & 7.23210 & 8.67450 & -1.92880 \\
\hline C & 1.94320 & 1.61140 & -1.38570 & $\mathrm{C}$ & -7.42010 & -0.71020 & 8.00800 & C & 7.27370 & 7.45470 & -1.26100 \\
\hline C & 2.10810 & 0.24030 & -1.02920 & $\mathrm{C}$ & -6.99560 & -0.32710 & 9.26170 & $\mathrm{H}$ & 6.06000 & 10.07350 & -3.02800 \\
\hline C & 0.98980 & -0.64160 & -0.95190 & $\mathrm{C}$ & -6.94950 & 1.03770 & 9.64430 & $\mathrm{H}$ & 8.12030 & 9.29680 & -1.92330 \\
\hline C & 3.06990 & 2.47380 & -1.45520 & $\mathrm{C}$ & -7.26050 & 2.01570 & 8.66180 & C & 8.49910 & 7.06520 & -0.51730 \\
\hline C & 4.35810 & 1.87760 & -1.40360 & $\mathrm{H}$ & -6.66080 & -1.11060 & 9.92510 & $\mathrm{~N}$ & -1.39740 & 9.56740 & -3.67990 \\
\hline C & 4.53060 & 0.55430 & -1.05970 & $\mathrm{C}$ & -8.75530 & 4.93550 & 5.93550 & $\mathrm{~N}$ & 8.43390 & 5.86540 & 0.21150 \\
\hline C & 3.40960 & -0.27190 & -0.81870 & C & -8.16480 & 3.97420 & 6.78880 & $\mathrm{O}$ & -0.43860 & 11.46930 & -4.53540 \\
\hline C & 3.57840 & -1.62810 & -0.45660 & $\mathrm{C}$ & -8.17120 & 6.69110 & 7.52090 & $\mathrm{O}$ & -2.35370 & 7.69370 & -2.80660 \\
\hline C & 2.48960 & -2.45890 & -0.32860 & $\mathrm{C}$ & -8.73660 & 6.29560 & 6.32830 & $\mathrm{O}$ & 7.36670 & 3.99020 & 0.94350 \\
\hline C & 1.17860 & -2.00430 & -0.61270 & $\mathrm{H}$ & -9.14840 & 7.07140 & 5.69730 & $\mathrm{O}$ & 9.50930 & 7.75590 & -0.51790 \\
\hline C & -3.95700 & 0.97630 & -3.29080 & C & -7.61080 & 5.73470 & 8.40180 & C & -2.74550 & 10.10240 & -3.89660 \\
\hline$N$ & -3.96370 & 2.22250 & -3.93860 & $\mathrm{C}$ & -7.61870 & 4.36710 & 8.04660 & C & 9.60360 & 5.45440 & 0.99470 \\
\hline C & -2.91150 & 3.15230 & -3.93380 & C & -7.11810 & 3.40170 & 8.96800 & $\mathrm{H}$ & -2.64360 & 11.08560 & -4.34950 \\
\hline C & 5.90860 & -0.00080 & -0.97300 & $\mathrm{C}$ & -6.50990 & 3.80730 & 10.18760 & $\mathrm{H}$ & -3.30710 & 9.43240 & -4.55090 \\
\hline$N$ & 6.01460 & -1.33350 & -0.53510 & $\mathrm{C}$ & -6.53980 & 5.18770 & 10.51340 & $\mathrm{H}$ & -3.27390 & 10.17100 & -2.94330 \\
\hline C & 4.93780 & -2.18360 & -0.25050 & $\mathrm{C}$ & -7.07720 & 6.12200 & 9.65310 & $\mathrm{H}$ & 10.35040 & 6.24030 & 0.91460 \\
\hline $\mathrm{O}$ & -4.92210 & 0.22450 & -3.34140 & $\mathrm{H}$ & -6.19270 & 5.54680 & 11.47180 & $\mathrm{H}$ & 9.31110 & 5.30160 & 2.03510 \\
\hline $\mathrm{O}$ & -3.01250 & 4.19770 & -4.55930 & C & -7.11330 & 7.54640 & 10.06640 & $\mathrm{H}$ & 9.99730 & 4.51140 & 0.60820 \\
\hline $\mathrm{O}$ & 5.13880 & -3.33310 & 0.11860 & $\mathrm{~N}$ & -7.64570 & 8.45840 & 9.14110 & & & & \\
\hline $\mathrm{O}$ & 6.89890 & 0.65890 & -1.25190 & C & -8.17870 & 8.13120 & 7.88240 & & & & \\
\hline C & -5.15610 & 2.61110 & -4.69860 & $\mathrm{C}$ & -7.46110 & -2.15650 & 7.67440 & & & & \\
\hline C & 7.35170 & -1.91420 & -0.37780 & $\mathrm{~N}$ & -7.99300 & -2.49100 & 6.41510 & & & & \\
\hline C & -1.27160 & -2.40070 & -0.78960 & $\mathrm{C}$ & -8.44800 & -1.57530 & 5.45580 & & & & \\
\hline C & 0.04180 & -2.90150 & -0.59380 & $\mathrm{O}$ & -6.70230 & 7.93860 & 11.14940 & & & & \\
\hline C & 0.24840 & -4.30630 & -0.45400 & $\mathrm{O}$ & -8.63720 & 8.98940 & 7.14040 & & & & \\
\hline C & -0.79470 & -5.17380 & -0.57740 & $\mathrm{O}$ & -7.06640 & -3.01640 & 8.44770 & & & & \\
\hline C & -2.12950 & -4.69870 & -0.65840 & $\mathrm{O}$ & -8.89780 & -1.97250 & 4.38920 & & & & \\
\hline C & -2.39090 & -3.30150 & -0.57610 & C & -7.64890 & 9.86410 & 9.55830 & & & & \\
\hline C & -3.19550 & -5.61930 & -0.84210 & C & -8.09530 & -3.90800 & 6.05260 & & & & \\
\hline C & -4.47780 & -5.17110 & -0.95070 & $\mathrm{H}$ & -8.06920 & 10.45080 & 8.74530 & & & & \\
\hline C & -4.79260 & -3.80910 & -0.67500 & $\mathrm{H}$ & -8.24740 & 9.97980 & 10.46460 & & & & \\
\hline C & -3.76130 & -2.90480 & -0.31550 & $\mathrm{H}$ & -6.62880 & 10.18470 & 9.77900 & & & & \\
\hline C & -6.16710 & -3.35730 & -0.72610 & $\mathrm{H}$ & -7.51400 & -4.10290 & 5.14870 & & & & \\
\hline
\end{tabular}




\begin{tabular}{|c|c|c|c|c|c|c|c|}
\hline C & -6.50790 & -2.11070 & -0.15540 & $\mathrm{H}$ & -7.71390 & -4.49300 & 6.88590 \\
\hline C & -5.50340 & -1.31370 & 0.47500 & $\mathrm{H}$ & -9.13780 & -4.16240 & 5.84950 \\
\hline C & -4.13480 & -1.69160 & 0.40260 & C & -6.03470 & 2.79420 & 11.11870 \\
\hline C & -7.17450 & -4.11300 & -1.37330 & C & -6.49530 & 1.46600 & 10.95910 \\
\hline C & -8.46800 & -3.64950 & -1.46320 & C & -5.12440 & 3.08040 & 12.21120 \\
\hline C & -8.83340 & -2.42040 & -0.86600 & C & -4.98960 & 2.15380 & 13.27750 \\
\hline C & -7.85800 & -1.66300 & -0.17610 & C & -3.35840 & 4.45060 & 13.20580 \\
\hline C & -10.16060 & -1.93620 & -0.95600 & C & -4.24680 & 4.20410 & 12.18850 \\
\hline C & -10.50260 & -0.73630 & -0.37010 & C & -2.45390 & 5.62620 & 13.10890 \\
\hline C & -9.55710 & 0.02640 & 0.36080 & $\mathrm{H}$ & -4.21350 & 4.86750 & 11.33610 \\
\hline C & -8.23510 & -0.45820 & 0.47840 & C & -3.29830 & 3.59370 & 14.34080 \\
\hline C & -9.47720 & -4.44910 & -2.19940 & C & -4.11670 & 2.43130 & 14.37820 \\
\hline $\mathrm{N}$ & -10.77910 & -3.92850 & -2.25470 & C & -4.04810 & 1.56310 & 15.50840 \\
\hline C & -11.19900 & -2.71550 & -1.68170 & C & -3.16100 & 1.88800 & 16.54390 \\
\hline O & -12.35600 & -2.32970 & -1.78240 & C & -2.35530 & 3.02250 & 16.49280 \\
\hline $\mathrm{O}$ & -9.21920 & -5.51590 & -2.74070 & C & -2.41400 & 3.87790 & 15.39850 \\
\hline C & -11.76370 & -4.73440 & -2.98410 & $\mathrm{H}$ & -3.08520 & 1.24650 & 17.41240 \\
\hline $\mathrm{H}$ & -0.74130 & 4.57870 & -3.66410 & $\mathrm{H}$ & -1.66860 & 3.25740 & 17.29830 \\
\hline $\mathrm{H}$ & -3.45040 & -1.28070 & -2.08790 & C & -1.52910 & 5.06880 & 15.35540 \\
\hline $\mathrm{H}$ & 5.24770 & 2.43890 & -1.64620 & C & -5.75230 & 0.94720 & 13.26990 \\
\hline $\mathrm{H}$ & 2.69050 & -3.47950 & -0.03200 & C & -6.50720 & 0.59820 & 12.12070 \\
\hline $\mathrm{H}$ & -4.89040 & 2.77590 & -5.74510 & C & -7.36550 & -0.53650 & 12.20580 \\
\hline $\mathrm{H}$ & -5.88300 & 1.80760 & -4.61030 & C & -7.40530 & -1.33250 & 13.32180 \\
\hline $\mathrm{H}$ & -5.56110 & 3.54340 & -4.29920 & $\mathrm{H}$ & -8.05610 & -0.77760 & 11.41110 \\
\hline $\mathrm{H}$ & 8.07880 & -1.14870 & -0.63680 & C & -8.34520 & -2.48360 & 13.34250 \\
\hline $\mathrm{H}$ & 7.49030 & -2.24530 & 0.65340 & C & -6.57150 & -1.05820 & 14.44130 \\
\hline $\mathrm{H}$ & 7.45800 & -2.78160 & -1.03300 & C & -5.73850 & 0.09450 & 14.42020 \\
\hline $\mathrm{H}$ & 1.24940 & -4.69910 & -0.34280 & C & -4.91190 & 0.38190 & 15.54730 \\
\hline $\mathrm{H}$ & -0.62350 & -6.24620 & -0.59300 & C & -4.96450 & -0.48850 & 16.64740 \\
\hline $\mathrm{H}$ & -2.96350 & -6.67560 & -0.93940 & C & -5.79670 & -1.60630 & 16.66400 \\
\hline $\mathrm{H}$ & -5.27390 & -5.87560 & -1.15090 & C & -6.60340 & -1.89850 & 15.56990 \\
\hline $\mathrm{H}$ & -6.95330 & -5.04930 & -1.86710 & $\mathrm{H}$ & -4.35240 & -0.29700 & 17.51890 \\
\hline $\mathrm{H}$ & -11.53500 & -0.42280 & -0.45910 & $\mathrm{H}$ & -5.83520 & -2.26370 & 17.52570 \\
\hline $\mathrm{H}$ & -11.45450 & -4.84450 & -4.02590 & C & -7.50540 & -3.07720 & 15.61320 \\
\hline $\mathrm{H}$ & -11.82800 & -5.72950 & -2.53950 & $\mathrm{~N}$ & -1.60750 & 5.86290 & 14.19970 \\
\hline $\mathrm{H}$ & -12.72150 & -4.22370 & -2.92310 & $\mathrm{~N}$ & -8.31130 & -3.29740 & 14.48530 \\
\hline C & -5.90200 & -0.15450 & 1.20730 & $\mathrm{O}$ & -0.76160 & 5.34450 & 16.26790 \\
\hline C & -7.26820 & 0.26240 & 1.24650 & $\mathrm{O}$ & -2.42870 & 6.36870 & 12.13770 \\
\hline C & -7.66390 & 1.37760 & 2.03340 & $\mathrm{O}$ & -7.56600 & -3.82530 & 16.58060 \\
\hline C & -6.64660 & 2.11000 & 2.69640 & $\mathrm{O}$ & -9.11210 & -2.72570 & 12.42070 \\
\hline C & -5.32350 & 1.72400 & 2.65690 & C & -0.73700 & 7.03780 & 14.09570 \\
\hline C & -4.92730 & 0.58420 & 1.91730 & C & -9.21440 & -4.45290 & 14.52390 \\
\hline C & -3.19640 & -0.93470 & 1.15070 & $\mathrm{H}$ & -0.15310 & 7.09940 & 15.01060 \\
\hline C & -3.57400 & 0.17070 & 1.88300 & $\mathrm{H}$ & -0.08230 & 6.93710 & 13.22740 \\
\hline C & -2.54780 & 0.92730 & 2.64420 & $\mathrm{H}$ & -1.34440 & 7.93560 & 13.96400 \\
\hline $\mathrm{N}$ & -2.99120 & 2.05400 & 3.35320 & $\mathrm{H}$ & -8.73360 & -5.25020 & 15.08800 \\
\hline C & -4.31710 & 2.52050 & 3.40720 & $\mathrm{H}$ & -10.15580 & -4.18980 & 15.01670 \\
\hline O & -4.61480 & 3.51720 & 4.05250 & $\mathrm{H}$ & -9.42100 & -4.75910 & 13.50080 \\
\hline O & -1.36390 & 0.61560 & 2.66330 & C & 2.85360 & 3.89800 & -1.64840 \\
\hline C & -1.96180 & 2.78940 & 4.09310 & C & 1.58870 & 4.36510 & -2.09410 \\
\hline $\mathrm{H}$ & -6.88000 & 3.01310 & 3.24080 & C & 2.56360 & 6.62300 & -2.42980 \\
\hline $\mathrm{H}$ & -2.44390 & 3.62970 & 4.58660 & C & 1.41690 & 5.78700 & -2.34420 \\
\hline $\mathrm{H}$ & -1.49130 & 2.13050 & 4.82620 & C & 0.14780 & 6.42420 & -2.47380 \\
\hline $\mathrm{H}$ & -1.18970 & 3.13970 & 3.40480 & C & 0.02770 & 7.71410 & -2.92580 \\
\hline $\mathrm{H}$ & -2.15890 & -1.23060 & 1.19880 & $\mathrm{H}$ & -0.76620 & 5.91720 & -2.20370 \\
\hline C & -9.06010 & 1.80650 & 2.02000 & $\mathrm{C}$ & -1.32900 & 8.28720 & -3.11170 \\
\hline C & -9.92110 & 1.25710 & 1.03570 & C & 1.17940 & 8.49040 & -3.23040 \\
\hline C & -11.11810 & 1.94590 & 0.68320 & C & 2.46070 & 7.96670 & -2.91290 \\
\hline C & -11.41230 & 3.15500 & 1.24220 & C & 3.61400 & 8.79350 & -3.06600 \\
\hline $\mathrm{H}$ & -11.75560 & 1.54960 & -0.09580 & C & 3.44640 & 10.06020 & -3.64690 \\
\hline $\mathrm{H}$ & -12.25200 & 3.74260 & 0.88270 & C & 2.18800 & 10.54730 & -3.99650 \\
\hline C & -9.57840 & 2.87070 & 2.85710 & C & 1.04730 & 9.78620 & -3.76170 \\
\hline C & -10.65200 & 3.64730 & 2.33650 & $\mathrm{H}$ & 4.30500 & 10.69870 & -3.81060 \\
\hline C & -10.97100 & 4.89670 & 2.93070 & $\mathrm{H}$ & 2.07150 & 11.53620 & -4.42630 \\
\hline C & -10.27340 & 5.35620 & 4.00870 & C & -0.29390 & 10.36000 & -4.03940 \\
\hline $\mathrm{H}$ & -11.77760 & 5.48320 & 2.50380 & C & 3.82280 & 6.15220 & -1.94910 \\
\hline
\end{tabular}



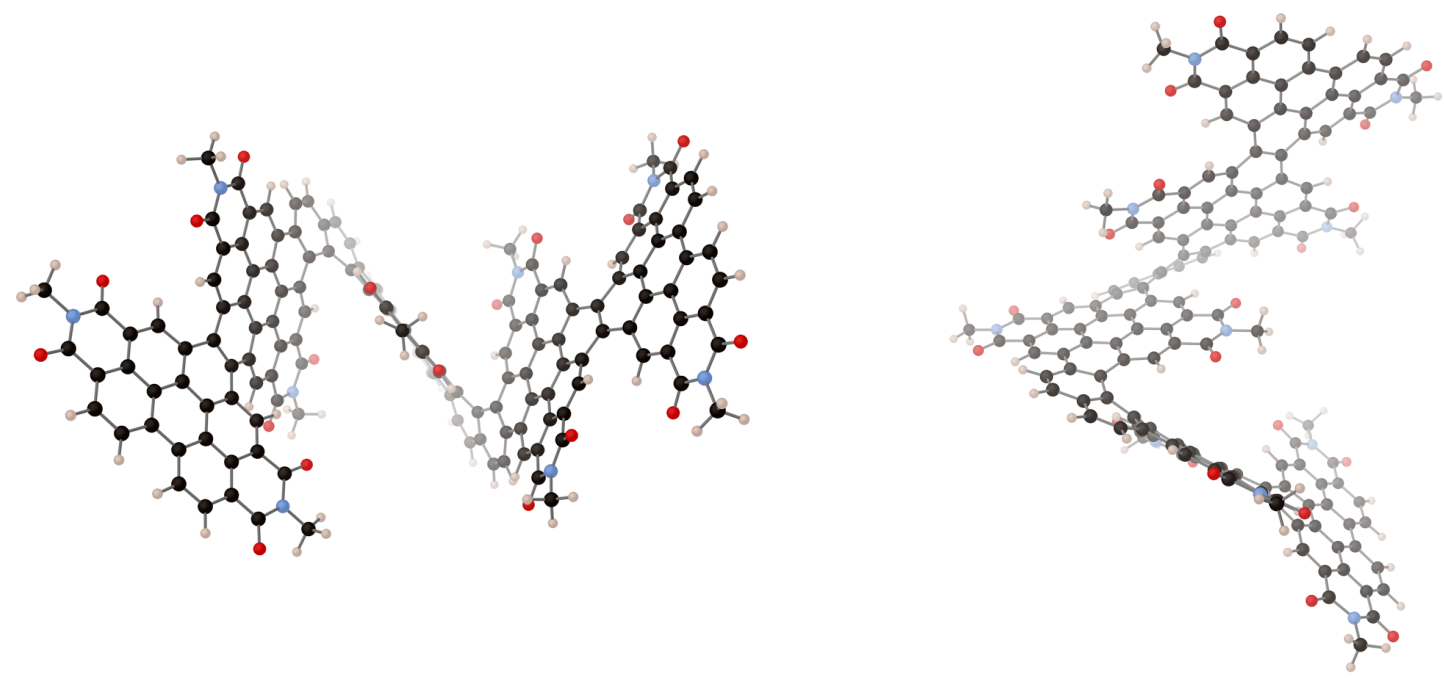

Figure S32. DFT-optimized (B3LYP/6-31G**) structure of PP-M[6][6]-MP from different perspectives (total energy $=-7958.7812$ hartrees).

Cartesian Coordinates of $\boldsymbol{P P}-\boldsymbol{M}$ 6][6]-MP (from left to right: atom; $\mathrm{x}, \mathrm{y}, \mathrm{z}$ in $\AA$ )

\begin{tabular}{|c|c|c|c|c|c|c|c|}
\hline C & 0.37220 & 0.95280 & -3.39020 & $\mathrm{C}$ & -10.70120 & 5.14470 & 2.85360 \\
\hline C & -0.82990 & 0.22380 & -3.13820 & $\mathrm{C}$ & -9.83990 & 5.60410 & 3.80350 \\
\hline C & -2.06010 & 0.74720 & -3.59220 & $\mathrm{H}$ & -11.53150 & 5.75730 & 2.51540 \\
\hline C & -2.09500 & 2.03890 & -4.16460 & $\mathrm{H}$ & -9.96170 & 6.60910 & 4.18560 \\
\hline C & -0.92770 & 2.73830 & -4.39050 & C & -8.85950 & 4.73960 & 4.37120 \\
\hline C & 0.34610 & 2.16400 & -4.13510 & $\mathrm{C}$ & -8.74280 & 3.40430 & 3.89820 \\
\hline C & -0.81560 & -1.01560 & -2.42900 & $\mathrm{C}$ & -7.39090 & 2.86810 & 5.92220 \\
\hline C & -2.01610 & -1.74800 & -2.22050 & $\mathrm{C}$ & -8.12010 & 2.42730 & 4.78190 \\
\hline C & -3.20250 & -1.24570 & -2.81520 & $\mathrm{C}$ & -8.30770 & 1.03090 & 4.61740 \\
\hline C & -3.23960 & -0.01800 & -3.43860 & $\mathrm{C}$ & -7.69260 & 0.11630 & 5.44280 \\
\hline C & 1.59340 & 0.50380 & -2.80580 & $\mathrm{H}$ & -8.96870 & 0.64620 & 3.85400 \\
\hline C & 1.59250 & -0.68690 & -2.02230 & $\mathrm{C}$ & -6.70920 & 1.92820 & 6.75520 \\
\hline C & 0.42380 & -1.49170 & -1.90570 & $\mathrm{C}$ & -6.85240 & 0.54570 & 6.49870 \\
\hline C & 2.77860 & 1.27430 & -2.94380 & $\mathrm{C}$ & -6.20700 & -0.39020 & 7.34090 \\
\hline C & 3.85040 & 0.97140 & -2.05900 & $\mathrm{C}$ & -5.37430 & 0.03400 & 8.35530 \\
\hline C & 3.86320 & -0.18370 & -1.30550 & $\mathrm{C}$ & -5.15090 & 1.41240 & 8.60570 \\
\hline C & 2.76720 & -1.07620 & -1.34110 & $\mathrm{C}$ & -5.90250 & 2.35580 & 7.85390 \\
\hline C & 2.80350 & -2.30850 & -0.64680 & $\mathrm{H}$ & -4.93970 & -0.72800 & 8.98700 \\
\hline C & 1.70510 & -3.14180 & -0.64880 & $\mathrm{C}$ & -8.07000 & 5.19340 & 5.50060 \\
\hline C & 0.48770 & -2.74770 & -1.25800 & $\mathrm{C}$ & -7.31690 & 4.25610 & 6.24820 \\
\hline C & -4.53720 & 0.51190 & -3.92750 & $\mathrm{C}$ & -7.23900 & 6.98620 & 6.92860 \\
\hline $\mathrm{N}$ & -4.52560 & 1.82030 & -4.43590 & $\mathrm{C}$ & -8.01280 & 6.55990 & 5.87210 \\
\hline C & -3.39820 & 2.66000 & -4.51660 & $\mathrm{H}$ & -8.56050 & 7.32020 & 5.33240 \\
\hline C & 5.03850 & -0.48660 & -0.44770 & $\mathrm{C}$ & -6.50280 & 6.05650 & 7.70120 \\
\hline $\mathrm{N}$ & 5.06450 & -1.76070 & 0.14540 & $\mathrm{C}$ & -6.54770 & 4.68120 & 7.37380 \\
\hline C & 4.02160 & -2.70100 & 0.10880 & $\mathrm{C}$ & -5.84910 & 3.74240 & 8.19070 \\
\hline $\mathrm{O}$ & -5.58290 & -0.12140 & -3.86600 & $\mathrm{C}$ & -5.15280 & 4.17180 & 9.35570 \\
\hline $\mathrm{O}$ & -3.49890 & 3.82290 & -4.87850 & $\mathrm{C}$ & -5.05300 & 5.57000 & 9.58510 \\
\hline $\mathrm{O}$ & 4.12120 & -3.77550 & 0.68520 & C & -5.71150 & 6.48480 & 8.79140 \\
\hline $\mathrm{O}$ & 5.95420 & 0.30390 & -0.27360 & $\mathrm{H}$ & -4.42800 & 5.97000 & 10.37010 \\
\hline C & -5.81980 & 2.36770 & -4.85320 & $\mathrm{C}$ & -5.58110 & 7.93530 & 9.08960 \\
\hline C & 6.25570 & -2.10150 & 0.93060 & $\mathrm{~N}$ & -6.35310 & 8.81170 & 8.31190 \\
\hline C & -1.96890 & -3.01310 & -1.49710 & $\mathrm{C}$ & -7.18820 & 8.43700 & 7.24660 \\
\hline C & -0.69850 & -3.57950 & -1.21940 & $\mathrm{C}$ & -6.43860 & -1.84760 & 7.16260 \\
\hline C & -0.58980 & -4.97910 & -0.96820 & $\mathrm{~N}$ & -7.26790 & -2.21730 & 6.08820 \\
\hline C & -1.69250 & -5.78120 & -1.04040 & C & -7.91910 & -1.33270 & 5.21650 \\
\hline C & -2.99290 & -5.21490 & -1.15100 & $\mathrm{O}$ & -4.85550 & 8.37110 & 9.97180 \\
\hline $\mathrm{C}$ & -3.14480 & -3.80180 & -1.17980 & $\mathrm{O}$ & -7.83410 & 9.26640 & 6.62040 \\
\hline C & -4.14350 & -6.04690 & -1.22830 & $\mathrm{O}$ & -5.94200 & -2.68600 & 7.90140 \\
\hline C & -5.39310 & -5.50420 & -1.30950 & $\mathrm{O}$ & -8.64080 & -1.76410 & 4.32690 \\
\hline
\end{tabular}

$\begin{array}{lccc}\mathrm{C} & 2.80220 & 7.93130 & -8.31120 \\ \mathrm{C} & 1.68400 & 7.10760 & -8.24170 \\ \mathrm{H} & 4.82460 & 8.24700 & -7.72320 \\ \mathrm{H} & 2.75920 & 8.81260 & -8.94170 \\ \mathrm{C} & 0.48830 & 7.41690 & -9.06260 \\ \mathrm{C} & 4.06950 & 4.07880 & -5.18220 \\ \mathrm{C} & 4.07760 & 2.89510 & -4.40000 \\ \mathrm{C} & 5.34790 & 2.29570 & -4.14510 \\ \mathrm{C} & 6.52510 & 2.94310 & -4.41920 \\ \mathrm{H} & 5.42570 & 1.29020 & -3.76000 \\ \mathrm{C} & 7.81060 & 2.27030 & -4.09360 \\ \mathrm{C} & 6.51990 & 4.23600 & -5.01140 \\ \mathrm{C} & 5.28610 & 4.78330 & -5.45480 \\ \mathrm{C} & 5.28830 & 6.00700 & -6.18720 \\ \mathrm{C} & 6.50730 & 6.68630 & -6.33930 \\ \mathrm{C} & 7.70700 & 6.16060 & -5.86320 \\ \mathrm{C} & 7.72760 & 4.92520 & -5.22380 \\ \mathrm{H} & 6.53730 & 7.63030 & -6.86880 \\ \mathrm{H} & 8.64470 & 6.68670 & -6.00490 \\ \mathrm{C} & 9.02040 & 4.33300 & -4.79810 \\ \mathrm{~N} & -0.56800 & 6.49400 & -9.01010 \\ \mathrm{~N} & 8.97090 & 3.04610 & -4.23920 \\ \mathrm{O} & 0.41330 & 8.41260 & -9.77020 \\ \mathrm{O} & -1.47880 & 4.49200 & -8.38090 \\ \mathrm{O} & 7.87060 & 1.11410 & -3.70050 \\ \mathrm{O} & 10.08840 & 4.91460 & -4.93480 \\ \mathrm{C} & -1.73470 & 6.76810 & -9.85500 \\ \mathrm{C} & 10.24810 & 2.43560 & -3.85490 \\ \mathrm{H} & -1.89720 & 7.84400 & -9.88210 \\ \mathrm{H} & -1.56340 & 6.41250 & -10.87610 \\ \mathrm{H} & -2.59030 & 6.24510 & -9.43390 \\ \mathrm{H} & 10.72620 & 1.96300 & -4.71880 \\ \mathrm{H} & 10.90440 & 3.21660 & -3.47500 \\ \mathrm{H} & 10.05000 & 1.67950 & -3.09850\end{array}$




\begin{tabular}{|c|c|c|c|c|c|c|c|}
\hline C & -5.58820 & -4.10630 & -1.10550 & $\mathrm{C}$ & -6.24390 & 10.23490 & 8.64870 \\
\hline C & -4.46140 & -3.27560 & -0.88210 & $\mathrm{C}$ & -7.50810 & -3.64390 & 5.85220 \\
\hline C & -6.92650 & -3.55280 & -1.01920 & $\mathrm{H}$ & -6.95650 & 10.77770 & 8.03280 \\
\hline C & -7.10360 & -2.25070 & -0.49120 & $\mathrm{H}$ & -6.45880 & 10.37610 & 9.70930 \\
\hline C & -5.97850 & -1.50710 & -0.01810 & $\mathrm{H}$ & -5.22810 & 10.58790 & 8.45540 \\
\hline C & -4.66200 & -2.01030 & -0.19140 & $\mathrm{H}$ & -7.25120 & -3.89170 & 4.82080 \\
\hline C & -8.06560 & -4.26180 & -1.47610 & $\mathrm{H}$ & -6.89450 & -4.20530 & 6.55200 \\
\hline C & -9.32290 & -3.69790 & -1.44400 & $\mathrm{H}$ & -8.56550 & -3.87420 & 6.00350 \\
\hline C & -9.51590 & -2.40480 & -0.90300 & C & -4.49000 & 3.18350 & 10.19540 \\
\hline C & -8.41000 & -1.69510 & -0.38010 & C & -4.28340 & 1.88240 & 9.67590 \\
\hline C & -10.80110 & -1.81180 & -0.87610 & C & -4.02430 & 3.46520 & 11.54180 \\
\hline C & -10.98080 & -0.56050 & -0.32620 & C & -3.08200 & 2.60220 & 12.16000 \\
\hline C & -9.90540 & 0.14880 & 0.26420 & C & -4.12190 & 4.79300 & 13.59610 \\
\hline C & -8.61720 & -0.43250 & 0.24430 & C & -4.56800 & 4.52540 & 12.32630 \\
\hline C & -10.47970 & -4.45750 & -1.98190 & C & -4.73570 & 5.91930 & 14.35020 \\
\hline $\mathrm{N}$ & -11.72920 & -3.82090 & -1.94200 & $\mathrm{H}$ & -5.39290 & 5.12180 & 11.96400 \\
\hline C & -11.97580 & -2.53530 & -1.43100 & C & -3.08890 & 4.00950 & 14.18240 \\
\hline O & -13.09880 & -2.04910 & -1.44520 & C & -2.57380 & 2.89300 & 13.46830 \\
\hline O & -10.38070 & -5.58580 & -2.44520 & C & -1.55820 & 2.08910 & 14.06770 \\
\hline C & -12.85630 & -4.58450 & -2.48680 & C & -1.10060 & 2.43710 & 15.34880 \\
\hline $\mathrm{H}$ & -1.02760 & 3.74640 & -4.76400 & C & -1.61500 & 3.53100 & 16.04110 \\
\hline $\mathrm{H}$ & -4.12330 & -1.80770 & -2.77530 & C & -2.60740 & 4.31910 & 15.46860 \\
\hline $\mathrm{H}$ & 4.69030 & 1.63930 & -1.94090 & $\mathrm{H}$ & -0.32750 & 1.84930 & 15.82610 \\
\hline $\mathrm{H}$ & 1.78650 & -4.07250 & -0.10090 & $\mathrm{H}$ & -1.25570 & 3.78760 & 17.03160 \\
\hline $\mathrm{H}$ & -5.63150 & 3.25430 & -5.45330 & C & -3.15590 & 5.47060 & 16.22510 \\
\hline $\mathrm{H}$ & -6.35720 & 1.61040 & -5.42320 & C & -2.61430 & 1.44650 & 11.46270 \\
\hline $\mathrm{H}$ & -6.42140 & 2.63750 & -3.98020 & C & -3.20390 & 1.08380 & 10.22340 \\
\hline $\mathrm{H}$ & 7.12260 & -1.63550 & 0.46560 & C & -2.62290 & 0.00170 & 9.49910 \\
\hline $\mathrm{H}$ & 6.16210 & -1.72710 & 1.95490 & C & -1.58740 & -0.73900 & 10.01010 \\
\hline $\mathrm{H}$ & 6.34950 & -3.18480 & 0.95390 & $\mathrm{H}$ & -2.94610 & -0.23460 & 8.49520 \\
\hline $\mathrm{H}$ & 0.38820 & -5.42080 & -0.82250 & C & -1.01940 & -1.84070 & 9.18660 \\
\hline $\mathrm{H}$ & -1.59680 & -6.86130 & -0.97870 & C & -1.06170 & -0.45650 & 11.30270 \\
\hline $\mathrm{H}$ & -4.00690 & -7.12430 & -1.24100 & C & -1.56900 & 0.65220 & 12.03410 \\
\hline $\mathrm{H}$ & -6.24850 & -6.15500 & -1.43400 & C & -1.02900 & 0.94810 & 13.32190 \\
\hline $\mathrm{H}$ & -7.98310 & -5.25220 & -1.90400 & C & -0.01600 & 0.11840 & 13.82640 \\
\hline $\mathrm{H}$ & -11.98900 & -0.16760 & -0.32020 & C & 0.48370 & -0.95630 & 13.09540 \\
\hline $\mathrm{H}$ & -12.66880 & -4.82420 & -3.53570 & C & -0.02900 & -1.24900 & 11.83700 \\
\hline $\mathrm{H}$ & -12.96560 & -5.52250 & -1.93830 & $\mathrm{H}$ & 0.40450 & 0.31030 & 14.80490 \\
\hline $\mathrm{H}$ & -13.75030 & -3.97450 & -2.38600 & $\mathrm{H}$ & 1.27640 & -1.58170 & 13.49100 \\
\hline C & -6.19760 & -0.27130 & 0.66520 & C & 0.52370 & -2.38880 & 11.06760 \\
\hline C & -7.51270 & 0.25910 & 0.83090 & $\mathrm{~N}$ & -4.16820 & 6.21350 & 15.59970 \\
\hline C & -7.72060 & 1.44360 & 1.58970 & $\mathrm{~N}$ & -0.02550 & -2.62110 & 9.79790 \\
\hline C & -6.58090 & 2.15590 & 2.04190 & $\mathrm{O}$ & -2.76350 & 5.77420 & 17.34390 \\
\hline C & -5.30750 & 1.65050 & 1.89100 & $\mathrm{O}$ & -5.67170 & 6.57210 & 13.91100 \\
\hline C & -5.09670 & 0.42430 & 1.21450 & $\mathrm{O}$ & 1.42090 & -3.10380 & 11.49380 \\
\hline C & -3.59310 & -1.30270 & 0.41400 & $\mathrm{O}$ & -1.39590 & -2.07150 & 8.04600 \\
\hline C & -3.79570 & -0.11550 & 1.08120 & C & -4.71090 & 7.34920 & 16.35230 \\
\hline C & -2.62510 & 0.60550 & 1.64020 & C & 0.53920 & -3.74290 & 9.04190 \\
\hline $\mathrm{N}$ & -2.89650 & 1.79020 & 2.34580 & $\mathrm{H}$ & -3.88870 & 7.87780 & 16.83350 \\
\hline C & -4.15870 & 2.39420 & 2.47240 & $\mathrm{H}$ & -5.24330 & 7.99140 & 15.65520 \\
\hline O & -4.28850 & 3.46510 & 3.05170 & $\mathrm{H}$ & -5.39960 & 7.00140 & 17.12780 \\
\hline O & -1.47410 & 0.21210 & 1.50350 & $\mathrm{H}$ & 0.60560 & -4.61450 & 9.69320 \\
\hline C & -1.74790 & 2.49240 & 2.92600 & $\mathrm{H}$ & -0.10890 & -3.93250 & 8.19030 \\
\hline $\mathrm{H}$ & -6.68460 & 3.11110 & 2.53580 & $\mathrm{H}$ & 1.54650 & -3.49740 & 8.69430 \\
\hline $\mathrm{H}$ & -2.10500 & 3.10570 & 3.75020 & C & 2.82020 & 2.31810 & -3.95920 \\
\hline $\mathrm{H}$ & -1.02380 & 1.75310 & 3.26370 & C & 1.60810 & 2.75720 & -4.55930 \\
\hline $\mathrm{H}$ & -1.27090 & 3.13760 & 2.18100 & C & 2.85400 & 4.51570 & -5.79050 \\
\hline $\mathrm{H}$ & -2.58660 & -1.69150 & 0.39090 & C & 1.66420 & 3.76410 & -5.60530 \\
\hline C & -9.07320 & 1.95140 & 1.76710 & $\mathrm{C}$ & 0.59670 & 4.00720 & -6.52250 \\
\hline C & -10.09770 & 1.41770 & 0.94060 & C & 0.60340 & 5.07800 & -7.38080 \\
\hline C & -11.29850 & 2.15740 & 0.74330 & $\mathrm{H}$ & -0.24360 & 3.33270 & -6.59310 \\
\hline C & -11.44900 & 3.38910 & 1.30790 & C & -0.56120 & 5.29390 & -8.28210 \\
\hline $\mathrm{H}$ & -12.05140 & 1.78710 & 0.05940 & C & 1.71070 & 5.97080 & -7.41370 \\
\hline $\mathrm{H}$ & -12.30740 & 4.00810 & 1.06420 & C & 2.87260 & 5.65300 & -6.66050 \\
\hline C & -9.41020 & 3.05260 & 2.65360 & C & 4.04250 & 6.45530 & -6.80970 \\
\hline C & -10.51600 & 3.86480 & 2.26750 & $\mathrm{C}$ & 3.96180 & 7.60270 & -7.61290 \\
\hline
\end{tabular}



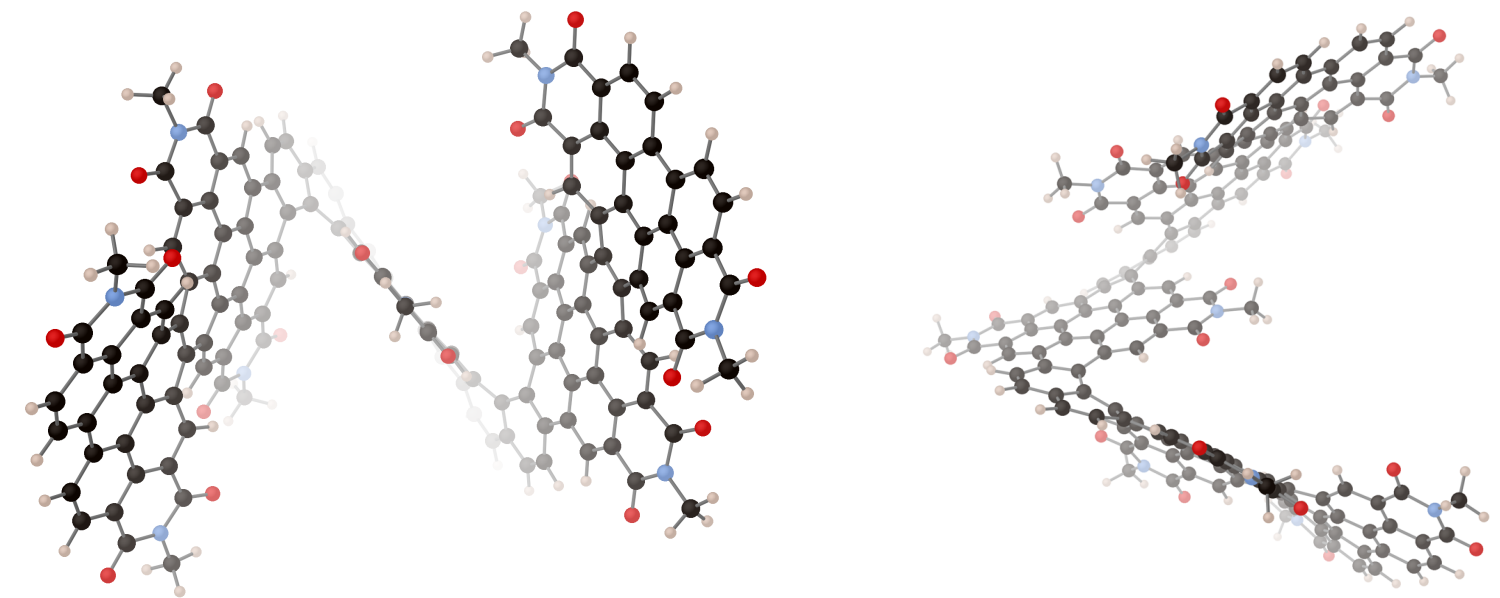

Figure S33. DFT-optimized (B3LYP/6-31G*) structure of $P M-M[6][6]-P M$ from different perspectives (total energy $=-7958.7747$ hartrees).

Cartesian Coordinates of $\boldsymbol{P M}-\boldsymbol{M}[\mathbf{6}][6]-P M$ (from left to right: atom; $x, y, z$ in $\AA$ )

\begin{tabular}{|c|c|c|c|c|c|c|c|}
\hline C & 0.58540 & 2.03010 & -1.87520 & $\mathrm{H}$ & -11.70970 & 5.43310 & 2.31640 \\
\hline C & -0.48650 & 1.10170 & -2.03420 & $\mathrm{H}$ & -10.32270 & 6.26270 & 4.15430 \\
\hline C & -1.70000 & 1.53480 & -2.61330 & C & -9.19450 & 4.41610 & 4.38590 \\
\hline C & -1.80750 & 2.86290 & -3.08540 & C & -9.01120 & 3.10260 & 3.88740 \\
\hline C & -0.78610 & 3.76550 & -2.87560 & C & -7.85540 & 2.50250 & 6.00910 \\
\hline C & 0.40090 & 3.40380 & -2.18440 & $\mathrm{C}$ & -8.47130 & 2.09560 & 4.79400 \\
\hline C & -0.34350 & -0.26280 & -1.64200 & $\mathrm{C}$ & -8.68780 & 0.70760 & 4.59000 \\
\hline C & -1.45550 & -1.14370 & -1.68920 & $\mathrm{C}$ & -8.32260 & -0.22760 & 5.53330 \\
\hline C & -2.63090 & -0.68560 & -2.33930 & $\mathrm{H}$ & -9.21980 & 0.35020 & 3.72050 \\
\hline C & -2.75300 & 0.60630 & -2.80160 & C & -7.37050 & 1.53240 & 6.94040 \\
\hline C & 1.88300 & 1.54580 & -1.52990 & $\mathrm{C}$ & -7.65300 & 0.16540 & 6.71610 \\
\hline C & 2.06470 & 0.14800 & -1.31730 & $\mathrm{C}$ & -7.37230 & -0.77050 & 7.73590 \\
\hline C & 0.95260 & -0.74450 & -1.28620 & $\mathrm{C}$ & -6.71570 & -0.38200 & 8.88240 \\
\hline C & 3.00020 & 2.42610 & -1.54820 & C & -6.23320 & 0.94100 & 9.06530 \\
\hline C & 4.29020 & 1.83090 & -1.60400 & $\mathrm{C}$ & -6.67010 & 1.92050 & 8.12610 \\
\hline C & 4.47950 & 0.48010 & -1.40040 & $\mathrm{H}$ & -6.61980 & -1.13130 & 9.65150 \\
\hline C & 3.37270 & -0.37210 & -1.18980 & $\mathrm{C}$ & -8.49190 & 4.84340 & 5.58110 \\
\hline C & 3.55470 & -1.75350 & -0.94830 & $\mathrm{C}$ & -7.79980 & 3.88520 & 6.36470 \\
\hline C & 2.47110 & -2.59720 & -0.85390 & $\mathrm{C}$ & -7.86570 & 6.58150 & 7.16530 \\
\hline C & 1.14750 & -2.12500 & -1.03870 & C & -8.48710 & 6.19560 & 5.99830 \\
\hline C & -3.99920 & 1.01240 & -3.50380 & $\mathrm{H}$ & -8.96800 & 6.97180 & 5.41750 \\
\hline$N$ & -4.03710 & 2.33310 & -3.98250 & $\mathrm{C}$ & -7.21110 & 5.62820 & 7.98040 \\
\hline C & -3.01460 & 3.28520 & -3.84180 & C & -7.15670 & 4.27310 & 7.57690 \\
\hline C & 5.86020 & -0.07560 & -1.42230 & $\mathrm{C}$ & -6.53040 & 3.30950 & 8.42700 \\
\hline $\mathrm{N}$ & 5.98610 & -1.43630 & -1.08610 & $\mathrm{C}$ & -5.90820 & 3.71480 & 9.63840 \\
\hline C & 4.92370 & -2.31470 & -0.83330 & C & -6.08340 & 5.06360 & 10.04820 \\
\hline $\mathrm{O}$ & -4.94160 & 0.24770 & -3.66460 & C & -6.69340 & 5.99800 & 9.24230 \\
\hline $\mathrm{O}$ & -3.14100 & 4.39980 & -4.32790 & $\mathrm{H}$ & -5.78660 & 5.39640 & 11.03100 \\
\hline $\mathrm{O}$ & 5.14300 & -3.48690 & -0.55860 & C & -6.83860 & 7.39370 & 9.73140 \\
\hline $\mathrm{O}$ & 6.83650 & 0.60520 & -1.69920 & $\mathrm{~N}$ & -7.40640 & 8.31620 & 8.84120 \\
\hline C & -5.23120 & 2.78120 & -4.70570 & $\mathrm{C}$ & -7.93320 & 8.00680 & 7.57670 \\
\hline C & 7.33050 & -2.01810 & -1.01670 & $\mathrm{C}$ & -7.82580 & -2.18030 & 7.60900 \\
\hline C & -1.31460 & -2.50090 & -1.17900 & $\mathrm{~N}$ & -8.41620 & -2.53330 & 6.38150 \\
\hline C & -0.00010 & -3.01190 & -1.00500 & C & -8.68780 & -1.65300 & 5.32480 \\
\hline C & 0.19260 & -4.41730 & -0.86340 & $\mathrm{O}$ & -6.47660 & 7.75190 & 10.84320 \\
\hline C & -0.85750 & -5.27540 & -0.99040 & $\mathrm{O}$ & -8.43720 & 8.86670 & 6.86780 \\
\hline C & -2.18890 & -4.79060 & -1.07100 & $\mathrm{O}$ & -7.69030 & -2.99740 & 8.50780 \\
\hline C & -2.44330 & -3.39340 & -0.97280 & $\mathrm{O}$ & -9.21710 & -2.06360 & 4.30040 \\
\hline C & -3.26110 & -5.70270 & -1.25180 & C & -7.48190 & 9.70190 & 9.31460 \\
\hline C & -4.54370 & -5.25340 & -1.34040 & C & -8.82390 & -3.92690 & 6.17780 \\
\hline C & -4.85470 & -3.89730 & -1.04130 & $\mathrm{H}$ & -7.90590 & 10.30360 & 8.51460 \\
\hline C & -3.81390 & -2.99800 & -0.69090 & $\mathrm{H}$ & -8.10820 & 9.75620 & 10.20790 \\
\hline
\end{tabular}




\begin{tabular}{|c|c|c|c|c|c|c|c|}
\hline C & -6.23460 & -3.45370 & -1.05050 & $\mathrm{H}$ & -6.48210 & 10.05430 & 9.57620 \\
\hline C & -6.56040 & -2.20210 & -0.48220 & $\mathrm{H}$ & -8.33220 & -4.32720 & 5.28900 \\
\hline C & -5.54390 & -1.41630 & 0.14400 & $\mathrm{H}$ & -8.53700 & -4.48970 & 7.06270 \\
\hline C & -4.17860 & -1.80370 & 0.06250 & $\mathrm{H}$ & -9.90420 & -3.98090 & 6.02480 \\
\hline C & -7.26290 & -4.21560 & -1.66050 & C & -5.20940 & 2.72910 & 10.44730 \\
\hline C & -8.55410 & -3.74180 & -1.74220 & C & -5.39550 & 1.34510 & 10.18820 \\
\hline C & -8.89590 & -2.49390 & -1.16990 & C & -4.36310 & 3.13740 & 11.55270 \\
\hline C & -7.90660 & -1.74020 & -0.49590 & C & -4.07250 & 2.20260 & 12.57490 \\
\hline C & -10.21610 & -1.99080 & -1.25780 & C & -3.13640 & 4.84550 & 12.80170 \\
\hline C & -10.54120 & -0.78120 & -0.68200 & C & -3.76800 & 4.43090 & 11.65520 \\
\hline C & -9.58890 & -0.03330 & 0.05440 & C & -2.54530 & 6.20860 & 12.85960 \\
\hline C & -8.26970 & -0.53280 & 0.16240 & $\mathrm{H}$ & -3.76590 & 5.11230 & 10.81510 \\
\hline C & -9.59020 & -4.54990 & -2.43640 & C & -3.04000 & 3.97740 & 13.92610 \\
\hline $\mathrm{N}$ & -10.88030 & -4.00340 & -2.50080 & C & -3.46060 & 2.62670 & 13.79660 \\
\hline C & -11.27050 & -2.76330 & -1.96680 & C & -3.25170 & 1.71660 & 14.87420 \\
\hline O & -12.41760 & -2.35330 & -2.08230 & C & -2.71780 & 2.21940 & 16.06990 \\
\hline O & -9.36000 & -5.64290 & -2.93640 & C & -2.33630 & 3.55330 & 16.19930 \\
\hline C & -11.88740 & -4.81370 & -3.19360 & C & -2.46590 & 4.43050 & 15.12770 \\
\hline $\mathrm{H}$ & -0.90960 & 4.74950 & -3.30350 & $\mathrm{H}$ & -2.56380 & 1.55890 & 16.91390 \\
\hline $\mathrm{H}$ & -3.45820 & -1.35660 & -2.51950 & $\mathrm{H}$ & -1.90500 & 3.92370 & 17.12270 \\
\hline $\mathrm{H}$ & 5.16980 & 2.41240 & -1.83270 & C & -1.96810 & 5.82240 & 15.25490 \\
\hline $\mathrm{H}$ & 2.68510 & -3.63880 & -0.66020 & C & -4.29640 & 0.81250 & 12.34470 \\
\hline $\mathrm{H}$ & -4.96080 & 3.07370 & -5.72270 & C & -4.77610 & 0.37730 & 11.08140 \\
\hline $\mathrm{H}$ & -5.93930 & 1.95650 & -4.72080 & C & -4.59630 & -1.00400 & 10.76980 \\
\hline $\mathrm{H}$ & -5.66150 & 3.65040 & -4.20400 & C & -4.25110 & -1.92510 & 11.72780 \\
\hline $\mathrm{H}$ & 8.04630 & -1.22590 & -1.22070 & $\mathrm{H}$ & -4.69940 & -1.37070 & 9.75960 \\
\hline $\mathrm{H}$ & 7.49530 & -2.44280 & -0.02440 & C & -4.13600 & -3.35730 & 11.34530 \\
\hline $\mathrm{H}$ & 7.42740 & -2.81980 & -1.75230 & C & -4.00220 & -1.51620 & 13.06770 \\
\hline $\mathrm{H}$ & 1.18780 & -4.82060 & -0.73710 & C & -3.94140 & -0.12910 & 13.36100 \\
\hline $\mathrm{H}$ & -0.69430 & -6.34930 & -0.99970 & C & -3.52470 & 0.29610 & 14.65800 \\
\hline $\mathrm{H}$ & -3.03500 & -6.75980 & -1.35680 & C & -3.32410 & -0.68280 & 15.64350 \\
\hline $\mathrm{H}$ & -5.34120 & -5.95510 & -1.54800 & C & -3.43740 & -2.04280 & 15.35870 \\
\hline $\mathrm{H}$ & -7.06230 & -5.17180 & -2.12440 & C & -3.73970 & -2.46760 & 14.06920 \\
\hline $\mathrm{H}$ & -11.56870 & -0.45310 & -0.77560 & $\mathrm{H}$ & -3.04780 & -0.39090 & 16.64880 \\
\hline $\mathrm{H}$ & -11.58550 & -4.97390 & -4.23090 & $\mathrm{H}$ & -3.26140 & -2.79170 & 16.12330 \\
\hline $\mathrm{H}$ & -11.97420 & -5.78790 & -2.70820 & C & -3.76060 & -3.91370 & 13.74960 \\
\hline $\mathrm{H}$ & -12.83190 & -4.27710 & -3.14980 & $\mathrm{~N}$ & -2.03040 & 6.61660 & 14.09770 \\
\hline C & -5.92610 & -0.25330 & 0.87810 & $\mathrm{~N}$ & -3.94690 & -4.26290 & 12.40340 \\
\hline C & -7.28650 & 0.17830 & 0.91860 & $\mathrm{O}$ & -1.50600 & 6.25460 & 16.30180 \\
\hline C & -7.65820 & 1.30800 & 1.69320 & $\mathrm{O}$ & -2.50850 & 6.96210 & 11.89740 \\
\hline C & -6.62790 & 2.04540 & 2.33100 & $\mathrm{O}$ & -3.60160 & -4.78310 & 14.59540 \\
\hline C & -5.31260 & 1.64100 & 2.29570 & $\mathrm{O}$ & -4.22040 & -3.73790 & 10.18670 \\
\hline C & -4.94150 & 0.47900 & 1.58050 & C & -1.49830 & 7.98160 & 14.15550 \\
\hline C & -3.23430 & -1.07050 & 0.82570 & C & -3.91610 & -5.69730 & 12.09990 \\
\hline C & -3.59710 & 0.03910 & 1.55720 & $\mathrm{H}$ & -1.15850 & 8.16190 & 15.17250 \\
\hline C & -2.55750 & 0.78430 & 2.31110 & $\mathrm{H}$ & -0.67110 & 8.08710 & 13.45000 \\
\hline $\mathrm{N}$ & -2.97790 & 1.93150 & 3.00030 & $\mathrm{H}$ & -2.27860 & 8.69280 & 13.87720 \\
\hline C & -4.29280 & 2.43000 & 3.03670 & $\mathrm{H}$ & -2.94170 & -6.11450 & 12.36430 \\
\hline O & -4.57140 & 3.44420 & 3.66280 & $\mathrm{H}$ & -4.67900 & -6.21210 & 12.68700 \\
\hline O & -1.38040 & 0.44780 & 2.33880 & $\mathrm{H}$ & -4.10360 & -5.81550 & 11.03570 \\
\hline C & -1.93570 & 2.65460 & 3.73400 & C & 2.76040 & 3.86440 & -1.55260 \\
\hline $\mathrm{H}$ & -6.84370 & 2.96450 & 2.85340 & C & 1.45620 & 4.34720 & -1.84300 \\
\hline $\mathrm{H}$ & -2.40040 & 3.51360 & 4.21170 & C & 2.28950 & 6.68080 & -1.79120 \\
\hline $\mathrm{H}$ & -1.48390 & 1.99650 & 4.47950 & C & 1.19940 & 5.77390 & -1.79160 \\
\hline $\mathrm{H}$ & -1.15190 & 2.97630 & 3.04470 & C & -0.11260 & 6.32540 & -1.67830 \\
\hline $\mathrm{H}$ & -2.20190 & -1.38150 & 0.87790 & C & -0.34720 & 7.67270 & -1.78530 \\
\hline C & -9.05090 & 1.73460 & 1.70990 & $\mathrm{H}$ & -0.96580 & 5.69570 & -1.47190 \\
\hline C & -9.93880 & 1.19130 & 0.74780 & C & -1.74160 & 8.17950 & -1.67890 \\
\hline C & -11.15260 & 1.87830 & 0.45270 & C & 0.73220 & 8.57920 & -1.98540 \\
\hline C & -11.42870 & 3.08180 & 1.03550 & C & 2.06610 & 8.08810 & -1.93670 \\
\hline $\mathrm{H}$ & -11.81590 & 1.49260 & -0.31030 & C & 3.15360 & 9.00800 & -2.01020 \\
\hline $\mathrm{H}$ & -12.28560 & 3.66730 & 0.71530 & C & 2.86170 & 10.36360 & -2.22160 \\
\hline C & -9.54020 & 2.78690 & 2.57580 & C & 1.55140 & 10.83040 & -2.30160 \\
\hline C & -10.62940 & 3.56970 & 2.10430 & C & 0.48190 & 9.95290 & -2.15870 \\
\hline C & -10.90220 & 4.82460 & 2.71280 & $\mathrm{H}$ & 3.66640 & 11.08390 & -2.29880 \\
\hline C & -10.13760 & 5.27610 & 3.74930 & $\mathrm{H}$ & 1.34160 & 11.88440 & -2.44660 \\
\hline
\end{tabular}



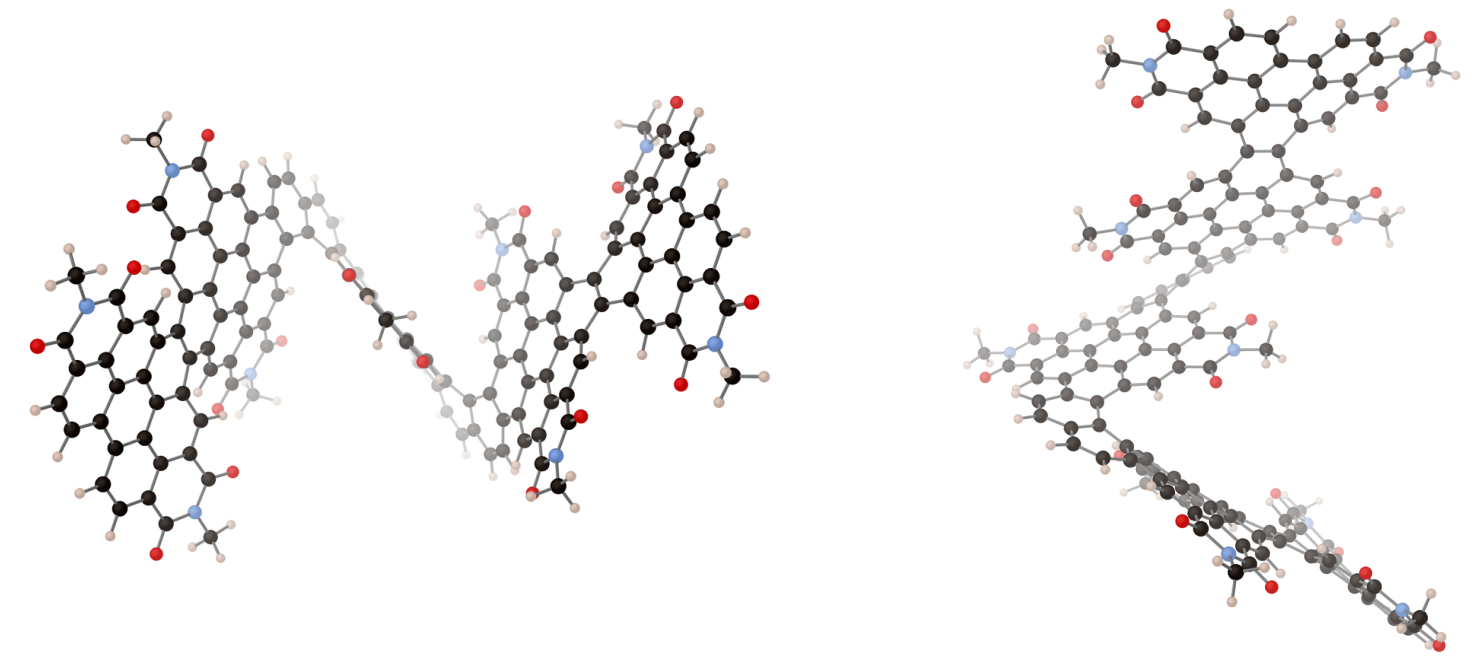

Figure S34. DFT-optimized (B3LYP/6-31G**) structure of $P \boldsymbol{M}-\boldsymbol{M}[\mathbf{6}][\mathbf{6}]-\mathbf{M P}$ from different perspectives (total energy $=-7958.7713$ hartrees).

Cartesian Coordinates of $\boldsymbol{P M - M [ 6 ] [ 6 ] - M P ~ ( f r o m ~ l e f t ~ t o ~ r i g h t : ~ a t o m ; ~ x , ~ y , ~ z ~ i n ~} \AA$ )

\begin{tabular}{|c|c|c|c|c|c|c|c|c|c|c|c|}
\hline $\mathrm{C}$ & 1.01650 & 1.81190 & -2.49860 & $\mathrm{C}$ & -10.31540 & 5.36570 & 2.94760 & C & 3.44900 & 9.26600 & -6.63320 \\
\hline $\mathrm{C}$ & -0.17490 & 1.03310 & -2.38360 & C & -9.61020 & 5.70820 & 4.06210 & C & 2.37250 & 8.38960 & -6.72000 \\
\hline C & -1.39720 & 1.57190 & -2.84290 & $\mathrm{H}$ & -11.04940 & 6.04790 & 2.52880 & $\mathrm{H}$ & 5.40260 & 9.62570 & -5.86150 \\
\hline C & -1.43980 & 2.91190 & -3.29310 & $\mathrm{H}$ & -9.75880 & 6.68510 & 4.50310 & $\mathrm{H}$ & 3.40860 & 10.19170 & -7.19670 \\
\hline C & -0.28350 & 3.65910 & -3.38600 & C & -8.77700 & 4.75340 & 4.71560 & C & 1.23730 & 8.71080 & -7.62230 \\
\hline C & 0.99210 & 3.08860 & -3.12380 & C & -8.63770 & 3.45490 & 4.15520 & C & 4.68960 & 5.20830 & -3.76550 \\
\hline C & -0.15650 & -0.27560 & -1.81210 & C & -7.71460 & 2.66740 & 6.32920 & C & 4.70840 & 3.95150 & -3.10420 \\
\hline C & -1.34110 & -1.05990 & -1.74750 & C & -8.24200 & 2.36780 & 5.04260 & $\mathrm{C}$ & 5.98940 & 3.38740 & -2.82300 \\
\hline C & -2.51380 & -0.52310 & -2.34000 & C & -8.53130 & 1.01000 & 4.74480 & C & 7.14490 & 4.12020 & -2.92460 \\
\hline C & -2.56280 & 0.76820 & -2.81980 & C & -8.25970 & -0.00060 & 5.64000 & $\mathrm{H}$ & 6.09420 & 2.35700 & -2.51820 \\
\hline C & 2.21950 & 1.33890 & -1.89640 & $\mathrm{H}$ & -9.06000 & 0.73960 & 3.84130 & C & 8.43400 & 3.48800 & -2.52420 \\
\hline C & 2.21450 & 0.07590 & -1.23660 & C & -7.32370 & 1.61680 & 7.21540 & C & 7.11010 & 5.46390 & -3.39070 \\
\hline C & 1.06840 & -0.76810 & -1.27000 & C & -7.63000 & 0.27820 & 6.87610 & C & 5.88330 & 5.99210 & -3.87800 \\
\hline C & 3.38950 & 2.14590 & -1.89830 & C & -7.41350 & -0.74570 & 7.82680 & C & 5.87110 & 7.28120 & -4.48810 \\
\hline C & 4.41740 & 1.78730 & -0.98330 & C & -6.80920 & -0.46320 & 9.03270 & C & 7.05410 & 8.03610 & -4.46770 \\
\hline C & 4.43350 & 0.55780 & -0.35910 & C & -6.32430 & 0.83570 & 9.34070 & C & 8.24300 & 7.52420 & -3.95060 \\
\hline C & 3.36410 & -0.35020 & -0.53420 & C & -6.70610 & 1.89610 & 8.47410 & C & 8.28830 & 6.23030 & -3.44230 \\
\hline C & 3.39700 & -1.64010 & 0.04690 & $\mathrm{H}$ & -6.73860 & -1.27360 & 9.74230 & $\mathrm{H}$ & 7.06560 & 9.03420 & -4.88800 \\
\hline $\mathrm{C}$ & 2.33270 & -2.50160 & -0.10970 & C & -8.20430 & 5.06070 & 6.01300 & $\mathrm{H}$ & 9.15740 & 8.10740 & -3.96400 \\
\hline C & 1.14760 & -2.08760 & -0.76840 & C & -7.64550 & 4.01760 & 6.79260 & $\mathrm{C}$ & 9.58140 & 5.65570 & -2.99130 \\
\hline C & -3.86400 & 1.31640 & -3.29250 & C & -7.76600 & 6.64000 & 7.81640 & $\mathrm{~N}$ & 0.23160 & 7.73490 & -7.73030 \\
\hline $\mathrm{N}$ & -3.85870 & 2.66840 & -3.67800 & $\mathrm{C}$ & -8.21590 & 6.37500 & 6.54330 & $\mathrm{~N}$ & 9.56760 & 4.31670 & -2.57300 \\
\hline $\mathrm{C}$ & -2.73870 & 3.51760 & -3.69110 & $\mathrm{H}$ & -8.60800 & 7.21210 & 5.98080 & $\mathrm{O}$ & 1.18300 & 9.75530 & -8.25590 \\
\hline C & 5.58590 & 0.20270 & 0.51340 & C & -7.23600 & 5.60170 & 8.61930 & $\mathrm{O}$ & -0.71880 & 5.73020 & -7.21530 \\
\hline $\mathrm{N}$ & 5.58050 & -1.10210 & 1.04300 & C & -7.13570 & 4.28960 & 8.09920 & $\mathrm{O}$ & 8.50940 & 2.32230 & -2.16430 \\
\hline C & 4.57300 & -2.05960 & 0.85540 & C & -6.60260 & 3.24860 & 8.92120 & O & 10.62710 & 6.29060 & -2.99080 \\
\hline $\mathrm{O}$ & -4.89000 & 0.64910 & -3.31420 & C & -6.11050 & 3.53380 & 10.22700 & C & -0.89500 & 7.98670 & -8.63470 \\
\hline $\mathrm{O}$ & -2.85710 & 4.68620 & -4.02820 & C & -6.38330 & 4.82590 & 10.75130 & C & 10.85790 & 3.75760 & -2.15600 \\
\hline $\mathrm{O}$ & 4.67490 & -3.17000 & 1.35890 & $\mathrm{C}$ & -6.88510 & 5.84060 & 9.96670 & $\mathrm{H}$ & -0.73230 & 8.95430 & -9.10170 \\
\hline $\mathrm{O}$ & 6.49370 & 0.98490 & 0.75140 & $\mathrm{H}$ & -6.21650 & 5.06030 & 11.79100 & $\mathrm{H}$ & -0.94690 & 7.19780 & -9.38750 \\
\hline C & -5.11990 & 3.27410 & -4.11700 & $\mathrm{C}$ & -7.07620 & 7.19080 & 10.56230 & $\mathrm{H}$ & -1.83020 & 7.98200 & -8.07140 \\
\hline C & 6.71430 & -1.52010 & 1.87440 & $\mathrm{~N}$ & -7.53610 & 8.19890 & 9.70050 & $\mathrm{H}$ & 11.56290 & 3.78780 & -2.98950 \\
\hline C & -1.28080 & -2.40120 & -1.17400 & C & -7.89180 & 8.02270 & 8.35380 & $\mathrm{H}$ & 11.27040 & 4.35190 & -1.33880 \\
\hline C & -0.00490 & -2.95480 & -0.89460 & C & -7.88110 & -2.13480 & 7.56260 & $\mathrm{H}$ & 10.68500 & 2.73300 & -1.83780 \\
\hline C & 0.14510 & -4.36830 & -0.80270 & $\mathrm{~N}$ & -8.49110 & -2.35500 & 6.31400 & & & & \\
\hline C & -0.92200 & -5.19220 & -1.00910 & C & -8.69680 & -1.38550 & 5.32270 & & & & \\
\hline C & -2.23680 & -4.66230 & -1.10810 & $\mathrm{O}$ & -6.84060 & 7.44410 & 11.73410 & & & & \\
\hline C & -2.43990 & -3.25860 & -0.99040 & $\mathrm{O}$ & -8.30920 & 8.95080 & 7.67590 & & & & \\
\hline C & -3.35260 & -5.51880 & -1.32140 & $\mathrm{O}$ & -7.75230 & -3.03690 & 8.37690 & & & & \\
\hline C & -4.61700 & -5.00970 & -1.40300 & $\mathrm{O}$ & -9.23360 & -1.68900 & 4.26630 & & & & \\
\hline C & -4.87250 & -3.64720 & -1.07450 & C & -7.66500 & 9.53540 & 10.29080 & & & & \\
\hline
\end{tabular}




\begin{tabular}{|c|c|c|c|c|c|c|c|}
\hline C & -3.78710 & -2.81600 & -0.69420 & $\mathrm{C}$ & -8.96470 & -3.70620 & 5.99910 \\
\hline C & -6.22360 & -3.11780 & -1.06210 & $\mathrm{H}$ & -8.00340 & 10.21020 & 9.50910 \\
\hline C & -6.46940 & -1.87270 & -0.43410 & $\mathrm{H}$ & -8.38180 & 9.51060 & 11.11420 \\
\hline C & -5.40540 & -1.17120 & 0.21510 & $\mathrm{H}$ & -6.70040 & 9.85590 & 10.68880 \\
\hline C & -4.06940 & -1.63750 & 0.11050 & $\mathrm{H}$ & -8.44810 & -4.08530 & 5.11520 \\
\hline C & -7.30190 & -3.78080 & -1.70160 & $\mathrm{H}$ & -8.75940 & -4.33610 & 6.86070 \\
\hline C & -8.56470 & -3.22700 & -1.73740 & $\mathrm{H}$ & -10.03490 & -3.68120 & 5.78450 \\
\hline C & -8.82660 & -1.98970 & -1.10020 & C & -5.44570 & 2.48160 & 10.98660 \\
\hline C & -7.78530 & -1.32870 & -0.40830 & C & -5.54300 & 1.13980 & 10.53190 \\
\hline C & -10.11220 & -1.39720 & -1.15570 & C & -4.70140 & 2.76140 & 12.20480 \\
\hline C & -10.35310 & -0.18630 & -0.54000 & C & -4.35250 & 1.68650 & 13.06710 \\
\hline C & -9.34170 & 0.47900 & 0.19680 & C & -3.75480 & 4.32420 & 13.83520 \\
\hline C & -8.06330 & -0.11830 & 0.28840 & C & -4.25170 & 4.06350 & 12.58250 \\
\hline C & -9.65320 & -3.93140 & -2.46610 & C & -3.40530 & 5.72460 & 14.19970 \\
\hline $\mathrm{N}$ & -10.91110 & -3.30970 & -2.47630 & $\mathrm{H}$ & -4.29330 & 4.89410 & 11.89370 \\
\hline C & -11.22390 & -2.07820 & -1.87360 & C & -3.59840 & 3.27760 & 14.78630 \\
\hline O & -12.34820 & -1.60150 & -1.94570 & C & -3.83560 & 1.93690 & 14.37920 \\
\hline O & -9.49170 & -5.00070 & -3.03850 & C & -3.53440 & 0.86820 & 15.27570 \\
\hline C & -11.97360 & -4.02700 & -3.18830 & C & -3.11690 & 1.19390 & 16.57470 \\
\hline $\mathrm{H}$ & -0.38990 & 4.69360 & -3.68020 & C & -2.93110 & 2.51390 & 16.97930 \\
\hline $\mathrm{H}$ & -3.41730 & -1.11010 & -2.40930 & C & -3.14130 & 3.55900 & 16.08620 \\
\hline $\mathrm{H}$ & 5.23450 & 2.45630 & -0.76020 & $\mathrm{H}$ & -2.91070 & 0.40640 & 17.28890 \\
\hline $\mathrm{H}$ & 2.40930 & -3.47820 & 0.35270 & $\mathrm{H}$ & -2.59340 & 2.75020 & 17.98240 \\
\hline $\mathrm{H}$ & -5.02190 & 3.63040 & -5.14450 & C & -2.85010 & 4.95340 & 16.50730 \\
\hline $\mathrm{H}$ & -5.89280 & 2.51330 & -4.04750 & C & -4.43760 & 0.34060 & 12.59820 \\
\hline $\mathrm{H}$ & -5.36030 & 4.12900 & -3.48200 & C & -4.87710 & 0.08670 & 11.27490 \\
\hline $\mathrm{H}$ & 7.41490 & -0.69060 & 1.91760 & C & -4.60460 & -1.20350 & 10.72880 \\
\hline $\mathrm{H}$ & 6.36470 & -1.78080 & 2.87550 & C & -4.17710 & -2.24540 & 11.51120 \\
\hline $\mathrm{H}$ & 7.18590 & -2.40240 & 1.43760 & $\mathrm{H}$ & -4.72910 & -1.40280 & 9.67490 \\
\hline $\mathrm{H}$ & 1.13390 & -4.79200 & -0.67360 & C & -4.00290 & -3.58770 & 10.88780 \\
\hline $\mathrm{H}$ & -0.78760 & -6.26870 & -1.06270 & C & -3.92290 & -2.04810 & 12.89740 \\
\hline $\mathrm{H}$ & -3.17650 & -6.58310 & -1.44640 & C & -3.99730 & -0.73480 & 13.43520 \\
\hline $\mathrm{H}$ & -5.44660 & -5.66950 & -1.62720 & C & -3.61270 & -0.51000 & 14.78950 \\
\hline $\mathrm{H}$ & -7.15800 & -4.71630 & -2.22780 & C & -3.26270 & -1.62040 & 15.57390 \\
\hline $\mathrm{H}$ & -11.35890 & 0.21080 & -0.60290 & C & -3.22880 & -2.90860 & 15.04490 \\
\hline $\mathrm{H}$ & -11.71140 & -4.13030 & -4.24330 & C & -3.53090 & -3.12820 & 13.70640 \\
\hline $\mathrm{H}$ & -12.08890 & -5.02700 & -2.76630 & $\mathrm{H}$ & -2.98670 & -1.48390 & 16.61180 \\
\hline $\mathrm{H}$ & -12.88990 & -3.45330 & -3.07770 & $\mathrm{H}$ & -2.93830 & -3.75710 & 15.65490 \\
\hline C & -5.70740 & -0.01600 & 0.99810 & C & -3.40420 & -4.49170 & 13.13500 \\
\hline C & -7.03570 & 0.50270 & 1.06480 & $\mathrm{~N}$ & -3.00080 & 5.94570 & 15.52370 \\
\hline C & -7.33440 & 1.61520 & 1.89660 & $\mathrm{~N}$ & -3.64520 & -4.62860 & 11.76040 \\
\hline C & -6.25840 & 2.25660 & 2.56000 & $\mathrm{O}$ & -2.48680 & 5.22890 & 17.64200 \\
\hline C & -4.97200 & 1.76600 & 2.50100 & $\mathrm{O}$ & -3.47270 & 6.65650 & 13.41180 \\
\hline C & -4.67430 & 0.62030 & 1.72470 & $\mathrm{O}$ & -3.09220 & -5.46530 & 13.80600 \\
\hline C & -3.07120 & -0.99420 & 0.88690 & $\mathrm{O}$ & -4.17030 & -3.78520 & 9.69320 \\
\hline C & -3.35750 & 0.10510 & 1.66500 & C & -2.70740 & 7.33750 & 15.88090 \\
\hline C & -2.26570 & 0.75780 & 2.43520 & C & -3.50190 & -5.98110 & 11.21280 \\
\hline $\mathrm{N}$ & -2.61380 & 1.89750 & 3.17680 & $\mathrm{H}$ & -2.44000 & 7.35790 & 16.93410 \\
\hline C & -3.89970 & 2.46290 & 3.26080 & $\mathrm{H}$ & -1.88550 & 7.71330 & 15.26820 \\
\hline $\mathrm{O}$ & -4.11250 & 3.46370 & 3.93220 & $\mathrm{H}$ & -3.58510 & 7.95820 & 15.69120 \\
\hline $\mathrm{O}$ & -1.10880 & 0.35780 & 2.43010 & $\mathrm{H}$ & -2.47840 & -6.33390 & 11.35530 \\
\hline C & -1.52230 & 2.53210 & 3.92100 & $\mathrm{H}$ & -4.17380 & -6.66300 & 11.73710 \\
\hline $\mathrm{H}$ & -6.42130 & 3.15680 & 3.13460 & $\mathrm{H}$ & -3.74720 & -5.93540 & 10.15520 \\
\hline $\mathrm{H}$ & -1.93680 & 3.38510 & 4.45170 & C & 3.45090 & 3.29000 & -2.79740 \\
\hline $\mathrm{H}$ & -1.08600 & 1.81640 & 4.62070 & C & 2.25830 & 3.74450 & -3.42410 \\
\hline $\mathrm{H}$ & -0.73900 & 2.85250 & 3.23090 & C & 3.49500 & 5.65080 & -4.41260 \\
\hline $\mathrm{H}$ & -2.05870 & -1.37140 & 0.91290 & C & 2.33040 & 4.83990 & -4.37410 \\
\hline C & -8.68460 & 2.16100 & 1.90580 & C & 1.31260 & 5.11520 & -5.33680 \\
\hline C & -9.59140 & 1.71910 & 0.90840 & C & 1.32380 & 6.25820 & -6.09550 \\
\hline C & -10.70480 & 2.53990 & 0.56950 & $\mathrm{H}$ & 0.50130 & 4.42210 & -5.50680 \\
\hline C & -10.88580 & 3.74700 & 1.17810 & C & 0.19990 & 6.51670 & -7.03730 \\
\hline $\mathrm{H}$ & -11.34380 & 2.25550 & -0.25740 & C & 2.39160 & 7.19220 & -5.98220 \\
\hline $\mathrm{H}$ & -11.66560 & 4.42440 & 0.84210 & C & 3.51490 & 6.86110 & -5.17720 \\
\hline C & -9.10950 & 3.22580 & 2.79860 & C & 4.65080 & 7.72530 & -5.16840 \\
\hline C & -10.10190 & 4.11780 & 2.30450 & $\mathrm{C}$ & 4.56900 & 8.93510 & -5.87340 \\
\hline
\end{tabular}



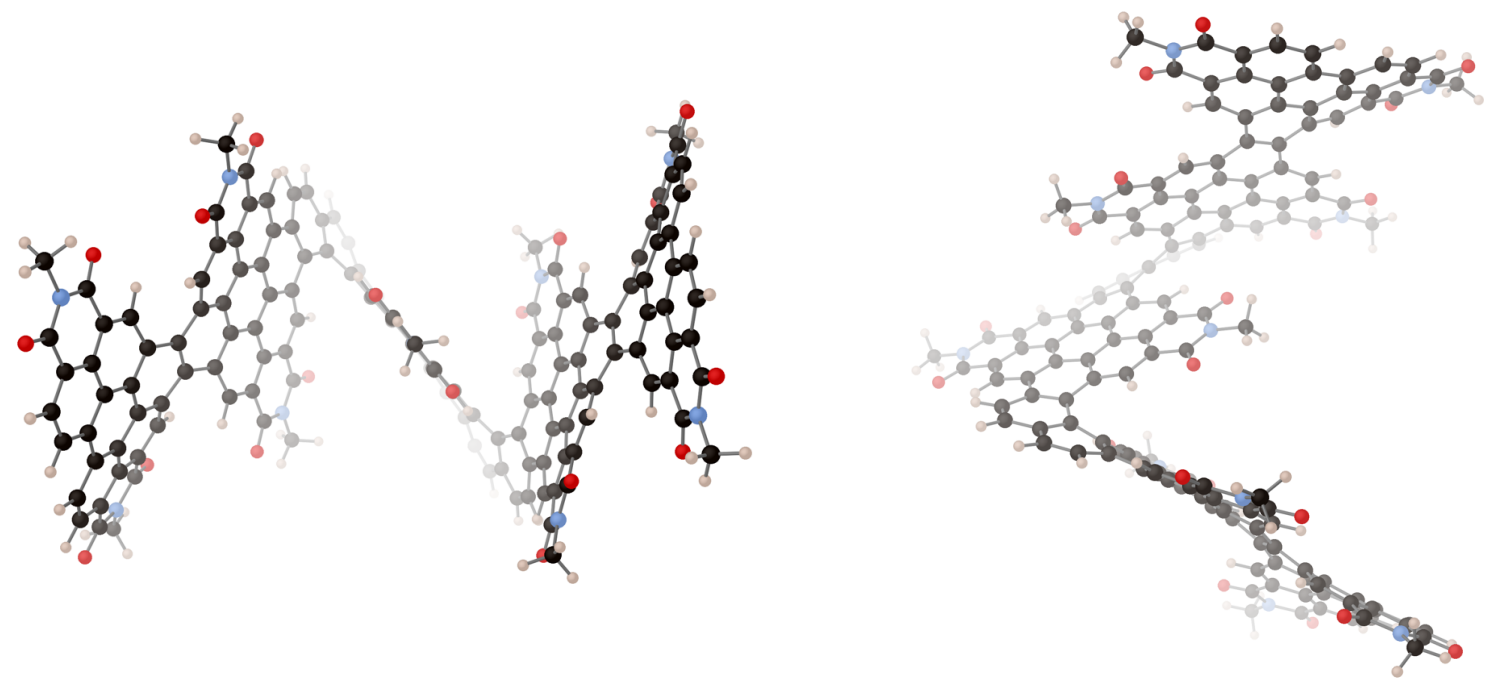

Figure S35. DFT-optimized (B3LYP/6-31G**) structure of $M$ P-M[6][6]-MP from different perspectives (total energy $=-7958.7683$ hartrees).

Cartesian Coordinates of $\boldsymbol{M P - M}$ [6][6]-MP (from left to right: atom; $x, y, z$ in $\AA$ )

\begin{tabular}{|c|c|c|c|c|c|c|c|c|c|c|c|}
\hline C & 0.64240 & 1.46560 & -2.07410 & $\mathrm{C}$ & -11.03900 & 4.97790 & 2.53320 & C & 3.13260 & 9.73580 & -4.23070 \\
\hline C & -0.52110 & 0.63880 & -2.18440 & $\mathrm{C}$ & -10.23860 & 5.49060 & 3.50960 & C & 2.17450 & 8.84970 & -4.71050 \\
\hline C & -1.69120 & 1.16380 & -2.77680 & $\mathrm{H}$ & -11.84260 & 5.56930 & 2.10650 & $\mathrm{H}$ & 4.87920 & 10.00550 & -3.04020 \\
\hline C & -1.71660 & 2.52080 & -3.16890 & $\mathrm{H}$ & -10.38990 & 6.50740 & 3.84830 & $\mathrm{H}$ & 3.10790 & 10.76310 & -4.57750 \\
\hline C & -0.59390 & 3.31110 & -3.04830 & $\mathrm{C}$ & -9.31670 & 4.64630 & 4.19240 & C & 1.19030 & 9.30850 & -5.72150 \\
\hline C & 0.64760 & 2.79720 & -2.58410 & $\mathrm{C}$ & -9.16110 & 3.29500 & 3.78690 & C & 4.21010 & 5.20320 & -2.12990 \\
\hline C & -0.54570 & -0.68910 & -1.65860 & $\mathrm{C}$ & -8.07540 & 2.83230 & 5.97980 & C & 4.22130 & 3.83940 & -1.75630 \\
\hline C & -1.72680 & -1.47850 & -1.72300 & $\mathrm{C}$ & -8.60210 & 2.35150 & 4.74910 & C & 5.47620 & 3.27150 & -1.38920 \\
\hline C & -2.84470 & -0.93910 & -2.40850 & C & -8.66750 & 0.94490 & 4.57120 & C & 6.57660 & 4.05130 & -1.14460 \\
\hline C & -2.84100 & 0.34690 & -2.90360 & $\mathrm{C}$ & -8.13430 & 0.06810 & 5.48960 & $\mathrm{H}$ & 5.60820 & 2.20230 & -1.31790 \\
\hline C & 1.75700 & 0.98640 & -1.32300 & $\mathrm{H}$ & -9.14860 & 0.51420 & 3.70820 & C & 7.83880 & 3.39460 & -0.71730 \\
\hline C & 1.71620 & -0.32200 & -0.76140 & $\mathrm{C}$ & -7.56690 & 1.92910 & 6.96060 & C & 6.50870 & 5.46360 & -1.29660 \\
\hline C & 0.61570 & -1.18950 & -0.99870 & $\mathrm{C}$ & -7.55390 & 0.54370 & 6.68630 & C & 5.33960 & 6.03970 & -1.86090 \\
\hline C & 2.86020 & 1.83810 & -1.06220 & $\mathrm{C}$ & -6.93690 & -0.34260 & 7.59810 & C & 5.31930 & 7.43370 & -2.15730 \\
\hline C & 3.76110 & 1.44100 & -0.03780 & $\mathrm{C}$ & -6.45140 & 0.12000 & 8.80110 & C & 6.42140 & 8.21260 & -1.76890 \\
\hline C & 3.74540 & 0.16940 & 0.48850 & $\mathrm{C}$ & -6.60700 & 1.47570 & 9.19220 & C & 7.54570 & 7.64140 & -1.17400 \\
\hline C & 2.76820 & -0.76210 & 0.07290 & $\mathrm{C}$ & -7.06730 & 2.39490 & 8.21550 & C & 7.61240 & 6.26810 & -0.96410 \\
\hline C & 2.78290 & -2.09280 & 0.54950 & $\mathrm{H}$ & -5.94180 & -0.59290 & 9.43190 & $\mathrm{H}$ & 6.42420 & 9.27920 & -1.95360 \\
\hline C & 1.77930 & -2.96860 & 0.19720 & $\mathrm{C}$ & -8.60940 & 5.14910 & 5.35370 & $\mathrm{H}$ & 8.39940 & 8.24940 & -0.89470 \\
\hline C & 0.66200 & -2.53070 & -0.55680 & $\mathrm{C}$ & -8.02390 & 4.23120 & 6.25480 & C & 8.84890 & 5.65590 & -0.41810 \\
\hline C & -4.08110 & 0.88450 & -3.52530 & $\mathrm{C}$ & -7.73160 & 6.99710 & 6.67340 & $\mathrm{~N}$ & 0.32940 & 8.32710 & -6.24380 \\
\hline $\mathrm{N}$ & -4.05970 & 2.24670 & -3.86950 & $\mathrm{C}$ & -8.45980 & 6.53670 & 5.59790 & $\mathrm{~N}$ & 8.88230 & 4.25560 & -0.33990 \\
\hline C & -2.97320 & 3.11870 & -3.69110 & $\mathrm{H}$ & -8.84810 & 7.27670 & 4.91100 & $\mathrm{O}$ & 1.14010 & 10.46940 & -6.10490 \\
\hline C & 4.75070 & -0.20280 & 1.51630 & C & -7.19410 & 6.08670 & 7.61450 & $\mathrm{O}$ & -0.45830 & 6.19580 & -6.40600 \\
\hline$N$ & 4.75920 & -1.55380 & 1.90970 & $\mathrm{C}$ & -7.38760 & 4.69860 & 7.44200 & $\mathrm{O}$ & 7.96510 & 2.17910 & -0.67400 \\
\hline C & 3.85540 & -2.53450 & 1.47730 & $\mathrm{C}$ & -6.94440 & 3.79530 & 8.45180 & $\mathrm{O}$ & 9.81640 & 6.31640 & -0.06450 \\
\hline $\mathrm{O}$ & -5.07790 & 0.19650 & -3.70300 & C & -6.31480 & 4.27550 & 9.63600 & C & -0.62300 & 8.71270 & -7.29020 \\
\hline $\mathrm{O}$ & -3.08280 & 4.30300 & -3.97340 & $\mathrm{C}$ & -5.94140 & 5.64750 & 9.64570 & C & 10.12480 & 3.66620 & 0.16890 \\
\hline $\mathrm{O}$ & 3.95190 & -3.68520 & 1.88240 & $\mathrm{C}$ & -6.40010 & 6.53380 & 8.69350 & $\mathrm{H}$ & -0.49810 & 9.77630 & -7.47780 \\
\hline $\mathrm{O}$ & 5.53700 & 0.60500 & 1.98840 & $\mathrm{H}$ & -5.27350 & 6.04880 & 10.39220 & $\mathrm{H}$ & -0.42840 & 8.13640 & -8.19740 \\
\hline C & -5.27070 & 2.84270 & -4.44290 & C & -6.04880 & 7.97360 & 8.80110 & $\mathrm{H}$ & -1.64120 & 8.49610 & -6.96040 \\
\hline C & 5.77000 & -1.99520 & 2.87560 & $\mathrm{~N}$ & -6.65140 & 8.84200 & 7.87590 & $\mathrm{H}$ & 10.95950 & 3.93510 & -0.48220 \\
\hline C & -1.72880 & -2.81820 & -1.14610 & $\mathrm{C}$ & -7.49580 & 8.45650 & 6.82160 & $\mathrm{H}$ & 10.33150 & 4.05340 & 1.16860 \\
\hline C & -0.48290 & -3.38730 & -0.77780 & C & -6.82290 & -1.78870 & 7.27910 & $\mathrm{H}$ & 9.99340 & 2.58730 & 0.19370 \\
\hline C & -0.36200 & -4.79780 & -0.62370 & $\mathrm{~N}$ & -7.50050 & -2.22030 & 6.12280 & & & & \\
\hline C & -1.43040 & -5.60690 & -0.86920 & $\mathrm{C}$ & -8.16500 & -1.38950 & 5.20970 & & & & \\
\hline C & -2.72610 & -5.05380 & -1.05290 & $\mathrm{O}$ & -5.29120 & 8.41020 & 9.65420 & & & & \\
\hline C & -2.91610 & -3.64230 & -0.99910 & $\mathrm{O}$ & -7.98920 & 9.27910 & 6.06210 & & & & \\
\hline C & -3.83620 & -5.91480 & -1.25970 & $\mathrm{O}$ & -6.20390 & -2.57150 & 7.98490 & & & & \\
\hline C & -5.09370 & -5.40210 & -1.37330 & $\mathrm{O}$ & -8.71510 & -1.86670 & 4.22630 & & & & \\
\hline
\end{tabular}




\begin{tabular}{|c|c|c|c|c|c|c|c|}
\hline C & -5.34530 & -4.03030 & -1.08310 & $\mathrm{C}$ & -6.34350 & 10.26680 & 8.03920 \\
\hline C & -4.26680 & -3.16780 & -0.75130 & $\mathrm{C}$ & -7.51530 & -3.65350 & 5.81540 \\
\hline C & -6.70750 & -3.53520 & -1.08440 & $\mathrm{H}$ & -6.87580 & 10.81210 & 7.26370 \\
\hline C & -6.98540 & -2.27510 & -0.50730 & $\mathrm{H}$ & -6.65840 & 10.60140 & 9.02990 \\
\hline C & -5.92920 & -1.50090 & 0.06820 & $\mathrm{H}$ & -5.26680 & 10.42500 & 7.95050 \\
\hline C & -4.57680 & -1.92820 & -0.04350 & $\mathrm{H}$ & -7.03230 & -3.83480 & 4.85260 \\
\hline C & -7.76980 & -4.27050 & -1.66910 & $\mathrm{H}$ & -6.98390 & -4.16960 & 6.61110 \\
\hline C & -9.05580 & -3.77660 & -1.69700 & $\mathrm{H}$ & -8.54800 & -4.00290 & 5.75070 \\
\hline C & -9.35410 & -2.52920 & -1.10010 & C & -6.08860 & 3.35140 & 10.74270 \\
\hline C & -8.32350 & -1.78740 & -0.47390 & C & -6.27950 & 1.96120 & 10.52210 \\
\hline C & -10.67220 & -2.01300 & -1.12530 & C & -5.69250 & 3.79480 & 12.06980 \\
\hline C & -10.95360 & -0.79760 & -0.54170 & C & -5.23200 & 2.83890 & 13.01760 \\
\hline C & -9.95510 & -0.05110 & 0.13190 & C & -5.21230 & 5.56270 & 13.69410 \\
\hline C & -8.63820 & -0.56480 & 0.18260 & C & -5.78210 & 5.14650 & 12.51750 \\
\hline C & -10.12990 & -4.56320 & -2.35770 & C & -5.32160 & 6.99100 & 14.08480 \\
\hline $\mathrm{N}$ & -11.41810 & -4.00810 & -2.34940 & $\mathrm{H}$ & -6.33380 & 5.88840 & 11.96090 \\
\hline C & -11.77010 & -2.77270 & -1.78050 & C & -4.54710 & 4.63860 & 14.54670 \\
\hline O & -12.91900 & -2.35370 & -1.82760 & C & -4.62590 & 3.25320 & 14.24630 \\
\hline O & -9.93080 & -5.64650 & -2.89090 & C & -4.12470 & 2.30550 & 15.18880 \\
\hline C & -12.46560 & -4.80220 & -3.00000 & C & -3.43990 & 2.79020 & 16.31380 \\
\hline $\mathrm{H}$ & -0.71970 & 4.35390 & -3.29170 & C & -3.31660 & 4.15480 & 16.57070 \\
\hline $\mathrm{H}$ & -3.74270 & -1.52290 & -2.54910 & C & -3.90200 & 5.08260 & 15.71500 \\
\hline $\mathrm{H}$ & 4.48010 & 2.12950 & 0.37830 & $\mathrm{H}$ & -3.01600 & 2.09550 & 17.02770 \\
\hline $\mathrm{H}$ & 1.83480 & -3.97350 & 0.59390 & $\mathrm{H}$ & -2.80560 & 4.51480 & 17.45660 \\
\hline $\mathrm{H}$ & -5.04770 & 3.25580 & -5.42910 & C & -3.87730 & 6.52360 & 16.06480 \\
\hline $\mathrm{H}$ & -6.02210 & 2.06050 & -4.51760 & C & -5.49090 & 1.45230 & 12.80910 \\
\hline $\mathrm{H}$ & -5.62240 & 3.65530 & -3.80370 & C & -6.15970 & 1.03770 & 11.63300 \\
\hline $\mathrm{H}$ & 6.40690 & -1.14380 & 3.10200 & C & -6.76160 & -0.25520 & 11.63750 \\
\hline $\mathrm{H}$ & 5.28320 & -2.35980 & 3.78310 & C & -6.53900 & -1.15340 & 12.64900 \\
\hline $\mathrm{H}$ & 6.35340 & -2.81300 & 2.44810 & $\mathrm{H}$ & -7.45970 & -0.54800 & 10.86680 \\
\hline $\mathrm{H}$ & 0.60400 & -5.23280 & -0.40060 & C & -7.23770 & -2.46520 & 12.61460 \\
\hline $\mathrm{H}$ & -1.31950 & -6.68730 & -0.87980 & C & -5.67880 & -0.81880 & 13.73260 \\
\hline $\mathrm{H}$ & -3.66090 & -6.98270 & -1.34930 & C & -5.18550 & 0.50950 & 13.84140 \\
\hline $\mathrm{H}$ & -5.92180 & -6.06710 & -1.57970 & C & -4.43320 & 0.88870 & 14.99220 \\
\hline $\mathrm{H}$ & -7.60080 & -5.22160 & -2.15540 & C & -4.10850 & -0.10580 & 15.92930 \\
\hline $\mathrm{H}$ & -11.98160 & -0.46170 & -0.58470 & C & -4.57680 & -1.41270 & 15.79900 \\
\hline $\mathrm{H}$ & -12.22810 & -4.93620 & -4.05770 & C & -5.39090 & -1.76840 & 14.72880 \\
\hline $\mathrm{H}$ & -12.52300 & -5.78800 & -2.53450 & $\mathrm{H}$ & -3.51470 & 0.14220 & 16.79970 \\
\hline $\mathrm{H}$ & -13.40550 & -4.26780 & -2.88630 & $\mathrm{H}$ & -4.35100 & -2.16380 & 16.54810 \\
\hline C & -6.25940 & -0.30270 & 0.77620 & C & -5.99130 & -3.12390 & 14.67180 \\
\hline C & -7.60980 & 0.15590 & 0.86610 & $\mathrm{~N}$ & -4.61010 & 7.38310 & 15.22720 \\
\hline C & -7.92640 & 1.32170 & 1.61350 & $\mathrm{~N}$ & -6.90520 & -3.36920 & 13.63610 \\
\hline C & -6.85710 & 2.06520 & 2.17350 & $\mathrm{O}$ & -3.27780 & 6.94570 & 17.04380 \\
\hline C & -5.55030 & 1.64050 & 2.08250 & $\mathrm{O}$ & -5.97650 & 7.80880 & 13.45460 \\
\hline C & -5.23240 & 0.44320 & 1.39990 & $\mathrm{O}$ & -5.74570 & -3.99450 & 15.49590 \\
\hline C & -3.59150 & -1.17780 & 0.64890 & $\mathrm{O}$ & -8.05720 & -2.74890 & 11.75210 \\
\hline C & -3.89960 & -0.02520 & 1.33770 & C & -4.67620 & 8.80840 & 15.56660 \\
\hline C & -2.81110 & 0.73420 & 2.00320 & C & -7.55490 & -4.68370 & 13.64520 \\
\hline $\mathrm{N}$ & -3.17710 & 1.92610 & 2.64660 & $\mathrm{H}$ & -4.04650 & 8.97240 & 16.43760 \\
\hline C & -4.48130 & 2.44690 & 2.72790 & $\mathrm{H}$ & -4.32900 & 9.40350 & 14.71980 \\
\hline O & -4.71100 & 3.49880 & 3.31040 & $\mathrm{H}$ & -5.70910 & 9.09150 & 15.78200 \\
\hline O & -1.64020 & 0.37510 & 1.99390 & $\mathrm{H}$ & -6.81290 & -5.46930 & 13.48620 \\
\hline C & -2.08790 & 2.67010 & 3.28490 & $\mathrm{H}$ & -8.02920 & -4.84990 & 14.61430 \\
\hline $\mathrm{H}$ & -7.03840 & 3.00230 & 2.67710 & $\mathrm{H}$ & -8.29280 & -4.69390 & 12.84690 \\
\hline $\mathrm{H}$ & -2.51450 & 3.56470 & 3.73180 & C & 2.98550 & 3.08320 & -1.79740 \\
\hline $\mathrm{H}$ & -1.61170 & 2.04910 & 4.04690 & C & 1.88570 & 3.57410 & -2.55000 \\
\hline $\mathrm{H}$ & -1.33400 & 2.93470 & 2.54040 & C & 3.10510 & 5.71610 & -2.87010 \\
\hline $\mathrm{H}$ & -2.56450 & -1.50390 & 0.67880 & C & 2.03030 & 4.85500 & -3.22820 \\
\hline C & -9.30740 & 1.77870 & 1.68350 & $\mathrm{C}$ & 1.17530 & 5.30170 & -4.27860 \\
\hline C & -10.25170 & 1.20280 & 0.79620 & C & 1.23090 & 6.58270 & -4.76820 \\
\hline C & -11.46650 & 1.89370 & 0.51710 & $\mathrm{H}$ & 0.47630 & 4.63270 & -4.75690 \\
\hline C & -11.68090 & 3.13730 & 1.03180 & C & 0.30540 & 6.97910 & -5.85950 \\
\hline $\mathrm{H}$ & -12.17120 & 1.48380 & -0.19340 & C & 2.17300 & 7.51770 & -4.25670 \\
\hline $\mathrm{H}$ & -12.53660 & 3.72770 & 0.71710 & C & 3.15970 & 7.06660 & -3.34010 \\
\hline C & -9.72970 & 2.89380 & 2.51010 & C & 4.19360 & 7.96210 & -2.92760 \\
\hline C & -10.81650 & 3.66960 & 2.02610 & $C$ & 4.12590 & 9.29600 & -3.35760 \\
\hline
\end{tabular}


a. $\mathrm{WH}[6]$
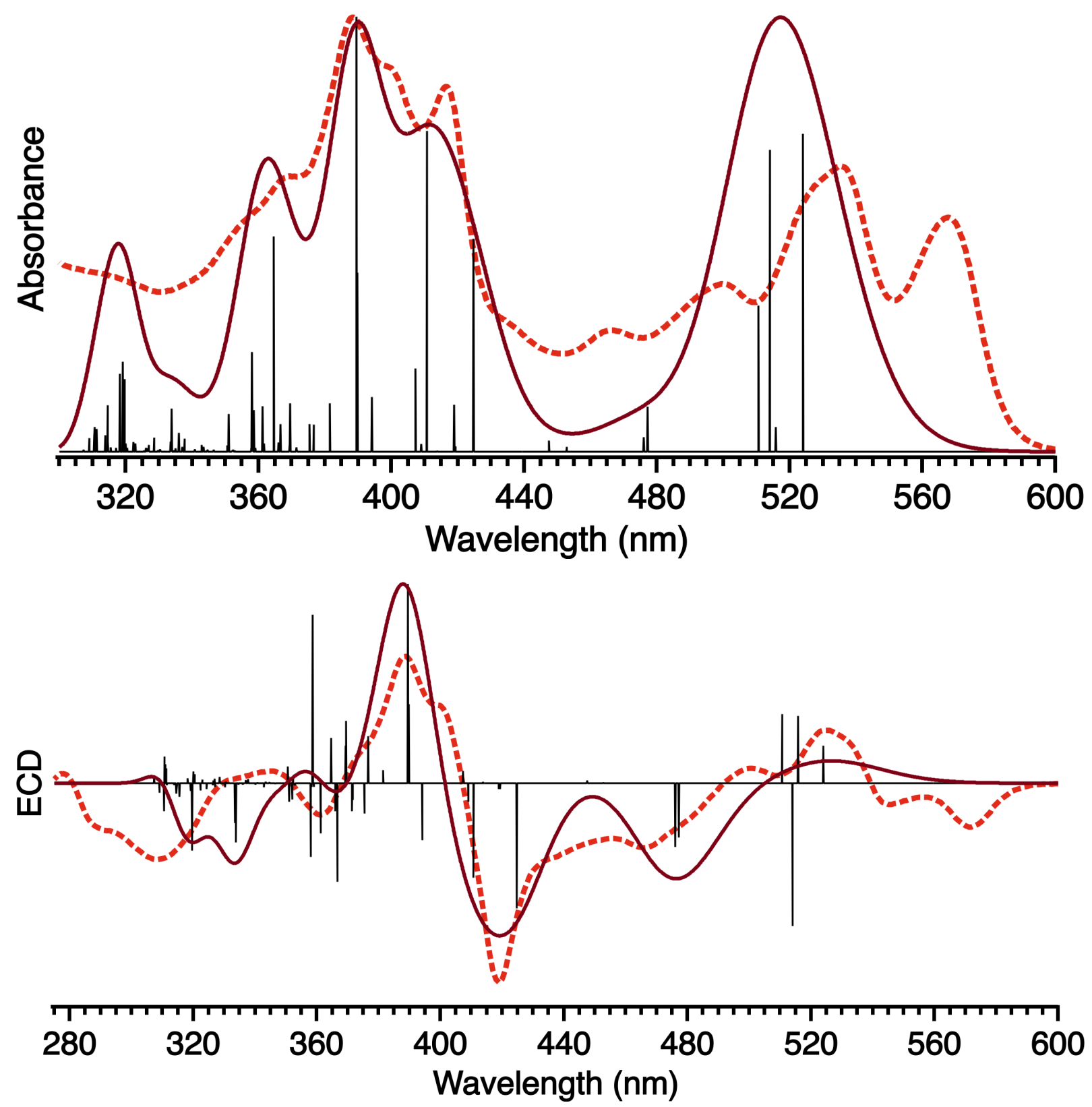

Figure S36. TDDFT-predicted (CAM-B3LYP/6-31++G**) UV-visible absorbance and ECD spectra of MM-M[6]-MM (zero bandwidth in black, $\sigma=0.13 \mathrm{eV}$ in red) overlaid on the experimental spectra of WH[6] in dichloromethane $\left(10^{-6} \mathrm{M}, 1 \mathrm{~cm}\right.$ path length) at room temperature (dotted orange). A positive wavelength shift of $56 \mathrm{~nm}$ has been applied to the calculated spectra. The intensities of the bandbroadened calculated spectra have also been scaled by the following factors: UV-visible absorbance, $0.7215 ; \mathrm{ECD}, 0.3171$. 
Table S3. TDDFT-Calculated Singlet Excited State Data for MM-M[6]-MM†

\begin{tabular}{|c|c|c|c|c|c|}
\hline Excited State & $E(e V)$ & $\lambda(\mathrm{nm})$ & $\lambda+56(\mathrm{~nm})$ & f & $R^{r}\left(10^{-40}\right.$ cgs $)$ \\
\hline 1 & 2.6493 & 467.98 & 523.98 & 0.8862 & 356.338 \\
\hline 2 & 2.6963 & 459.82 & 515.82 & 0.0688 & 624.3125 \\
\hline 3 & 2.7074 & 457.95 & 513.95 & 0.8414 & -1321.1 \\
\hline 4 & 2.7271 & 454.64 & 510.64 & 0.4065 & 633.947 \\
\hline 5 & 2.9438 & 421.17 & 477.17 & 0.1255 & -462.7298 \\
\hline 6 & 2.9523 & 419.96 & 475.96 & 0.0402 & -539.8456 \\
\hline 7 & 3.1243 & 396.84 & 452.84 & 0.013 & 2.879 \\
\hline 8 & 3.1672 & 391.46 & 447.46 & 0.0311 & 21.5107 \\
\hline 9 & 3.3623 & 368.74 & 424.74 & 0.5931 & -932.474 \\
\hline 10 & 3.4124 & 363.33 & 419.33 & 0.0147 & -41.5493 \\
\hline 11 & 3.4165 & 362.9 & 418.9 & 0.131 & -43.9439 \\
\hline 12 & 3.465 & 357.82 & 413.82 & 0.0008 & 9.4295 \\
\hline 13 & 3.4957 & 354.68 & 410.68 & 0.8933 & -675.6892 \\
\hline 14 & 3.5124 & 352.99 & 408.99 & 0.0219 & -131.7004 \\
\hline 15 & 3.529 & 351.33 & 407.33 & 0.2313 & 86.6241 \\
\hline 16 & 3.6668 & 338.13 & 394.13 & 0.1528 & -387.8887 \\
\hline 17 & 3.7148 & 333.76 & 389.76 & 0.4988 & 534.5187 \\
\hline 18 & 3.7181 & 333.46 & 389.46 & 1.2125 & 1343.1625 \\
\hline 19 & 3.8092 & 325.49 & 381.49 & 0.1342 & 86.2574 \\
\hline 20 & 3.8668 & 320.64 & 376.64 & 0.0751 & 305.6531 \\
\hline 21 & 3.882 & 319.38 & 375.38 & 0.0765 & -194.5909 \\
\hline 22 & 3.9008 & 317.84 & 373.84 & 0.0002 & -1.7899 \\
\hline 23 & 3.9284 & 315.61 & 371.61 & 0.0037 & -105.4853 \\
\hline 24 & 3.9314 & 315.37 & 371.37 & 0.0128 & -178.7458 \\
\hline 25 & 3.9549 & 313.5 & 369.5 & 0.1347 & 395.5409 \\
\hline 26 & 3.957 & 313.33 & 369.33 & 0.019 & 238.6282 \\
\hline 27 & 3.9914 & 310.63 & 366.63 & 0.0771 & -618.422 \\
\hline 28 & 3.999 & 310.04 & 366.04 & 0.0256 & -174.9103 \\
\hline 29 & 4.0174 & 308.62 & 364.62 & 0.6001 & 280.8797 \\
\hline 30 & 4.0556 & 305.71 & 361.71 & 0.0232 & -4.0205 \\
\hline 31 & 4.0624 & 305.2 & 361.2 & 0.1274 & -308.742 \\
\hline 32 & 4.0917 & 303.01 & 359.01 & 0.0103 & -23.1126 \\
\hline 33 & 4.0976 & 302.58 & 358.58 & 0.0736 & 515.7932 \\
\hline 34 & 4.0979 & 302.55 & 358.55 & 0.0427 & 516.6841 \\
\hline 35 & 4.1009 & 302.34 & 358.34 & 0.0455 & -23.0877 \\
\hline 36 & 4.1039 & 302.12 & 358.12 & 0.0476 & -29.4874 \\
\hline 37 & 4.1056 & 301.98 & 357.98 & 0.2779 & -448.7637 \\
\hline 38 & 4.108 & 301.81 & 357.81 & 0.0119 & -0.0355 \\
\hline 39 & 4.1764 & 296.87 & 352.87 & 0.0017 & -9.6649 \\
\hline 40 & 4.1777 & 296.77 & 352.77 & 0.0029 & -7.397 \\
\hline 41 & 4.1813 & 296.52 & 352.52 & 0.0011 & -5.1692 \\
\hline 42 & 4.1833 & 296.38 & 352.38 & 0.0011 & 0.4974 \\
\hline 43 & 4.1848 & 296.28 & 352.28 & 0.005 & 29.4198 \\
\hline 44 & 4.1879 & 296.05 & 352.05 & 0.0033 & -98.4656 \\
\hline 45 & 4.2033 & 294.97 & 350.97 & 0.1052 & -111.3112 \\
\hline 46 & 4.2082 & 294.62 & 350.62 & 0.017 & 100.1849 \\
\hline 47 & 4.2686 & 290.46 & 346.46 & 0.0059 & -3.5998 \\
\hline 48 & 4.2944 & 288.71 & 344.71 & 0.0037 & 5.7926 \\
\hline 49 & 4.2965 & 288.57 & 344.57 & 0.0044 & 3.5895 \\
\hline 50 & 4.3022 & 288.19 & 344.19 & 0.0021 & 0.5054 \\
\hline 51 & 4.3146 & 287.36 & 343.36 & 0.0134 & 7.2455 \\
\hline 52 & 4.3212 & 286.92 & 342.92 & 0.0191 & -22.7149 \\
\hline 53 & 4.3538 & 284.77 & 340.77 & 0.0065 & 3.7846 \\
\hline 54 & 4.3634 & 284.14 & 340.14 & 0.0009 & -7.4391 \\
\hline
\end{tabular}




\begin{tabular}{|c|c|c|c|c|c|}
\hline 55 & 4.4017 & 281.68 & 337.68 & 0.0366 & 22.3076 \\
\hline 56 & 4.409 & 281.21 & 337.21 & 0.0003 & 0.3582 \\
\hline 57 & 4.412 & 281.02 & 337.02 & 0.0138 & 18.3276 \\
\hline 58 & 4.4201 & 280.5 & 336.5 & 0.0003 & -1.8906 \\
\hline 59 & 4.4272 & 280.05 & 336.05 & 0.0529 & -8.7994 \\
\hline 60 & 4.4433 & 279.03 & 335.03 & 0.0097 & -0.2962 \\
\hline 61 & 4.4503 & 278.6 & 334.6 & 0.0034 & -8.5903 \\
\hline 62 & 4.4627 & 277.82 & 333.82 & 0.1202 & -332.6644 \\
\hline 63 & 4.4679 & 277.5 & 333.5 & 0.0282 & -223.5508 \\
\hline 64 & 4.4998 & 275.53 & 331.53 & 0.002 & 2.5237 \\
\hline 65 & 4.5154 & 274.58 & 330.58 & 0.0008 & 8.3139 \\
\hline 66 & 4.5177 & 274.44 & 330.44 & 0.0011 & 7.1589 \\
\hline 67 & 4.5194 & 274.34 & 330.34 & 0.0069 & -22.3022 \\
\hline 68 & 4.5296 & 273.72 & 329.72 & 0.0014 & 0.0222 \\
\hline 69 & 4.5304 & 273.67 & 329.67 & 0.0033 & -1.3303 \\
\hline 70 & 4.55 & 272.49 & 328.49 & 0.0385 & 33.9714 \\
\hline 71 & 4.5552 & 272.18 & 328.18 & 0.0013 & 1.05 \\
\hline 72 & 4.5746 & 271.03 & 327.03 & 0.0043 & -20.7074 \\
\hline 73 & 4.5767 & 270.9 & 326.9 & 0.0189 & 23.6251 \\
\hline 74 & 4.5793 & 270.75 & 326.75 & 0.0044 & -7.2427 \\
\hline 75 & 4.5838 & 270.49 & 326.49 & 0.009 & 14.0711 \\
\hline 76 & 4.5873 & 270.28 & 326.28 & 0.0014 & -10.3736 \\
\hline 77 & 4.5896 & 270.14 & 326.14 & 0.0106 & -0.6027 \\
\hline 78 & 4.5998 & 269.54 & 325.54 & 0.0023 & -0.29 \\
\hline 79 & 4.6215 & 268.28 & 324.28 & 0.0014 & -32.0095 \\
\hline 80 & 4.6454 & 266.9 & 322.9 & 0.0227 & 20.4572 \\
\hline 81 & 4.6563 & 266.27 & 322.27 & 0.0275 & -39.0598 \\
\hline 82 & 4.6892 & 264.4 & 320.4 & 0.0107 & 48.3216 \\
\hline 83 & 4.6959 & 264.03 & 320.03 & 0.0225 & 63.5702 \\
\hline 84 & 4.7041 & 263.56 & 319.56 & 0.2017 & -359.4086 \\
\hline 85 & 4.7131 & 263.06 & 319.06 & 0.2501 & -39.7028 \\
\hline 86 & 4.7288 & 262.19 & 318.19 & 0.2169 & 25.4572 \\
\hline 87 & 4.7481 & 261.12 & 317.12 & 0.0112 & -1.0091 \\
\hline 88 & 4.777 & 259.54 & 315.54 & 0.0127 & -70.9066 \\
\hline 89 & 4.7942 & 258.62 & 314.62 & 0.0182 & -20.645 \\
\hline 90 & 4.7951 & 258.56 & 314.56 & 0.0096 & -1.8993 \\
\hline 91 & 4.7966 & 258.48 & 314.48 & 0.1299 & -54.7999 \\
\hline 92 & 4.8098 & 257.77 & 313.77 & 0.0461 & -9.7113 \\
\hline 93 & 4.834 & 256.48 & 312.48 & 0.0002 & -0.4836 \\
\hline 94 & 4.8546 & 255.39 & 311.39 & 0.0004 & -9.6976 \\
\hline 95 & 4.8587 & 255.18 & 311.18 & 0.0635 & 76.9326 \\
\hline 96 & 4.8641 & 254.9 & 310.9 & 0.0163 & 98.6522 \\
\hline 97 & 4.8695 & 254.61 & 310.61 & 0.0683 & 137.7009 \\
\hline 98 & 4.8708 & 254.54 & 310.54 & 0.0435 & -144.4195 \\
\hline 99 & 4.8997 & 253.04 & 309.04 & 0.0373 & -49.3421 \\
\hline 100 & 4.9338 & 251.29 & 307.29 & 0.0059 & 27.506 \\
\hline
\end{tabular}

tExcited state energies $(E)$, wavelengths $(\lambda)$, oscillator strengths $(f)$, and rotatory strengths in dipole length formalism $\left(R^{r}\right)$ were calculated at the CAM-B3LYP/6-31++ ${ }^{* *}$ level of theory. 

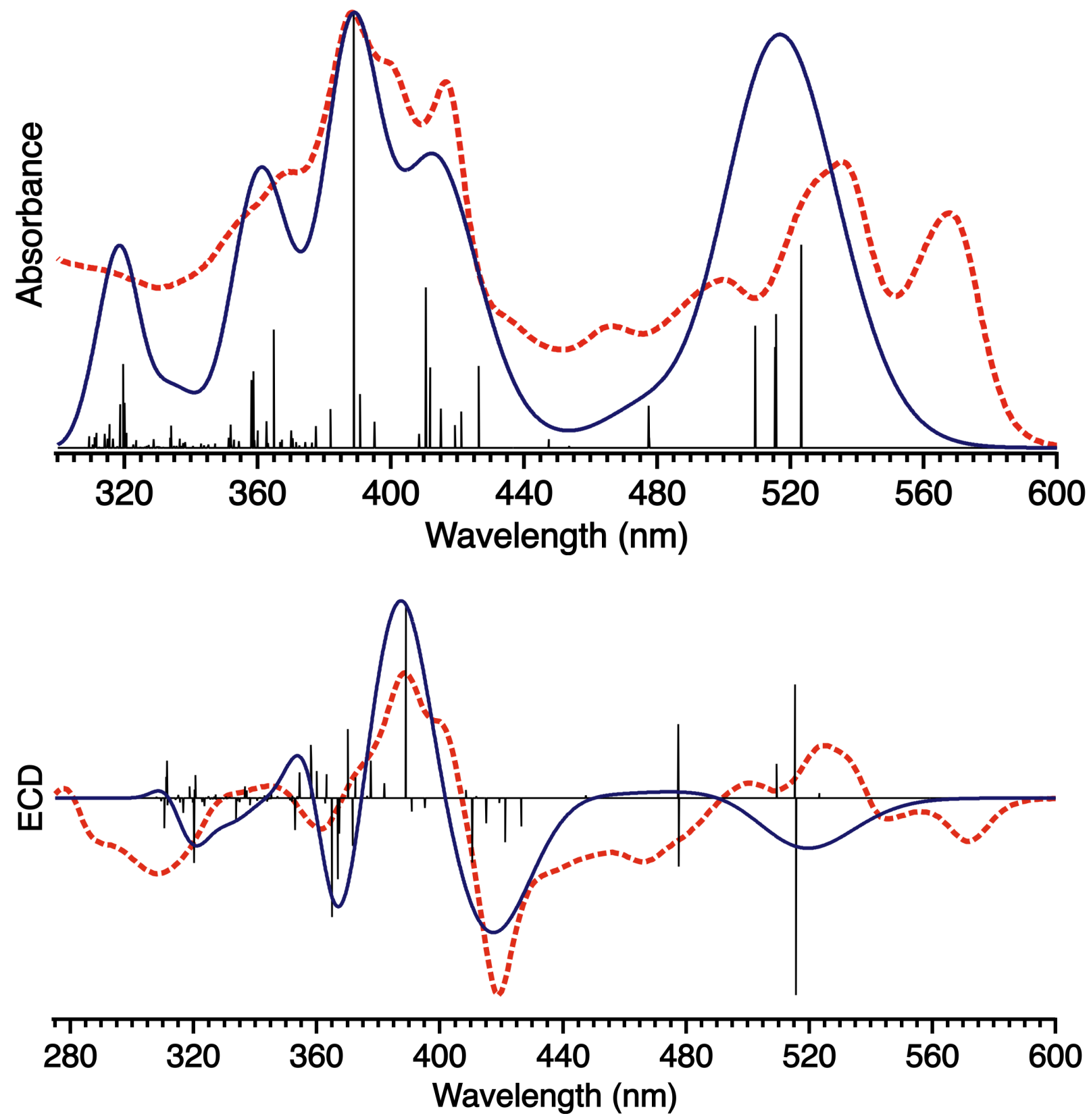

Figure S37. TDDFT-predicted (CAM-B3LYP/6-31++ $\mathrm{G}^{* *}$ ) UV-visible absorbance and ECD spectra of MM-M[6]-PP (zero bandwidth in black, $\sigma=0.13 \mathrm{eV}$ in blue) overlaid on the experimental spectra of WH[6] in dichloromethane $\left(10^{-6} \mathrm{M}, 1 \mathrm{~cm}\right.$ path length) at room temperature (dotted orange). A positive wavelength shift of $56 \mathrm{~nm}$ has been applied to the calculated spectra. The intensities of the bandbroadened calculated spectra have also been scaled by the following factors: UV-visible absorbance, $0.6975 ;$ ECD, 0.4754. 
Table S4. TDDFT-Calculated Singlet Excited State Data for MM-M[6]-PP†

\begin{tabular}{|c|c|c|c|c|c|}
\hline Excited State & $E(e V)$ & $\lambda(\mathrm{nm})$ & $\lambda+56(\mathrm{~nm})$ & f & $R^{r}\left(10^{-40} \mathrm{cgs}\right)$ \\
\hline 1 & 2.6539 & 467.17 & 523.17 & 0.7801 & 39.018 \\
\hline 2 & 2.6972 & 459.68 & 515.68 & 0.5135 & -1540.8837 \\
\hline 3 & 2.6995 & 459.28 & 515.28 & 0.3871 & 889.3915 \\
\hline 4 & 2.7348 & 453.35 & 509.35 & 0.4683 & 263.7604 \\
\hline 5 & 2.941 & 421.58 & 477.58 & 0.0393 & -493.2787 \\
\hline 6 & 2.9425 & 421.35 & 477.35 & 0.1624 & 529.591 \\
\hline 7 & 3.1194 & 397.47 & 453.47 & 0.0079 & 5.7719 \\
\hline 8 & 3.1677 & 391.4 & 447.4 & 0.033 & 16.6953 \\
\hline 9 & 3.3476 & 370.37 & 426.37 & 0.3155 & -180.3308 \\
\hline 10 & 3.3951 & 365.19 & 421.19 & 0.1393 & -273.5089 \\
\hline 11 & 3.4127 & 363.3 & 419.3 & 0.0869 & -29.5329 \\
\hline 12 & 3.4533 & 359.03 & 415.03 & 0.1503 & -154.4788 \\
\hline 13 & 3.4845 & 355.81 & 411.81 & 0.3096 & 9.8856 \\
\hline 14 & 3.4977 & 354.47 & 410.47 & 0.6161 & -389.0771 \\
\hline 15 & 3.5174 & 352.49 & 408.49 & 0.0539 & 48.3467 \\
\hline 16 & 3.6564 & 339.08 & 395.08 & 0.0998 & -56.2945 \\
\hline 17 & 3.7034 & 334.78 & 390.78 & 0.2071 & -78.1965 \\
\hline 18 & 3.7245 & 332.89 & 388.89 & 1.6695 & 1082.4508 \\
\hline 19 & 3.8042 & 325.91 & 381.91 & 0.1482 & 81.8282 \\
\hline 20 & 3.8568 & 321.47 & 377.47 & 0.0833 & 203.8006 \\
\hline 21 & 3.8703 & 320.34 & 376.34 & 0.0206 & 12.3711 \\
\hline 22 & 3.8954 & 318.28 & 374.28 & 0.023 & -52.1394 \\
\hline 23 & 3.9174 & 316.49 & 372.49 & 0.0099 & 112.4951 \\
\hline 24 & 3.9283 & 315.62 & 371.62 & 0.0217 & -256.3523 \\
\hline 25 & 3.9409 & 314.61 & 370.61 & 0.0365 & -1.7195 \\
\hline 26 & 3.9471 & 314.11 & 370.11 & 0.0662 & 368.4994 \\
\hline 27 & 3.9824 & 311.33 & 367.33 & 0.0318 & -189.9411 \\
\hline 28 & 3.9887 & 310.84 & 366.84 & 0.0217 & -430.4725 \\
\hline 29 & 4.0136 & 308.91 & 364.91 & 0.4541 & -626.5307 \\
\hline 30 & 4.0377 & 307.07 & 363.07 & 0.0181 & 125.8387 \\
\hline 31 & 4.0422 & 306.73 & 362.73 & 0.103 & -28.4754 \\
\hline 32 & 4.0788 & 303.97 & 359.97 & 0.0674 & 139.8281 \\
\hline 33 & 4.0908 & 303.08 & 359.08 & 0.0072 & -22.9188 \\
\hline 34 & 4.0911 & 303.06 & 359.06 & 0.0235 & 1.8924 \\
\hline 35 & 4.0939 & 302.85 & 358.85 & 0.2937 & -11.5486 \\
\hline 36 & 4.0989 & 302.48 & 358.48 & 0.0012 & 12.9518 \\
\hline 37 & 4.102 & 302.25 & 358.25 & 0.0722 & 195.0807 \\
\hline 38 & 4.1046 & 302.06 & 358.06 & 0.2606 & 273.8552 \\
\hline 39 & 4.1556 & 298.36 & 354.36 & 0.0259 & 129.9259 \\
\hline 40 & 4.1652 & 297.67 & 353.67 & 0.0003 & -4.1001 \\
\hline 41 & 4.1668 & 297.56 & 353.56 & 0.0014 & 9.8929 \\
\hline 42 & 4.1719 & 297.19 & 353.19 & 0.0003 & -2.7944 \\
\hline 43 & 4.1763 & 296.87 & 352.87 & 0.0314 & -162.0271 \\
\hline 44 & 4.1878 & 296.06 & 352.06 & 0.0124 & 10.375 \\
\hline 45 & 4.1906 & 295.86 & 351.86 & 0.0892 & -18.2367 \\
\hline 46 & 4.1981 & 295.33 & 351.33 & 0.0399 & -11.4757 \\
\hline 47 & 4.2584 & 291.15 & 347.15 & 0.016 & 9.5796 \\
\hline 48 & 4.2878 & 289.16 & 345.16 & 0.0131 & 32.141 \\
\hline 49 & 4.2908 & 288.95 & 344.95 & 0.0065 & -4.5557 \\
\hline 50 & 4.301 & 288.27 & 344.27 & 0.0008 & -7.0363 \\
\hline 51 & 4.3066 & 287.89 & 343.89 & 0.0085 & -18.513 \\
\hline 52 & 4.3201 & 287 & 343 & 0.0168 & 12.6134 \\
\hline 53 & 4.3434 & 285.46 & 341.46 & 0.0004 & 2.2833 \\
\hline 54 & 4.3563 & 284.61 & 340.61 & 0.0076 & -12.4876 \\
\hline
\end{tabular}




\begin{tabular}{|c|c|c|c|c|c|}
\hline 55 & 4.3919 & 282.3 & 338.3 & 0.0224 & -35.3431 \\
\hline 56 & 4.3956 & 282.06 & 338.06 & 0.0081 & 3.0661 \\
\hline 57 & 4.4029 & 281.6 & 337.6 & 0.019 & -5.3056 \\
\hline 58 & 4.4083 & 281.25 & 337.25 & 0.0069 & 36.616 \\
\hline 59 & 4.4181 & 280.63 & 336.63 & 0.0361 & 56.1811 \\
\hline 60 & 4.4334 & 279.66 & 335.66 & 0.0079 & -1.5401 \\
\hline 61 & 4.4461 & 278.86 & 334.86 & 0.0066 & -19.5951 \\
\hline 62 & 4.4576 & 278.14 & 334.14 & 0.0855 & -14.4584 \\
\hline 63 & 4.4636 & 277.77 & 333.77 & 0.0398 & -104.7298 \\
\hline 64 & 4.4974 & 275.68 & 331.68 & 0.0018 & 4.9498 \\
\hline 65 & 4.5103 & 274.89 & 330.89 & 0.0006 & 0.7616 \\
\hline 66 & 4.5119 & 274.79 & 330.79 & 0.001 & -4.4064 \\
\hline 67 & 4.5165 & 274.51 & 330.51 & 0.0055 & 5.9002 \\
\hline 68 & 4.5249 & 274 & 330 & 0.006 & -2.0763 \\
\hline 69 & 4.5287 & 273.77 & 329.77 & 0.0021 & -2.6923 \\
\hline 70 & 4.5295 & 273.72 & 329.72 & 0.0059 & -4.6625 \\
\hline 71 & 4.5455 & 272.76 & 328.76 & 0.0331 & -3.2995 \\
\hline 72 & 4.5571 & 272.07 & 328.07 & 0.0017 & -1.0593 \\
\hline 73 & 4.5704 & 271.28 & 327.28 & 0.0116 & -5.2289 \\
\hline 74 & 4.5738 & 271.07 & 327.07 & 0.0014 & 16.343 \\
\hline 75 & 4.5756 & 270.97 & 326.97 & 0.0001 & -3.7792 \\
\hline 76 & 4.5796 & 270.73 & 326.73 & 0.0083 & 5.9229 \\
\hline 77 & 4.5881 & 270.23 & 326.23 & 0.0043 & -6.0571 \\
\hline 78 & 4.6027 & 269.37 & 325.37 & 0.0023 & -6.0659 \\
\hline 79 & 4.6126 & 268.79 & 324.79 & 0.0043 & 8.3155 \\
\hline 80 & 4.6357 & 267.46 & 323.46 & 0.0304 & -37.3549 \\
\hline 81 & 4.6485 & 266.72 & 322.72 & 0.0125 & -19.0895 \\
\hline 82 & 4.6848 & 264.65 & 320.65 & 0.0572 & 103.7329 \\
\hline 83 & 4.6916 & 264.27 & 320.27 & 0.0072 & 38.9304 \\
\hline 84 & 4.6939 & 264.14 & 320.14 & 0.1732 & -292.2695 \\
\hline 85 & 4.702 & 263.68 & 319.68 & 0.3225 & -19.8226 \\
\hline 86 & 4.72 & 262.68 & 318.68 & 0.1673 & 52.453 \\
\hline 87 & 4.7348 & 261.86 & 317.86 & 0.0052 & -0.8262 \\
\hline 88 & 4.758 & 260.58 & 316.58 & 0.0355 & -66.7568 \\
\hline 89 & 4.778 & 259.49 & 315.49 & 0.0904 & -19.9576 \\
\hline 90 & 4.7866 & 259.02 & 315.02 & 0.0347 & 13.9062 \\
\hline 91 & 4.7933 & 258.66 & 314.66 & 0.0022 & 0.7355 \\
\hline 92 & 4.8045 & 258.06 & 314.06 & 0.0543 & -8.8592 \\
\hline 93 & 4.8353 & 256.42 & 312.42 & 0.0031 & 2.2798 \\
\hline 94 & 4.8497 & 255.65 & 311.65 & 0.0576 & -3.1896 \\
\hline 95 & 4.8534 & 255.46 & 311.46 & 0.0095 & -32.1529 \\
\hline 96 & 4.8569 & 255.27 & 311.27 & 0.0369 & 162.1311 \\
\hline 97 & 4.8608 & 255.07 & 311.07 & 0.0413 & 91.6125 \\
\hline 98 & 4.8724 & 254.46 & 310.46 & 0.0123 & -131.0643 \\
\hline 99 & 4.8923 & 253.42 & 309.42 & 0.0449 & -14.5173 \\
\hline 100 & 4.9221 & 251.9 & 307.9 & 0.0017 & 5.7317 \\
\hline
\end{tabular}

tExcited state energies $(E)$, wavelengths $(\lambda)$, oscillator strengths (f), and rotatory strengths in dipole length formalism ( $\left.R^{r}\right)$ were calculated at the CAM-B3LYP/6-31++G ${ }^{\star *}$ level of theory. 

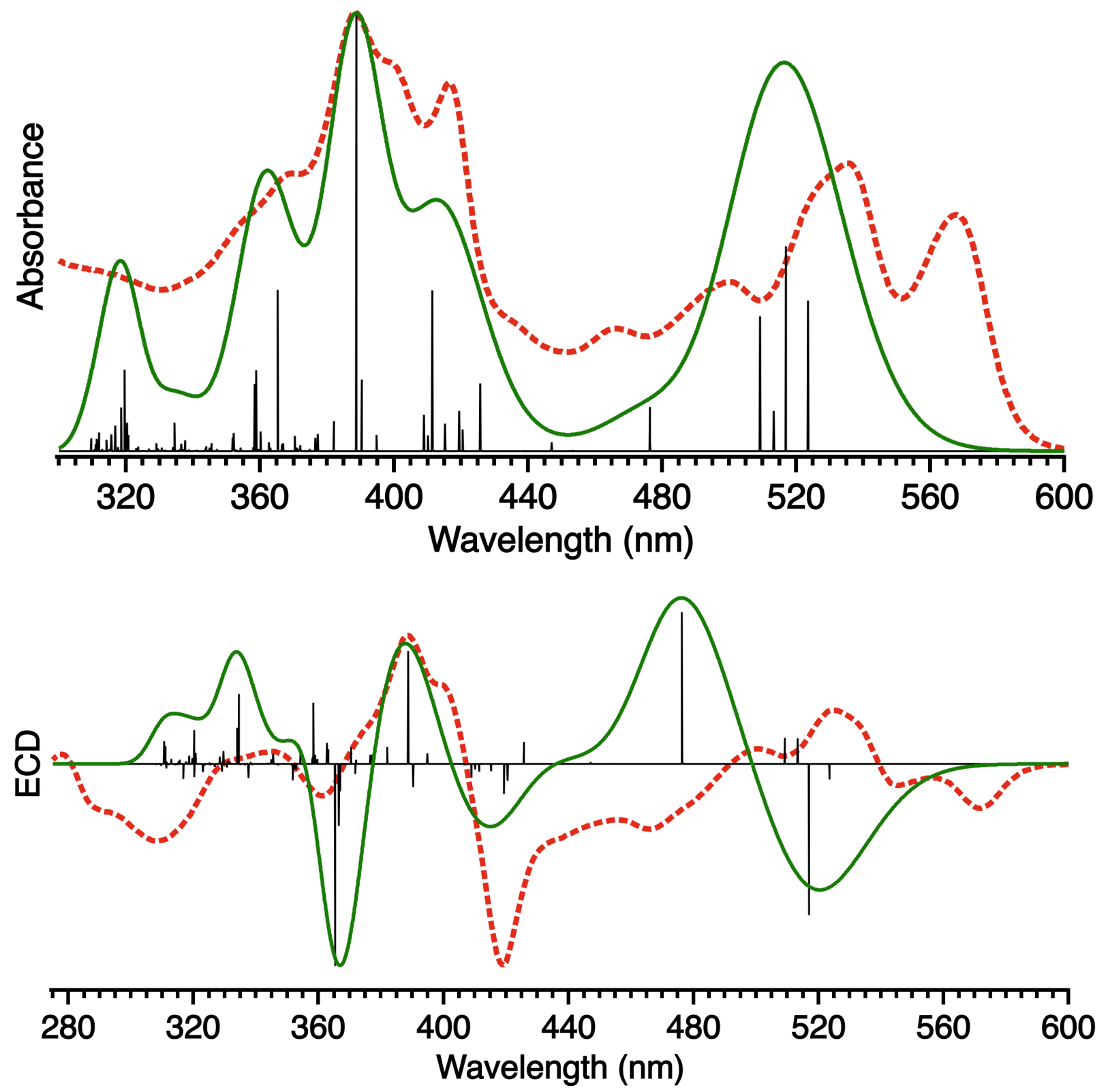

Figure S38. TDDFT-predicted (CAM-B3LYP/6-31++G**) UV-visible absorbance and ECD spectra of $\boldsymbol{P P}$ M[6]-PP (zero bandwidth in black, $\sigma=0.13 \mathrm{eV}$ in green) overlaid on the experimental spectra of WH[6] in dichloromethane $\left(10^{-6} \mathrm{M}, 1 \mathrm{~cm}\right.$ path length) at room temperature (dotted orange). A positive wavelength shift of $56 \mathrm{~nm}$ has been applied to the calculated spectra. The intensities of the bandbroadened calculated spectra have also been scaled by the following factors: UV-visible absorbance, $0.6614 ; \mathrm{ECD}, 0.4500$. 
Table S5. TDDFT-Calculated Singlet Excited State Data for PP-M[6]-PP†

\begin{tabular}{|c|c|c|c|c|c|}
\hline Excited State & $E(e V)$ & $\lambda(\mathrm{nm})$ & $\lambda+56(\mathrm{~nm})$ & f & $R^{r}\left(10^{-40}\right.$ cgs $)$ \\
\hline 1 & 2.652 & 467.51 & 523.51 & 0.6011 & -133.6061 \\
\hline 2 & 2.6902 & 460.87 & 516.87 & 0.8188 & -1281.2512 \\
\hline 3 & 2.7111 & 457.32 & 513.32 & 0.161 & 216.1122 \\
\hline 4 & 2.7356 & 453.22 & 509.22 & 0.5392 & 218.7007 \\
\hline 5 & 2.949 & 420.42 & 476.42 & 0.0329 & 26.3695 \\
\hline 6 & 2.9502 & 420.26 & 476.26 & 0.1768 & 1172.8675 \\
\hline 7 & 3.1199 & 397.4 & 453.4 & 0.0048 & 5.7549 \\
\hline 8 & 3.171 & 390.99 & 446.99 & 0.0348 & 14.7658 \\
\hline 9 & 3.3535 & 369.72 & 425.72 & 0.2704 & 147.6884 \\
\hline 10 & 3.4011 & 364.54 & 420.54 & 0.0853 & -114.5976 \\
\hline 11 & 3.4115 & 363.43 & 419.43 & 0.1598 & -202.8978 \\
\hline 12 & 3.4514 & 359.22 & 415.22 & 0.1107 & -47.156 \\
\hline 13 & 3.4886 & 355.39 & 411.39 & 0.6422 & -51.9548 \\
\hline 14 & 3.5012 & 354.12 & 410.12 & 0.0635 & -35.7671 \\
\hline 15 & 3.5129 & 352.94 & 408.94 & 0.1449 & -89.6941 \\
\hline 16 & 3.6593 & 338.82 & 394.82 & 0.064 & 65.9276 \\
\hline 17 & 3.7084 & 334.33 & 390.33 & 0.2871 & -143.9459 \\
\hline 18 & 3.727 & 332.67 & 388.67 & 1.7572 & 691.284 \\
\hline 19 & 3.8031 & 326.01 & 382.01 & 0.1187 & 102.0558 \\
\hline 20 & 3.8596 & 321.24 & 377.24 & 0.0688 & 52.9447 \\
\hline 21 & 3.8684 & 320.5 & 376.5 & 0.0523 & 54.335 \\
\hline 22 & 3.8896 & 318.76 & 374.76 & 0.007 & 0.82 \\
\hline 23 & 3.9241 & 315.95 & 371.95 & 0.0237 & 23.2951 \\
\hline 24 & 3.9256 & 315.83 & 371.83 & 0.0089 & -59.1482 \\
\hline 25 & 3.9364 & 314.96 & 370.96 & 0.0132 & 3.6827 \\
\hline 26 & 3.9434 & 314.41 & 370.41 & 0.0606 & 94.9166 \\
\hline 27 & 3.9876 & 310.92 & 366.92 & 0.0309 & -157.1912 \\
\hline 28 & 3.9925 & 310.54 & 366.54 & 0.0274 & -353.6417 \\
\hline 29 & 4.0086 & 309.3 & 365.3 & 0.6441 & -1147.463 \\
\hline 30 & 4.0375 & 307.08 & 363.08 & 0.0138 & 83.751 \\
\hline 31 & 4.0429 & 306.67 & 362.67 & 0.0362 & 117.5106 \\
\hline 32 & 4.0756 & 304.21 & 360.21 & 0.0776 & 2.9829 \\
\hline 33 & 4.0843 & 303.57 & 359.57 & 0.0073 & 28.8043 \\
\hline 34 & 4.0875 & 303.33 & 359.33 & 0.0054 & 19.2415 \\
\hline 35 & 4.0926 & 302.94 & 358.94 & 0.3237 & 51.0113 \\
\hline 36 & 4.0994 & 302.45 & 358.45 & 0.2681 & 339.667 \\
\hline 37 & 4.1012 & 302.31 & 358.31 & 0.0152 & 55.341 \\
\hline 38 & 4.1054 & 302 & 358 & 0.0151 & 32.1482 \\
\hline 39 & 4.1575 & 298.22 & 354.22 & 0.0142 & 64.3059 \\
\hline 40 & 4.1698 & 297.34 & 353.34 & 0.0003 & 3.4235 \\
\hline 41 & 4.1742 & 297.03 & 353.03 & 0.0004 & 4.4373 \\
\hline 42 & 4.178 & 296.75 & 352.75 & 0.0054 & -28.8649 \\
\hline 43 & 4.1812 & 296.53 & 352.53 & 0.0018 & 0.3682 \\
\hline 44 & 4.1877 & 296.07 & 352.07 & 0.0728 & -35.7512 \\
\hline 45 & 4.1903 & 295.88 & 351.88 & 0.0365 & -14.1139 \\
\hline 46 & 4.1917 & 295.79 & 351.79 & 0.0537 & -89.8428 \\
\hline 47 & 4.2592 & 291.1 & 347.1 & 0.0087 & -6.1665 \\
\hline 48 & 4.2831 & 289.47 & 345.47 & 0.0317 & 52.4968 \\
\hline 49 & 4.291 & 288.94 & 344.94 & 0.0135 & 25.345 \\
\hline 50 & 4.2976 & 288.49 & 344.49 & 0.001 & 3.1384 \\
\hline 51 & 4.3071 & 287.86 & 343.86 & 0.0194 & 4.1221 \\
\hline 52 & 4.3109 & 287.61 & 343.61 & 0.0077 & -0.6915 \\
\hline 53 & 4.3497 & 285.04 & 341.04 & 0.0043 & 0.607 \\
\hline 54 & 4.353 & 284.83 & 340.83 & 0.001 & -0.281 \\
\hline
\end{tabular}




\begin{tabular}{|c|c|c|c|c|c|}
\hline 55 & 4.3848 & 282.76 & 338.76 & 0.006 & -4.0432 \\
\hline 56 & 4.3916 & 282.32 & 338.32 & 0.0047 & 8.6342 \\
\hline 57 & 4.4028 & 281.6 & 337.6 & 0.0424 & -72.4593 \\
\hline 58 & 4.4107 & 281.1 & 337.1 & 0.0051 & 4.2632 \\
\hline 59 & 4.4206 & 280.47 & 336.47 & 0.0295 & -7.5303 \\
\hline 60 & 4.4295 & 279.9 & 335.9 & 0.0058 & -1.0619 \\
\hline 61 & 4.4449 & 278.93 & 334.93 & 0.001 & -3.8929 \\
\hline 62 & 4.4525 & 278.46 & 334.46 & 0.1131 & 357.3454 \\
\hline 63 & 4.4598 & 278.01 & 334.01 & 0.0162 & 183.4063 \\
\hline 64 & 4.4976 & 275.67 & 331.67 & 0.0035 & 7.4251 \\
\hline 65 & 4.5079 & 275.04 & 331.04 & 0.0019 & 16.4502 \\
\hline 66 & 4.5107 & 274.87 & 330.87 & 0.0018 & 6.9941 \\
\hline 67 & 4.5133 & 274.71 & 330.71 & 0.0132 & -18.2812 \\
\hline 68 & 4.5285 & 273.79 & 329.79 & 0.0015 & -2.7368 \\
\hline 69 & 4.5293 & 273.74 & 329.74 & 0.0003 & -0.0315 \\
\hline 70 & 4.532 & 273.58 & 329.58 & 0.0107 & 64.1554 \\
\hline 71 & 4.5399 & 273.1 & 329.1 & 0.0308 & -40.0236 \\
\hline 72 & 4.5518 & 272.38 & 328.38 & 0.0042 & 37.1686 \\
\hline 73 & 4.5589 & 271.96 & 327.96 & 0.0002 & 2.2094 \\
\hline 74 & 4.5773 & 270.87 & 326.87 & 0.0016 & 1.1862 \\
\hline 75 & 4.579 & 270.77 & 326.77 & 0.0093 & -13.1949 \\
\hline 76 & 4.5807 & 270.66 & 326.66 & 0.0003 & -5.1071 \\
\hline 77 & 4.597 & 269.71 & 325.71 & 0.0002 & -2.7562 \\
\hline 78 & 4.6013 & 269.45 & 325.45 & 0.0018 & -6.5517 \\
\hline 79 & 4.6074 & 269.1 & 325.1 & 0.001 & 7.4567 \\
\hline 80 & 4.6335 & 267.58 & 323.58 & 0.0185 & -5.8362 \\
\hline 81 & 4.6433 & 267.02 & 323.02 & 0.0119 & -40.0951 \\
\hline 82 & 4.6846 & 264.67 & 320.67 & 0.0638 & 52.9291 \\
\hline 83 & 4.6909 & 264.31 & 320.31 & 0.1146 & -64.6766 \\
\hline 84 & 4.6919 & 264.25 & 320.25 & 0.0115 & 164.5272 \\
\hline 85 & 4.7041 & 263.56 & 319.56 & 0.3246 & 28.2561 \\
\hline 86 & 4.7206 & 262.65 & 318.65 & 0.174 & 39.8621 \\
\hline 87 & 4.7387 & 261.64 & 317.64 & 0.0159 & 10.9232 \\
\hline 88 & 4.7539 & 260.8 & 316.8 & 0.102 & -71.8178 \\
\hline 89 & 4.7754 & 259.63 & 315.63 & 0.066 & 19.3495 \\
\hline 90 & 4.7842 & 259.16 & 315.16 & 0.0073 & 10.7927 \\
\hline 91 & 4.7897 & 258.86 & 314.86 & 0.0033 & -3.395 \\
\hline 92 & 4.8014 & 258.23 & 314.23 & 0.0444 & -6.0396 \\
\hline 93 & 4.8264 & 256.89 & 312.89 & 0.0084 & 23.532 \\
\hline 94 & 4.8457 & 255.86 & 311.86 & 0.0753 & -4.6896 \\
\hline 95 & 4.8548 & 255.38 & 311.38 & 0.0018 & 11.0546 \\
\hline 96 & 4.8561 & 255.31 & 311.31 & 0.0497 & -22.1551 \\
\hline 97 & 4.8613 & 255.04 & 311.04 & 0.0278 & 82.8624 \\
\hline 98 & 4.8696 & 254.61 & 310.61 & 0.0069 & 106.8912 \\
\hline 99 & 4.8899 & 253.55 & 309.55 & 0.05 & -8.0452 \\
\hline 100 & 4.9218 & 251.91 & 307.91 & 0.0013 & -3.3866 \\
\hline
\end{tabular}

tExcited state energies $(E)$, wavelengths $(\lambda)$, oscillator strengths $(f)$, and rotatory strengths in dipole length formalism $\left(R^{r}\right)$ were calculated at the CAM-B3LYP/6-31++ ${ }^{* *}$ level of theory. 


\section{b. WH[6][6]}
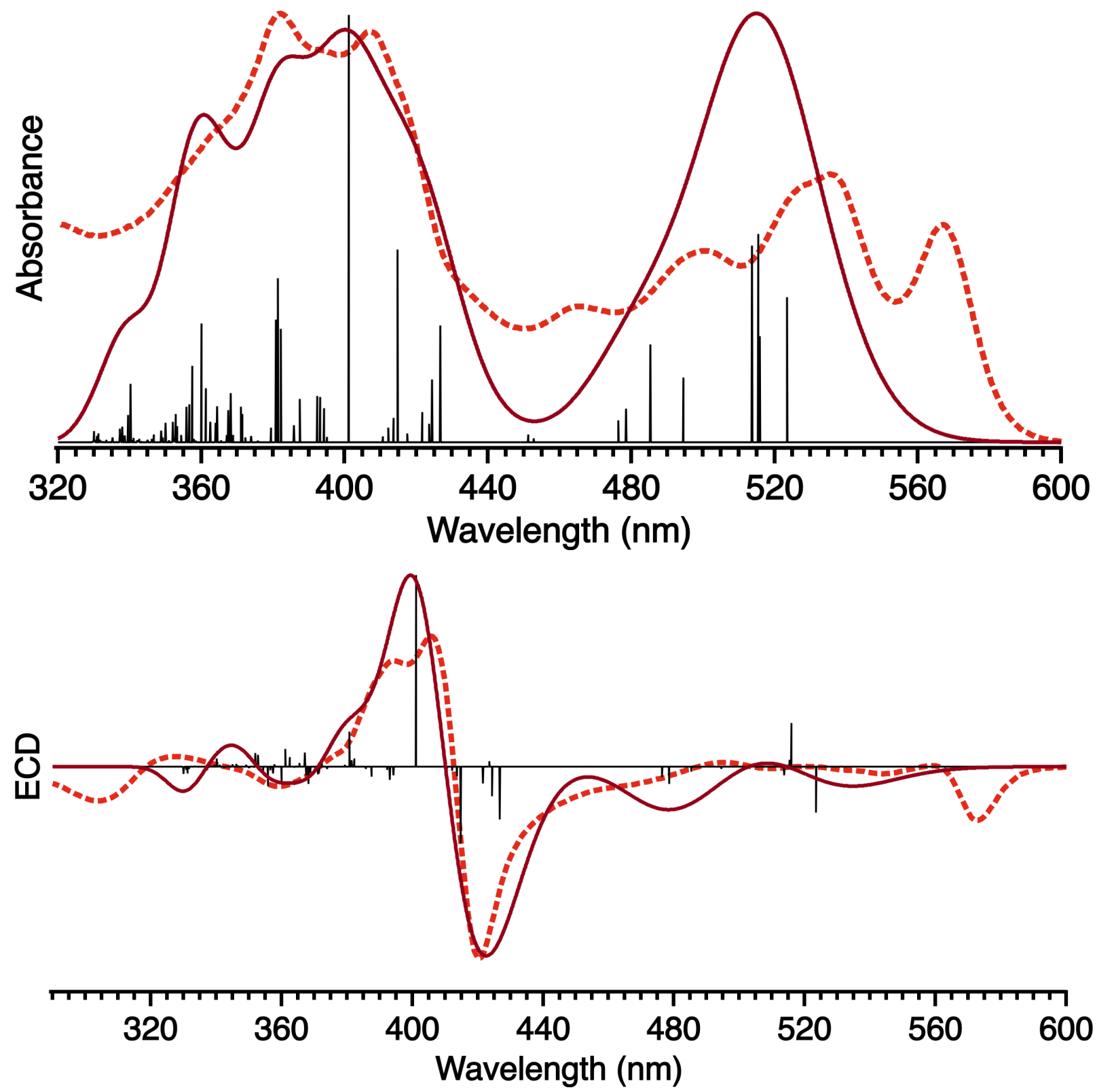

Figure S39. TDDFT-predicted (CAM-B3LYP/6-31++G**) UV-visible absorbance and ECD spectra of MM-M[6][6]-MM (zero bandwidth in black, $\sigma=0.13 \mathrm{eV}$ in red) overlaid on the experimental spectra of WH[6][6] in dichloromethane (10-6 M, $1 \mathrm{~cm}$ path length) at room temperature (dotted orange). A positive wavelength shift of $56 \mathrm{~nm}$ has been applied to the calculated spectra. The intensities of the bandbroadened calculated spectra have also been scaled by the following factors: UV-visible absorbance, 0.7438 ; ECD, 0.5672. 
Table S6. TDDFT-Calculated Singlet Excited State Data for $M M M-M[6][6]-M M+$

\begin{tabular}{|c|c|c|c|c|c|}
\hline Excited State & $E(e V)$ & $\lambda(\mathrm{nm})$ & $\lambda+56(\mathrm{~nm})$ & f & $R^{r}\left(10^{-40}\right.$ cgs $)$ \\
\hline 1 & 2.6519 & 467.53 & 523.53 & 0.4668 & -1968.1846 \\
\hline 2 & 2.696 & 459.89 & 515.89 & 0.3411 & 1849.5004 \\
\hline 3 & 2.6988 & 459.4 & 515.4 & 0.6709 & 287.7773 \\
\hline 4 & 2.7087 & 457.72 & 513.72 & 0.6337 & -372.1565 \\
\hline 5 & 2.8274 & 438.51 & 494.51 & 0.2085 & -82.9289 \\
\hline 6 & 2.8882 & 429.28 & 485.28 & 0.3155 & -193.2199 \\
\hline 7 & 2.9343 & 422.54 & 478.54 & 0.1078 & -675.7503 \\
\hline 8 & 2.949 & 420.43 & 476.43 & 0.0707 & -401.4269 \\
\hline 9 & 3.1246 & 396.8 & 452.8 & 0.0132 & 19.0422 \\
\hline 10 & 3.1374 & 395.18 & 451.18 & 0.0244 & -6.4414 \\
\hline 11 & 3.3449 & 370.67 & 426.67 & 0.3769 & -1799.6537 \\
\hline 12 & 3.365 & 368.45 & 424.45 & 0.2026 & -997.4601 \\
\hline 13 & 3.3723 & 367.65 & 423.65 & 0.0599 & 183.3005 \\
\hline 14 & 3.3917 & 365.55 & 421.55 & 0.0969 & -570.9252 \\
\hline 15 & 3.43 & 361.47 & 417.47 & 0.0282 & 1.9732 \\
\hline 16 & 3.4553 & 358.83 & 414.83 & 0.6203 & -2539.6645 \\
\hline 17 & 3.4669 & 357.63 & 413.63 & 0.0779 & -321.1345 \\
\hline 18 & 3.4807 & 356.2 & 412.2 & 0.0476 & -139.899 \\
\hline 19 & 3.4968 & 354.57 & 410.57 & 0.0195 & -63.6965 \\
\hline 20 & 3.593 & 345.08 & 401.08 & 1.3761 & 6087.096 \\
\hline 21 & 3.6578 & 338.96 & 394.96 & 0.0171 & 0.3227 \\
\hline 22 & 3.6661 & 338.2 & 394.2 & 0.1098 & -262.3467 \\
\hline 23 & 3.6784 & 337.06 & 393.06 & 0.1452 & -412.0743 \\
\hline 24 & 3.6866 & 336.31 & 392.31 & 0.149 & -95.3269 \\
\hline 25 & 3.7404 & 331.48 & 387.48 & 0.1392 & -305.6754 \\
\hline 26 & 3.7594 & 329.8 & 385.8 & 0.0545 & -70.968 \\
\hline 27 & 3.8003 & 326.25 & 382.25 & 0.3647 & 239.1457 \\
\hline 28 & 3.8099 & 325.43 & 381.43 & 0.529 & 192.5728 \\
\hline 29 & 3.8172 & 324.8 & 380.8 & 0.3943 & 1052.1283 \\
\hline 30 & 3.8341 & 323.38 & 379.38 & 0.0478 & 54.7192 \\
\hline 31 & 3.877 & 319.8 & 375.8 & 0.0047 & -5.6936 \\
\hline 32 & 3.9001 & 317.9 & 373.9 & 0.0211 & -72.6449 \\
\hline 33 & 3.9118 & 316.95 & 372.95 & 0.0014 & 13.9039 \\
\hline 34 & 3.9202 & 316.27 & 372.27 & 0.0154 & 184.7038 \\
\hline 35 & 3.9304 & 315.45 & 371.45 & 0.0915 & -199.0174 \\
\hline 36 & 3.9344 & 315.13 & 371.13 & 0.1147 & -228.6462 \\
\hline 37 & 3.9631 & 312.85 & 368.85 & 0.0229 & -162.1394 \\
\hline 38 & 3.971 & 312.23 & 368.23 & 0.159 & -499.7543 \\
\hline 39 & 3.98 & 311.52 & 367.52 & 0.1036 & -244.2842 \\
\hline 40 & 3.9854 & 311.1 & 367.1 & 0.0233 & 401.2079 \\
\hline 41 & 4.0057 & 309.52 & 365.52 & 0.0057 & 106.4846 \\
\hline 42 & 4.0205 & 308.38 & 364.38 & 0.1157 & 31.9405 \\
\hline 43 & 4.0262 & 307.95 & 363.95 & 0.0625 & -21.6463 \\
\hline 44 & 4.0448 & 306.53 & 362.53 & 0.0656 & 271.4556 \\
\hline 45 & 4.0619 & 305.24 & 361.24 & 0.1746 & 504.6675 \\
\hline 46 & 4.0786 & 303.99 & 359.99 & 0.3825 & -465.6213 \\
\hline 47 & 4.1002 & 302.39 & 358.39 & 0.0054 & 1.2101 \\
\hline 48 & 4.1058 & 301.97 & 357.97 & 0.0009 & -3.9536 \\
\hline 49 & 4.1069 & 301.9 & 357.9 & 0.0109 & 1.9486 \\
\hline 50 & 4.1084 & 301.78 & 357.78 & 0.0052 & 58.3925 \\
\hline 51 & 4.1134 & 301.42 & 357.42 & 0.2454 & -184.1531 \\
\hline 52 & 4.1251 & 300.56 & 356.56 & 0.1227 & -97.0898 \\
\hline 53 & 4.1358 & 299.79 & 355.79 & 0.1149 & -539.9838 \\
\hline 54 & 4.1554 & 298.37 & 354.37 & 0.0234 & -151.9597 \\
\hline
\end{tabular}




\begin{tabular}{|c|c|c|c|c|c|}
\hline 55 & 4.1604 & 298.01 & 354.01 & 0.0022 & -11.3483 \\
\hline 56 & 4.1712 & 297.24 & 353.24 & 0.0517 & -96.2501 \\
\hline 57 & 4.1774 & 296.79 & 352.79 & 0.0915 & 318.9988 \\
\hline 58 & 4.18 & 296.61 & 352.61 & 0.0045 & 25.0132 \\
\hline 59 & 4.184 & 296.33 & 352.33 & 0.014 & 29.09 \\
\hline 60 & 4.1889 & 295.98 & 351.98 & 0.0665 & 367.9312 \\
\hline 61 & 4.1923 & 295.74 & 351.74 & 0.002 & -15.8336 \\
\hline 62 & 4.2026 & 295.02 & 351.02 & 0.0058 & 16.5355 \\
\hline 63 & 4.2062 & 294.77 & 350.77 & 0.0005 & -6.1084 \\
\hline 64 & 4.2164 & 294.05 & 350.05 & 0.0623 & -32.6051 \\
\hline 65 & 4.2286 & 293.2 & 349.2 & 0.0152 & 46.9754 \\
\hline 66 & 4.2342 & 292.82 & 348.82 & 0.0375 & 1.0703 \\
\hline 67 & 4.245 & 292.07 & 348.07 & 0.0004 & -6.0604 \\
\hline 68 & 4.2647 & 290.72 & 346.72 & 0.025 & 22.7143 \\
\hline 69 & 4.272 & 290.23 & 346.23 & 0.0116 & 70.4932 \\
\hline 70 & 4.2899 & 289.01 & 345.01 & 0.0083 & 56.9776 \\
\hline 71 & 4.3007 & 288.29 & 344.29 & 0.0001 & -0.0446 \\
\hline 72 & 4.3029 & 288.14 & 344.14 & 0.0002 & -1.4229 \\
\hline 73 & 4.3152 & 287.32 & 343.32 & 0.0037 & 12.8963 \\
\hline 74 & 4.3261 & 286.6 & 342.6 & 0.0102 & -23.5394 \\
\hline 75 & 4.3285 & 286.44 & 342.44 & 0.0066 & -20.794 \\
\hline 76 & 4.3339 & 286.08 & 342.08 & 0.0059 & -5.1327 \\
\hline 77 & 4.3486 & 285.12 & 341.12 & 0.0147 & 50.8951 \\
\hline 78 & 4.3565 & 284.6 & 340.6 & 0.0068 & 19.6276 \\
\hline 79 & 4.3632 & 284.16 & 340.16 & 0.1883 & 219.5952 \\
\hline 80 & 4.374 & 283.46 & 339.46 & 0.0881 & 52.8353 \\
\hline 81 & 4.3867 & 282.64 & 338.64 & 0.0216 & 23.615 \\
\hline 82 & 4.3889 & 282.5 & 338.5 & 0.0042 & 1.1843 \\
\hline 83 & 4.3981 & 281.9 & 337.9 & 0.0501 & -4.066 \\
\hline 84 & 4.4048 & 281.48 & 337.48 & 0.0071 & -39.4702 \\
\hline 85 & 4.407 & 281.33 & 337.33 & 0.0439 & 36.829 \\
\hline 86 & 4.4095 & 281.18 & 337.18 & 0.0244 & -94.0737 \\
\hline 87 & 4.4234 & 280.29 & 336.29 & 0.0021 & -5.8403 \\
\hline 88 & 4.4415 & 279.15 & 335.15 & 0.0155 & 4.4313 \\
\hline 89 & 4.4486 & 278.71 & 334.71 & 0.0025 & -11.1277 \\
\hline 90 & 4.468 & 277.49 & 333.49 & 0.0076 & 12.8894 \\
\hline 91 & 4.4817 & 276.64 & 332.64 & 0.0014 & -3.6047 \\
\hline 92 & 4.4907 & 276.09 & 332.09 & 0.0031 & 4.6085 \\
\hline 93 & 4.4976 & 275.67 & 331.67 & 0.0075 & -52.0442 \\
\hline 94 & 4.5049 & 275.22 & 331.22 & 0.0289 & -170.117 \\
\hline 95 & 4.5119 & 274.79 & 330.79 & 0.02 & -78.98 \\
\hline 96 & 4.5201 & 274.3 & 330.3 & 0.0081 & -15.6797 \\
\hline 97 & 4.5238 & 274.07 & 330.07 & 0.0101 & 10.9336 \\
\hline 98 & 4.5248 & 274.01 & 330.01 & 0.0362 & -186.4691 \\
\hline 99 & 4.5287 & 273.77 & 329.77 & 0.0013 & -13.4814 \\
\hline 100 & 4.5315 & 273.6 & 329.6 & 0.0025 & 0.6579 \\
\hline
\end{tabular}

tExcited state energies $(E)$, wavelengths $(\lambda)$, oscillator strengths $(f)$, and rotatory strengths in dipole length formalism $\left(R^{r}\right)$ were calculated at the CAM-B3LYP/6-31++ ${ }^{* *}$ level of theory. 

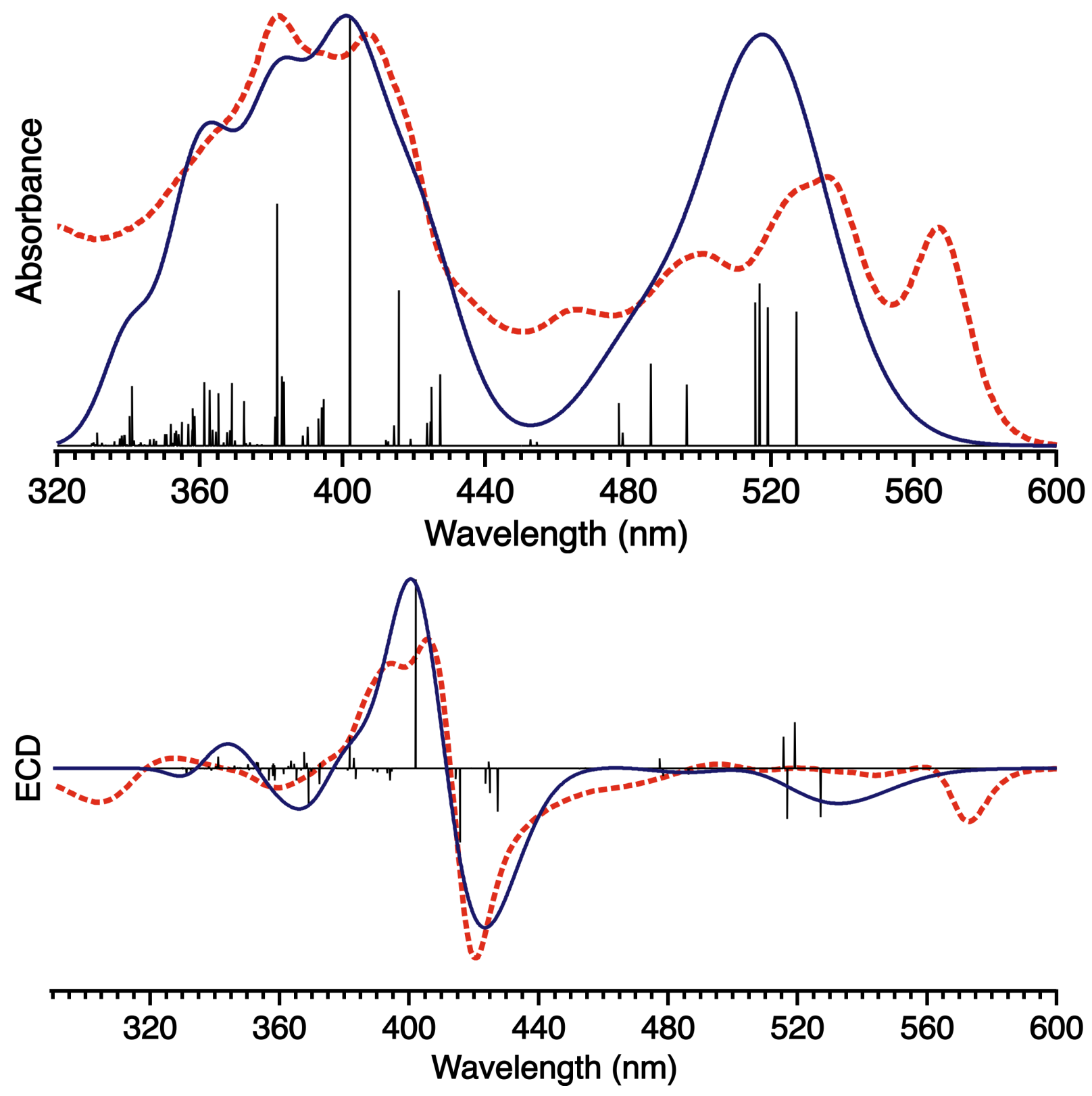

Figure S40. TDDFT-predicted (CAM-B3LYP/6-31++ $\mathrm{G}^{\star \star}$ ) UV-visible absorbance and ECD spectra of MM-M[6][6]-PP (zero bandwidth in black, $\sigma=0.13 \mathrm{eV}$ in blue) overlaid on the experimental spectra of WH[6][6] in dichloromethane (10-6 M, $1 \mathrm{~cm}$ path length) at room temperature (dotted orange). A positive wavelength shift of $56 \mathrm{~nm}$ has been applied to the calculated spectra. The intensities of the bandbroadened calculated spectra have also been scaled by the following factors: UV-visible absorbance, 0.7453 ; ECD, 0.5331. 
Table S7. TDDFT-Calculated Singlet Excited State Data for $\mathbf{M M - M [ 6 ] [ 6 ] - P P \dagger}$

\begin{tabular}{|c|c|c|c|c|c|}
\hline Excited State & $E(e V)$ & $\lambda(\mathrm{nm})$ & $\lambda+56(\mathrm{~nm})$ & $f$ & $R^{r}\left(10^{-40} \mathrm{cgs}\right)$ \\
\hline 1 & 2.6319 & 471.07 & 527.07 & 0.4729 & -2214.849 \\
\hline 2 & 2.6775 & 463.06 & 519.06 & 0.4899 & 2047.6088 \\
\hline 3 & 2.6908 & 460.78 & 516.78 & 0.5736 & -2245.0228 \\
\hline 4 & 2.6978 & 459.58 & 515.58 & 0.507 & 1400.5583 \\
\hline 5 & 2.8153 & 440.4 & 496.4 & 0.2156 & 25.9183 \\
\hline 6 & 2.8816 & 430.26 & 486.26 & 0.2894 & -263.6139 \\
\hline 7 & 2.9355 & 422.36 & 478.36 & 0.0467 & -316.325 \\
\hline 8 & 2.942 & 421.42 & 477.42 & 0.1518 & 412.8493 \\
\hline 9 & 3.112 & 398.41 & 454.41 & 0.0143 & 23.8657 \\
\hline 10 & 3.1266 & 396.55 & 452.55 & 0.0219 & -13.3593 \\
\hline 11 & 3.3389 & 371.33 & 427.33 & 0.2525 & -1557.8054 \\
\hline 12 & 3.3608 & 368.92 & 424.92 & 0.2078 & -876.7246 \\
\hline 13 & 3.3638 & 368.58 & 424.58 & 0.0866 & 236.9285 \\
\hline 14 & 3.3724 & 367.65 & 423.65 & 0.0812 & -537.2185 \\
\hline 15 & 3.416 & 362.95 & 418.95 & 0.0239 & 19.77 \\
\hline 16 & 3.4465 & 359.74 & 415.74 & 0.5489 & -2572.3287 \\
\hline 17 & 3.4589 & 358.45 & 414.45 & 0.0721 & -381.6439 \\
\hline 18 & 3.4755 & 356.74 & 412.74 & 0.0152 & -34.2522 \\
\hline 19 & 3.4819 & 356.09 & 412.09 & 0.0201 & -66.0693 \\
\hline 20 & 3.5838 & 345.96 & 401.96 & 1.5149 & 6305.4131 \\
\hline 21 & 3.649 & 339.77 & 395.77 & 0.0012 & 13.2418 \\
\hline 22 & 3.6617 & 338.6 & 394.6 & 0.1652 & -106.8068 \\
\hline 23 & 3.6674 & 338.07 & 394.07 & 0.1352 & -391.5885 \\
\hline 24 & 3.6764 & 337.24 & 393.24 & 0.0967 & -164.7882 \\
\hline 25 & 3.7096 & 334.22 & 390.22 & 0.0666 & -130.504 \\
\hline 26 & 3.7256 & 332.79 & 388.79 & 0.0368 & -88.9775 \\
\hline 27 & 3.7857 & 327.51 & 383.51 & 0.2273 & -347.1606 \\
\hline 28 & 3.7922 & 326.94 & 382.94 & 0.2448 & 333.8096 \\
\hline 29 & 3.8076 & 325.63 & 381.63 & 0.855 & 996.5746 \\
\hline 30 & 3.8146 & 325.02 & 381.02 & 0.1025 & -71.7118 \\
\hline 31 & 3.8587 & 321.31 & 377.31 & 0.005 & 45.1937 \\
\hline 32 & 3.8751 & 319.95 & 375.95 & 0.0044 & 7.7336 \\
\hline 33 & 3.8988 & 318.01 & 374.01 & 0.0115 & 14.9594 \\
\hline 34 & 3.9122 & 316.92 & 372.92 & 0.0092 & -1.4345 \\
\hline 35 & 3.9182 & 316.43 & 372.43 & 0.0847 & 161.3195 \\
\hline 36 & 3.9199 & 316.3 & 372.3 & 0.158 & -485.2905 \\
\hline 37 & 3.9508 & 313.82 & 369.82 & 0.0195 & -100.7585 \\
\hline 38 & 3.962 & 312.93 & 368.93 & 0.2219 & -1070.9518 \\
\hline 39 & 3.9693 & 312.36 & 368.36 & 0.0541 & 164.2505 \\
\hline 40 & 3.9791 & 311.59 & 367.59 & 0.0475 & 495.0134 \\
\hline 41 & 3.9919 & 310.59 & 366.59 & 0.0114 & -63.4318 \\
\hline 42 & 4.0094 & 309.24 & 365.24 & 0.1857 & -362.9682 \\
\hline 43 & 4.0193 & 308.47 & 364.47 & 0.0493 & 130.4002 \\
\hline 44 & 4.0323 & 307.47 & 363.47 & 0.0572 & 222.7223 \\
\hline 45 & 4.0428 & 306.68 & 362.68 & 0.1968 & 65.7593 \\
\hline 46 & 4.062 & 305.23 & 361.23 & 0.225 & -173.2918 \\
\hline 47 & 4.0984 & 302.52 & 358.52 & 0.1049 & -357.4242 \\
\hline 48 & 4.1016 & 302.28 & 358.28 & 0.0482 & 92.1874 \\
\hline 49 & 4.1047 & 302.05 & 358.05 & 0.0303 & -96.6837 \\
\hline 50 & 4.1058 & 301.97 & 357.97 & 0.1012 & 246.0855 \\
\hline 51 & 4.1071 & 301.88 & 357.88 & 0.0607 & -226.3264 \\
\hline 52 & 4.1089 & 301.75 & 357.75 & 0.0519 & 87.324 \\
\hline 53 & 4.1228 & 300.73 & 356.73 & 0.0777 & -353.1945 \\
\hline 54 & 4.1481 & 298.9 & 354.9 & 0.0847 & 10.1749 \\
\hline
\end{tabular}




\begin{tabular}{|c|c|c|c|c|c|}
\hline 55 & 4.1574 & 298.22 & 354.22 & 0.0176 & -2.0287 \\
\hline 56 & 4.1611 & 297.96 & 353.96 & 0.0405 & -95.0803 \\
\hline 57 & 4.1701 & 297.32 & 353.32 & 0.0534 & 167.3206 \\
\hline 58 & 4.1728 & 297.12 & 353.12 & 0.0165 & 79.562 \\
\hline 59 & 4.1758 & 296.91 & 352.91 & 0.0442 & 181.7528 \\
\hline 60 & 4.1796 & 296.64 & 352.64 & 0.0009 & 0.8569 \\
\hline 61 & 4.1849 & 296.26 & 352.26 & 0.0027 & -0.8613 \\
\hline 62 & 4.1858 & 296.2 & 352.2 & 0.0103 & 21.5492 \\
\hline 63 & 4.192 & 295.76 & 351.76 & 0.078 & -26.9799 \\
\hline 64 & 4.2088 & 294.59 & 350.59 & 0.0409 & -40.5571 \\
\hline 65 & 4.2095 & 294.54 & 350.54 & 0.009 & -44.048 \\
\hline 66 & 4.2151 & 294.15 & 350.15 & 0.0409 & 121.4291 \\
\hline 67 & 4.245 & 292.07 & 348.07 & 0.0019 & 11.8473 \\
\hline 68 & 4.2501 & 291.72 & 347.72 & 0.0173 & 23.7749 \\
\hline 69 & 4.2612 & 290.96 & 346.96 & 0.024 & 26.3608 \\
\hline 70 & 4.2752 & 290.01 & 346.01 & 0.0217 & 77.3062 \\
\hline 71 & 4.2959 & 288.61 & 344.61 & 0.0001 & 0.4582 \\
\hline 72 & 4.297 & 288.54 & 344.54 & 0.0028 & 12.6315 \\
\hline 73 & 4.2993 & 288.38 & 344.38 & 0.0017 & 6.1067 \\
\hline 74 & 4.3079 & 287.81 & 343.81 & 0.0009 & -10.5337 \\
\hline 75 & 4.3143 & 287.38 & 343.38 & 0.0125 & -4.4081 \\
\hline 76 & 4.3214 & 286.91 & 342.91 & 0.0056 & -2.5157 \\
\hline 77 & 4.3421 & 285.54 & 341.54 & 0.0196 & 25.1549 \\
\hline 78 & 4.3469 & 285.22 & 341.22 & 0.0033 & 12.2622 \\
\hline 79 & 4.3506 & 284.98 & 340.98 & 0.2108 & 325.5353 \\
\hline 80 & 4.361 & 284.3 & 340.3 & 0.1048 & 52.1791 \\
\hline 81 & 4.3734 & 283.5 & 339.5 & 0.0059 & 2.0856 \\
\hline 82 & 4.3824 & 282.91 & 338.91 & 0.0371 & -64.8337 \\
\hline 83 & 4.3856 & 282.71 & 338.71 & 0.0379 & -5.6851 \\
\hline 84 & 4.3947 & 282.12 & 338.12 & 0.008 & -13.9449 \\
\hline 85 & 4.3954 & 282.08 & 338.08 & 0.0277 & 63.4682 \\
\hline 86 & 4.4049 & 281.47 & 337.47 & 0.0265 & -13.5424 \\
\hline 87 & 4.4146 & 280.85 & 336.85 & 0.0012 & -8.6742 \\
\hline 88 & 4.4275 & 280.03 & 336.03 & 0.0158 & 5.6699 \\
\hline 89 & 4.4386 & 279.33 & 335.33 & 0.0012 & -15.0082 \\
\hline 90 & 4.4566 & 278.2 & 334.2 & 0.0009 & 3.5259 \\
\hline 91 & 4.4677 & 277.51 & 333.51 & 0.003 & -0.3503 \\
\hline 92 & 4.4812 & 276.68 & 332.68 & 0.0035 & -5.2569 \\
\hline 93 & 4.4842 & 276.49 & 332.49 & 0.0096 & -56.5194 \\
\hline 94 & 4.4946 & 275.85 & 331.85 & 0.0021 & 3.3132 \\
\hline 95 & 4.5056 & 275.18 & 331.18 & 0.0455 & -167.1031 \\
\hline 96 & 4.5095 & 274.94 & 330.94 & 0.0051 & -3.6252 \\
\hline 97 & 4.5222 & 274.17 & 330.17 & 0.0112 & 28.0299 \\
\hline 98 & 4.5245 & 274.03 & 330.03 & 0.0022 & -22.3644 \\
\hline 99 & 4.5273 & 273.86 & 329.86 & 0.005 & -3.8853 \\
\hline 100 & 4.5297 & 273.71 & 329.71 & 0.0088 & 11.5706 \\
\hline
\end{tabular}

tExcited state energies $(E)$, wavelengths $(\lambda)$, oscillator strengths $(f)$, and rotatory strengths in dipole length formalism $\left(R^{r}\right)$ were calculated at the CAM-B3LYP/6-31++ ${ }^{* *}$ level of theory. 

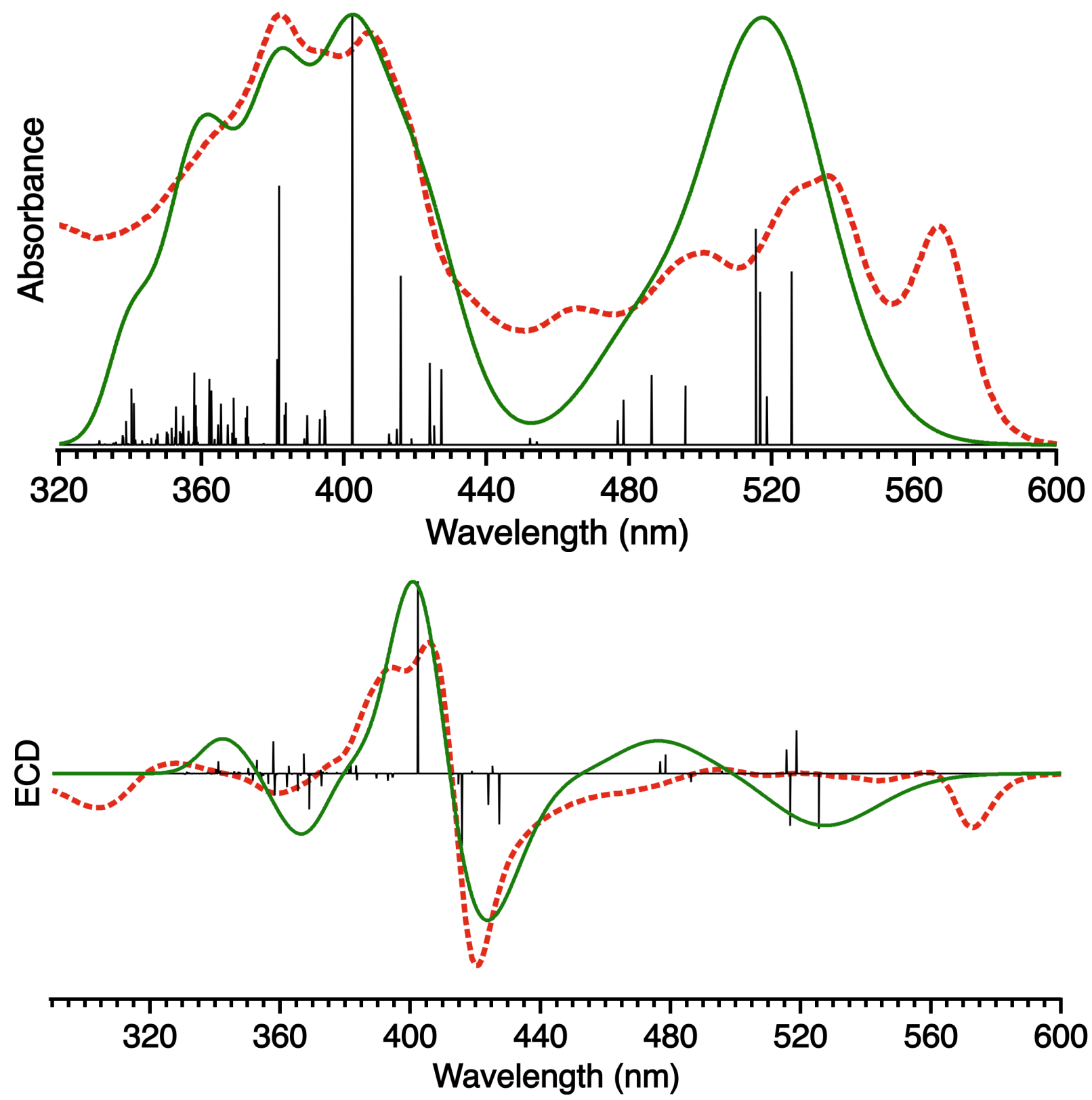

Figure S41. TDDFT-predicted (CAM-B3LYP/6-31++G**) UV-visible absorbance and ECD spectra of $\boldsymbol{P P}$ M[6][6]-PP (zero bandwidth in black, $\sigma=0.13 \mathrm{eV}$ in green) overlaid on the experimental spectra of WH[6][6] in dichloromethane (10-6 M, $1 \mathrm{~cm}$ path length) at room temperature (dotted orange). A positive wavelength shift of $56 \mathrm{~nm}$ has been applied to the calculated spectra. The intensities of the bandbroadened calculated spectra have also been scaled by the following factors: UV-visible absorbance, 0.7737; ECD, 0.5014. 
Table S8. TDDFT-Calculated Singlet Excited State Data for PP-M[6][6]-PP†

\begin{tabular}{|c|c|c|c|c|c|}
\hline Excited State & $E(e V)$ & $\lambda(\mathrm{nm})$ & $\lambda+56(\mathrm{~nm})$ & f & $R^{r}\left(10^{-40} \mathrm{cgs}\right)$ \\
\hline 1 & 2.6403 & 469.57 & 525.57 & 0.6014 & -2537.8231 \\
\hline 2 & 2.6798 & 462.67 & 518.67 & 0.168 & 1951.0169 \\
\hline 3 & 2.6908 & 460.77 & 516.77 & 0.5308 & -2356.3026 \\
\hline 4 & 2.6973 & 459.65 & 515.65 & 0.7502 & 1085.0731 \\
\hline 5 & 2.8188 & 439.85 & 495.85 & 0.2043 & 124.7449 \\
\hline 6 & 2.8812 & 430.32 & 486.32 & 0.2426 & -364.3817 \\
\hline 7 & 2.935 & 422.43 & 478.43 & 0.1556 & 788.6678 \\
\hline 8 & 2.9462 & 420.82 & 476.82 & 0.085 & 513.2364 \\
\hline 9 & 3.1147 & 398.06 & 454.06 & 0.0119 & 26.1903 \\
\hline 10 & 3.1292 & 396.21 & 452.21 & 0.0229 & -16.696 \\
\hline 11 & 3.3395 & 371.27 & 427.27 & 0.2616 & -1850.8639 \\
\hline 12 & 3.3575 & 369.28 & 425.28 & 0.0677 & 280.3353 \\
\hline 13 & 3.3678 & 368.15 & 424.15 & 0.0444 & 7.1887 \\
\hline 14 & 3.3694 & 367.97 & 423.97 & 0.2834 & -1135.0542 \\
\hline 15 & 3.4166 & 362.88 & 418.88 & 0.0219 & 95.1461 \\
\hline 16 & 3.4446 & 359.94 & 415.94 & 0.5863 & -2542.6596 \\
\hline 17 & 3.4554 & 358.81 & 414.81 & 0.0558 & -379.6511 \\
\hline 18 & 3.4717 & 357.13 & 413.13 & 0.0076 & -38.2285 \\
\hline 19 & 3.4763 & 356.65 & 412.65 & 0.0382 & -66.4951 \\
\hline 20 & 3.5806 & 346.27 & 402.27 & 1.4899 & 6500.953 \\
\hline 21 & 3.6459 & 340.06 & 396.06 & 0.001 & -10.7669 \\
\hline 22 & 3.6601 & 338.74 & 394.74 & 0.0987 & -119.2638 \\
\hline 23 & 3.6626 & 338.51 & 394.51 & 0.1203 & -136.7795 \\
\hline 24 & 3.6778 & 337.11 & 393.11 & 0.089 & -242.9823 \\
\hline 25 & 3.7161 & 333.64 & 389.64 & 0.1032 & -158.5993 \\
\hline 26 & 3.7253 & 332.81 & 388.81 & 0.0213 & -21.6282 \\
\hline 27 & 3.7852 & 327.55 & 383.55 & 0.1463 & -221.7752 \\
\hline 28 & 3.7885 & 327.26 & 383.26 & 0.1046 & 259.6147 \\
\hline 29 & 3.8071 & 325.67 & 381.67 & 0.8989 & 383.6049 \\
\hline 30 & 3.8127 & 325.19 & 381.19 & 0.2967 & 147.5136 \\
\hline 31 & 3.8578 & 321.38 & 377.38 & 0.0044 & 38.0428 \\
\hline 32 & 3.8705 & 320.33 & 376.33 & 0.0009 & 5.9566 \\
\hline 33 & 3.8948 & 318.34 & 374.34 & 0.0019 & 41.5983 \\
\hline 34 & 3.9101 & 317.08 & 373.08 & 0.0281 & 59.4815 \\
\hline 35 & 3.9146 & 316.72 & 372.72 & 0.1342 & -390.4092 \\
\hline 36 & 3.9184 & 316.42 & 372.42 & 0.0943 & 111.6758 \\
\hline 37 & 3.9529 & 313.65 & 369.65 & 0.0227 & -58.9395 \\
\hline 38 & 3.9609 & 313.02 & 369.02 & 0.1626 & -1104.4538 \\
\hline 39 & 3.9661 & 312.61 & 368.61 & 0.0422 & -64.9481 \\
\hline 40 & 3.9823 & 311.34 & 367.34 & 0.0714 & 602.1562 \\
\hline 41 & 3.9954 & 310.32 & 366.32 & 0.003 & -66.2996 \\
\hline 42 & 4.0077 & 309.36 & 365.36 & 0.143 & -538.682 \\
\hline 43 & 4.0183 & 308.55 & 364.55 & 0.0713 & 1.5464 \\
\hline 44 & 4.0305 & 307.61 & 363.61 & 0.0195 & -25.7761 \\
\hline 45 & 4.0428 & 306.68 & 362.68 & 0.1886 & 228.2632 \\
\hline 46 & 4.0508 & 306.07 & 362.07 & 0.2294 & -405.2138 \\
\hline 47 & 4.0932 & 302.9 & 358.9 & 0.0103 & -45.6838 \\
\hline 48 & 4.0986 & 302.5 & 358.5 & 0.0077 & 11.8641 \\
\hline 49 & 4.099 & 302.47 & 358.47 & 0.0567 & -183.106 \\
\hline 50 & 4.102 & 302.26 & 358.26 & 0.138 & -643.6755 \\
\hline 51 & 4.1064 & 301.93 & 357.93 & 0.251 & 952.9542 \\
\hline 52 & 4.1094 & 301.71 & 357.71 & 0.0109 & -19.9999 \\
\hline 53 & 4.1286 & 300.31 & 356.31 & 0.0494 & -306.2774 \\
\hline 54 & 4.1492 & 298.81 & 354.81 & 0.1005 & -69.7104 \\
\hline
\end{tabular}




\begin{tabular}{|c|c|c|c|c|c|}
\hline 55 & 4.1579 & 298.19 & 354.19 & 0.04 & -163.9792 \\
\hline 56 & 4.1624 & 297.87 & 353.87 & 0.0478 & 33.726 \\
\hline 57 & 4.1652 & 297.67 & 353.67 & 0.0013 & -4.0093 \\
\hline 58 & 4.1665 & 297.57 & 353.57 & 0.0016 & -23.8955 \\
\hline 59 & 4.1775 & 296.79 & 352.79 & 0.1332 & 400.0718 \\
\hline 60 & 4.1821 & 296.47 & 352.47 & 0.0266 & 88.8813 \\
\hline 61 & 4.1915 & 295.8 & 351.8 & 0.0004 & 4.5865 \\
\hline 62 & 4.1929 & 295.7 & 351.7 & 0.001 & 6.623 \\
\hline 63 & 4.1943 & 295.6 & 351.6 & 0.0596 & -198.1058 \\
\hline 64 & 4.2093 & 294.55 & 350.55 & 0.0108 & 22.8877 \\
\hline 65 & 4.2101 & 294.49 & 350.49 & 0.0394 & -56.4742 \\
\hline 66 & 4.2143 & 294.2 & 350.2 & 0.0454 & 162.4716 \\
\hline 67 & 4.2476 & 291.89 & 347.89 & 0.0004 & -6.4995 \\
\hline 68 & 4.2522 & 291.58 & 347.58 & 0.0382 & 37.1898 \\
\hline 69 & 4.2576 & 291.21 & 347.21 & 0.0179 & 45.9509 \\
\hline 70 & 4.277 & 289.89 & 345.89 & 0.0233 & 65.0559 \\
\hline 71 & 4.2934 & 288.78 & 344.78 & 0.005 & 24.7978 \\
\hline 72 & 4.2938 & 288.75 & 344.75 & 0.0002 & 2.7751 \\
\hline 73 & 4.2974 & 288.51 & 344.51 & 0.0019 & 3.7943 \\
\hline 74 & 4.3063 & 287.91 & 343.91 & 0.0009 & -1.2582 \\
\hline 75 & 4.3152 & 287.32 & 343.32 & 0.0152 & 3.7167 \\
\hline 76 & 4.3223 & 286.84 & 342.84 & 0.0027 & 1.2483 \\
\hline 77 & 4.3438 & 285.43 & 341.43 & 0.0187 & 82.0954 \\
\hline 78 & 4.3451 & 285.34 & 341.34 & 0.0125 & 10.2639 \\
\hline 79 & 4.3511 & 284.95 & 340.95 & 0.1452 & 337.8836 \\
\hline 80 & 4.3609 & 284.31 & 340.31 & 0.1947 & 123.4917 \\
\hline 81 & 4.3741 & 283.45 & 339.45 & 0.0051 & 5.0887 \\
\hline 82 & 4.3811 & 283 & 339 & 0.0012 & -24.5413 \\
\hline 83 & 4.3843 & 282.79 & 338.79 & 0.0826 & -5.1607 \\
\hline 84 & 4.3959 & 282.05 & 338.05 & 0.0265 & 14.7867 \\
\hline 85 & 4.3994 & 281.82 & 337.82 & 0.0343 & 28.9997 \\
\hline 86 & 4.4061 & 281.39 & 337.39 & 0.0011 & 19.2807 \\
\hline 87 & 4.4156 & 280.79 & 336.79 & 0.001 & -1.8858 \\
\hline 88 & 4.4295 & 279.9 & 335.9 & 0.0106 & 1.3367 \\
\hline 89 & 4.4407 & 279.2 & 335.2 & 0.0063 & -16.2857 \\
\hline 90 & 4.4605 & 277.96 & 333.96 & 0.0006 & -0.1464 \\
\hline 91 & 4.4674 & 277.53 & 333.53 & 0.0016 & 9.3198 \\
\hline 92 & 4.4797 & 276.77 & 332.77 & 0.0038 & -7.6356 \\
\hline 93 & 4.4934 & 275.93 & 331.93 & 0.0006 & 21.1738 \\
\hline 94 & 4.4958 & 275.78 & 331.78 & 0.0003 & -0.8932 \\
\hline 95 & 4.5041 & 275.27 & 331.27 & 0.0153 & 45.7239 \\
\hline 96 & 4.5123 & 274.77 & 330.77 & 0.0026 & -24.8981 \\
\hline 97 & 4.5169 & 274.49 & 330.49 & 0.0011 & -16.5045 \\
\hline 98 & 4.5195 & 274.33 & 330.33 & 0.0021 & 6.9269 \\
\hline 99 & 4.5277 & 273.84 & 329.84 & 0.0005 & 0.5351 \\
\hline 100 & 4.5318 & 273.59 & 329.59 & 0.0003 & -0.691 \\
\hline
\end{tabular}

tExcited state energies $(E)$, wavelengths $(\lambda)$, oscillator strengths $(f)$, and rotatory strengths in dipole length formalism $\left(R^{r}\right)$ were calculated at the CAM-B3LYP/6-31++ ${ }^{* *}$ level of theory. 

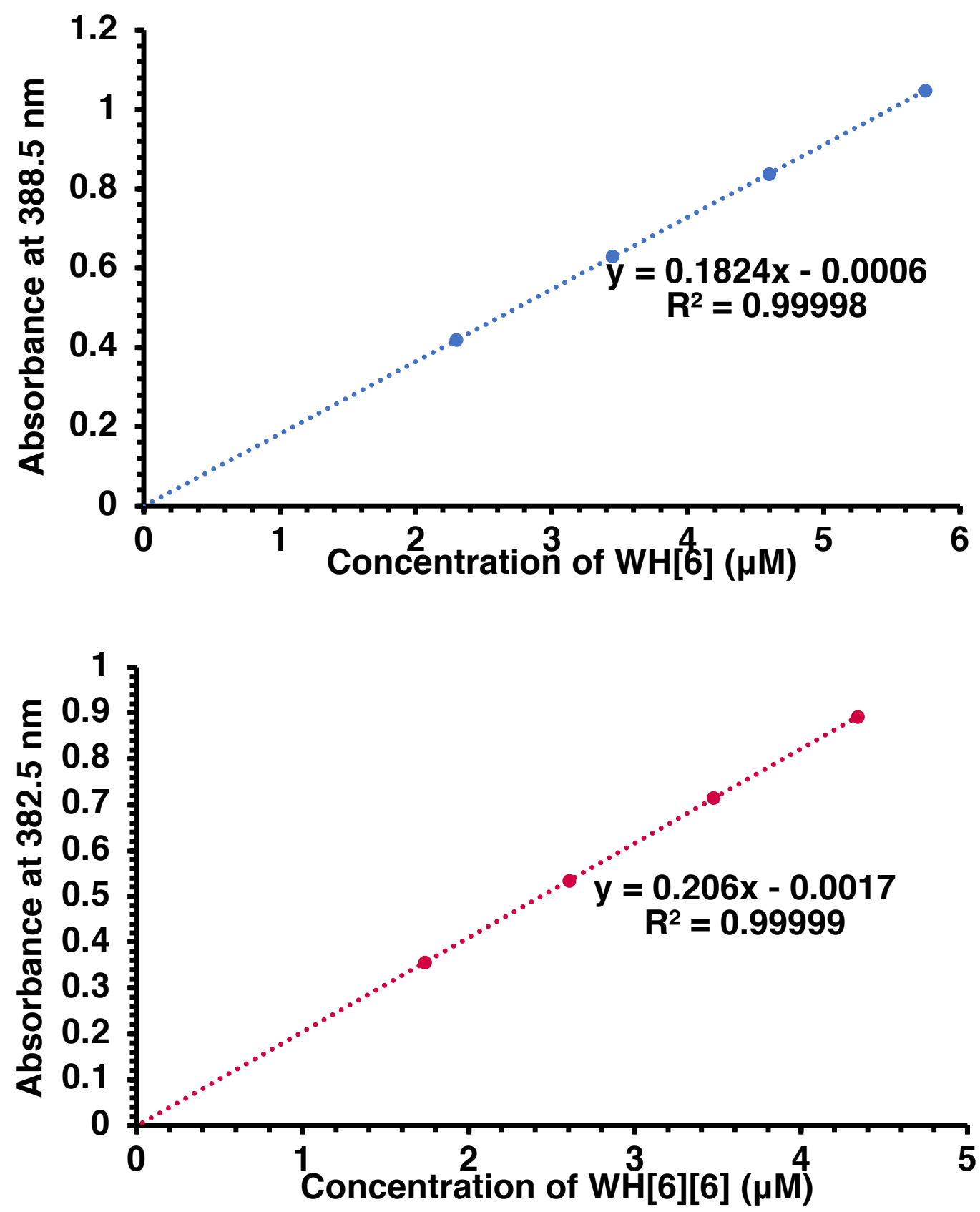

Figure S42. Beer-Lambert plots of WH[6] and WH[6][6] in dichloromethane (10-6 $\mathrm{M}, 1 \mathrm{~cm}$ path length) at room temperature. 
The crystallographic data corresponding to racemic WH[6] have been deposited with the Cambridge Crystallographic Data Centre (CCDC 1978284).

\begin{tabular}{|c|c|}
\hline Formula & $\mathrm{C}_{198} \mathrm{H}_{208} \mathrm{~N}_{8} \mathrm{O}_{16}$ \\
\hline MW & 2955.71 \\
\hline Space group & $\mathrm{P} 2{ }_{1} / \mathrm{c}$ \\
\hline$a(\AA)$ & $40.116(3)$ \\
\hline$b(\AA)$ & $20.2528(12)$ \\
\hline$c(\AA)$ & $23.0493(15)$ \\
\hline$a\left(^{\circ}\right)$ & 90 \\
\hline$\beta\left({ }^{\circ}\right)$ & $100.858(6)$ \\
\hline$V\left({ }^{\circ}\right)$ & 90 \\
\hline$V\left(\AA^{3}\right)$ & 18392(2) \\
\hline $\mathbf{Z}$ & 4 \\
\hline$\rho_{\text {calc }}\left(\mathrm{g} \mathrm{cm}^{-3}\right)$ & 1.067 \\
\hline $\mathbf{T}(\mathrm{K})$ & 100 \\
\hline$\lambda(\AA ̊)$ & 1.54184 \\
\hline $2 \theta_{\min }, 2 \theta_{\max }$ & 7,101 \\
\hline Nref & 76725 \\
\hline$R($ int), $R(\sigma)$ & $.1445, .1265$ \\
\hline$\mu\left(\mathrm{mm}^{-1}\right)$ & 0.526 \\
\hline Size $(\mathrm{mm})$ & $.13 \times .10 \times .03$ \\
\hline $\mathbf{T}_{\max } / \mathrm{T}_{\min }$ & 1.87 \\
\hline Data & 19236 \\
\hline Restraints & 4267 \\
\hline Parameters & 1999 \\
\hline$R_{1}($ obs $)$ & 0.1806 \\
\hline$w_{R_{2}}$ (all) & 0.5347 \\
\hline $\mathrm{S}$ & 1.327 \\
\hline Peak, hole (e- $\left.\AA^{-3}\right)$ & $0.41,-0.38$ \\
\hline
\end{tabular}


a)

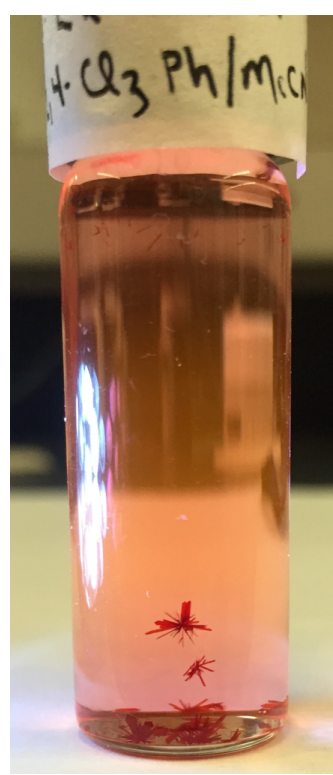

b)

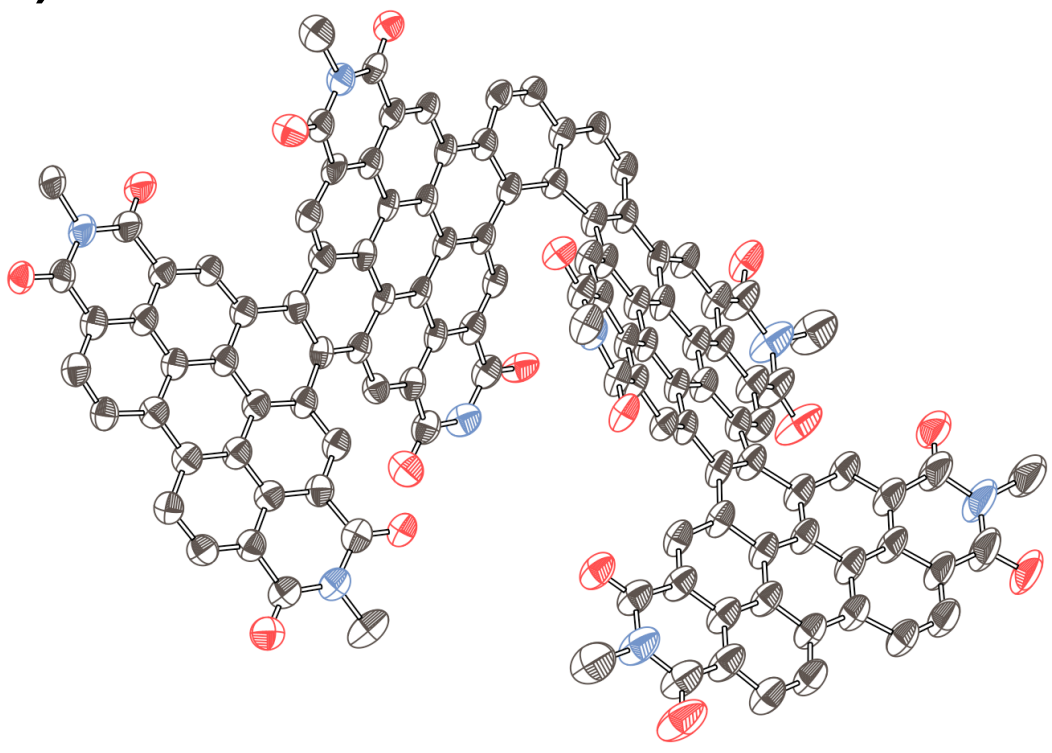

Figure S43. (a) Single crystals of WH[6] grown at room temperature from the vapor diffusion of acetonitrile into a solution of $\sim 1 \mathrm{mg}$ of racemic WH[6] in $\sim 2 \mathrm{~mL}$ of 1,2,4-trichlorobenzene. (b) Molecular structure of WH[6] as determined by SCXRD. The side chains have been truncated, and hydrogen atoms have been omitted for clarity. The thermal ellipsoids have been rendered at the $25 \%$ probability level.
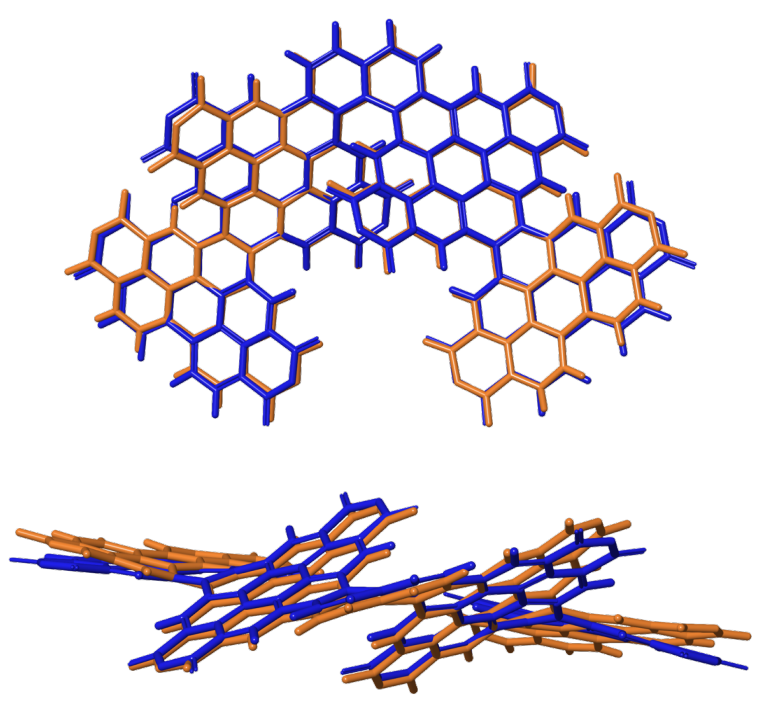
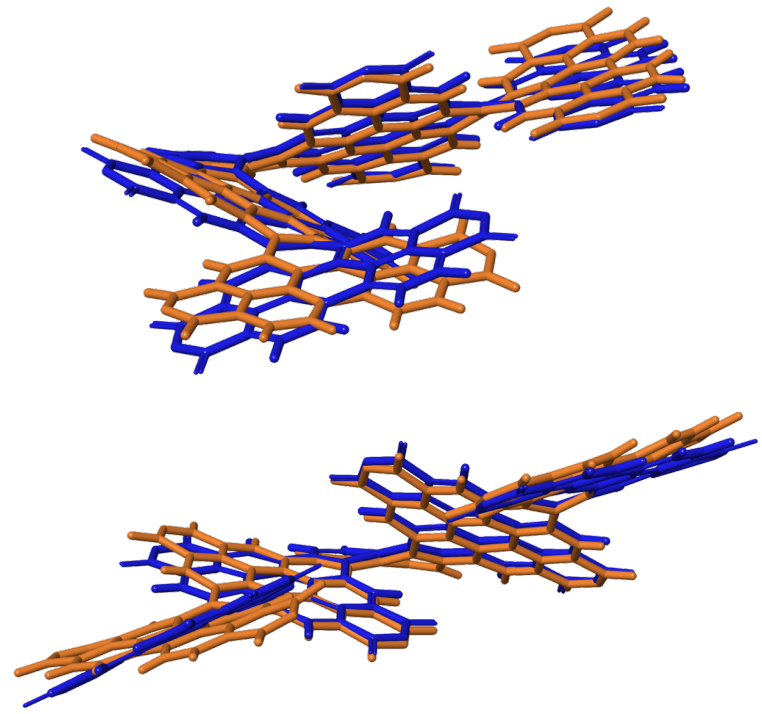

Figure S44. From different perspectives, the structure of PP-M[6]-PP from SCXRD (orange) superimposed on the DFT-optimized (B3LYP/6-31G**) structure of $\boldsymbol{P P}-\boldsymbol{M}$ [6]-PP (blue). The alkyl imide tails have been hidden to provide an unobstructed view of the $\pi$-surfaces. 


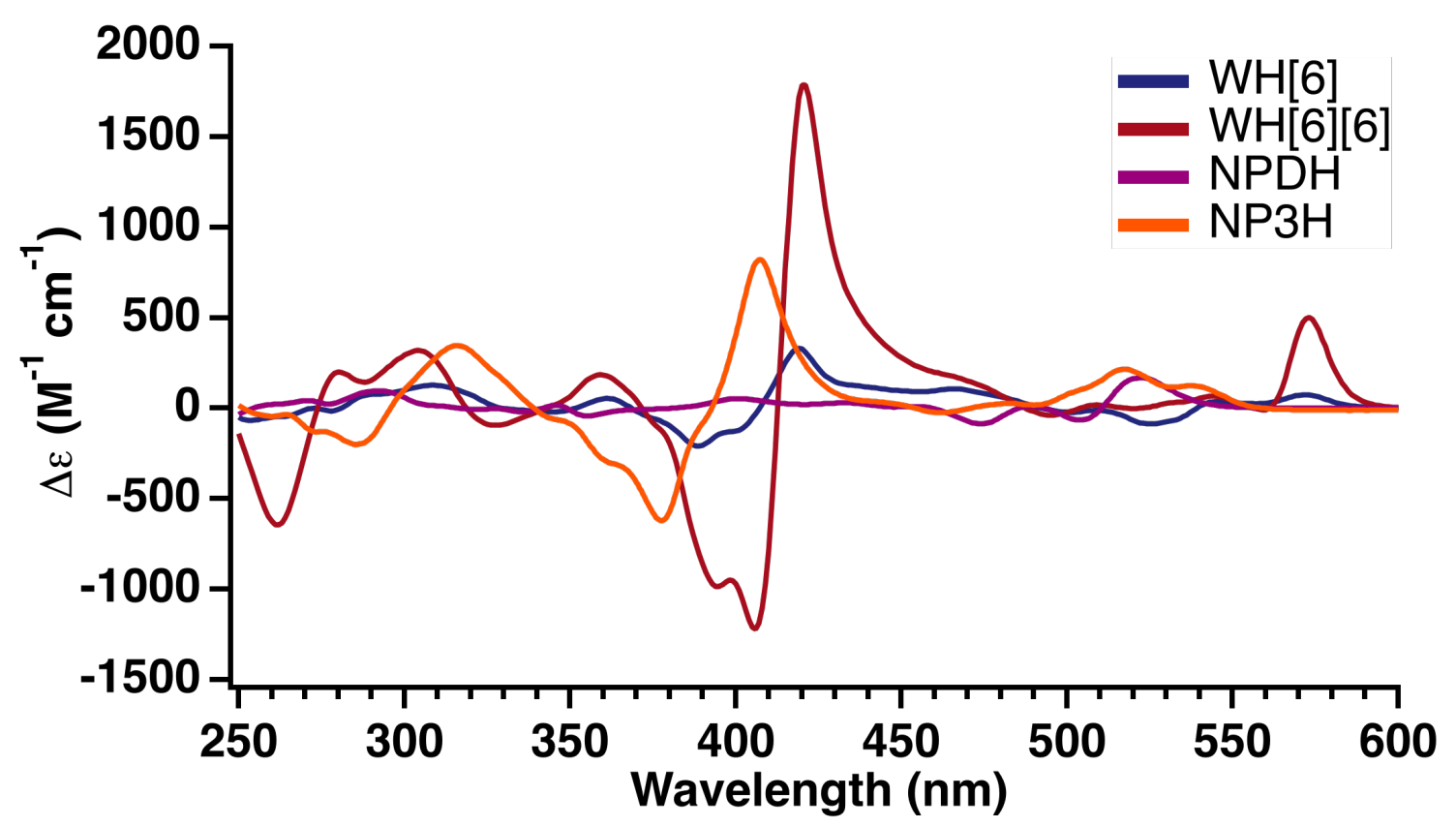

Figure S45. The ECD spectra of optically pure WH[6] and WH[6][6] in dichloromethane and NPDH and NP3H in tetrahydrofuran at room temperature. 


\section{References}

(1) Schuster, N. J.; Paley, D. W.; Jockusch, S.; Ng, F.; Steigerwald, M. L.; Nuckolls, C. Electron Delocalization in Perylene Diimide Helicenes. Angew. Chem. Int. Ed. 2016, 55, 13519-13523.

(2) Zhong, Y.; Kumar, B.; Oh, S.; Trinh, M. T.; Wu, Y.; Elbert, K.; Li, P.; Zhu, X.; Xiao, S.; Ng, F.; Steigerwald, M. L.; Nuckolls, C. Helical Ribbons for Molecular Electronics. J. Am. Chem. Soc. 2014, 136, 8122-8130.

(3) Khokhlov, K.; Schuster, N. J.; Ng, F.; Nuckolls, C. Functionalized Helical Building Blocks for Nanoelectronics. Org. Lett. 2018, 20, 1991-1994.

(4) Fulmer, G. R.; Miller, A. J. M.; Sherden, N. H.; Gottlieb, H. E.; Nudelman, A.; Stoltz, B. M.; Bercaw, J. E.; Goldberg, K. I. NMR Chemical Shifts of Trace Impurities: Common Laboratory Solvents, Organics, and Gases in Deuterated Solvents Relevant to the Organometallic Chemist. Organometallics 2010, 29, 2176-2179.

(5) Rajasingh, P.; Cohen, R.; Shirman, E.; Shimon, L. J. W.; Rybtchinski, B. Selective Bromination of Perylene Diimides under Mild Conditions. J. Org. Chem. 2007, 72, 5973-5979.

(6) CrysAlisPro 1.171.38.46. Rigaku Oxford Diffraction: Yarnton, England 2015.

(7) Sheldrick, G. M. SHELXT - Integrated Space-Group and Crystal-Structure Determination. Acta Crystallogr. Sect. A 2015, 71, 3-8.

(8) Sheldrick, G. M. Crystal Structure Refinement with SHELXL. Acta Crystallogr. Sect. C 2015, 71, 3-8.

(9) Dolomanov, O. V.; Bourhis, L. J.; Gildea, R. J.; Howard, J. A. K.; Puschmann, H. OLEX2: A Complete Structure Solution, Refinement and Analysis Program. J. Appl. Crystallogr. 2009, 42, 339-341.

(10) Spek, A. L. Structure Validation in Chemical Crystallography. Acta Crystallogr. Sect. D 2009, $65,148-155$.

(11) van der Sluis, P.; Spek, A. L. BYPASS: An Effective Method for the Refinement of Crystal Structures Containing Disordered Solvent Regions. Acta Crystallogr. Sect. A Found. Crystallogr. 1990, 46, 194-201.

(12) CrystalMaker Software Ltd.: Oxford, England (www.crystalmaker.com)

(13) Bochevarov, A. D.; Harder, E.; Hughes, T. F.; Greenwood, J. R.; Braden, D. A.; Philipp, D. M.; Rinaldo, D.; Halls, M. D.; Zhang, J.; Friesner, R. A. Jaguar: A High-Performance Quantum Chemistry Software Program with Strengths in Life and Materials Sciences. Int. J. Quantum Chem. 2013, 113, 2110-2142.

(14) Frisch, M. J.; Trucks, G. W.; Schlegel, H. B.; Scuseria, G. E.; Robb, M. A.; Cheeseman, J. R.; Scalmani, G.; Barone, V.; Petersson, G. A.; Nakatsuji, H.; et al. Gaussian. Gaussian, Inc.: Wallingford, CT 2016.

(15) Bruhn, T.; Schaumlöffel, A.; Hemberger, Y.; Bringmann, G. SpecDis: Quantifying the Comparison of Calculated and Experimental Electronic Circular Dichroism Spectra. Chirality 2013, 25, 243-249. 